From the collection of the

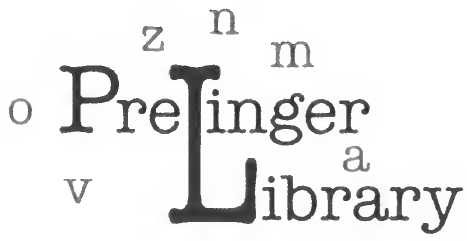

$\mathrm{t}$

$\mathrm{p}$

San Francisco, California 2006 


$$
9.95
$$




\section{Digitized by the Internet Archive in 2006 with funding from Microsoft Corporation}




\section{HUMAN GENETICS}

AND ITS SOCIAL IMPORT 


\title{
HUMAN GENETICS
}

\author{
AND ITS \\ SOCIAL IMPORT
}

\author{
BY \\ S. J. HOLMES \\ Professor of Zoology in the \\ University of California
}

First Edition

Third Impression

McGRAW-HILL BOOK COMPANY, INc.

NEW YORK AND LONDON

I 936 
Copyright, 1936, by the

McGraw-Hill Book Company, Inc.

PRINTED IN THE UNITED STATES OF AMERICA

All rights reserved. This book, or parts thereof, may not be reproduced in any form without permission of the publishers.

THE MAPLE PRESS COMPANY, YORK, PA. 


\section{Preface}

WHEN Herbert Spencer wrote his well-known book on The Study of Sociology he devoted a chapter to showing that sociology rests upon biological foundations and that preparation in biology is therefore necessary for the proper cultivation of the social sciences. The progress of biology has yielded abundant support for this conclusion and has shown that the connections between biology and sociology are more numerous and intimate than was probably appreciated even by Spencer. If one looks through a number of recent general treatises on sociology, it will be found that most of them devote considerable space to biological subjects, amounting in some cases to a quarter or a third of the contents of the book. In endeavoring to select those aspects of biology that will prove to be of the greatest service to students of social phenomena one is often perplexed by doubts as to whether one has made the best choice of material to present. Biology contributes to the social sciences in many ways. In fact, anything that helps us to understand man has its value for students of social relationships. Being compelled, there ore, to limit his field the author has confined himself mainly to the phenomena of human heredity and natural increase, and to some of the social consequences that are the products of these biological factors.

It is especially important that the student who attempts to arrive at correct judgments on controversial topics in eugenics and other fields of social biology should obtain some acquaintance with the common statistical and other fallacies that so often lead the unwary astray. Attention is called to a number of these, and I hope that the perusal of the present volume may do something toward developing a critical attitude of mind and an ability to form reasonable con- 
clusions, whether or not they finally prove to be right, on matters upon which opinions are now divided. We face many problems of social biology that urgently call for solution. What shall be done with the hereditarily defective classes? How shall we control immigration in the best interest of future generations? In what ways can we hope to overcome the evils of the differential birth rate? These and many other questions bring us face to face with issues upon which we find people stoutly maintaining opposed views. We cannot answer any of these questions without some knowledge of genetics. They are social problems, but they can be solved only by a study of biological facts.

It is desirable that readers of the present volume should have some acquaintance with the fundamentals of general biology, although very little technical knowledge is presupposed. Students should have access to some of the general treatises on genetics and they should be able to consult the more recent standard works dealing with problems of population. A few suggested readings in connection with the topics of the several chapters have been indicated, and a series of questions at the end of each chapter has been appended in the hope that they may prove helpful in giving the student who attempts to answer them a more adequate comprehension of the subjects treated.

The author is indebted to his colleague Dr. S. Light for reading the first nine chapters, and to his wife for her critical perusal of the entire manuscript. Dr. R. C. Cook has kindly permitted the reproduction of several figures from the Fournal of Heredity, of which he is the editor. Thanks are due to Dr. C. B. Davenport for the privilege of reproducing Figs. $3^{8}$ and 39 from Eugenical News.

S. J. Holmes.

Berkeley, Calif., April, 1936. 


\section{Contents}

PREFACE

CHAPTER I

Heredity and Its Cellular Basis . . . . . . . . . . i

CHAPTER II

Are Acquired Characters Inherited? . . . . . . . . . . ig

CHAPTER III

Mendel's Law. . . . . . . . . . . . . . . . . . . . . 29

CHAPTER IV

Heredity and Sex. . . . . . . . . . . . . . . . $4^{2}$

CHAPTER V

The Interaction of Factors. . . . . . . . . . . 52

CHAPTER VI

The Linkage of Hereditary Traits. . . . . . . . . . . 66

CHAPTER VII

Variability-Its Kinds and Its Causes. . . . . . . . 76

CHAPTER VIII

The Measurement of Variation . . . . . . . . . . . 87

CHAPTER IX

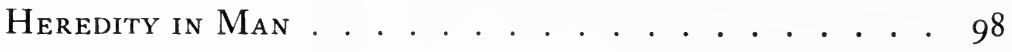

CHAPTER $\mathrm{X}$

Heredity and Environment . . . . . . . . . ili2

CHAPTER XI

Heredity in Mental Defect and Disease. . . . . . . 126

CHAPTER XII

Nature and Nurture in Mental Development . . . . I49 vii 
viii

\section{CONTENTS}

CHAPTER XIII

PAGE

Genetic Factors in Crime and Delinguency . . . . . i i I CHAPTER XIV

The Social-Problem People . . . . . . . . . . . . . I83

CHAPTER XV

Choice in Mating. . . . . . . . . . . . . . . I 88

CHAPTER XVI

The Birth Rate and the Causes of Its Decline. . . . . I96

CHAPTER XVII

The Differential Birth Rate .......... . 216

CHAPTER XVIII

Death Rates ................ . . 238

CHAPTER XIX

The Selective Action of Mortality . . . . . . . . 253

CHAPTER XX

The Biological Effects of War . . . . . . . . . . 274

CHAPTER XXI

The Effects of Population Growth . . . . . . . . . 290

CHAPTER XXII

The Growth of Cities and Its Demographic Effects . . 3ig

CHAPTER XXIII

The Biological Effects of Migration . . . . . . . 330

CHAPTER XXIV

Inbreeding ANd Crossbreeding. . . . . . . . . . . . . 349

CHAPTER XXV

Proposed Measures for Race Betterment . . . . . . . 359

Bibliography . . . . . . . . . . . . . . . . . . . . 387

INDEX. . . . . . . . . . . . . . . . 405 


\section{HUMAN GENETICS AND ITS SOCIAL IMPORT}

\section{CHAPTER I \\ HEREDITY AND ITS CELLULAR BASIS}

F ALL human beings had precisely the same hereditary 1 qualities, many of the social problems that confront us would wear a very different aspect. But the inherited traits of people are far from being exactly alike. It is a cardinal principle of human biology that, with the exception of identical twins, all men are born unequal, whatever may be said concerning their freedom or their inalienable rights. No one can doubt that the anthropological characters which distinguish Negroes, Nordics, Chinese, and Hindus profoundly affect the social, political, and economic relations of these peoples to other members of the human species. And within each race there are hereditary differences in physical form, facial contour, resistance to disease, intelligence, temperament, and countless other characteristics that influence the way in which individuals adjust themselves to their social environment. Hereditary differences, whether racial or individual, are therefore of interest to students of social problems as well as to the biologist. The more scientific knowledge we have concerning them the better able we shall be to deal with the problems they present.

The science of genetics, which is concerned with heredity and variation, has made enormously rapid progress during the past generation. Through its spectacular achievements it has attained more nearly the status of an exact science 
than any other branch of biology. One reason for this is that genetics, like physics and chemistry, has come to be mainly an experimental science. Previously most of what was known about heredity was gained by collecting instances of the transmission of some unusual trait. Writers on heredity accumulated many records of curious and interesting cases such as the perpetuation of extra fingers and toes, or the history of the family whose members could not endure the smell of cheese. Much was learned about heredity by simple observation, but, as in physics and chemistry, little progress was made in discovering the laws to which the phenomena conform until the subject was attacked by the method of controlled experimentation.

In addition to the insight gained through experimental breeding, our knowledge of genetics has been greatly enriched by the remarkable discoveries made by the cytologists regarding the cellular basis of hereditary transmission. Genetics and cytology have advanced hand in hand, inasmuch as the discoveries in the one field help us to interpret the phenomena observed in the other. In fact, some knowledge of cytology is now essential for a proper comprehension of genetics. Our first chapter, therefore, is devoted to the cell and some of the peculiar cellular processes associated with sexual reproduction.

One of the most fruitful generalizations of biology is the cell theory, according to which the bodies of higher plants and animals are made up of more or less individualized little units called cells. The first recorded observations on cells are credited to Robert Hooke, who described the cells of cork in his Micrographia published in I665. In the eighteenth century cells of various kinds were observed by Malpighi and by Grew in the tissues of plants and animals, but it was not until I838-I839 that the cell theory was formulated by the botanist Schleiden and the zoologist 
Schwann. This new doctrine of the make-up of organic bodies had a profound influence upon the development of morphology, physiology, and more recently genetics. According to this theory cells bear much the same relation to the body as do bricks to a house. The body thus interpreted is an aggregate of little units, each with a life and individuality of its own, but all cooperating in different ways to maintain the life of the whole. Cells are especially adapted to perform a variety of functions-muscle cells for contraction, nerve cells for conducting impulses, and gland cells for specific kinds of secretion. Each kind of cell has its particular part to play, just as individuals following different occupations play their parts in human society. For this reason, the expression "cell state" has often been applied to the organic body, and many analogies have been drawn between the social organism and the physiological organism. Whether with Herbert Spencer we speak of "society as an organism," or with Haeckel we designate the organism as a society or cell state, we express a fundamental likeness between a society and an individual organism in that each consists of more or less autonomous units whose activities are subordinated to a common end.

The original proponents of the cell theory held certain erroneous views as to how cells originate, but it soon came to be established that new cells are produced only by the division of other cells. The doctrine formulated by the pathologist Rudolf Virchow, Omnis cellula e cellula, soon came to be an integral part of the cell theory and exemplifies a further fundamental similarity between the social and the biological organism. The continued life of a society is brought about by the reproduction of its members, just as the reproduction of an individual organism is due to the multiplication of its component cells. Indeed, reproduction in both cases depends ultimately upon cell division. Embryonic 
development in the light of the cell theory is interpretable as the result of the division of cells, the growth of cells, the differentiation of cells, the arrangement of cells, and their mutual interactions-enormously complex series of processes, which, for reasons almost as mysterious as ever, are guided and controlled in such a way as to produce and maintain the beautifully coadapted aggregate of parts constituting the adult organism. Biologists look upon the cell as containing somehow the secrets of this almost miraculous phenomenon of embryonic development. All multicellular animals begin their embryonic development as a single cell, the ovum. To outward appearances, the ovum of a human being differs very little from an ovum that will give rise to a sheep, a dog, or a rabbit. Nor does it differ very conspicuously from an ovum that will develop into a jellyfish or even a plant.

The enormous potential differences inherent in these seemingly simple cells have led many biologists to postulate that the egg must possess a highly complex organization. There must be as many kinds of eggs as there are kinds of organisms. The eggs need not be so complex as the organisms to which they give rise, nevertheless there is no escape from the conclusion that every hereditary trait by which one species differs from another must have as its basis some differential peculiarity of the ovum. The same statement applies even to the individual hereditary differences within the same species. The ova giving rise to persons who inherit color blindness, six fingers, or the absence of incisor teeth differ from the ova that produce normal individuals by at least one factor corresponding to each of these peculiarities.

Since the secret of the mechanism of heredity is contained somehow in the make-up of the cell, let us consider some of the chief features of cell structure. A typical cell consists of a small bit of living protoplasm, or cytoplasm, containing 
a nucleus and surrounded by a cell wall. The latter structure, which was the first part of the cell to attract the attention of the older observers and which is responsible for the application of the term cell to those units of organic structure, is occasionally absent, as, for instance, in the white corpuscles of the blood. The cytoplasm, which usually constitutes the greater part of the cell, consists typically of a more or less jellylike protoplasm. This substance, which was designated by Huxley as "the physical basis of life," is a colloidal substance consisting of exceedingly

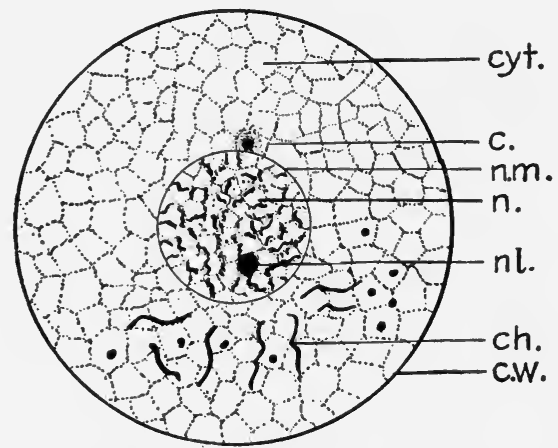

Fig. I.-Diagram of a typical cell. $c$, centrosome; $c h$, chondriosomes; $c y t$, cytoplasm; $c w$, cell wall; $n$, nucleus; $n l$ nucleolus; $n m$, nuclear membrane.

complex proteins and containing water, salts, carbohydrates, fats, and various products of its own metabolic activity. The nature of these contents and the peculiar structure of protoplasm itself vary according to the type of cell. In a cell of voluntary muscle, the cytoplasm has a highly differentiated fibrillar structure specialized in relation to contraction. The cytoplasm of a gland cell, or an epithelial cell of the skin, presents a very different appearance. There are many types of cells whose cytoplasmic structure varies in accordance with their diverse functions. In the ovum the cytoplasm commonly contains spheres or granules of yolk which serve as food material for the developing embryo. 
Some cells contain very large amounts of this substance; in birds, for instance, the so called yolk of the egg consists of one enormously enlarged cell.

The nucleus of a cell usually has much the same structure however varied the character of the cytoplasm by which it is surrounded. It is generally spherical in form, and its contents, which are enclosed by a thin nuclear membrane, present the appearance of a network in whose meshes lie numerous granules of a material called chromatin. This chromatin has a marked affinity for certain dyes, which are used for staining nuclei, so that they may be sharply distinguished from other parts of the cell. At times the
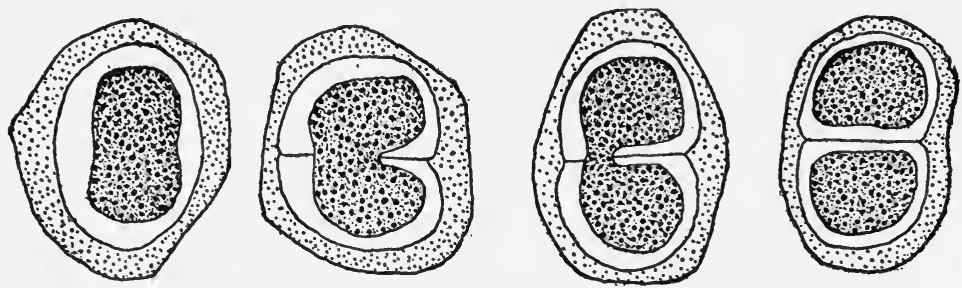

FIG. 2.-Amitosis, or direct cell division in ovarian nurse cells of the potato beetle, Leptinotarsa. (From Wieman.)

chromatin of the nucleus is aggregated into definite bodies, usually rod-like or thread-like in form, called chromosomes. As a rule chromosomes appear only during cell division, after which they again assume the appearance of a network.

The older students of the cell looked upon cell division as a very simple process. Both the nucleus and the cell body were supposed to become pinched in two forming two daughter cells. In some cases, cells do divide in this way (amitosis), but this is a very exceptional procedure and usually occurs only in cells that are more or less pathological and destined to undergo few subsequent divisions. The regular and typical mode of cell division is accomplished by a remarkable process, called mitosis, or karyokinesis. Commonly mitosis is inaugurated, by the division of a small 
body, the centrosome, lying in the cytoplasm close to the nucleus. The two daughter centrosomes gradually move apart, and around each appear numerous rays, giving rise
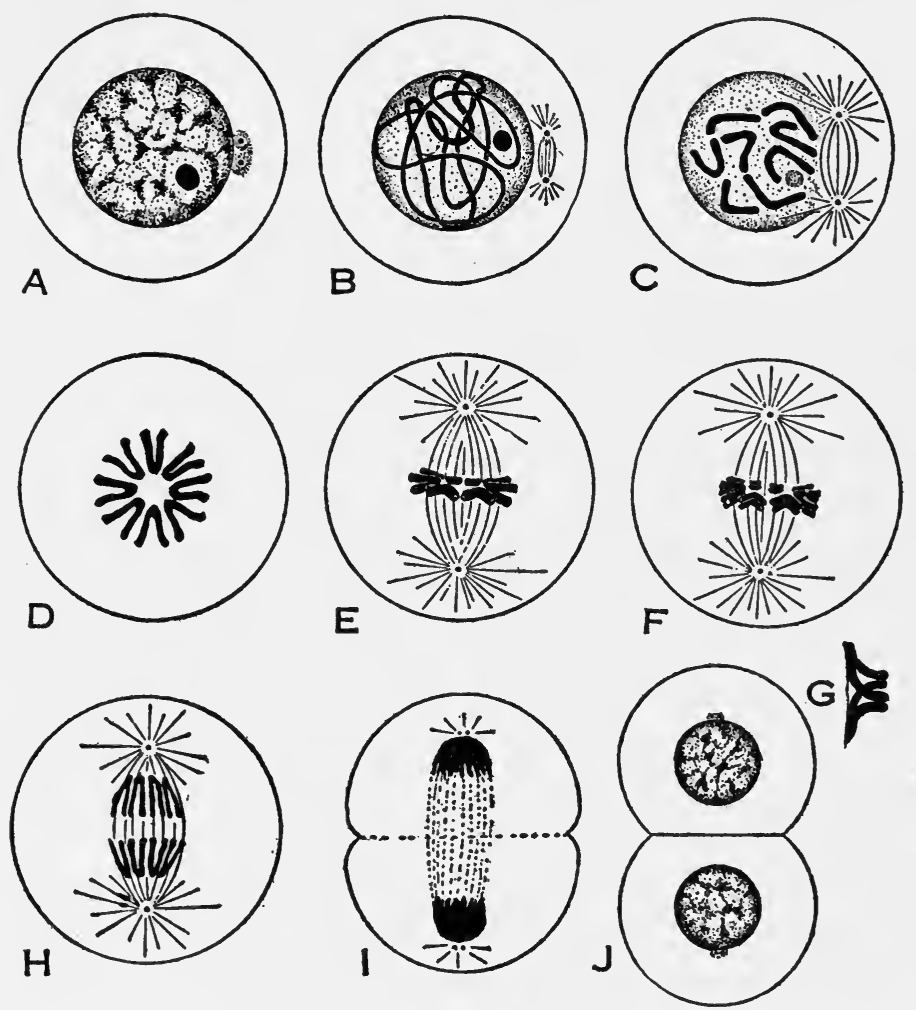

Fig 3.-Karyokinesis, or mitotic cell division. $A$, resting stage showing two centrosomes near the nucleus; $B$, beginning stage showing spindle and coiled thread of chromatin; $C$, later stage with larger spindle and eight chromosomes; $D$, polar view of chromosomes at the middle of the spindle; $E$, side view of spindle; $F$, a slightly later stage in which each chromosome is divided lengthwise; $G$, dividing chromosomes on spindle; $H$, the chromosomes pulled apart; $I$, end stage showing division of the cytoplasm; $\mathcal{F}$, completed division into two daughter cells. (From Wieman.)

to a stellate figure called the aster. Between the asters there develops a spindle-shaped body that appears to be traversed by a number of fibers. As the centrosomes move farther apart, the astral rays extend farther into the cytoplasm and the spindle enlarges. The nuclear membrane 
disappears, and the chromosomes, which in the meantime had been forming from the nuclear network, become drawn upon the spindle, taking up a position around the central part of it. Each chromosome as it lies on the equatorial region of the spindle appears to be divided lengthwise, the division being of ten manifest as a sort of double thread, long before the chromosomes take up their position in the mitotic figure. The chromosomes which seem to be attached to fibers extending from the centrosomes are then drawn apart. As the chromosomes approach the two poles of the spindle, they lose their regularity of outline and, after becoming surrounded by a new nuclear membrane, are finally incorporated into the two daughter nuclei. In the meantime the cytoplasm divides into two parts, thus completing the division of the cell. The spindle fibers and asters, which seem to have been called into existence to afford a mechanism for this peculiar kind of division, completely disappear after their work is done. Only the centrosomes may remain (and not even these in all cases) to form the starting point of the next ensuing division.

In this elaborate process each of the chromosomes is divided longitudinally into two equal parts. As was pointed out by Roux, the whole process of mitosis seems to be especially evolved for the achievement of this end. We now know that the precisely equal division of each and every chromosome is a matter of great importance in relation to heredity. There has accumulated a vast amount of critically tested evidence to the effect that chromosomes contain the factors which give rise to hereditary characters. Usually the number of chromosomes is constant in a given species, although it may vary between different species even of the same genus. In man the chromosome number is forty-eight, in the common fruit fly Drosophila melanogaster it is eight, and in the round worm Ascaris megalocephala it is four, 
and in one variety, univalens, only two. The chromosome number stands in no definite relation to position in the scale of life. In some of the one-celled Radiolaria the chromosome number is said to be over one thousand.

At one time there was a good deal of controversy over the so-called individuality of the chromosomes. Although the number of chromosomes is normally constant for a given species, it might be, and in fact it was, maintained that this constancy does not involve the persistence of each chromosome as an individual unit through the resting stages of the nucleus. Many characters of organisms, it was urged,

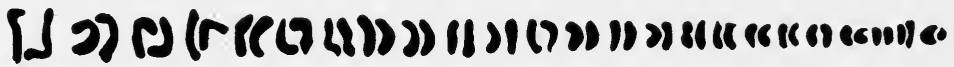

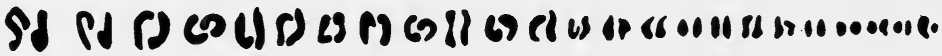

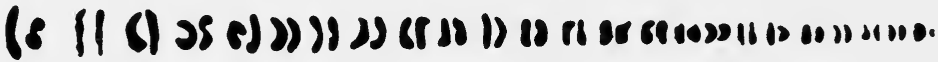

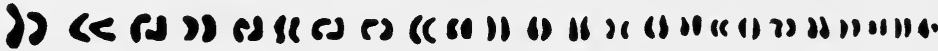

FIG. 4.-Chromosomes of man showing pairs of chromosomes from four spermatogonia. (After Evans and Swezy.)

are reproduced in numbers which are almost always the same. In common with nearly all mammals we have seven vertebrae in our necks, and a definite number of ribs, fingers, toes, and teeth, but no one supposes that these structures are represented by so many preformed parts in the germ plasm. The constant number as well as the structure of all these parts is the result of developmental processes. Organs are reproduced, not simply handed on. Hence it was inferred by some biologists that the constancy of chromosome numbers could be interpreted in the same way.

Usually no traces of individual chromosomes can be discerned in the resting nucleus of the cell. In this stage the chromosomes appear to be merged into the general nuclear network in which the visible chromatin is in the 
form of threads, granules, or irregular masses of larger size. When the chromosomes again take shape.in preparation for the next cell division it may well be doubted if each chromosome is composed of the same material as it was before it entered the resting stage. But despite many biological analogies that might be cited to the contrary, there is now very convincing evidence that chromosomes preserve their identity through successive cell divisions. Not only are chromosomes constant in number, but in many organisms individual chromosomes may be seen to retain their peculiarities of size and shape. This fact affords additional presumptive evidence for continuity, but it is not necessarily conclusive since it might be explained as due to the same sort of causes that lead to the repetition of similar shapes and sizes in the bones of our fingers and toes. If we could try the experiment of removing one or more chromosomes or possibly adding one or more extra ones and then see if the abnormal number was retained, we might obtain a crucial answer to our problem. Cytologists are able to perform marvelously delicate operations in dissecting the living cell, but the particular operation I have mentioned has not yet been accomplished. However, it is not really necessary to perform this experiment because Nature occasionally does it for us. Sometimes the chromosome number is doubled or even increased still more, or again it may be halved, and the changed number of chromosomes is found to persist through the resting stages of the nucleus. In other cases one or more chromosomes are lost, or there may be one or more extra chromosomes, and in these cases also the abnormal number is retained. Occasionally only a part of a particular chromosome is lost and this mutilated condition may be recognized through successive cell divisions. There are also other mishaps which befall the chromosomes at times, such as a piece of 
one chromosome being broken off and joined to another one in various positions, sometimes at one end or sometimes near the middle. Even these anomalies persist through many cell divisions and from generation to generation, and constitute permanent modifications of the hereditary peculiarities of the group. From such facts as these it is now practically certain that chromosomes retain their individuality through the resting stages of the nucleus. Just as each cell comes from a preexisting cell, so does each chromosome come from a preexisting chromosome. But what happens to the chromosomes when they can no longer be distinguished in the resting nucleus?

Apparently each chromosome must constitute a particular region of the resting nucleus, a sort of compartment of its own, so that when a chromosome emerges it is constituted of the same material as before. In the fish Fundulus and in some other forms the chromosomes as they separate in mitosis swell up into separate vesicles which remain more or less separate in the resting stages of the nucleus. In fact, each chromosome may be said to form a little nucleus of its own. Where the vesicles are closely packed together, the outline of each may be so obscured that the individual vesicles can no longer be distinguished. Probably something like this occurs in the usual type of cell division.

The persistence of chromosomes as individual units has a very important bearing on our interpretation of the physical mechanism of hereditary transmission. The chromosomes behave like so many little organisms multiplying by fission. In many species, as we shall see later, the chromosomes may be seen to be composed of still smaller units called chromomeres arranged in a linear series. Sometimes the chromosomes resemble strings of beads, as in those of the onion shown in Fig. 5. Still more remarkable details of chromosome structure have recently been discovered in the 
salivary glands of the larvae of the fruit fly, Drosophila. For some peculiar reason the chromosomes in these gland cells have assumed a perfectly enormous size, being about I 50 times as large as they are in ordinary cells. In stained preparations they show a very definite banded structure. Some of the bands are fairly broad and clear, others are faint, but hundreds of individual bands have been identified, and their position is normally constant in all the chromo-

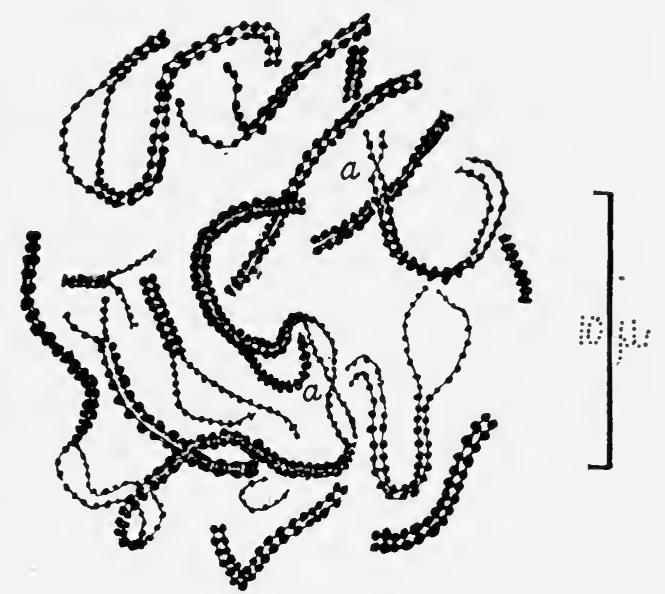

Fig. 5.-Chromosomes of the onion Allium triquetrum. The chromosomes appear to be made of strings of chromomeres which show a paired arrangement. (After Belling.)

somes of a given pair. An illustration of the so-called $X$-chromosome, or sex chromosome, is shown in Fig. 6. It is now possible, as is explained in a later chapter, to associate definite hereditary factors, or genes, with regions of individual chromosomes marked by these bands. Thanks to the occurrence of these giant chromosomes it is demonstrable that the little rod-like bodies that appear during mitotic cell division have a high degree of regional differentiation. The chromosomes of the fruit fly have now been mapped out in a degree of detail that may seem incredible to those who have not been initiated in to the mysteries of modern genetics. 
One can, for instance, locate the position of the genetic factor which causes the eye of the fruit fly to be white instead of red in a particular region very close to a band near the left end of the $X$-chromosome. Many other hereditary factors have been located with an equal degree of

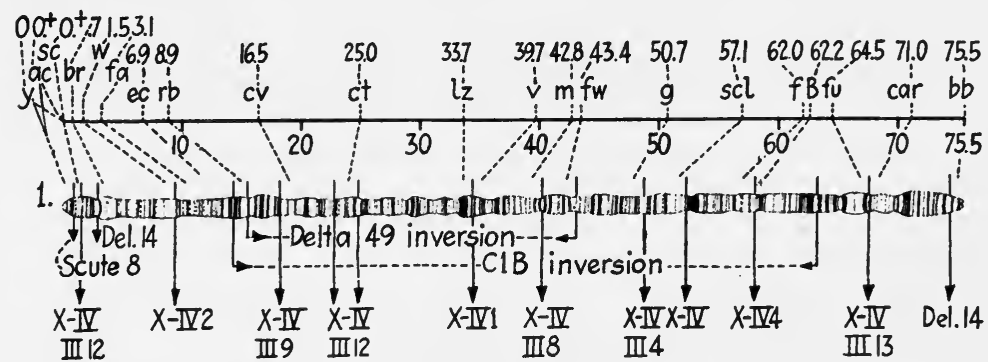

FIg. 6.-Giant $X$-chromosome from the salivary gland of the fruit fly Drosophila. For an explanation of the abbreviations see the map of the $X$-chromosome on p. 73. (After Painter.)

precision in the same or some other chromosome, and we may conclude, therefore, that each component part of a chromosome reproduces itself with great fidelity, and that the reproduction of chromosome parts is an essential element
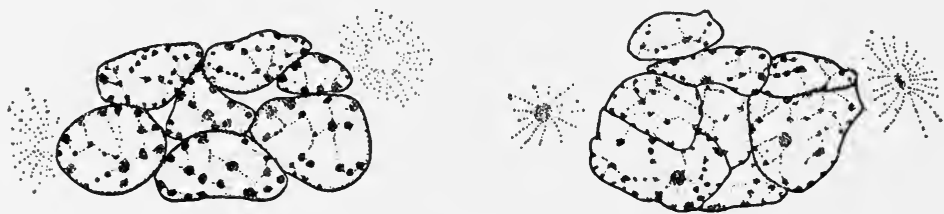

Fig. 7.-Chromosome vesicles of the fish Fundulus. Each chromosome swells up to form a separate vesicle which persists through the resting stages of the nucleus. Ordinarily the outlines of individual chromosomes can not be discerned in the resting stages, possibly because the vesicles are so closely pressed together. Compare the right-hand figure. (After Richards.)

in the transmission of hereditary traits. Some idea of the method by which these remarkable conclusions have been established will be given in a later chapter.

Although the chromosome number of a given species is maintained through countless series of cell divisions, there 
is one point in the life cycle in which there is an exception to this rule. In the animal kingdom this occurs during the formation of the sex cells, or gametes. During sexual reproduction, the egg cell is fertilized by a sperm cell from the sex gland of a male. In this process the nuclei of the male and female gametes unite to form the nucleus of the fertilized ovum. If the egg and sperm cells contain the number of chromosomes characteristic of the species, the number in the fertilized egg would be doubled in each

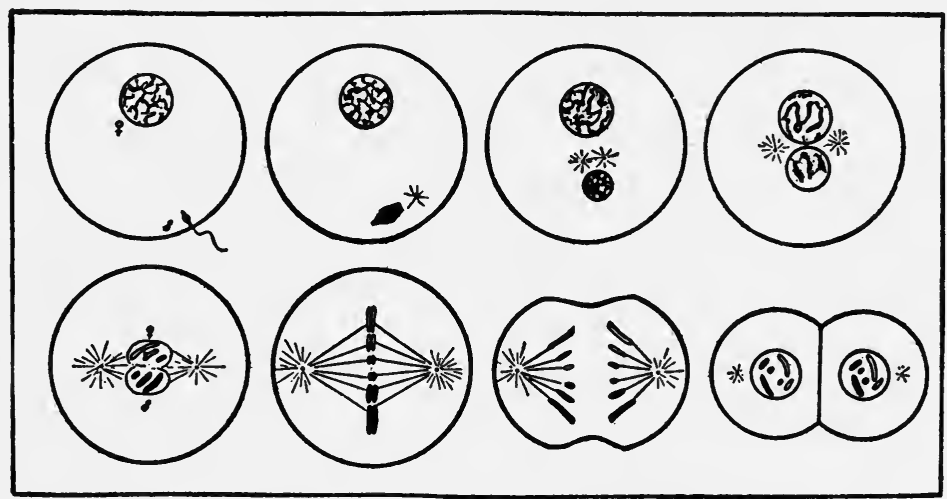

FIG. 8.-Diagram of the fertilization and first cleavage of the egg, showing the entrance of the sperm, the transformation of its head into the male pronucleus, the enlargement of the latter and its approach to the egg nucleus, the union of the egg and sperm nuclei and the division of the maternal and paternal chromosomes in mitosis. (After Sharp.)

generation. This consideration led Professor Weismann to postulate that prior to fertilization the germinal elements responsible for heredity must be reduced to one-half their previous number. This prophetic declaration received a striking confirmation through the study of the behavior of chromosomes during the final stages in the formation of the germ cells. In both oogenesis and spermatogenesis, it was found that, during the last two divisions, the chromosomes were reduced to half their previous number. The act of fertilization restores the number characteristic of the species. 
One may well ask why the sex cells should go through these peculiar maturation divisions and reduce their chromosome number to one-half if the original number is to be soon restored again in the act of fertilization? The answer

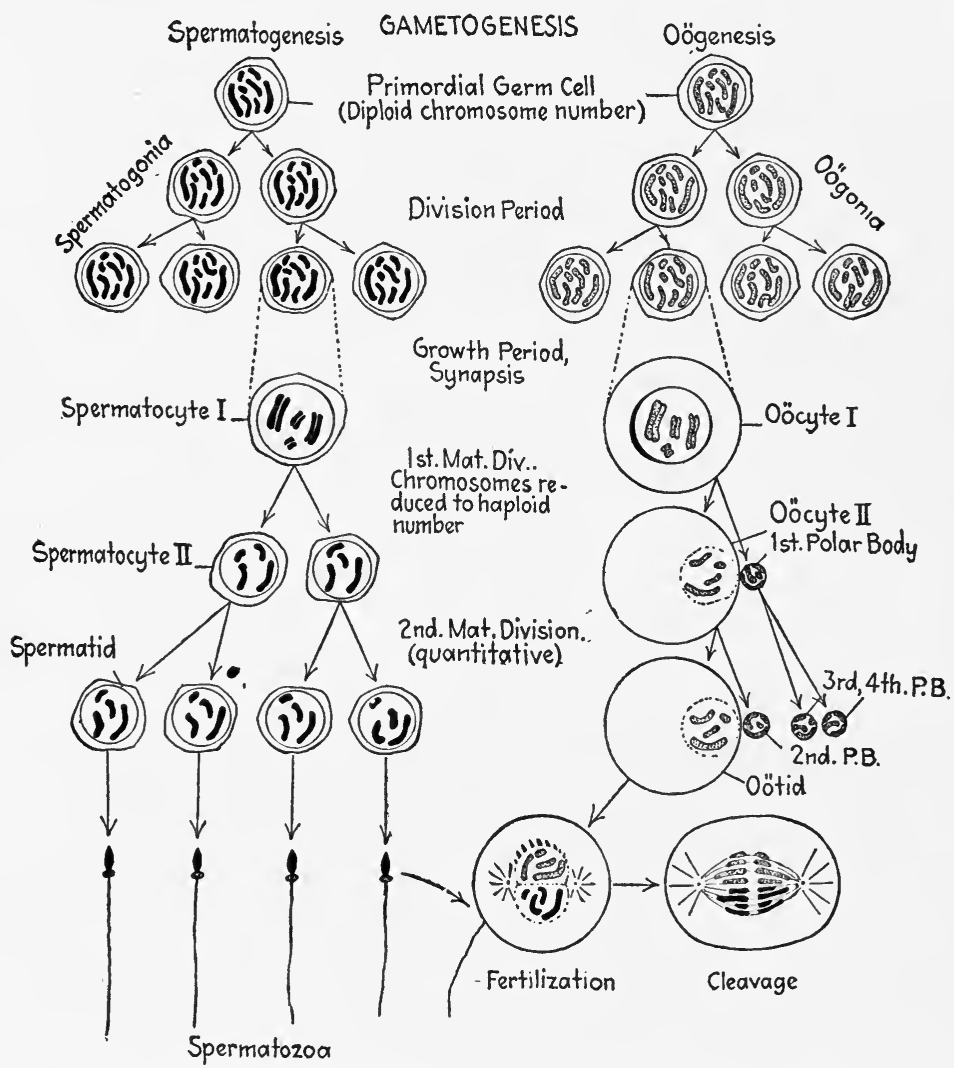

FIG. 9. - The formation of male and female sex cells (gametogenesis). Note the selective pairing of chromosomes in synapsis in spermatocyte $I$ and oöcyte $I$ and the subsequent reduction from eight to four in the maturation divisions. (From Wieman.)

to this question will give the clue to the biological significance of sex. It is evident that, although reduction and fertilization do not change the chromosome number, they effect a new combination of chromosomes, in that the chromosomes of the resulting individual are one-half of maternal and one-half 
of paternal origin. In other words, these processes provide the possibility of biparental inheritance.

Many years after the essential character of maturation and fertilization was established, another important feature of chromosome behavior was discovered, which proved to be of the highest significance in relation to the mechanism of heredity. This is the process of synapsis, or the pairing of the chromosomes, which takes place previous to the maturation divisions. When the chromosomes come together in pairs they do not fuse completely, but are intimately

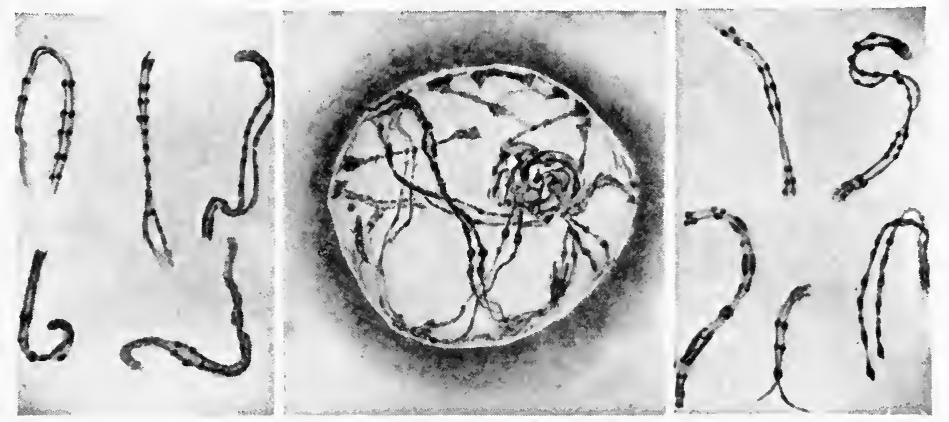

FIG. 10.-Pairs of chromosomes in the synapsis of Gasteria. Note the pairing of corresponding chromomeres. (After W.R. Taylor.)

associated for a time, after which they are separated in the maturation divisions and go in to different germ cells. There is convincing evidence that this pairing does not take place at random, but that each chromosome derived from the female parent pairs with a corresponding, or homologous chromosome derived from the male parent. Chromosomes are thus very discriminating as to the other chromosomes with which they pair. Each chromosome, drawn by what subtle affinity we do not know, somehow finds its predestined mate. This highly selective pairing insures that, after the pairs separate, each germ cell gets a complete set of all the different kinds of chromosomes in the body. We now know that it is highly important that these sets be retained intact. 
In any one germ cell, the proportions of chromosomes of maternal or of paternal origin may vary - this is a matter of chance-but each germ cell has one representative of all the different kinds of chromosomes characteristic of the species. After fertilization, each individual has two sets. Hence, sexually reproduced individuals are duplex in their inheritance.

It is natural to correlate the equality of chromosome contributions from the two parents with the fact that both parents contribute about equally to the hereditary qualities of their offspring. Here is one of the many facts supporting the so-called chromosome theory of heredity. But there are many other facts which show that we must look to the chromosomes and the way they get distributed and combined in the processes of sexual reproduction for the reasons why hereditary traits are handed on as they are. Let us now turn to the consideration of the ways in which heredity works.

\section{Suggested Readings}

Numbers in parentheses refer to dates of references listed in the Bibliography.

Shull ('31), chaps. 3, 4. Newman ('32), chap. I4. Further information in Sharp ('34) and Wilson ('28).

\section{Questions}

I. Why is the experimental method superior to mere observation and description in the attempt to discover the causes of phenomena?

2. What method is most employed in the study of morphology, taxonomy, embryology, physiology, biochemistry, psychology, and the social sciences?

3. In what other respects besides those mentioned in the text does a society resemble an individual organism?

4. If amitotic cell division occurred during the development of the germ cells, how would it probably affect the subsequent chromosome mechanism?

5. Do organisms differ hereditarily in more characters than they have chromosomes? 


\section{I8 HUMAN GENETICS AND ITS SOCIAL IMPORT}

6. How many chromosomes are there in the mature egg cells and sperm cells of man, the common fruit fly, and Ascaris megalocephala, variety univalens?

7. If one species of mammal had forty-eight chromosomes and another forty-four chromosomes, how many chromosomes would you expect to find in the hybrid resulting from crossing the two species?

8. Suppose a species had a range of chromosomes from large to small. Would it be possible through breeding for some forms to contain all the large chromosomes and others to contain all the small ones?

9. Why does synapsis result in maintaining intact the chromosome sets of a species?

I0. What are the evidences for the individuality of the chromosomes?

Ir. In what respects are oogenesis and spermatogenesis alike and in what respects do they differ?

12. Make a diagram of the chromosomes of the fruit fly, the chromosomes of the egg and the sperm cells, and those of the fertilized egg. How are these chromosomes distributed to the cells that arise from the cleavage of the egg? 


\section{CHAPTER II}

\section{ARE ACQUIRED CHARACTERS INHERITED?}

GORMERLY heredity was looked upon as involving I somehow a transfer of qualities from the various parts of the parental body to corresponding parts of the bodies of the offspring. The red hair or snub nose of a child was thought to be derived in some way from the red hair or snub nose of its father or mother. How such a transfer of qualities could take place was not apparent, but there were various theories about it. In accordance with this concept it was natural to conclude that characters acquired by the parents can be handed on, at least in some degree, to subsequent generations. According to the great French naturalist Lamarck, the evolution of organic life is largely the result of the transmission of acquired characters. At present most geneticists regard the Lamarckian theory with much suspicion. Many people are strongly disposed to believe in the transmission of acquired characters, however, because it seems to offer greater possibilities for the progress of the race. They do not like to think that the culture and training which individuals acquire have no effect upon the inborn qualities of their offspring. There are of course two sides to this aspect of the question. If children do not profit by the education and experience of their parents, they escape being afflicted by the mutilations, deformities, and general decrepitude that fall to the lot of so large a proportion of humanity. Among human beings at least the transmission of acquired characters might prove to be more of a misfortune than a benefit. 
At one time biologists considered that acquired characters are about as likely to be transmitted as any others. In fact, no sharp distinction was made between characters that are due to heredity and those which are acquired as a result of experience with the environment. According to some of the earlier theories of heredity, such as Spencer's ingenious theory of physiological units and Darwin's theory

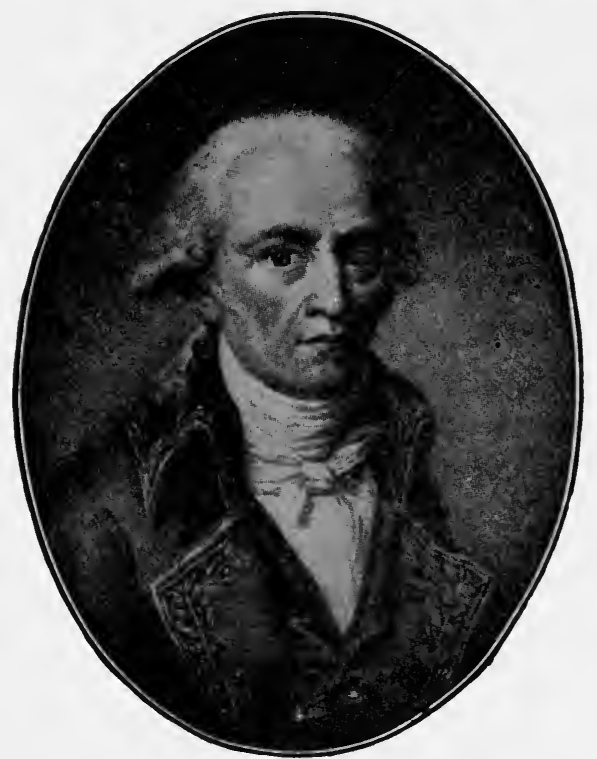

Fig. I I.-J. B. Lamarck. (After Locy.)

of pangenesis, both acquired characters and congenital characters are supposed to be handed on by essentially the same type of mechanism. In his great work on Variation of Animals and Plants under Domestication, Darwin accumulated an enormous fund of information concerning variation and hereditary transmission, and in the last chapter he set forth what he modestly called a provisional theory of heredity. He supposed that all parts of the body give off minute vital units called gemmules, which are capable of growth and multiplication. These gemmules were supposed 
to collect in the germ cells. During development, the gemmules derived from the various parts of the body were supposed to impress their several characteristics upon the corresponding parts of the offspring. If for any reason the distribution of gemmules was disturbed, a variation would result. If a part were increased through use, it would give off more gemmules, and the germ cells having more gemmules of this particular kind would produce offspring with the part more fully developed. Darwin's theory thus lent itself very readily to the explanation of the transmission of acquired

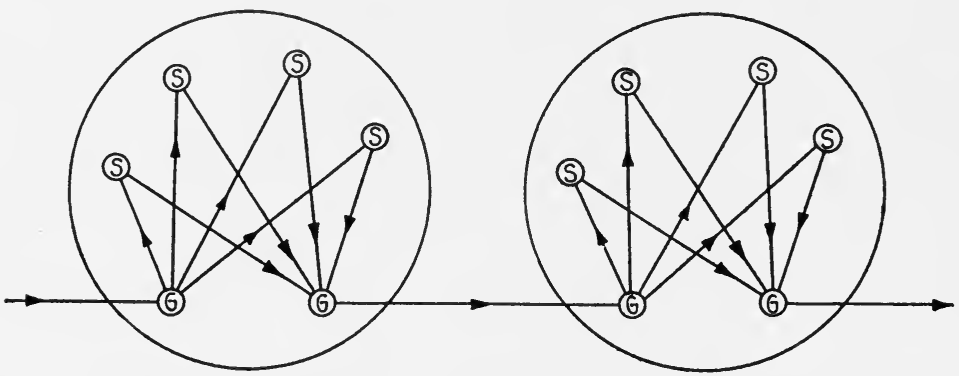

FIG. 12.-Diagram illustrating the course of the gemmules according to Darwin's theory of pangenesis. The gemmules from the parts of the body, $S, S$, etc., are supposed to collect in the germ cells, $G$, and to give rise to corresponding parts of the body in the offspring.

characters. Since Darwin believed in this doctrine, and sometimes appealed to it in his evolutionary speculations, he felt that an adequate theory of heredity must be able to explain the possibility of such transmission. His theory of pangenesis gave a concrete expression of the common conception of heredity to which we have alluded, as involving transmission from parts of the body of parents to corresponding parts of the body of their progeny. The process may be represented by the foregoing diagram (Fig. I2). Gemmules are of course purely hypothetical entities. Their postulated behavior was soon recognized as improbable from the standpoint of physiology. The theory of pangenesis, although it was worked out in a very ingenious way and subsumed 
many facts of heredity and variation under a common standpoint, never succeeded in securing much support and was finally completed abandoned.

Among other theories to explain the transmission of acquired characters are the various forms of the mnemic theory, according to which heredity is somehow akin to memory, and development is analogous to recollection. This doctrine first set forth by Hering has made a strong appeal to Semon, the late Samuel Butler, and also Mr. Bernard Shaw. But the theory is too vague and nebulous to appeal to most hard-headed geneticists. The Lamarckians have always found difficulty in presenting a plausible theory as to how acquired characters can be handed on. One is not justified, however, in asserting that a process cannot occur because there seems to be no reasonable explanation of how it could happen. We have by no means unraveled all the intricacies of life processes, and we cannot afford to be dogmatic about Lamarckian inheritance because it is not what we would expect in the light of our present knowledge of the mechanism of heredity. But we should be skeptical and not accept the theory unless adequate evidence can be produced in its support.

A potent factor in causing biologists to become distrustful of the Lamarckian theory was the attacks of Weismann during the eighties and nineties. Weismann had a theory of heredity of his own, based upon the concept of the continuity of the germ plasm. The germ plasm was supposed to be derived, not from the body or soma, but from the antecedent germ plasm of the parents, which was supposed to be passed on in an unbroken stream through countless generations. A part of the germ plasm becomes differentiated into the body cells, but a small portion of it persists unchanged in the germ cells. In Weismann's view the germ cells give rise to the body cells, but the germ cells are not 
strictly speaking derived from the body but from antecedent germ plasm. The reason why offspring resemble their parents is because both arise from a common substance, of which their bodies are the carriers. This is a very simple way of looking upon heredity. Moreover, it harmonizes very nicely with the cell theory. Weismann held that the part of the cell responsible for hereditary transmission is the nucleus and more particularly the chromosomes. And since chromosomes arise from the division of antecedent chromosomes, these bodies would seem to be admirably adapted to serve as carriers of hereditary factors.

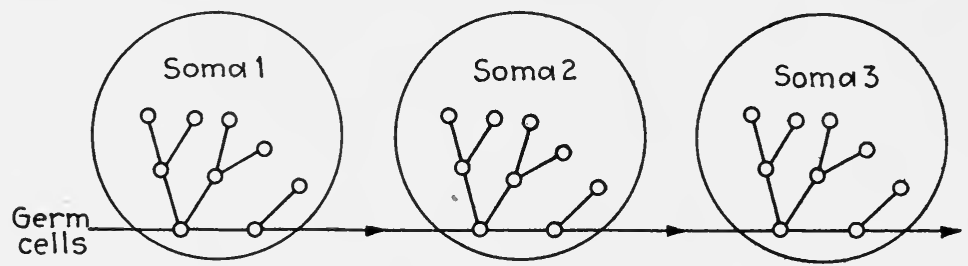

FIG. 13.-Diagram illustrating Weismann's conception of the continuity of the germ plasm. The germ cells produce other germ cells, and also the other cells that constitute the body, or soma.

The one feature that distinguishes Weismann's theory most sharply from the theory of Darwin is that hereditary qualities are not derived from the bodies of parents. Like plants arising from a common stolon or runner, successive generations are similar because of their common origin. If this theory is correct, it would seem to preclude the transmission of acquired characters, because there seems to be no way in which such characters can be passed into the germ plasm. According to this theory, therefore, one would not expect acquired characters to be inherited.

Having espoused his theory of the continuity of the germ plasm, Professor Weismann was very naturally led to examine the evidence for the transmission of acquired characters and came to the conclusion that it was far from conclusive. His criticisms convinced a large proportion of 
his biological colleagues that the Lamarckian theory had been accepted upon insufficient grounds. Many biologists, however, remained devoted Lamarckians, and while the theory has steadily lost adherents, there are still able biologists who stoutly defend it.

One of the first and most frequently mentioned tests of the Lamarckian theory was the experiment of cutting off the tails of mice to see if the progeny would have shorter tails. Although twenty-two generations of mice were subjected to this indignity their progeny continued to produce tails of the usual length. There have been reports of isolated cases where an accidental mutilation has been followed by a similar defect in the offspring. But there is no assurance that these cases may not be due merely to coincidence. Many savage tribes have the custom of gashing their cheeks, stretching their ears, filing their teeth, and flattening their skulls, but their children are born sound and normal, despite untold generations of such treatment. Biologists should feel grateful to their less enlightened brethren for these long-continued experiments on the hereditary effects of mutilations. There is no good evidence that the rite of circumcision has been productive of any inherited results. Even the Lamarckians concede that the evidence for the inherited affects of mutilations is mostly negative. They usually contend, however, that mutilation affords no real test of the Lamarckian theory, because if a part is removed, it is thereby rendered incapable of affecting the germ cells. But granting the relevancy of this contention, it may be pointed out that many kinds of mutilations, such as those shown in the figure, do not involve the loss of any part, but are merely changes in configuration, which, according to the theory, should produce some inherited effect.

The experiments on Lamarckian inheritance extending through the largest number of generations have been carried 
on with the common fruit fly, Drosophila. In order to test whether disuse of the wings would lead to their gradual reduction in a series of generations Lutz bred Drosophilas in small vials in which they were unable to fly. After fortynine generations, the average wing length was found to be fully as great as at the beginning of the experiment. A common explanation of rudimentary organs is that they result from the inherited effect of disuse. The theory seems
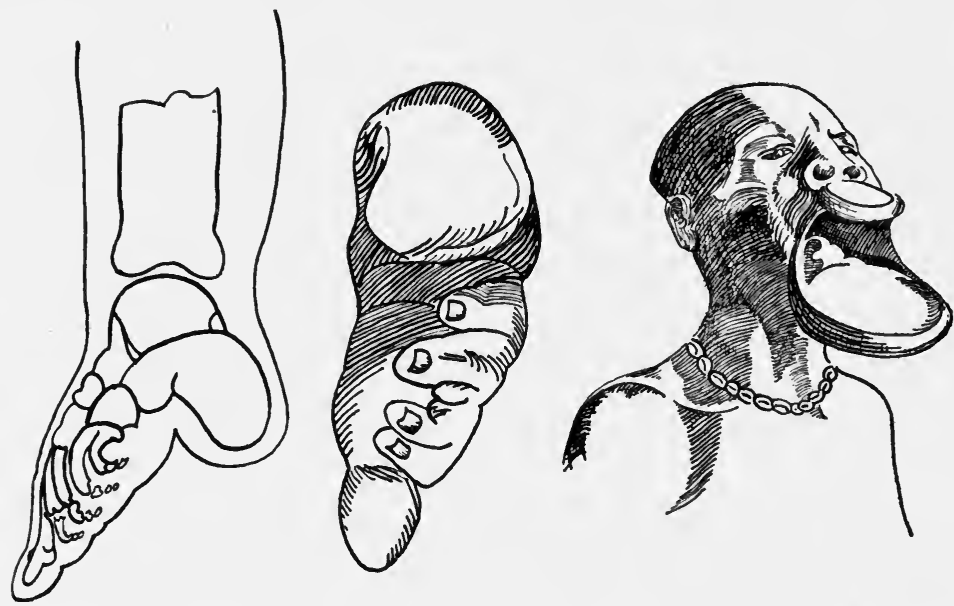

Fig. 14.-Mutilations practiced by different peoples. The two figures to the left represent the distorted foot of a Chinese lady. The right figure, after Haardt and Audouin-Dubreuil, shows an extreme distortion of the lips by means of flat disks. Generations of these and other deformities have failed to cause any noticeable effect upon the progeny.

plausible, but it lacks experimental proof. Dr. Payne bred fruit flies in the dark to see if their eyes would become reduced in size. Many cave animals which live in almost total darkness have either lost their eyes entirely or else possess eyes in various stages of atrophy. But Dr. Payne's fruit flies, even after sixty-nine generations in the dark, showed no reduction in the size of their eyes or in their ability to react to light. It is easy to say that some effect might be produced if the flies were kept in darkness for several thousand generations instead of sixty-nine. One 
must concede that this is possible, but possibilities of this sort do not constitute a satisfactory basis for accepting a theory.

While the results of these experiments are distinctly negative, other experiments have yielded results which have been claimed to support the Lamarckian doctrine. A number of these have been reported by the enthusiastic Lamarckian, Paul Kammerer, but the careless methods employed, the lack of adequate controls, and the rather sensational and declamatory manner in which the results were described have caused most biologists to look upon Kammerer's work with profound distrust. The veteran Russian physiologist Pavlov, justly famous for his classical investigations on the digestive glands and conditioned reflexes, described some experiments in which it was claimed that the progeny of mice, trained to come at the sound of a bell, learned to respond much more readily than the progeny of mice which were untrained. This startling announcement from so eminent a scientist was widely quoted. Similar experiments were repeated by other scientists who failed to confirm these results. And finally Pavlov, himself, stated that, owing to sources of error involved in the experiments, they could not be interpreted as yielding support to the Lamarckian doctrine.

The most thoroughgoing and carefully controlled experiments to test the inheritance of the effects of learning were carried out by Dr. W. McDougall upon rats that were trained to swim through the right passage of a maze. Several generations of rats were put through the experiment, and McDougall reported that the progeny of the trained rats were able to learn the maze more readily than those of rats which were untrained. The experiment was repeated by Crew who failed to obtain the same results, and who is disposed to explain the findings of McDougall on grounds 
other than the transmission of acquired characters. Others have criticised the experiments because the effects of selection were not adequately guarded against. Even McDougall did not claim that his results afforded a demonstration of Lamarckian inheritance, although they were held to yield strong support to this doctrine.

One might be disposed to think that the problem of the transmission of acquired character would be rather easy to settle, but the investigation of the subject is beset with many sources of error. Many experiments have been adduced in support of the Lamarckian theory, but they have all been criticised as inadequate. It would scarcely be profitable to discuss these experiments in detail. We shall have to be content with referring the student to the suggested readings on this subject at the end of chapter and the further list of references at the end of the volume.

\section{Suggested Readings}

Detlefson ('25), Guyer ('27), chaps. I5, I6. Newman ('32), chap. 3 I. Thompson ('13), chap. 7. See also Kammerer ('24), McDougall ('27), ('30), Weismann ('9r), ('93).

\section{Questions}

I. Is germ plasm necessarily different from body plasm? If not, is this fact opposed to the doctrine of its continuity?

2. What biological facts are plausibly explained by the Lamarckian theory?

3. The workers of the hive bee are normally sterile. What bearing does this fact have on the theory that their instincts have been gradually evolved through the transmission of acquired characters?

4. Discuss the Lamarckian theory in relation to protective coloration, the devices of seeds to facilitate dissemination, and the structures of flowers which favor cross-pollination through the visits of insects.

5. Is the transmission of immunity to disease from mother to offspring through the placenta an evidence of Lamarckian heredity?

6. What bearing does the doctrine Omnis chromosoma e chromosoma have on the probability of the transmission of acquired characters? 


\section{HUMAN GENETICS AND ITS SOCIAL IMPORT}

7. What are the results of transplanting ovaries from one animal to another, and how do they bear on the Lamarckian theory?

8. If alcoholic habits run in families, is it an evidence of Lamarckian heredity?

9. According to Darwin's theory of pangenesis would you expect that the removal of an organ would have a hereditary effect?

Io. If a child's parents both became deaf from scarlet fever, would you expect that the child's hearing would thereby become affected?

II. Is there any other explanation of the origin of rudimentary organs except that they result from the inherited effects of disuse? 


\section{CHAPTER III}

\section{MENDEL'S LAW}

$7 \mathrm{HE}$ discovery that has done more than anything else 1 to reduce the manifold phenomena of heredity to law and order was made by an Austrian monk Gregor Johann Mendel. By training Mendel was a physicist, but from his boyhood he had a strong interest in studying plants and animals. He had read Darwin's Origin of Species and the works of some of the older breeders of plants. Perhaps, he thought, some light on the species problem may be gained through a careful study of plant hybrids. For various reasons be chose for experimentation the common garden pea, Pisum sativum. There were several varieties of this plant available, differing in sharply defined characters, and Mendel focussed his attention on these particular features of the plant. In the garden of the monastery at Brünn, Austria, Mendel carried on for several years his experiments in crossing different varieties of garden peas. His principal results were embodied in a paper published in the Proceedings of the Natural History Society of Brünn in I866. This paper ranks as one of the finest achievements of experimental research. Nevertheless, no one paid any attention to it. There seems to be only one brief reference to it in the scientific literature before I900. Somewhat sadly Mendel was wont to remark, "Meine Zeit wird schon kommen"-My time will surely come. But it did not come until after he was dead. In 1900 through a remarkable coincidence Mendel's work was brought to light by three men, DeVries, Correns, and Tschermak, each of whom 
confirmed Mendel's results by independent investigations of his own. Then the science of genetics took on a new lease of life. It grew mightily. As a consequence more has been learned about heredity in the past thirty-five years than had been learned in all preceding time. Let us look at some of the results achieved by this patient and sagacious monk.

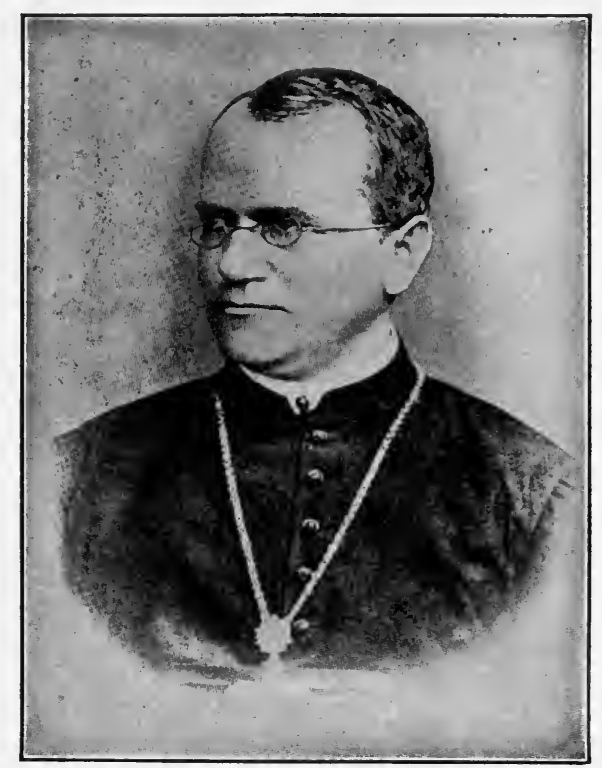

Fig. I5.-Gregor Johann Mendel. (From A. F. Shull.)

When Mendel crossed a tall with a dwarf pea, he found that the immediate progeny were all tall. Likewise when he crossed a yellow with a green pea, the progeny were all yellow. The characters tall and yellow were called dominant, while dwarf and green were called recessive. The appropriateness of the latter term is shown by the fact that in the second generation of hybrids, the recessive character reappears. And what is more remarkable it reappears in a definite numerical ratio, one-fourth of the progeny showing 
the recessive character, while three-fourths exhibit the dominant character. By a painstaking analysis Mendel demonstrated that the peas having the dominant trait were not all alike; two-thirds of them when self fertilized produced offspring which gave the same ratio of three dominants to one recessive, while one-third of them produced nothing but pure dominants. One may write a general formula for the second generation of hybrids as

$$
\text { I } D D+{ }_{2} D R+{ }_{\mathrm{I}} R R
$$

Both the pure or homozygous dominants, $D D$, and the pure recessives, $R R$, breed true indefinitely, while the mixed or heterozygous forms, $D R$, produce dominants and recessives in the 3:I ratio. This separation in the second generation of the characters that entered the cross is called the principle of segregation and represents the essence of Mendel's brilliant discovery. He found that all the seven pairs of characters with which he experimented in garden peas segregated out according to the $I: 2: \mathrm{I}$ ratio in the second generation.

A mind having Mendel's bent for accurate analysis was naturally led on to inquire what would happen if peas were crossed differing in two pairs of characters. In practice this proved a more difficult problem to solve, but in principle the solution was simple enough. The members of each pair were segregated out in the second generation quite independently of the members of the other pair. Thus, when a tall yellow pea was crossed with a dwarf green pea, the first, or $F_{1}$, generation were all tall yellow peas, but the second generation showed the following combinations:

9 tall yellow +3 tall green +3 dwarf yellow + I dwarf green

This is the ratio which would be obtained if the members of each pair were segregated independently of those of the 


\section{HUMAN GENETICS AND ITS SOCIAL IMPORT}

other. It illustrates what is called the "law of independent assortment." Mendel demonstrated that this principle applies to all the characters of peas with which he experimented. He also showed that if three pairs of characters are combined in a cross the same law obtains, but the ratios of the various combinations of characters are more involved.

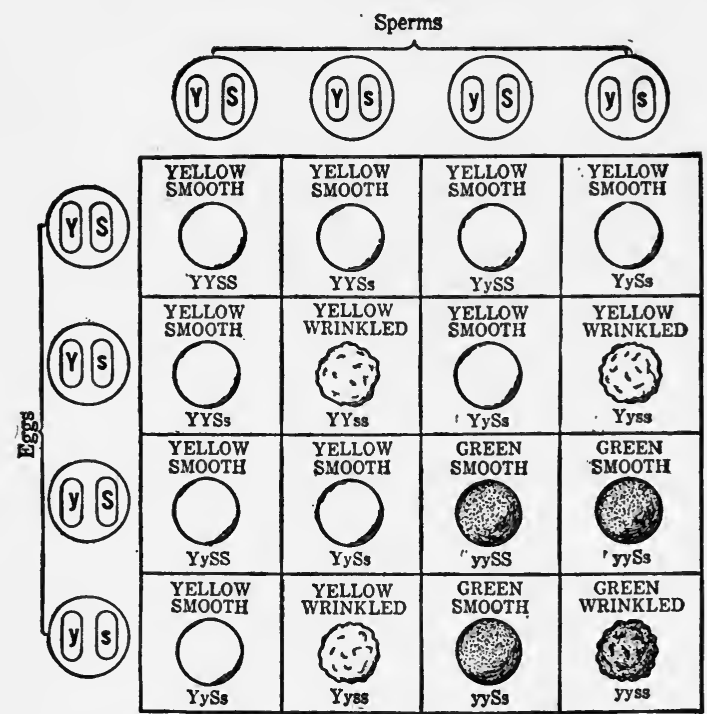

Fic. 16.-Diagram illustrating the dihybrid ratio obtained from crossing peas differing in two pairs of characters, a smooth yellow with a wrinkled green pea. Each of the four kinds of gametes of one individual can unite with any of the four kinds of gametes of the other individual and thus produce sixteen combinations shown in the squares. (From Haupt.)

If, for instance, we cross a tall, yellow, round seeded pea with a dwarf, green, wrinkled seeded pea, the second generation of the hybrid would be:

27 tall round yellow +9 tall round green +9 tall wrinkled yellow +9 dwarf round yellow +3 tall wrinkled green + 3 dwarf round green +3 dwarf wrinkled yellow + I dwarf wrinkled green

With four characters the ratios would be still more complicated. 
The demonstration that particular characters could be combined and segregated in perfectly definite mathematical ratios was a surprising revelation. Mathematical exactness is rarely encountered in the phenomena studied by biologists, and had Mendel been trained in the biological sciences instead of physics and chemistry, he might never have made his great discovery. According to Mendel's law, inheritance is essentially alternative so far as each unit character is concerned. Botanists and zoologists were familiar with inheritance of this type, but they regarded it as exceptional. Different characters in the parents were supposed to blend in the offspring and to produce a fairly permanent intermediate condition. There was no permanent blending in Mendel's peas. The progeny were round or wrinkled, tall or dwarf, and not something halfway between.

After Mendel's unnoticed papers were brought to light, biologists were spurred on to ascertain to what extent the principles that obtained in the crossing of garden peas might apply to plants and animals in general. Experiments on the breeding of all sorts of plants and animals were soon undertaken, and Mendel's law was found to hold true for a great variety of organisms in both the plant and animal kingdoms.

Quite naturally the question as to why the characters of plants and animals are inherited according to definite mathematical laws came prominently before the minds of all students of heredity. Mendel himself had speculated upon it, although nothing was known in his day of the cytological changes involved in the production of the germ cells. Nevertheless, he hit upon an interpretation which, so far as it goes, was shown later to be fundamentally correct. It was the doctrine of the purity of the gametes, or sex cells, for the factors upon which alternative inheritance depends. 
Mendel supposed that in the formation of the germ cells by the $F_{1}$ hybrid the factors for the two contrasted characters were separated so that each germ cell would contain one or the other factor, but never both or neither. Half of the germ cells would contain the dominant factor, and a half the recessive factor in a given pair of characters. If these germ cells meet at random in all possible combinations, they will produce offspring according to the ratio

$$
\mathrm{I} D D+2 D R+\mathrm{I} R R \text {. }
$$

Let us suppose that we have two bags containing equal numbers of red and green beads in each and I take a bead at random out of each bag. I might draw a green bead out of both, a red bead out of both, or a red bead out of the first and a green bead out of the second, or vice versa. In the long run my pairs of beads would consist approximately of $1 / 4$ reds, $1 / 4$ greens, and $1 / 2$ red and green. One may also obtain Mendelian ratios by tossing two pennies, which - if we toss them often enough-will turn up heads in $1 / 4$ the throws, tails in $1 / 4$, and a head and a tail in $1 / 2$ the throws. These ratios follow the laws of chance. Given enough cases, phenomena due to pure chance may conform to very definite statistical laws. Mendel showed that the segregation of hereditary characters obeys the laws of chance frequency distribution, and he rightly conjectured that the reason why they do this is because the contrasted unit factors in hybrid organisms come to be separated in the formation of the gametes, or sex cells. Since these cells with their different factors occur in equal numbers and are free to unite in all sorts of combinations, these combinations will on the average be formed in accordance with Mendel's law.

Apparently something must occur during the formation of the germ cells which is analogous to tossing pennies, 
and the study of chomosome behavior in gametogenesis has now revealed what it is. We have seen that when the germ cells are formed, corresponding chromosomes of male and female origin meet during synapsis, and then become separated to pass into different germ cells. If now we make
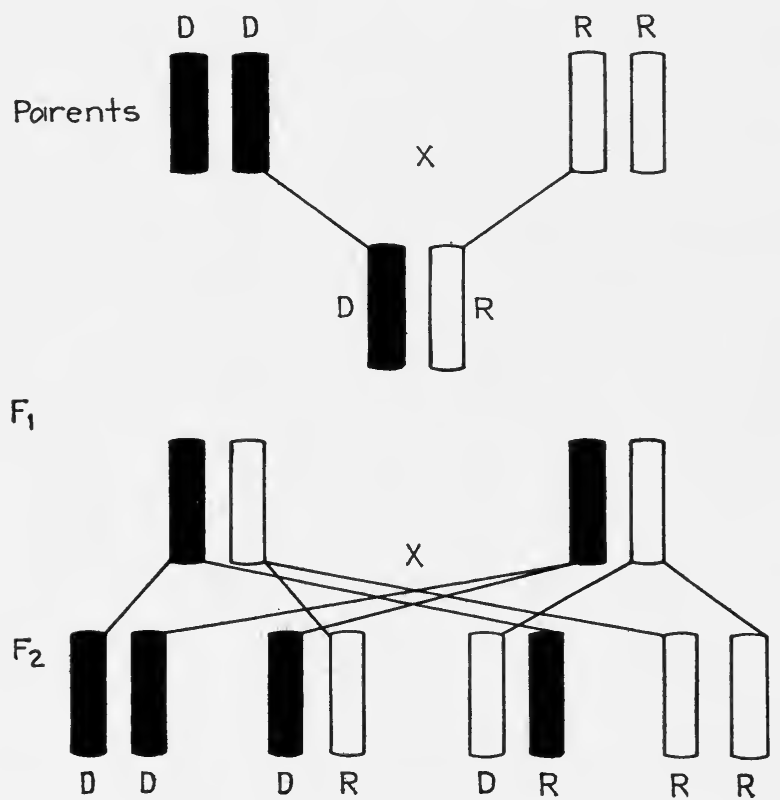

FIG. 17.-Gametes, or sex cells in Mendelian heredity. The parents $D D$ and $R R$ have each two factors for the dominant or the recessive character. Their sex cells will have only one such factor and the $F_{1}$ will therefore contain a dominant and a recessive factor from each parent. The progeny of the $F_{1}$ will have various combinations of the dominant and recessive factors in the ratio $1 D D+2 D R+\mathrm{I} R R$.

a very simple assumption that the factors for Mendelizing characters are borne by individual chromosomes, we have a very natural and plausible explanation of Mendelian heredity. According to this assumption, the germ cells would be pure for the factors for contrasted characters, and hence their random combinations would give rise to Mendelian ratios. In fact, Mendelian heredity may be said to be the inevitable outcome of the pairing of homologous chromo- 
somes during synapsis and their subsequent distribution to different germ cells.

In order to explain the typical dihybrid ( $9: 3: 3: 1)$ ratio, it may be assumed that the factors for different pairs of characters are borne by different pairs of chromosomes, each of which is assorted independently of the other in gametogenesis. We may then represent what takes place in crossing a round yellow with a wrinkled green pea somewhat as follows: Each plant we may assume is pure breeding, or homozygous and has two sets of chromosomes. Each character such as round or green is represented by something occurring in both members of one pair of chromosomes and hence in all the germ cells produced by the plants. In the round yellow peas, we may assume that the factors for round are carried by one chromosome pair, and those for yellow by another. We may suppose that wrinkled green peas are like the round yellow ones except that the factors for yellow and round are replaced by the factors for wrinkled and green in the corresponding pairs of chromosomes. Now when the two varieties are crossed, the $F_{1}$ hybrid has in all its cells factors for round, wrinkled, yellow, and green, and when the germ cells of the hybrid are being formed, the chromosome with the factor for round, and the chromosome with the factor for wrinkled pair in synapsis. And at the same time the chromosome with the factor for yellow pairs with the chromosome containing the factor for green. When these chromosomes separate and go into different germ cells, the chromosome having the factor for yellow and the chromosome having the factor for round may happen to go into one germ cell, and the two chromosomes with the factors for green and wrinkled into the other, or else, as is equally likely, the chromosome with the factor for yellow and the chromosomes with the factor for wrinkled may go into the one cell and the chromosomes with the 
factors for round and green into the other. As a result the $F_{1}$ hybrid will produce four kinds of germ cells in equal numbers as follows: Round yellow, round green, wrinkled yellow, wrinkled green.

When two $F_{1}$ plants cross, or if an $F_{1}$ plant produces seed by self-fertilization, each of the four kinds of female germ cells may unite with any of the four kinds of male germ cells, thus producing sixteen combinations. One common way of determining what combinations are likely to be formed in the second generation of a cross is to write the formulas of the germ cells of one parent along the top of a square and the germ cells of the other along the left side. Then the square is divided into smaller squares according to the number of different kinds of germ cells involved, and the various combinations are written in each square. In representing genetic factors it is customary to designate the dominant ones by large letters and the corresponding recessives by small letters, thus $R$ would stand for round, $r$ for wrinkled, $Y$ for yellow, $y$ for green. A pure, round yellow pea would be $R R Y Y$, a wrinkled green pea rryy, and the $F_{1}$ hybrid $R r Y y$. Using this nomenclature we may work out the expected $F_{2}$ generation by the checkerboard method as is illustrated in Fig. I6. In working out the expected results of any mating the procedure is: first to ascertain the possible gametes produced by the parents, and then to find the various combinations which these gametes form. For instance, if we wished to know what would probably result from crossing the $F_{1}$ in our example back to the green wrinkled parent, we take the four types of gametes produced by the $F_{1}$ and combine each with the one type of gamete ( $r y)$ produced by the wrinkled green pea. The result will be four kinds of progeny, round yellow, round green, wrinkled yellow, and wrinkled green in equal numbers. 
In order to get a real grasp of the principles of Mendelian heredity, it is necessary to work out a number of problems. One does not acquire proficiency in algebra or geometry without solving problems, and it is much the same with genetics. Accordingly several questions for solution are given at the end of the chapter.

If we would form a picture of the hereditary mechanism, say of a human being, we should represent first a double set of chromosomes forty-eight in number, of which twentyfour are derived from the father and twenty-four from the mother. Carried somehow in these chromosomes are the factors involved in the production of hereditary characteristics. These are called genes. We look upon genes as little self-perpetuating units. We may say that all genes come from antecedent genes, just as all cells come from other cells. Each cell of the body contains all the genes that were originally present in the fertilized egg, from which the body cells were derived. How genes act to produce characters we do not know. A pea giving rise to wrinkled seed may differ from a pea producing round seed only in a single pair of genes out of possibly thousands. We may suppose that the gene for wrinkled peas produces an enzyme or ferment that transforms some of the starch of the seed into sugar. In some way particular genes must affect the surrounding cytoplasm, causing it to change in some specific way, and hence to produce a visible change in the organism. Different genes produce very different effects, probably in most cases in a very indirect way. One gene may cause a small pit in the human ear, which has been observed to be transmitted through several generations, another may cause an individual to have six fingers instead of five, and another may cause its possessor to develop Huntington's chorea late in life and die of convulsive seizures. Manifold are the effects of genes. The cooperative action of a large number of genes 
is required to build up an organism, but it not infrequently happens that genes cause deviations from the normal, as in Huntington's chorea, which are anything but advantageous.

Where genes produce such marked departures as to cause the death of the organism, they are called lethal factors. Many kinds of lethal factors have been identified in different organisms. In some varieties of Indian corn, one-fourth of the seedlings developed no chlorophyll and formed weak albino plants that soon died. The lack of chlorophyll depends upon recessive genes that exercise a lethal effect by preventing the plant from building up its carbohydrates under the influence of sunlight. In a strain of fruit flies, a part of the larvae developed pigmented tumors that have a fatal effect, and in horses a recessive factor has been described that causes a closure of the intestine with fatal results to the young colts soon after they are born. Lethal genes produce their fatal effects in many ways just as other genes produce many kinds of normal variations. Among the children of the family all drawing their genes from the common lottery of the parental germ plasms, Mary may have red hair, John hare lip, William may have epileptic fits, Polly's second and third toes may be connected by a fleshy web, and Algernon may be a musical prodigy. The shuffling and sorting of the chromosomes produce almost no end of diverse combinations of hereditary traits. If a man and his wife were to have a thousand children instead of a maximum of less than thirty probably no two of them, unless they happened to be identical twins, would have the same hereditary endowments.

\section{Suggested Readings}

Jennings ('30), chaps. I and 2. Shull (' 3 I), chaps. 7-9. Sinnott and Dunn ('32), chaps. 3, 4. Wells, Huxley, Wells ('29), book 4, chap. 4. Further discussions in Babcock and Clausen ('27), Altenberg ('28), Conklin ('30), Dunn ('32), Jennings ('35), Morgan ('19), ('28). 


\section{Questions}

I. In guinea pigs rough hair $R R$ is dominant over smooth $r$. What is the genetic formula of the $F_{1}$ of a cross between a rough and a smooth guinea pig? What germ cells does the $F_{1}$ produce? What will be the second, or $F_{2}$ generation?

2. What will result from crossing the $F_{1}$ back to the white parent? to the black parent?

3. A cross between a white and a black guinea pig gives seven black and six white offspring. What is the genetic composition of the parents?

4. Crossing two peas gives 298 round and Ior wrinkled seed. What was the genetic make-up of the parents? What character is dominant?

5. What part does synapsis play in securing the purity of the gametes for contrasted Mendelian factors?

6. Would you expect Mendelian inheritance in forms reproducing asexually?

7. Would you expect Mendelian inheritance if chromosomes did not preserve their individuality?

8. Why are apples, peaches, and many other fruits propagated by buds or grafts? What commonly happens when these fruits are raised from seed? Why?

9. In guinea pigs black is dominant over white. If a rough black is crossed with a smooth white guinea pig what will be the $F_{1}$, the germ cells of the $F_{1}$, and the proportions of the different types in the $F_{2}$ ?

I0. Make a diagram of the chromosomes of the rough black and the smooth white guinea pigs, putting the factors for rough, $R R$, or smooth, $r r$, in one pair of chromosomes, and the factors for black, $B B$, or white, $b b$, in another pair. Diagram the chromosome make-up of the $F_{1}$ and the four types of germ cells of the $F_{1}$.

II. What will result from a cross between the $F_{1}$ and the smooth white parent? between the $F_{1}$ and the rough black parent?

What will be the immediate progeny of a cross between guinea pigs of the following genetic formulas?

$$
\begin{aligned}
R R B b & \times r r B b \\
r r B b & \times R r b b \\
R R b b & \times R r B B \\
R R B B & \times r r B b
\end{aligned}
$$

12. In man brown eyes are dominant over blue. A brown-eyed man with a blue-eyed mother marries a woman both of whose parents were blue-eyed. What would be the expected eye colors of the children? 
13. If these parents had four children what is the chance that they would all be blue-eyed?

14. Does the fact that three children in such a family are blue-eyed affect the probability that the fourth child will be blue-eyed?

15. Stubby fingers (brachydactylism) is a dominant character. What is the chance that the child of a normal woman and a stubby fingered father will inherit the trait if the father is heterozygous? 


\section{CHAPTER IV}

\section{HEREDITY AND SEX}

NE very important outcome of the study of gametogenesis is the solution of the age-long riddle of the determination of sex. A very large number of organisms resemble the human species in producing males and females in approximately equal numbers. One might be prone to regard this as a providential arrangement in the interest of monogamous unions but we find it among animals who are quite promiscuous in the matter of mating, as well as in polygamous species in which an old and powerful male monopolizes most of the females and drives away all rivals.

This approximate numerical equality of the sexes long remained an unexplainable fact. The first real light began to dawn when it was discovered that in the spermatogenesis of certain species of insects there is an odd, or unpaired chromosome which fails to divide, and is passed bodily into one or the other of the two spermatocytes. In I902 McClung advanced the theory that this odd chromosome is a determiner of sex, since half of the sperm cells contain this chromosome and half do not. There are many species in which the odd, or $X$-chromosome instead of having no mate has a mate of smaller size called the $Y$-chromosome. Apparently it matters little whether a $Y$-chromosome is present or not. The important point is, in any case, that sperm cells fall into two classes, half of them containing the $X$-chromosome and the other half containing the $Y$-chromosome or no $X$-chromosome.

A startling theory, such as McClung's, did not have to wait long before being tested. Not only were sex chromo- 
somes studied in a large number of species both plant and animal, but attention was turned to the study of the chromosomes in the body cells of both sexes. It was found that in the formation of the mature egg cells, all the eggs receive the same number of chromosomes, and that an $X$-chromosome was present in every mature egg. It is only the sperm cells that fall into two classes. This being so, it was natural to suppose that if an egg was fertilized by a sperm containing an $X$-chromosome, it would produce a female, and if fertilized
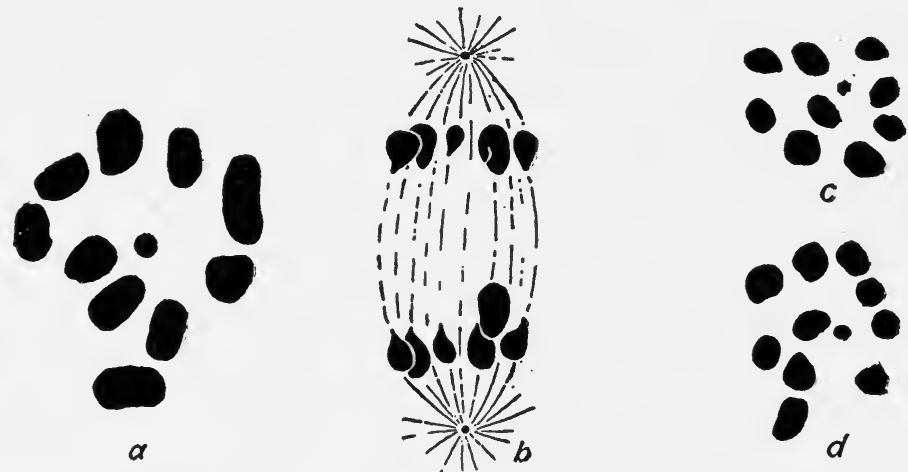

Fig. 18.-Sex chromosomes of the male bug Anasa. $a$, polar view of the division of the first spermatocyte; $b$, side view of the second division showing the large sex chromosome passing undivided into the lower cell; $c$ and $d$ polar views of the last division showing ten chromosomes in one group and eleven including the undivided sex chromosome in the other. (After Wilson.)

by sperm without an $X$-chromosome, but only a $Y$, it would produce a male. Here was a very neat and simple theory, almost too simple to be true. And cytologists began to turn their microscopes on the cells of the male and the female of the species in order to count their chromosomes. It turned out that in most species studied, the females have an even number of chromosomes, including two $X$-chromosomes, whereas the males have only one $X$-chromosome, in some species with, and in others without, a $Y$-chromosome. These facts lent strong support to the view 
that the chromosome differences of the sexes are really responsible for the determination of sex.

A large amount of research on the chromosomes of sex cells and body cells has yielded abundant confirmation of this hypothesis. Sex determination would then appear to be settled at the time of fertilization, when the individual receives his or her allotment of chromosomes. All the theories as to how the sex of unborn offspring could be changed at will seemed to be swept away at once.

In most animals, man among the rest, and in some plants it has been found that it is the male which produces two kinds of gametes, or is the heterozygous sex. The birds and the Lepidoptera (butterflies and moths) among insects are exceptions to this rule in that the male is homozygous and the female heterozygous for sex determining factors. These facts have thrown much light upon the interpretation of what is called sex-linked heredity. Certain hereditary characteristics such as the horns in male deer, and the mammary glands of the female are regularly associated with sex and are known as secondary sexual characters, but sexlinked characters are transmitted in a somewhat different way. Let us take an illustration from human heredity. If a normal woman marries a color-blind man, all her children, male and female, will be normal, but her daughters may transmit color blindness to their sons. The gene for this trait is recessive and does not appear in the presence of its corresponding normal factor. The peculiar inheritance of color blindness is readily explained simply by assuming that its gene is carried by the $X$-chromosome. The daughters of a color-blind man and a normal woman have the defective gene in the $X$-chromosome derived from the father, and a normal gene in the $X$-chromosome derived from the mother. Although not showing the trait themselves, the daughters would bequeath the gene to half their sons who, having but 
one $X$-chromosome and no normal gene to counteract its influence, develop color blindness. Sex-linked characters thus present an exception to the rule that recessive traits can manifest themselves only in a homozygous individual. The fruit fly, Drosophila melanogaster, has over two hundred sex-linked characters. In man there are much fewer sexlinked characters known. Among these is hemophilia, a condition in which the affected person bleeds very readily owing to the imperfect clotting of the blood.

According to the usually accepted opinion, each sex may carry factors for the opposite sex as well as its own. Like other characters sex is a matter of genic balance. Males derive their $X$-chromosome from their mother, and females derive one of their $X$-chromosomes from their mother and the other from their father. It is a curious fact that in the honeybee, the eggs which are unfertilized and which derive their chromosomes exclusively from the female develop into drones, or males, while the addition of the chromosomes of the male in the eggs which are fertilized results in the production of females. Two doses of the $X$-chromosome produce a female, and only one produces a male. Apparently sex is determined ordinarily by the quantitative relation between the amount of $X$-chromosome material to the rest of the germ plasm. This conclusion is strikingly borne out by the fact that in Drosophila it has been found that occasionally there are two $X$-chromosomes and three sets of the other chromosomes, in which case the individual is more or less intermediate between a male and a female, and is called an intersex. It has been supposed that the $X$-chromosome contains factors that tend to make an individual a female and that the male-producing factors are in the other chromosomes. Where one $X$-chromosome is present there are two male-producing sets of factors $(2 M)$ present and the result is a male. Where there are $2 F$ and 

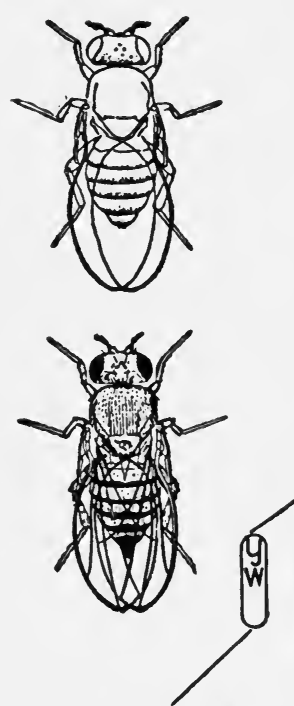

周周
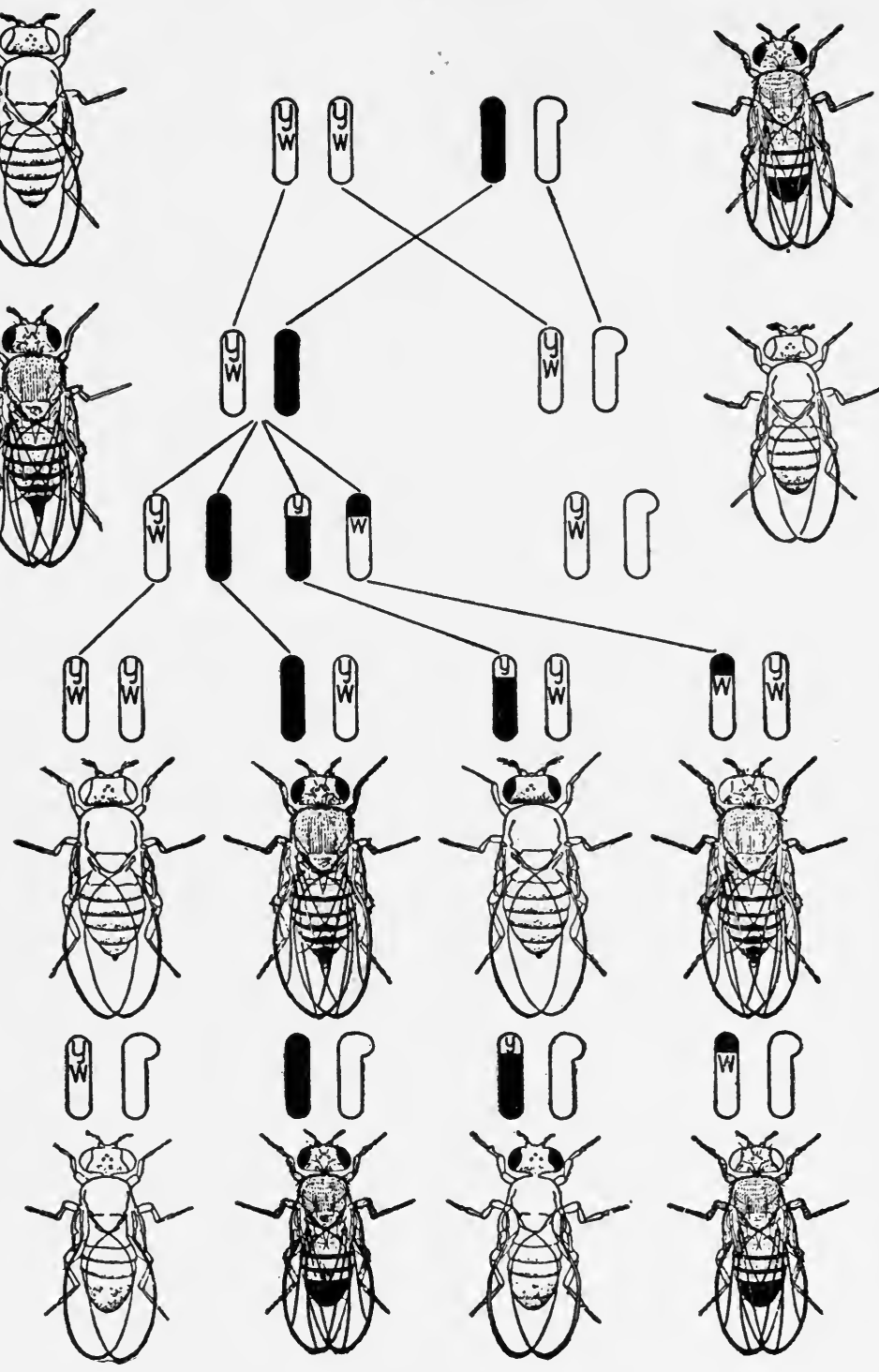

Fig. 19.-Sex-linked heredity in Drosophila. A yellow white-eyed female is crossed with a gray, red-eyed male. The immediate $\left(F_{1}\right)$ progeny consist of gray, red-eyed females and yellow, white-eyed males. In the $F_{1}$ female crossing over occurs in some of the gametes so that the yellow and white factors previously linked become separated and lie in different gametes. The result of crossing the $F_{1}$ female back to the original male parent is to produce the eight types indicated below. (After Morgan.) 
$2 M$ producing factors, the balance is turned toward the female sex. Where the ratio is $2 F$. to $3 M$, a more nearly intermediate condition is presented and results in an intersex. According to this interpretation sex determination is really a matter of dominance. In most animals we may say that $2 F$ is more potent than $2 M$, and that $2 M$ is more potent than $I F$. In the birds and the Lepidoptera, $2 M$ is more potent than $2 F$, but $2 F$ is more potent than I $M$.
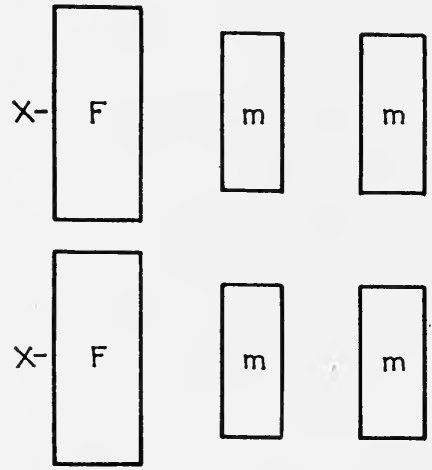

Female

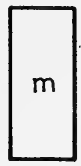

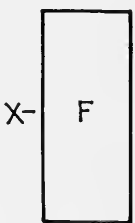
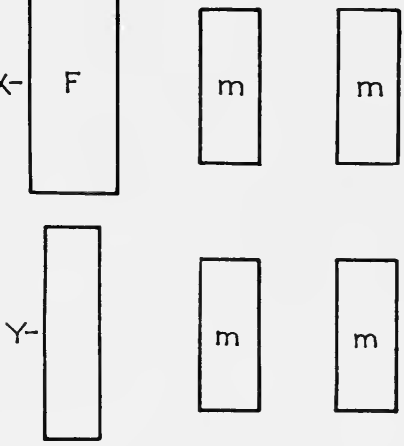

Male

FIg. 20.-Diagram illustrating the distribution of sex determining factors in the chromosomes. The factors for femaleness are assumed to lie in the $X$-chromosome, those for maleness in the other chromosomes, or autosomes. Two $F$ factors in the $X$-chromosomes outweigh the two sets of $m$ factors in the autosomes and produce a female. Where there is only one $X$-chromosome the two sets of $m$ factors outweigh the one $F$ and produce a male.

The fact that each sex contains both sex factors not only helps us to explain the phenomena of sex inheritance and the exceptional occurrence of intersexuality, but it enables us to understand why the sex of an organism can sometimes be changed. In some hermaphrodite animals (i.e., those having both male and female sex organs in one individual) the sex may be at first male and later female. The common eastern oyster regularly changes its sex with the seasons. Gould has shown that the free-swimming larvae of the slipper shell Crepidula which settle down near an adult female become males. Otherwise, after passing through a 
more or less hermaphroditic period, they develop into females. Probably some substance given off by the female affects the sex differentiation of the young Crepidulas. In this way the mollusks which are situated near together are caused to be of opposite sex, and hence the eggs which are discharged from the female are more apt to be fertilized. A similar case is afforded by the marine worm Bonellia. In these forms environment determines whether an individual develops into a male or a female. Each embryo contains the genetic factors for both sexes, and external conditions decide which kind of sex factors assume the predominant role in development.

In organisms having sex chromosomes the determination of sex is largely independent of the environment and is definitely established at the time of fertilization. There are some forms, however, in which the potencies of the opposite sex determining factors appear to be more nearly balanced, so that under exceptional circumstances the influence of the chromosome complex may be overcome. A few years ago Dr. F. A. E. Crew described a hen which, after having laid eggs, developed the plumage and peculiar behavior of a rooster. Later she (or he) became the father of several chicks. A post mortem examination showed that the ovary had been nearly destroyed by tuberculosis and that testicular tissue had developed in its stead and produced spermatozoa. A little later Riddle described a case of transformation of sex in pigeons due also to ovarian infection with tuberculosis. Then followed experiments by Domm and others to ascertain if the sex of fowl could be changed by the castration of young females. In birds there is only one functional ovary, the left, the right sex gland being represented by a small rudiment. It was shown that early removal of the functional ovary caused this rudiment to increase in size and, in many cases, to develop into a testis, although varying amounts 
of ovarian tissue may regenerate at the same time. Where the newly formed tissue is mostly testicular there is a fair'y complete transformation of sex. Within certain limits, therefore, early castration may cause females to change into males. A somewhat analogous case is found in toads. If a male toad is castrated a rudimentary structure called Bidder's organ (really an abortive ovary) enlarges and becomes transformed into a true ovary, which may produce eggs capable of fertilization and development.

It is now definitely established that the sex of frogs can be determined by temperature. Under ordinary circumstances frogs' eggs produce males and females in about equal numbers. In many cases young frogs pass through a hermaphrodite period in which rudiments of both male and female sex organs occur in the same individual. Later either testicular or ovarian tissue predominates and the individual becomes exclusively male or female. Sometimes the hermaphrodite condition persists until adult life, and there are several instances of such individuals which have produced functional eggs and sperm. Witschi has shown that when the eggs of the wood frog are subjected to a high temperature the sex organs of those which are genetically females develop into testes and all the frogs become males.

In most animals a change in the sex glands causes a change also in the secondary sexual characters. Among many species, including the higher vertebrates, changes in secondary sexual characters are largely effected through the influence of the sex hormones, or internal secretions of the sex glands. A striking illustration of this fact is afforded by the experiments of Steinach in transplanting the sex glands of young rats. Male rats are distinguished from females by their larger size, coarser hair, and greater pugnacity. Steinach found that if the testes of young male rats are removed and ovaries grafted in their stead, the rats 
attain about the size of females and do not develop the coarse hair and pugnacious disposition characteristic of the male sex. Their mammary glands became functional also and in some instances they gave suckle to young. Females in which the ovaries had been replaced by testes developed the size, coarse hair, and sex instincts of males.

These changes in structural characters and in instinctive behavior demonstrably result from the bodily influence of hormones secreted by the sex glands. When these glands are incompletely or abnormally developed the condition is often manifested by changes of external appearance and activities. It is a curious fact that crosses between different varieties or species sometimes result in various degrees of intersexuality. Within each group male-producing and female-producing factors have developed degrees of potency which ordinarily insure approximately equal numbers of the two sexes. When varieties are crossed the normal balance of sex determining factors may be disturbed thus causing the appearance of an intersexual condition. How far varied combinations of genetic factors may influence the degree of sexual differentiation in human beings is uncertain. There are masculine types of women and feminine types of men. Neither class is particularly attractive to most normally constituted persons. It seems likely, therefore, that the choice of mates in the human species has a tendency to maintain, if not to increase, the distinctive peculiarities of the two sexes.

\section{Suggested Readings}

Lindsey ('32), chap. 9. Shull ('31) chaps. I4, I 5. Sinnott and Dunn ('32), chap. 9. Wells, Huxley, Wells ('29), book 4, chap. 6.

\section{Questions}

I. In what way does the inheritance of sex resemble that of a cross between heterozygote, $D R$, and a double recessive, $R R$ ? 
2. What would happen to a female if one of her $X$-chromosomes disappeared before the first division of the egg?

3. In plants which produce both ovules and pollen is sex determined by differences in chromosomes?

4. If a character depends upon a gene in the $Y$-chromosome how would it be inherited?

5. A color-blind man marries a normal woman and one of her daughters marries a normal man and the other daughter marries a color-blind man. Give the expected distribution of color-blindness in the children of each mating.

6. In what way does the inheritance of such a character as the beard differ from that of a sex-linked character?

7. Suppose that a sex-linked character is dominant, as in some forms of night blindness in man. What will be the progeny, male and female, of a normal male and an affected (heterozygous) female? of a normal female and an affected male? of one of the daughters of the latter union and a normal male?

8. Without sexual reproduction would there be any Mendelian segregation?

9. Why does a recessive sex-linked character appear in the male and only rarely in the female?

10. What reasons can you give as to why in some species one sex is more numerous than the other?

II. What forms would be more variable, those produced sexually or those produced asexually? Why?

12. What is Darwin's theory of sexual selection?

13. Both hemophilia (bleeding) and atrophy of the optic nerve are recessive sex-linked characters in man. What would be the result of mating a man having optic atrophy and a woman having hemophilia? Of one of the daughters of this union with a normal man? 


\section{CHAPTER V}

\section{THE INTERACTION OF FACTORS}

\} { } ^ { \mathrm { E } } \mathrm { COME } \text { now to a topic of the greatest importance } for the proper comprehension of the workings of heredity. First of all it is essential to bear in mind that characters are never transmitted per se, but they are always a product of a large number of genes. When we speak of characters that behave like units in inheritance, one may easily gain the impression that each character is caused by a particular gene, much as each kind of plant is produced by a certain kind of seed. The analogy, however, is apt to be misleading. Geneticists have pretty much given up talking about unit characters. They speak instead of unit factors. A single factor may cause the difference between brown and blue eyes, but this is very different from saying that blue eyes are caused by a single gene. There are demonstrably many genes which, in one way or another, affect the development of the eyes. When some of these are modified they may cause cataract, color blindness, misplaced lens, atrophy of the optic nerve, or a multitude of other hereditary or partly hereditary defects of the eyes, whether they are blue or brown. Genes are differential factors. Alter a single gene and the blue eyes which might thus arise would still be the result of the coordinated workings of perhaps hundreds of genes.

There are several classes of genetic factors, distinguished according to how they act in the development of a given feature of the organism. Sometimes there are two or more factors in different pairs of chromosomes which produce 
much the same kind of effect. These are commonly called multiple factors. When, for instance, a certain brownseeded variety of oats was crossed with a white-seeded variety, the second generation of hybrids was found to consist of about fifteen browns to one white. This result

\begin{tabular}{|c|c|c|c|c|}
\hline & $B_{1} B_{2}$ & $B_{1} b_{2}$ & $b_{1} B_{2}$ & $b_{1} \quad b_{2}$ \\
\hline$B_{1} B_{2}$ & $\begin{array}{l}B_{1} B_{2} \\
B_{1} B_{2}\end{array}$ & $\begin{array}{ll}B_{1} & b_{2} \\
B_{1} & B_{2}\end{array}$ & $\begin{array}{ll}b_{1} & B_{2} \\
B_{1} & B_{2}\end{array}$ & $\begin{array}{ll}b_{1} & b_{2} \\
B_{1} & B_{2}\end{array}$ \\
\hline$B_{1} b_{2}$ & $\begin{array}{l}B_{1} B_{2} \\
B_{1} b_{2}\end{array}$ & $\begin{array}{l}B_{1} b_{2} \\
B_{d} b_{2}\end{array}$ & $\begin{array}{ll}b_{1} & B_{2} \\
B_{1} & b_{2}\end{array}$ & $\begin{array}{ll}b_{1} & b_{2} \\
B_{1} & b_{2}\end{array}$ \\
\hline$b_{1} B_{2}$ & $\begin{array}{l}B_{1} B_{2} \\
b_{1} B_{2}\end{array}$ & $\begin{array}{ll}B_{1} & b_{2} \\
b_{1} & B_{2}\end{array}$ & $\begin{array}{l}b_{1} B_{2} \\
b_{1} B_{2}\end{array}$ & $\begin{array}{ll}b_{1} & b_{2} \\
b_{1} & B_{2}\end{array}$ \\
\hline$b_{1} b_{2}$ & $\begin{array}{l}B_{1} B_{2} \\
b_{1} b_{2}\end{array}$ & $\begin{array}{l}B_{1} b_{2} \\
b_{1} b_{2}\end{array}$ & $\begin{array}{l}b_{1} B_{2} \\
b_{1} b_{2}\end{array}$ & $\begin{array}{l}b_{1} b_{2} \\
b_{1} b_{2}\end{array}$ \\
\hline & & & 01 & \\
\hline
\end{tabular}

FIG. 21.-Diagram illustrating the inheritance of brown color when it is due to two pair of factors which segregate independently. Where there is one or more factors for brown $B_{1}$ or $B_{2}$, the progeny are brown. Only one of the 16 combinations, $b_{1} b_{2} b_{1} b_{2}$, is white.

was shown to be due to two independent pairs of factors for brown. The $15: 1$ ratio is a modification of the typical $9: 3: 3: 1$ ratio in that the first three groups all look alike because they all have at least one of the dominant factors for brown color. In a cross between a red and a white variety of wheat, the second generation was found to yield sixty-three 
reds to one white. Here the result was shown to be due to three independent factor pairs for color. Among the sixty-three colored class some have more factors for dark color than others, but where dominance is nearly complete these cannot be distinguished by inspection. In some cases of multiple-factor inheritance the results are cumulative, the indvidual having the largest number of factors showing the character developed to the highest degree. Apparently something like this occurs in the skin color of crosses between Negroes and whites. The genes for black pigment are not completely dominant over those for lighter color, and the first generation of mulattoes is intermediate in shade. The children of the mulattoes, however, are apt to have varied combinations of black and white genes and hence they exhibit a considerable range of skin color from nearly black to nearly white.

The discovery of multiple-factor heredity has afforded a very plausible solution to one difficulty which sorely puzzled the early Mendelians. In some cases the $F_{1}$ generation is intermediate between the two parental types and gives rise to progeny which are also more or less intermediate. Crosses between large and small varieties of rabbits result in the formation of an intermediate and fairly stable type. Some geneticists were disposed to distinguish between two radically different kinds of heredity, the one following Mendel's law, the other resulting in so-called blending inheritance. The analysis of several cases of multiple-factor heredity gave rise to the hypothesis that what appear to be permanent blends are really the result of many factors having somewhat similar bodily effects. The multiple-factor hypothesis is supported by the general fact that the second generation of hybrids, as in the offspring of mulattoes, is more variable than the first, thus revealing a tendency to the segregation of factors which, on account of their large 
numbers, rarely produce again quite the same combination found in the original ancestors. As thus interpreted blending inheritance is essentially Mendelian. This opinion which is supported by many experimental tests is held by most geneticists at the present time. Altogether, it seems to be the most reasonable hypothesis in the light of the genetic evidence and what is known of the cytological basis of heredity.

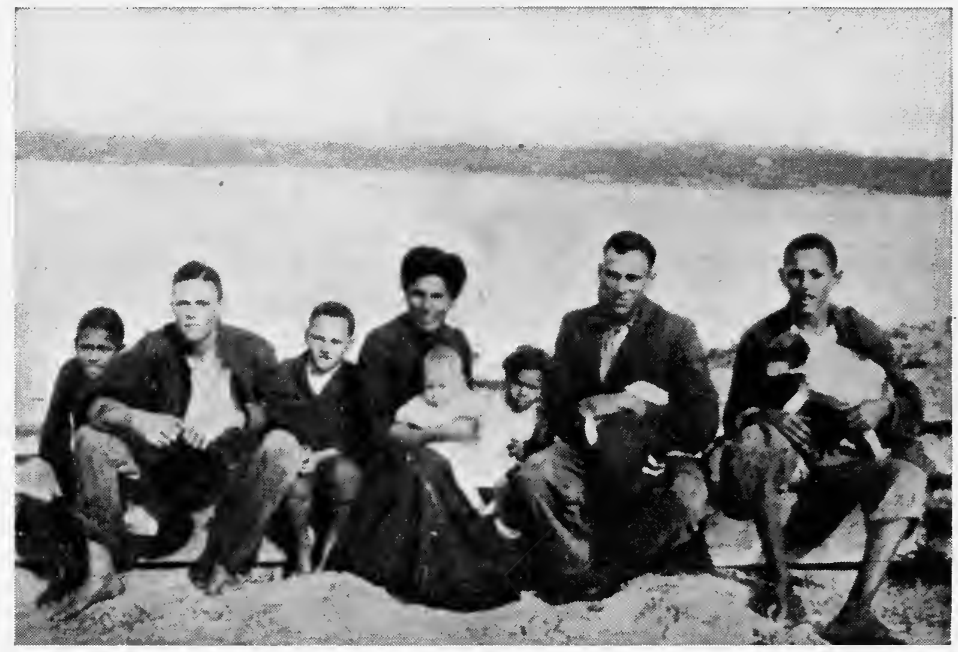

Frg. 22.-Variability of skin color in the offspring of mulattoes. (After Davenport. Copyright by Fournal of Heredity.)

There is a class of genes called modifying factors, because they affect more or less the expression of certain characters. Thus in fruit flies having eosin-colored eyes there are several factors whose position has been located in diverse parts of the chromosome system which modify eosin color, making it a little darker or a little lighter. Each of these modifiers behaves like an independent Mendelian unit and has little effect, so far as can be determined, upon other kinds of eye color.

Then there are factors that are called complementary inasmuch as each alone produces no visible effect, but when 


\section{HUMAN GENETICS AND ITS SOCIAL IMPORT}

combined may cause a certain character to appear. A wellknown example of such factors is furnished by two purebreeding varieties of sweet peas which when crossed give rise to a purple and red flower similar to that of the ancestral Sicilian species from which our numerous cultivated varieties have descended. The Mendelian explanation of this curious
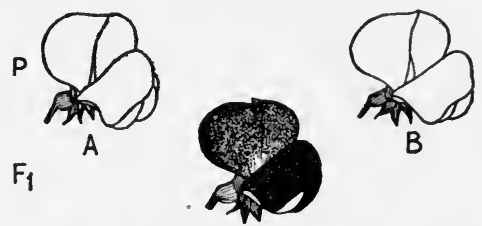

B
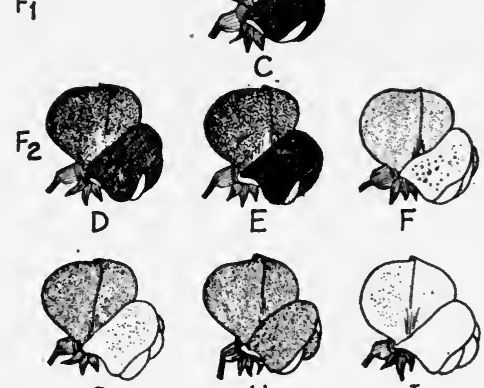

$\mathrm{H}$
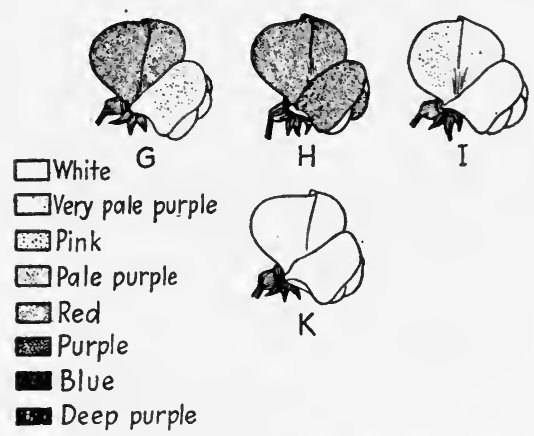

Fig. 23.-Results of crossing two races of white sweet peas, $A$ and $B$. The $F_{1}$ is purple like the ancestral Sicilian species. The $F_{2}$ presents a striking degree of variability as a result of the segregation of several factors for color. (From Conklin, after Punnett.)

phenomenon is that both white varieties lack something necessary for the production of color, but that they do not both lack the same thing. We may say that color requires the presence of two factors $C$ and $P$. If either is absent the flowers are white. The one pea we may represent as $C C p p$, and the other as $c c P P$. When their gametes $C P$ and $c P$ unite they bring together the complementary factors $C$ and $P$ required to produce color. There is this a restoration 
of the complement of factors present in the wild ancestral species.

The phenomenon of reversion has long been familiar to breeders of plants and animals. Darwin noted that crosses between different color varieties of pigeons sometimes produced birds of a bluish color with dark bars across the wings like the ancestral blue rock pigeon from which most of our domestic varieties have been derived. Again, the crossing of rabbits of different color, say an albino and a yellow, for example, might give rise to a rabbit of grayish color like that of the wild species. These mysterious reappearances of old ancestral characters were interpreted as due to something carried in the germ plasm in a latent or inactive state until as a result of some peculiar circumstance they were awakened to full epiphany. An understanding of Mendel's law now enables us to supply an explanation of this puzzling phenomenon. The hypothesis that reversion is caused by bringing together complementary factors is one that can be put to experimental tests, and it has been able to meet the theoretical requirements. Some cases of reversion are brought about by the outcropping of recessive traits that may have been obscured by more recently appearing dominant factors.

What may be called reversion by restoration is a not uncommon effect of the crossing of domestic varieties derived from a common source. In some varieties one genetic factor necessary to produce a character may become recessive or possibly lost; in another variety a different factor may become recessive or lost. The cross between the two may restore the ancestral combination. It may happen that the varieties come to differ in several factors. In such a case the $F_{1}$ produced by the cross may be heterozygous for several pairs of genes, and consequently the second, or $F_{2}$ generation may present a great diversity of characteristics. The purple sweet pea arising from the cross between two whites may 
produce a varied combination of colors among which are purples, reds, pinks, and the two ancestral white varieties. In the second generation colored and whites are in the ratio of 9 colored to 7 white. This is a modification of the $9: 3: 3:$ I ratio in which the three latter classes have the same appearance, although they are genetically different.

There are several modifications of the typical Mendelian ratios. We may have, as you have seen, a $15: \mathrm{I}$ ratio, and there may be also $9: 7,9: 3: 4,12: 3: 1,13: 3$, and $10: 6$ ratios. At first the occurrence of these unusual ratios proved disconcerting to the experimental breeder. When he finds them he has to invent a hypothesis by which they can be explained. But if he is a really good geneticist, he will not be content with a mere plausible theory. He will test his supposition as he can often do by appropriate crosses and see if his findings tally with theoretical expectations. In order to explain the varied results of breeding experiments, geneticists have been led to hypothecate several kinds of factors, i.e., multiple, modifying, cumulative, lethal, complementary, etc. By this means many apparently nonconforming cases have been explained in accordance with the fundamental principles of Mendelian heredity.

It is important to bear in mind that the various modifications of typical Mendelian ratios that have been observed are brought about by the ways in which genetic factors interact in the production of visible characters. The pairing and segregation of genes go on in precisely the same way whether the characters appear in one ratio or in another. One common source of difference in Mendelian ratios is variations in dominance. In some forms one member of a Mendelian pair does not completely dominate over the other, but produces a more or less intermediate condition in the heterozygous offspring. When a red and a white four-o-clock are crossed the $F_{1}$ is pink, inasmuch as the gene for red color 
does not completely suppress the influence of its white mate. The pink $F_{1}$ generation gives rise to progeny consisting of one red, two pink, and one white, the reds and the whites being both homozygous, and the pinks revealing their mixed composition by their intermediate color.

Sometimes the characters interact in such a way as to produce something different from either. An illustration of
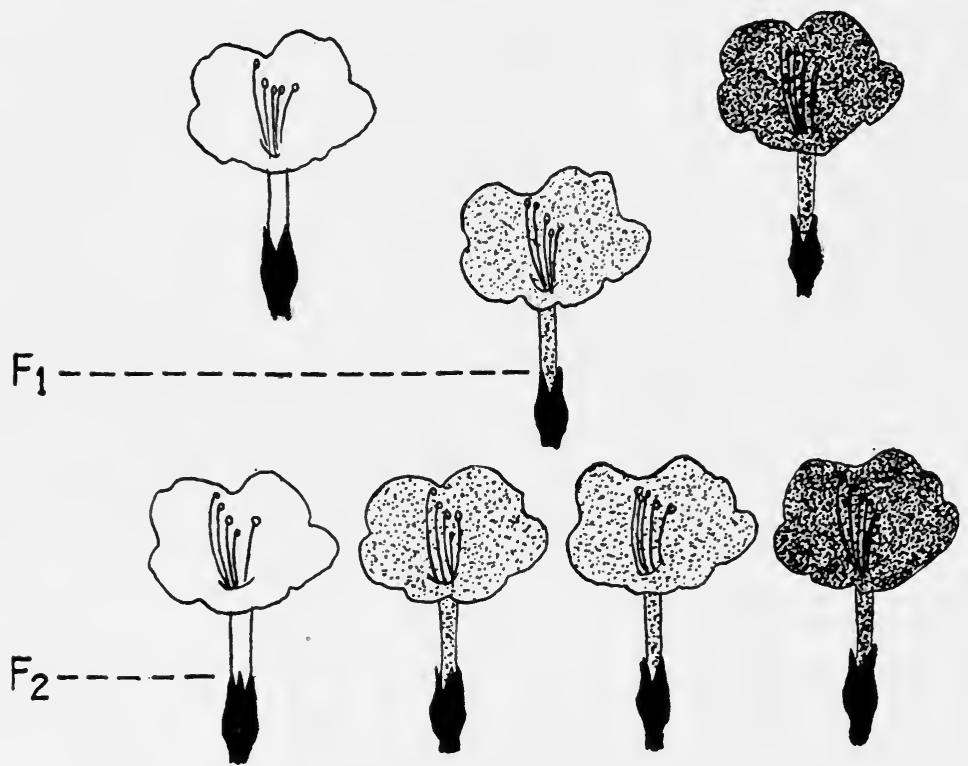

FIG. 24.- Incomplete dominance in the cross between a red and a white variety of four o'clock, Mirabilis jalapa. The $F_{1}$ of this cross is pink, but this heterozygous type forms in the $F_{2}$ generation reds, pinks, and whites in a $1: 2: 1$ ratio. (After Correns.)

this fact is produced by the blue Andalusian fowl which were long known never to breed true but to produce some black and white fowl in addition to blue. It was shown by Bateson and Punnett that the blue Andalusians were heterozygous, and that the only way in which to obtain nothing but blue birds was to cross blacks with whites. In some way the factors for white and black conspire to produce blue instead of either color alone or an intermediate shade. Fowls with pea 
comb and rose comb are pure-breeding varieties, but when crossed neither character is dominant over the other, both combining to produce the so-called walnut comb, which is quite different from either. Thus the interaction of complementary factors may give rise to quite unexpected novelties. How different gene combinations may conspire to produce

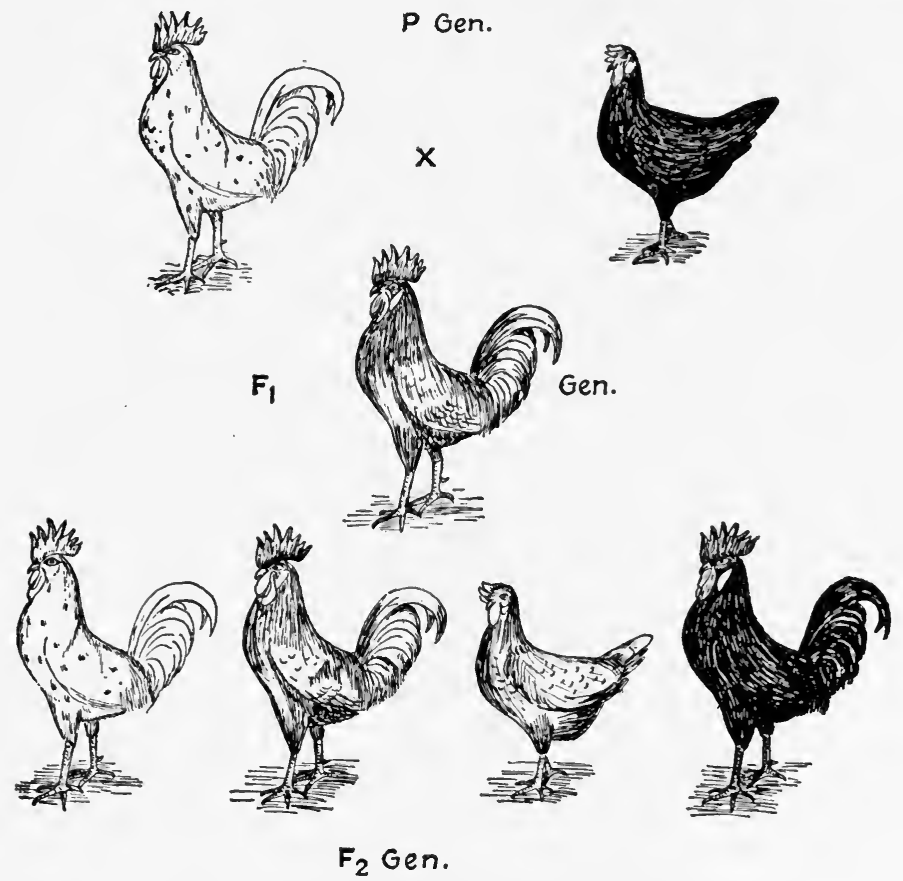

Fig. 25.-Andalusian fowl. (From Wolcott.)

visible characters in the developing organism no one can predict in advance. We are almost completely ignorant of the ways in which genes work to achieve their effects. We see only the end result of what may be a very long and complex chain of interactions.

The influence of a gene may depend not only upon other genes with which it is associated, but also upon environment. In some cases external factors may determine whether a 
character is dominant or recessive. In the fruit fly, Drosophila, a strain was obtained having an abnormal distribution of the color bands of the abdomen. Crosses with normal fruit flies produced offspring with abnormal abdomens, thus showing that the character behaves as a dominant. The degree of dominance was found to be subject to much variation, and if the flies were reared in a dry environment, the anomaly commonly failed to appear at all. In the proper environment
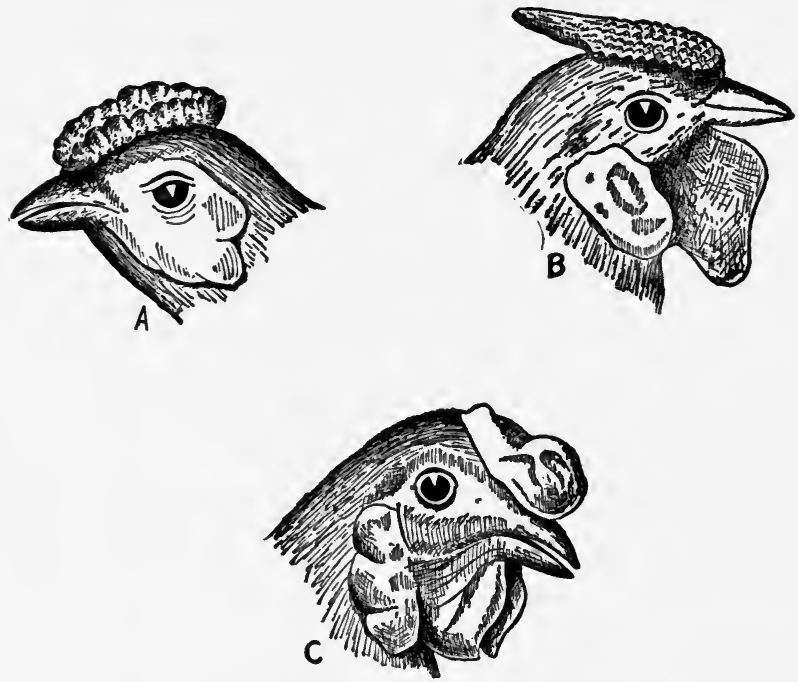

Fig. 26.-Inheritance of comb form in poultry. $A$, pea comb; $B$, rose comb, $C$, walnut comb, resulting from crossing $A$ and $B$. (After Shull.)

flies with the gene for abnormal abdomen are indistinguishable from ordinary flies. If these normal appearing flies are bred under usual conditions, however, their progeny will all reveal the presence of the defective gene. They differ from normal fruit flies in having a gene which gives them the capacity to respond to certain environmental stimuli by developing an abnormal abdomen.

There are many hereditary characteristics whose degree of expression is dependent upon surrounding conditions. A variety of Primula, $P$. sinensis rubra, produces red flowers 
at ordinary temperatures. When raised at a high temperature, its flowers are white, resembling those of a white-flowered variety, $P$. sinensis alba. Whether the plant inherits redness or whiteness, therefore, depends upon its environment. What it really inherits, of course, is the capacity to respond to a certain temperature by becoming red and to a higher temperature by becoming white. In this it is hereditarily different from $P$. sinensis alba, which is white at all temperatures.

Not infrequently a hereditary character may be developed to varying degrees even under what seem to be the same external conditions. Here dominance may be influenced by

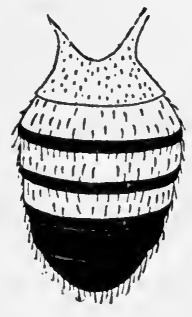

a
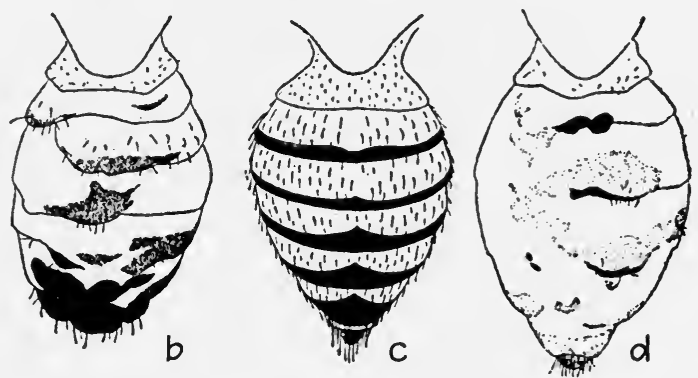

Fig. 27. - Normal and abnormal abdomen in Drosophila melanogaster. The normal condition in the male is shown in $a$ and in the female in $c$. The abnormal abdomens of the two sexes are shown in $b$ and $d$. (After Morgan by permission of the F. B. Lippincott Company.)

the presence or absence of other genetic factors, or by slight environmental differences which commonly escape notice. Polydactylism (supernumerary fingers or toes) ordinarily behaves as a dominant character both in human beings and in lower animals. The gene responsible for this anomaly is present in all cells of the body, but it often happens that only the hands or only the feet are affected, and frequently only one hand or one foot. The character is expressed in all degrees from six or more well formed digits to a mere supernumerary stub or protuberance. There are several cases in which normal individuals of polydactylous ancestry have produced polydactylous children. The fact that in pedigrees 
polydactylism sometimes skips a generation is doubtless to be attributed to the occasional failure of dominance. That the gene often fails to manifest itself in one hand or one foot makes it seem not unlikely that it would sometimes fail of expression altogether. In other words, some individuals may be genetically polydactylous and somatically normal.

Imperfect dominance is by no means a rare phenomenon. Numerous illustrations of it may be found in the inheritance of human traits. Families often manifest diatheses or proclivities to certain disorders without necessarily becoming affected. One may be prone to have diabetes or bronchial asthma, but in the absence of the proper exciting cause he

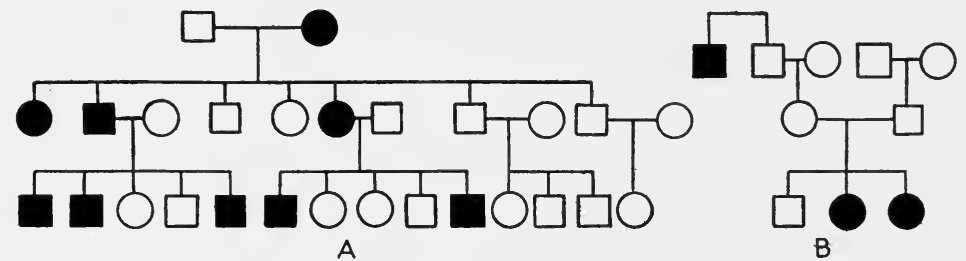

Fig. 28.-Two pedigrees of polydactylism. In pedigree $A$ the trait is clearly dominant. In pedigree $B$ since it skips a generation it is probably incompletely dominant. $(A$, after Lukas; B, after Koehler.)

may remain entirely normal. Some strains of mice are much more apt to contract pneumonia than others, and probably the same remark applies to human beings, but in the absence of the proper germs there can be no pneumonia. Sometimes hereditary immunities can be broken down by sufficient doses of virulent disease germs, or by weakening the resistance of the body through lack of food or exposure to cold.

Heredity is often looked upon as peculiarly fatalistic. It is true our allotment of genes is fixed at the time of fertilization. But if we inherit the gene for a certain trait it does not necessarily follow that we will have that trait. Within certain limits we may escape our heredity. One may be prone to develop insanity, but under proper condi- 


\section{HUMAN GENETICS AND ITS SOCIAL IMPORT}

tions the dread malady may never appear. There are, of course, many traits which we are powerless to evade. We cannot change the color of our eyes, or our membership in a given blood group. If a child were feeble-minded owing to an inherited deficiency of the thyroid gland, it might be converted into a person of normal intelligence by the administration of thyroid extract. That the defect can be overcome is no proof that it is not primarily due to heredity. Possibly future discoveries will place in our hands the means of counteracting many of our hereditary ills, but it would be hazardous to predict how much progress in this direction may eventually be made.

\section{Suggested Readings}

Shull ('31), chap. I6. Sinnott and Dunn ('32), chap. 5. See also references for chap. 3 .

\section{Questions}

I. What will be the result of crossing a blue Andalusian fowl with $(a)$ a white fowl, (b) a black fowl?

2. If an entirely white child were born to two mulattoes, would it be a pure Caucasian?

3. If all members of the Jones family developed bronchial asthma whenever they ate rye bread, what would you say of the probable role of heredity in causing the attacks?

4. Give three reasons why a character may skip one or more generations.

5. If when a defect occurs in a family it is found also in one-fourth of the brothers and sisters, what would you infer as to its mode of inheritance?

6. Yellow in mice is a dominant character and lethal when homozygous. Hence all yellow mice are heterozygous. What would be the expected progeny of a cross between a yellow mouse and a gray mouse?

7. If a sex-linked factor has a lethal effect, what would be the sex ratio in a cross between a heterozygous female and a normal male?

8. If a walnut comb fowl, $R R P P$, is crossed with a pea comb, $\operatorname{rr} P P$, what will be the expected progeny? What will result from crossing a walnut comb with a rose comb, $R R p p$ ? 
9. If the ovary is removed from a mallard duck, she develops the brilliant plumage of the male. Is this coloration an inherited character? If so, in what sense?

10. Tortoise-shell cats are almost always females and may be produced by crossing yellow and black. What would result from crossing a tortoiseshell female with a black male? with a yellow male?

II. Two shepherd's-purse plants with triangular pods give when crossed fifteen plants with triangular pods to one with oblong pods. What is the genetic constitution of the parents? Some of the progeny with triangular pods, when crossed, give three triangular to one oblong. How do you explain this result? 


\section{CHAP'TER VI}

\section{THE LINKAGE OF HEREDITARY TRAITS}

W

HILE most inherited characters are segregated out independently of others there are many exceptions to this rule. The first exception to the law of independent assortment was discovered by Bateson and Punnett, who found in crossing sweet peas that certain characters showed a marked tendency to be inherited together. This phenomenon, which is known as linkage, was later observed in the fruit fly by Morgan, who advanced the ingenious theory that linked characters depend upon factors borne in the same chromosome. The common fruit fly is admirably adapted for breeding experiments and the science of genetics owes a great debt to these small insects. They are easily raised by the thousands in small bottles. They breed rapidly, generations succeeding one another about every three weeks. They have few chromosomes, only four pairs, a very fortunate circumstance as it proved in studying linkage. There is a large pair of sex chromosomes, $X X$ in the female and $X Y$ in the male. There are two pairs of rod-shaped chromosomes of nearly equal size and a pair of small rounded fourth chromosomes. The work of Morgan and his able associates showed that the numerous heritable characters of Drosophila are inherited in groups, and that the number of linkage groups, i.e., four, exactly corresponds to the number of pairs of chromosomes. One large group is sex linked and hence probably associated with the $X$-chromosome. Two other large groups occur that are associated with the large second and third chromosomes, and one very small 
linkage group which, it was tempting to conclude, is carried by the small fourth chromosome. This supposition has been repeatedly verified by observing the genetic behavior of flies in which one of the fourth chromosomes had been lost or which possessed three of these chromosomes, or in

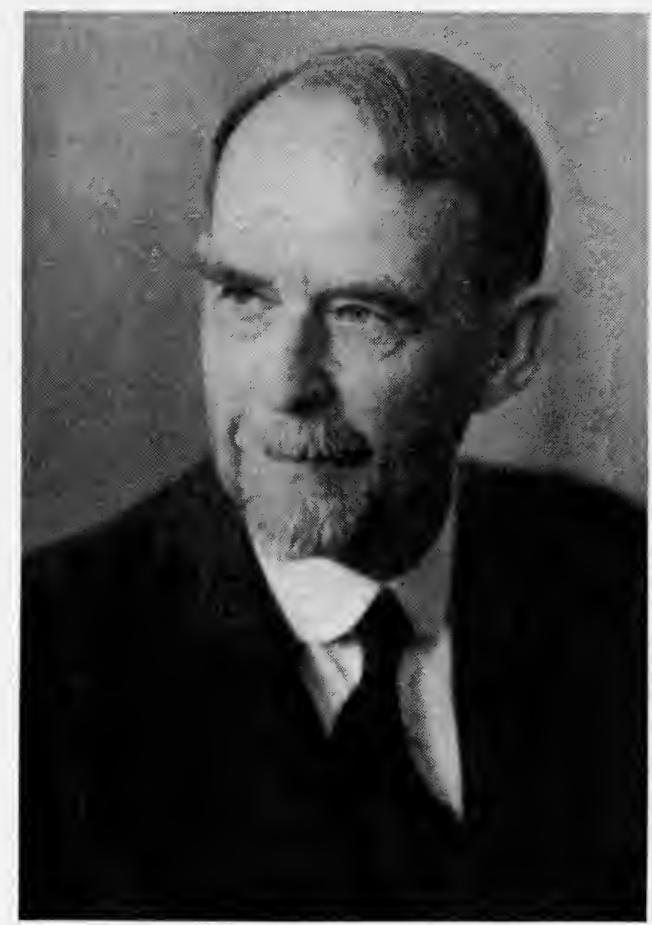

FIG. 29.-T. H. Morgan. (Copyright by the Fournal of Heredity.)

which a part or the whole of the fourth chromosome had become attached to one member of another pair.

One of the peculiar features occurring in most cases of linkage is that the association of the characters is not complete. For instance, when a fruit fly having long wings and gray body color was crossed with a fly having vestigial wings and black body color, the $F_{1}$ generation were all gray, long-winged flies (vestigial and black being recessive). 
'The $F_{1}$ females crossed back to the black, vestigial-winged male parent gave the four expected types, but they did not appear in equal numbers as they do in typical dihybrid crosses. Instead there were $4 \mathrm{I} .5$ per cent gray long, $4 \mathrm{I} .5$ per cent black vestigial, and 8.5 per cent gray vestigial, and 8.5 per cent black long flies. The characters entering the cross together were found to come out together to a greater extent than they would according to the principle of independent assortment. When the linked characters enter the cross in a
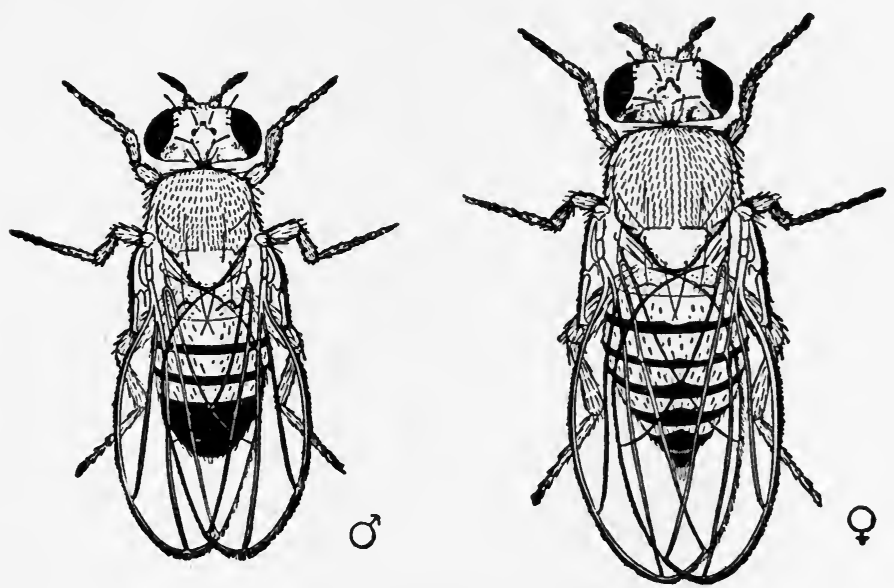

FIg. 30.-The fruit fly Drosophila melanogaster. Left figure male; right female. (After Morgan.)

different combination, as in crossing a gray, vestigial-winged with a black, long-winged fly, the $F_{1}$ female crossed with a black, vestigial-winged male produces $4 \mathrm{I} .5$ per cent gray vestigial, 4I.5 per cent black long, 8.5 per cent gray long, and 8.5 per cent black vestigial progeny. Linked characters, as these results show, are occasionally separated. But why? The associations are found to be exchanged in fairly definite ratios as in the experiments cited. Apparently, if linked genes are associated in the same chromosome they must somehow get exchanged occasionally, and it is natural to infer that the exchange occurs during the period of synapsis, when, as 
we have seen, there is a pairing of chromosomes of male origin with corresponding chromosomes of female origin.

The French cytologist Janssens in studying the spermatogenesis of the newt Batrachoseps had observed that during synapsis the paired chromosomes twist about each other. This observation suggested to Dr. T. H. Morgan that the exchange of linkage relations might be due to the fact that when the paired chromosomes separate they break apart at the points where they cross, and the broken ends of different chromosomes then unite and thus effect an exchange of segments. Hence the phenomena of crossing over observed

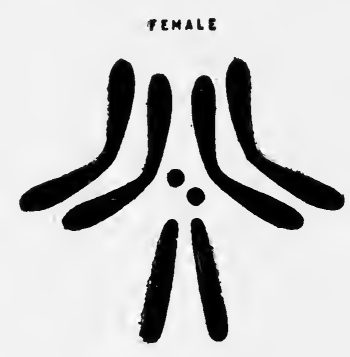

$x \quad X$

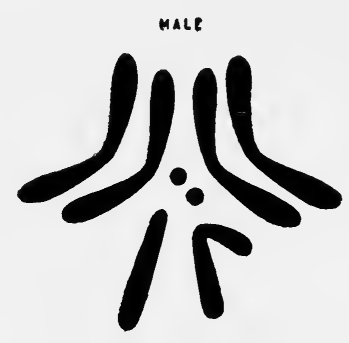

$\boldsymbol{X}$
$\boldsymbol{Y}$

Fig. 31.-The chromosomes of Drosophila melanogaster. $X X$ and $X Y$ represent the sex chromosomes. (After Bridges.)

in studying linked characters might be explained as due to an actual crossing over of segments of chromosomes. Both linkage and crossing over, therefore, are plausibly interpreted from a common standpoint.

It has been found that characters are linked in various degrees. In some instances crossing over is observed in only a fraction of I per cent of cases; in others, it occurs in almost 50 per cent, and hence approaches free assortment. These different degrees of linkage are explained by supposing that closely linked genes are situated close together in the chromosomes, so that it is unlikely that a break will occur between them. The farther apart the genes lie the more likely they will become separated. Upon this hypothesis it is possible 
to construct a chromosome map by arranging the genes in a linear series according to their relative cross-over frequencies. In Drosophila the relative positions of several hundred genes have been mapped out in this way.

When a new character appears in Drosophila it can be quickly determined by breeding experiments to which
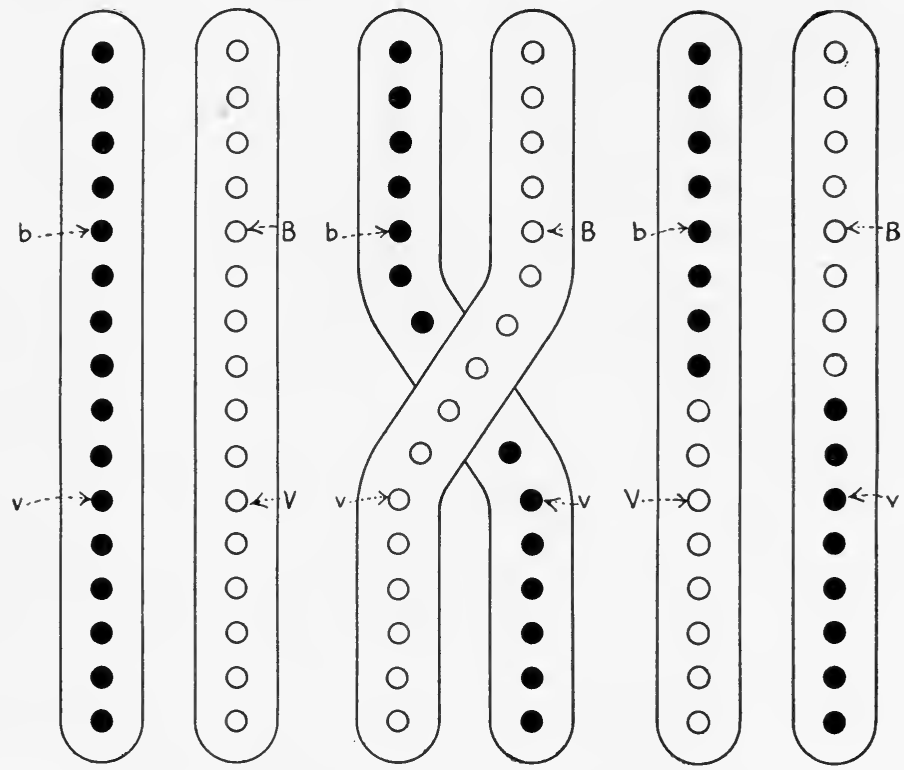

Fig. 32.-Diagram illustrating the mechanism of crossing over. In the fruit fly the characters of gray color and long wings are linked and presumably are carried by genes in the same chromosome. If gray $(B)$ and long $(V)$ enter the cross together from one parent and the corresponding recessive genes for black $(b)$ and vestigial wings $(v)$ enter from the other, and the chromosomes cross and break between the loci of these genes and then exchange segments, the one chromosome would contain the genes for black $(b)$ and long $(V)$ and the corresponding chromosome would contain the genes for gray $(B)$ and vestigial wings $(v)$. Usually cross-overs occur between these loci in about 17 per cent of cases.

linkage group it belongs. Then its frequency of crossing over with other characters of the group is worked out, and its relative position is assigned in the chromosome map. This daring attempt to explore the chromosome has had to justify its acceptance by meeting all possible objections. Geneticists are a very skeptical and critical lot, but none of them has succeeded in devising a rival theory of linkage 
and crossing over which has gained a considerable following. Not only do the numerous facts of normal heredity fit in with the requirements of the theory, but now and then the chromosomes behave in anomalous ways, and in many cases it has been possible to correlate these anomalies with equally irregular behavior in the transmission of visible characters. To illustrate, the geneticist finds that a group of characters normally belonging in the second linkage group suddenly becomes sex linked. He supposes that a part of the second chromosome has become broken off and attached to

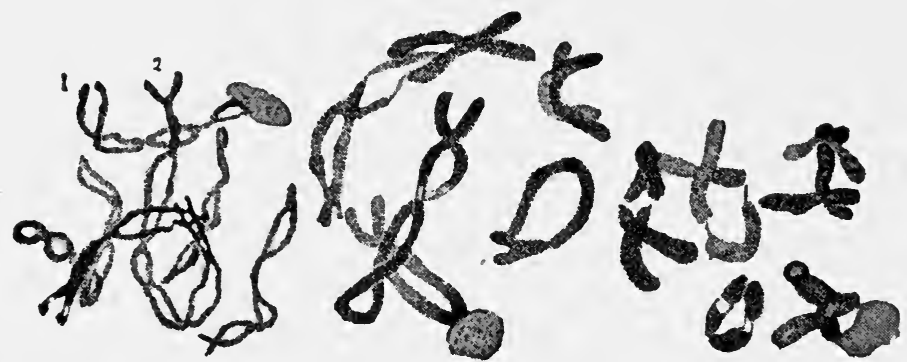

Fic. 33.-Paired chromosomes of Callisia showing chiasmas, or crosses between the threads. (After Sax.)

the $X$-chromosome, and he can tell about how big the piece is likely to be. Then he makes a preparation of the sex cells and finds upon microscopic examination that the $X$-chromosome has an extra piece attached to it, and that one of the second chromosomes is shorter than its mate. Predictions like this have been made and verified many times over in recent studies on the fruit fly. The so-called chromosome theory of heredity has come to be supported by so overwhelming a mass of evidence, and has been verified so many times by crucial test experiments, that practically all the hard-headed and skeptical geneticists the world over have no hesitation in accepting it.

Some day we might imagine the geneticist showing Mr. Smith a map of his heredity and saying: "Here in the four- 
teenth chromosome six units to the left of the attachment of the spindle fiber is the gene for that crooked little finger which you have probably derived from your paternal greatgrandfather. At this point in the twenty-first chromosome between your gene for red hair and the gene that predisposes you to bronchial asthma is the factor which causes you so much trouble from indigestion. In your fourth chromosome eight units from the left end (geneticists speak familiarly of the right and left ends of chromosomes) is a gene that would probably cause you to inherit your father's shortsightedness were it not prevented by its corresponding gene derived from your mother." Whether or not we shall ever be able to present such definite information for the edification of Mr. Smith, our illustration in no wise exaggerates the precision of the statements that can be made concerning the germ plasm of the fruit fly. While physicists have been exploring the atom, geneticists have been mapping out the chromosomes and completing a picture of a region that was an entire terra incognita to Darwin and Mendel.

Chromosomes have been mapped out in great detail in the common Drosophila melanogaster and less completely in other species of the genus. Less detailed maps have also been made of the chromosomes of Indian corn, the Jimson weed (Datura), the sweet and garden peas, and a few other plants and animals. In man little progress in studying linkage has been made because we cannot breed people at will and it takes many years to secure results. A serious drawback is encountered in the circumstance that each married couple does not produce several hundred children. Our knowledge of human heredity has to be picked up when and where opportunity offers. Everything that we know about it, however, fits into the general Mendelian scheme, and there is no ground for concluding that it differs in any essential respect from heredity in other organisms. 


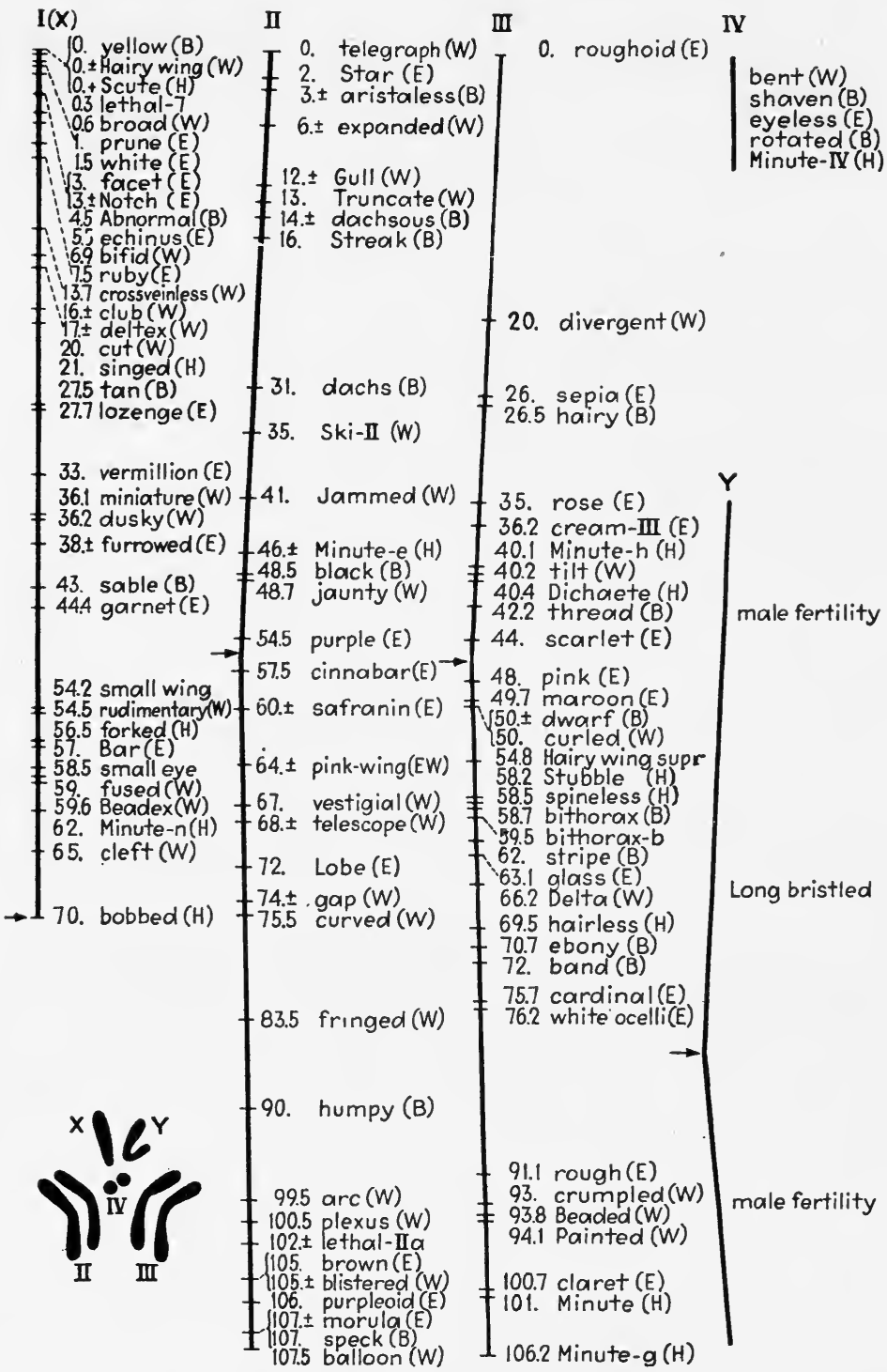

Fig. 34.-Chromosome map of Drosophila melanogaster indicating the positions of some but not nearly all the genes which have been located. The arrows designate the points where the spindle fibers are attached. The letters in parentheses indicate the part of the body in which the characters appear: $B$, body; $E$, eye; $H$, hairs; $W$, wings. The genes for fertility in the $Y$-chromosome have not been definitely located. (From Sharp adapted from Morgan, Sturtevant, Bridges and Stern.) 


\section{HUMAN GENETICS AND ITS SOCIAL IMPORT}

The recent studies of the structure of the giant chromosomes of the larvae of Drosophila, which we have alluded to in Chap. I, have enabled geneticists to associate many genetic factors with particular bands in the chromosomes. By studying many cases of breaks and reattachments of chromosomes, together with their correlated genetic phenomena, it is found possible to locate quite accurately the actual position that particular genes occupy in the chromosomes. One cannot identify the genes with any visible features of chromosome structure. We do not know whether the genes are in the stained bands or in the unstained material. But it can be stated that a given gene is very close to a particular band. This is as far as the genes have been hunted down at the present time. Certain genes have been shown to be a little closer together here or a little farther apart there than was formerly supposed, but the conclusions as to the linear order of the genes have only been more fully confirmed by this recent work.

\section{Suggested Readings}

Lindsey ('32), chap. 9. Morgan ('28), chaps. I, 2. Newman ('32), chap. 23. See also references for chap. 3 .

\section{Questions}

I. The characters of an animal fall into eight linkage groups. What would you infer as to its number of chromosomes?

2. In Drosophila black color and vestigial wings when they enter a cross together come out together in $8 \mathrm{I} .5$ per cent of cases; similarly vestigial wings and cinnabar tend to be associated in 90.5 per cent of cases. What may be inferred as to the distances separating the genes for these characters in the chromosome?

3. $A$ is linked with $B$ in 90 per cent of cases and with $C$ in 85 per cent of cases. $B$ is associated with $C$ in 95 per cent of cases. What are the relative positions of the genes for $A, B$ and $C$ in the chromosome?

4. If $A$ was associated with $B$ in 90 per cent and with $C$ in 85 per cent of cases as before and if $B$ was associated with $C$ in 80 per cent of cases, what conclusion would you draw as to the relative positions of their genes? 
5. Suppose $A, B$, and $C$ occur as in question 4 and a new character $D$ was found to cross over with $B$ in 5 per cent and with $C$ in Io per cent of cases, how frequently might it be expected to cross over with $A$ ? What would be its position in the chromosome with respect to $A, B$ and $C$ ?

6. If half of the characters normally associated with the fourth chromosome in Drosophila should suddenly become associated with characters whose genes are carried in the second chromosome, what happening might cause the change?

7. If a character were closely linked to a lethal gene, how would its prevalence be affected?

8. Why is it more difficult to study linkage in mammals than in the fruit fly?

9. To how great an extent is the doctrine of the individuality of the chromosomes modified in the theory of crossing over?

I0. If a Drosophila with linked characters $A B C D E$ in the order mentioned were crossed with $a b c d e$ and the $F_{1}$ crossed back to the recessive type $a b c d e$, producing some flies with the combination $A B c d E$ and others with the combination $a b C D e$, how would you explain the result? 


\section{CHAPTER VII}

\section{VARIABILITY - ITS KINDS AND ITS CAUSES}

VARIATION and heredity are commonly used as antithetic terms. The study of each of these phenomena has thrown much light upon the other. What we are chiefly interested in concerning heredity is how the characters that distinguish one individual or variety from another are transmitted. If all members of a species had exactly the same hereditary traits the problem of why offspring are such faithful copies of their parents would present a fascinating topic for speculation, but we would probably be able to contribute little toward its solution. It is because organisms vary and we are able to study the way in which their variations can be combined and separated that we have been able to discover laws of heredity, and to interpret these laws in terms of chromosome behavior. It is owing to the fact that garden peas present well-defined hereditary variations that Mendel discovered his fundamental laws of heredity. And it is only through the study of linkage and crossing over that it is possible to determine the location of particular hereditary factors in individual chromosomes. In short, most of our understanding of heredity is rendered possible only through the study of variations.

Variations fall into a number of classes. The most fundamental distinction is that between (I) somatic variations, or those arising in the body as a result of its own activities or the influence of the environment, and (2) germinal variations, which depend upon changes in the germ plasm. If one develops his muscles through exercise, or acquires a coat of 
tan through exposure to the sun, such variations are purely somatic, and in the opinion of most geneticists they have no influence upon the germ cells and hence no effect upon subsequent generations. Whether or not somatic variations, or acquired characters, play any part in evolution depends upon whether or not they can affect the germ cells in such a way as to cause similar variations to appear in the offspring. This, as we have seen, is at least a questionable possibility.

There is no doubt whatsoever that variations which originate in the germ plasm are capable of hereditary transmission. To a considerable extent the new variations which arise are the result of varied combinations of genes and represent simply the expression of Mendelian segregation. When a black sheep turns up in a flock that has produced nothing but white sheep for several generations, the new type is probably the product of the meeting of two recessive genes for which both parents were heterozygous. Blackness in sheep is known to be a recessive character and hence when black sheep are bred together nothing but black progeny is produced. When a purple pea is formed by crossing two whites and when the purple hybrid produces a variety of other colors, whites among the rest, the striking display of variability is really nothing but the result of different combinations of old factors. What superficially appear as exceptions to the laws of heredity are really manifestations of the way in which heredity works. Such variations are analogous to the different kinds of hands one may obtain by shuffling and dealing a deck of cards. They represent only new combinations of old things.

From time to time germinal variations of another kind make their appearance. There are many cases in which the normal number of chromosomes is doubled, giving rise to four sets of chromosomes instead of two. Occasionally there are three sets of chromosomes, or rarely only one. Sometimes 
the abnormal duplication of chromosomes affects only one or two pairs; or, again, a single chromosome or rarely more may be lost. All such changes in chromosome number affect the visible characters of the organism to a greater or less extent. Sometimes parts of a chromosome are lost or duplicated, or a part may be broken off from one chromosome and stuck on to another. Such translocations are common in the progeny of plants and animals treated by radium or $X$-rays. Numerous chromosome anomalies have been described, especially in Drosophila, and the peculiar genetic phenomena that followed have been explained as a result of the exceptional chromosome behavior.

The germinal variations of most fundamental significance are those occurring in the constitution of the genes themselves. These are called gene mutations. Through the study of linkage, it is now possible to locate quite precisely the region of a chromosome in which a new gene mutation occurs. In a given locus in the sex chromosome of Drosophila there have been twelve different mutations described, each causing a characteristic change in the color of the eyes.

The term mutation as commonly used in the literature on genetics connotes a sudden discrete mutation which breeds true. Formerly more than now, the term was applied mainly to extensive departures from the normal type, such as gave rise to hornless breeds of cattle, the navel orange, or strains of albino mice. Such stable and discrete germinal variations were found to range in magnitude from monstrosities to very small changes which are barely detectable. There may be no essential difference between a germinal variation, which can be observed in only one spot on a butterfly's wing, and one that causes a widely divergent variety. Even a single gene mutation may produce so profound a disturbance of normal development as to cause the death of the organism. 
Both gene mutations and those due to chromosome anomalies may play an important part in the origination of new varieties and in the general course of organic evolution. The first process is doubtless the more important and fundamental. For the most part both kinds of variations appear to arise spontaneously without apparent cause. They just happen. But why? The problem of the causes of variations has long been of interest to the evolutionist. The Lamarckian had at least a partial answer to the problem in that he could account for variations as due to the transmission of acquired characters. Many variations as we have seen are due to Mendelian segregation. The other types of germinal variations we have discussed are due to whatever causes the anomalous behavior of chromosomes and the alteration of individual genes. But however variations may be caused, a good deal of insight has been gained into the real nature of variability, because we can now distinguish the different classes of variations in a way that was impossible a generation ago.

This insight does not in itself give us a plausible explanation of variability, but it is helpful in giving a clear understanding of just what is to be explained. Gene mutations are frequently considered as due to some alteration in the chemical constitution of the gene. A gene is a very small body not very many times larger than some of the larger molecules of protein. Some geneticists have held that a gene consists of a single organic molecule. One might interpret gene mutation as caused by the addition or loss of an atom or radical, or possibly as due to some internal rearrangement of atomic groupings. From this viewpoint it might be thought possible to induce gene mutations by agencies which might exercise some chemical effect upon the germ plasm. Accordingly experiments have been tried by introducing substances into the body and testing their possible effect upon the offspring. 
Fruit flies have been exposed to alcohol, ether, methylene blue, and other chemicals, but out of many thousand progeny of the treated individuals, no more mutants appeared than among the progeny of untreated flies bred as controls. Several other efforts to induce mutation by means of chemicals have yielded negative results. Apparently, the germ plasm possesses a high degree of stability. It is doubtless for this reason that organisms have been able to perpetuate their kind over long periods of time with very little change.

There have been some experiments reported in which it has been claimed that heritable modifications have been induced by chemical means. One of the most noteworthy of these was performed by Dr. C. R. Stockard upon guinea pigs exposed to the influence of alcohol. With commendable caution the animals employed were first bred and found to produce normal offspring. They were then exposed to the fumes of alcohol about five or six times a week for a period of several months. In each trial they were made to inhale the fumes of alcohol until they manifested signs of incipient intoxication. After this preparation for parenthood they were bred. Among the descendants of the first three generations there were several weak and defective offspring. There were smaller litters than in the animals bred as controls and more of the young died soon after birth. In a later communication Stockard reports that the fourth generation of the alcoholized guinea pigs were almost free from defects and apparently rather more vigorous than the controls. This result he attributed to the elimination of the defective germ plasm which may have been caused by the poisonous influence of alcohol. If alcohol was really the cause of the inherited defects observed, these experiments may have an important bearing on the origin of hereditary defects in man. Human beings have long indulged in alcoholic beverages to a degree that has been productive of many physical and mental disabilities. It 
has been conjectured that alcohol may injure the germ plasm and thus be a cause of mental defect and physical disorders of all sorts. For some reason new strains of hereditary defectives arise from time to time, and it is perhaps natural that their origin should be attributed to alcohol or other "racial poisons." But it should also be borne in mind that defective mutations arise from time to time in almost all kinds of organisms. For this reason one should subject the evidence that alcohol is the cause of hereditary defects to a very careful scrutiny. The influence of alcohol on the birth weight of white rats has been studied by MacDowell and by Hanson and Heys, but they arrived at different results. MacDowell has found that the descendants of alcoholized rats learn a maze rather more slowly than the descendants of their litter mates which were not given alcohol. The possibility is not entirely excluded that the parents of the treated and the control strains may have been genetically different from the start, a possibility by no means excluded by the fact that they belonged to the same litter. Dr. Agnes Bluhm has made extensive investigations on the effect of alcohol upon fertility, weight at birth and longevity of the descendants of white mice, and has concluded that alcohol has an unfavorable effect upon the progeny which in some respects continued as far as the seventh generation.

Pearl, on the other hand, reports that the descendants of alcoholized fowl were on the whole superior to those of untreated individuals. The number of fertile eggs produced by the alcoholized strain was less than in the controls, and the conclusion was drawn that alcohol causes the weaker germ cells to be eliminated. It has been suggested that Stockard's and Pearl's results are not necessarily opposed, since alcohol might cause germ cells to become injured as well as effecting the elimination of cells less able to withstand its influence. 


\section{HUMAN GENETICS AND ITS SOCIAL IMPORT}

F. M. Durham has repeated Stockard's experiments on guinea pigs, but she exposed the animals to a longer period of alcoholization. No defects were observed in the offspring of the treated animals, although a larger number were studied than in the experiments of Stockard. Durham is inclined to believe that the defects observed in Stockard's guinea pigs, in so far as they were hereditary, were due to the accidental outcropping of recessive genes.

Dr. M. F. Guyer has attempted to induce germinal variations by a different method. Making a preparation of the ground-up lenses of the eyes of rabbits, he injected this material into the blood of a fowl. After a number of treatments the blood of the fowl, which had presumably developed an antibody to lens substance, was injected into pregnant rabbits. Of the sixty-one rabbits which were born to the treated mothers in one set of experiments, four had wellmarked defects in one or both eyes, and the eyes of five others were more or less abnormal. One of the most common defects observed was opaque lenses. Defects of the eye were obtained in four separate and presumably unrelated strains of treated animals. The defect once started was found to be hereditary. Apparently it behaves as a partially recessive trait. Defective-eyed rabbits mated with normal ones usually have normal progeny, but the mating of two defective-eyed rabbits produced mostly defective-eyed progeny, although occasionally a normal individual would arise.

The objection that, possibly, eye defects may have happened to turn up in the progeny of the treated female, Dr. Guyer has endeavored to meet by repeating the experiment with different lines and by breeding a considerable number of control animals. Only one case of defective eyes was observed in more than 2,000 controls and Dr. Guyer remarks that "Whatever the final explanation of just what has taken place in the germ plasm may be, it seems reasonably sure 
that the results are in some way the outcome of the experimental treatment."

These experiments have been interpreted by some Lamarckians as supporting the theory of the transmission of acquired characters. Dr. Guyer himself does not put this interpretation on his results. He suggests as an alternative possibility that "the eye and the germ of the fetus are influenced separately by the antibodies which have entered from the mother's blood by way of the placenta."

Guyer's experiments aroused widespread interest and led others to attempt to duplicate their results. J. S. Huxley and A. M. Carr-Saunders have repeated Guyer's experiments without obtaining any defective-eyed progeny from the treated animals. The spontaneous appearance of defective eyes in normal rabbits has been recorded in several instances and seems not to be so rare an event as Guyer supposed. The experiments of Ibsen and Bushnell and later Ibsen, who endeavored to duplicate Guyer's results, failed to yield confirmatory evidence. Out of fifty-nine offspring of treated female rabbits only one showed a slight defect, but a slight eye defect was noticed also in the offspring of rabbits that had not been treated. Of I 70 offspring of rabbits whose lenses had been destroyed by a needle (a procedure which according to Guyer results occasionally in defective-eyed progeny) all were normal eyed as were all the grandchildren. On the other hand, "six markedly defective-eyed animals have been found among the 920 having an untreated ancestry." Three of these occurred in one family. In a later communication reporting the results of further experiments there were no eye defects in 177 rabbits of the first generation produced from treated parents, nor in $\mathrm{I}_{4} 8$ of the second generation. The only defective-eyed animals occurred in a family of three produced by an $F_{2}$ female and an $F_{1}$ male, but there was evidence that the defects in these individuals were caused by an infection. 


\section{HUMAN GENETICS AND ITS SOCIAL IMPORT}

There are several sources of error in experiments designed to induce variations. One of the chief of these is the uncertainty as to whether the animals chosen for experimental treatment are genetically different from those used for controls. If one essayed to test the efficacy of an experimental treatment which was alleged to cause the production of black sheep, it might well happen that some of the treated animals were heterozygous for blackness, while all the control animals were free from this defect. This might happen of course several times over. It seems not unlikely that a good deal of the discrepant results of experiments designed to test the hereditary influence of alcohol and other substances may be due to differences in the genetic make-up of the animals chosen for the experiment. From the a priori standpoint the production of variations by the administration of chemical substances seems probable enough, but on the whole the evidence for the efficacy of most so-called "racial poisons" leaves much to be desired.

In the effort to produce variations by artificial means a number of investigators have turned to the employment of $X$-rays and radium. Both these agencies are able to produce chemical effects within the body. Severe dosage of $X$-rays or radium has been shown to injure or even to kill the chromatin of irradiated germ cells, and it seems a probable inference that changes induced in the chromatin might prove to be a source of hereditary variability. Bagg and Little subjected an inbred strain of mice to $X$-rays and found that in the second and subsequent generations the progeny exhibited a number of variations such as club feet, defective eyes, absence of one or both kidneys and other anomalies. Similar results were later reported by Bagg in the progeny of an unrelated strain of mice subjected to $X$-rays.

The most extensive and indubitable results of treatment with $X$-rays were reported by $\mathrm{H}$. J. Muller, who found 
that the percentage of mutations appearing in the progeny of irradiated fruit flies was increased to a startling degree. Many of the gene changes so produced proved to be lethal, but in addition to these, mutations of all sorts appeared in great profusion. The mutation rate was speeded up about I 50 times as compared with that of the controls. This work was soon confirmed by many other workers and is now a common method of inducing mutations on a large scale.

Radium also has been shown to be a fertile source of mutations in both animals and plants, and some rather striking results have been reported in causing mutation in Drosophila by exposure to a nearly fatal degree of heat. Unquestionably environmental factors are able to modify the germ plasm so as to cause stable hereditary variations. From the standpoint of adaptation most of these variations are either unimportant or positively injurious. We are still ignorant as to what causes the new strains of hereditary defectives that are continually arising in our human stock. The lame, the halt, and the blind keep turning up, and while many of these defects tend to be weeded out and disappear, there is something which continues to keep up the supply. Is it alcohol, disease, bad environment, or as has been suggested, the radiations which emanate from the earth? It is not unreasonable to suppose that untoward agencies may result in hereditary defects, but it should be remembered that defective mutations arise in all sorts of organisms quite irrespective apparently of the conditions under which they live.

\section{Suggested Readings}

Guyer ('27), chap. 17. Jennings ('30), chaps. I4, 15. Wells, Huxley, Wells, book 4 , chap. 7 .

\section{Questions}

I. If a recessive gene should arise, when and under what conditions would it become manifest? 
2. Which are the more common, dominant or recessive mutations?

3. Is the dark color of tropical races due to the effect of sunlight in successive generations? Does environment tend to produce a similar color in Swedes and Lapps living for generations in a similar climate?

4. If size is determined by the influence of endocrine glands, can it be due to genetic factors?

5. What is the essential difference between the production of a heritable germinal variation by environmental means and the transmission of an acquired character in the Lamarckian sense?

6. What device was employed by the thrifty Jacob to produce the desired variations in cattle? See Genesis, Chap. 30, 32-43.

7. Which would you expect to be the more variable, self-fertilized or cross-fertilized plants?

8. Do you think Darwin was right in attributing the variations of cultivated plants and domestic animals largely to the influence of changed conditions of life? What alternative explanation might be offered?

9. Compare the influence of alcohol on the race due to $(a)$ its possible effect in causing hereditary defects, and (b) its effect as a selective agent in eliminating unfavorable variations that might arise.

ro. Why did Burbank make numerous crosses in his efforts to produce valuable varieties of plants?

II. A breeder crosses a black female and an albino male rabbit and obtains some black and some albino offspring. He subsequently breeds the black rabbit to another black and obtains a few albinos along with blacks. He attributes the albinos in the second mating to the influence of the previous albino male parent. What other explanation for the albino offspring can you offer? Do you think that the influence of a previous male parent (telegony) is probable in the light of present knowledge of the physical basis of heredity? 
CHAPTER VIII

\section{THE MEASUREMENT OF VARIATION}

ANY variations such as the heights of human beings 11 form a regularly graded series between the two extremes. Such variations are called fluctuations or sometimes modifications, and for the most part they represent merely somatic changes and are not hereditary, at least to any recognizable extent. Germinal variations may occur among them, but they are not easily distinguished from those which are environmentally caused. When enough cases are collected the phenomena of variability often exhibit statistical uniformities which it is desirable to measure. The Belgian astronomer, meteorologist, and anthropologist, Quetelet, was greatly impressed by the fact that when human beings are studied in the gross their peculiarities exhibit a uniform character in striking contrast to the capriciousness of individual behavior. By tabulating the results of numerous measurements of the heights of soldiers he found that these distributed themselves about the mean, or average, in fairly close agreement with the mathematical laws of probability.

The statistical study of variation initiated by Quetelet was carried further by Francis Galton who employed it extensively in his valuable pioneer investigations in genetics. The mathematical treatment of biometric problems has been ably developed by Karl Pearson, the director of the Laboratory for National Eugenics founded by Galton in University College, London. A large part of the formulas employed by workers in biometry are the products of Pearson's investigations. 
Variations which fluctuate about the mean value are often represented graphically in the form of a so-called frequency curve, or frequency polygon. Suppose that we wish to make a curve of the variability in the heights of a thousand men. We first lay off on a base line a number of points corresponding to the various classes of height into which we divide our group, beginning at the left with the class including the shortest individuals. If our group is divided into classes differing by one inch in height, we should erect over the point in our base line corresponding

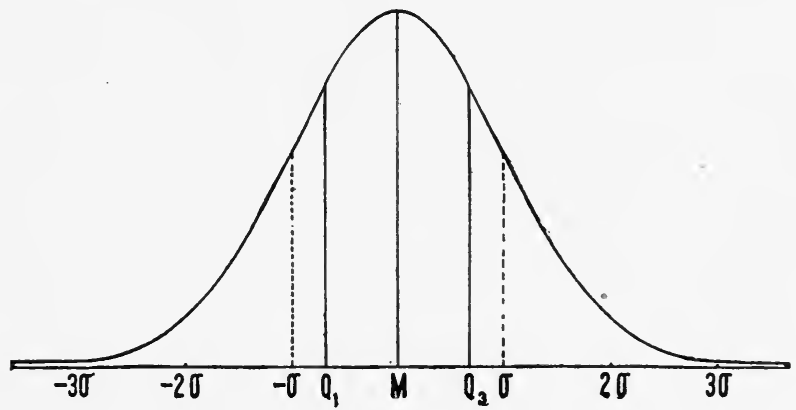

Fig. 35.-The normal frequency curve. $M$, mean. The lines over $\mathscr{Q}_{1}$ and $\mathscr{Q}_{3}$ divide the areas of the curve on either side of the mean into two equal parts. The dotted perpendiculars over $\sigma$ and $-\sigma$ are separated from the mean by a distance equal to the standard deviation. The distance between $\sigma$ and $-\sigma$ includes 68.3 per cent of the area of the curve. 95.5 per cent of the area lies between $2 \sigma$ and $-2 \sigma$ and 99.7 per cent lies between $3 \sigma$ and $-3 \sigma$. (After Babcock and Clausen.)

to the shortest individuals a perpendicular whose length is in proportion to the number in that class. Then we do the same with each class successively and finally draw a line over the tops of the several perpendiculars. The perpendicular corresponding to the most numerous class, which in this case would be near the center of our figure, would represent the mode. The perpendicular representing the mean, or average height, would divide the frequency polygon into equal areas and would lie quite close to the mode. The formula for computing the mean is $M=\Sigma f V / n$, $\Sigma$ meaning sum, and $f V$ the frequency of the variants, and 
$n$ the number. This means simply that to get the mean height of a number of human beings we multiply the various heights by the number of individuals of the height in question, add them all together, and divide by the total number in the group. In most frequency curves the mean is not far from the mode, or, in other words, average individuals usually constitute the most numerous class as is notoriously

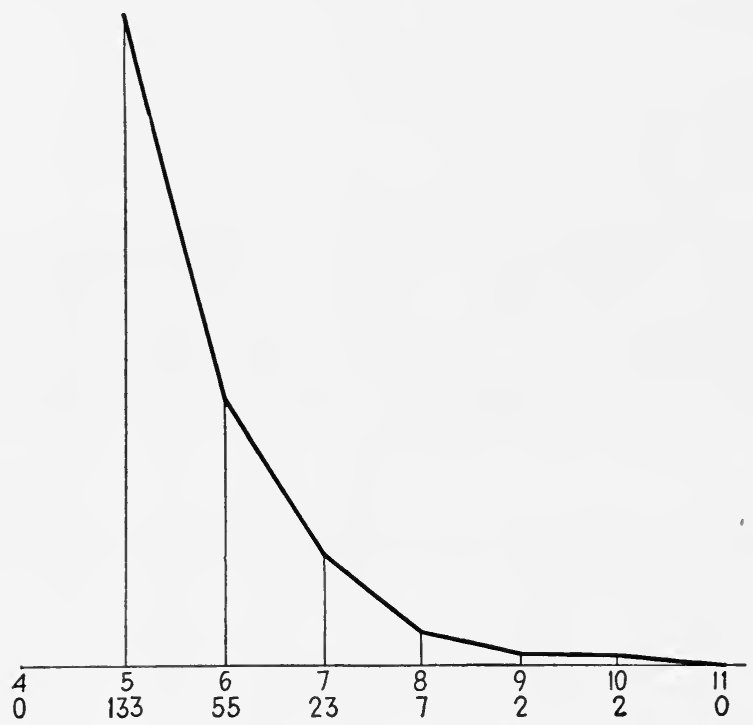

Fig. 36.-Curve of variability of the buttercup. The mode is at one extreme, there being no flowers with less than five petals.

the case with the height of human beings. Commonly also the curve is approximately symmetrical on either side of the mode. Sometimes the curves are asymmetrical, or skew, and the mode may be even at one extreme. DeVries found, in counting the petals of buttercups, that there were 133 with five petals, none with a less number, 55 with six, and $23,7,2$, and 2 with seven, eight, nine and ten petals respectively. The variability of this form would be represented graphically by a one-sided curve with its mode at one extreme. 
Occasionally frequency curves have two or more modes. Should we tabulate the sizes of individuals in which the two sexes differ markedly we should obtain a double humped curve with one mode for females and another for males. An interesting illustration of the probable significance of a bimodal curve is furnished by plotting the variability of the head lengths of the spermatozoa of certain insects. It was found by Zeleny and Senay that when the lengths of the sperm heads were measured and plotted they gave a frequency polygon such as is shown in Fig. 37. The probable explanation is that the spermatozoa fall into two classes, the one containing the $X$-chromosome having larger heads

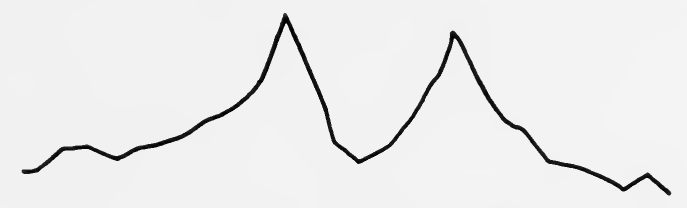

Fig. 37. - Bimodal curve based on measurements of the lengths of 500 sperm heads from a single male of Corizus lateralus. (After Zeleny and Senay.)

than the other in which this chromosome is absent. The bimodality is indicative of the dimorphism of the population of spermatozoa.

The shape of frequency curves tells a number of things about the range, average amount, and distribution of variability within a given species or group. If the curve has a wide spread, it indicates that the group is highly variable. If it is very narrow, the individuals clustering closely about the mean, the variation within the group is manifestly very slight. It is often desirable to obtain a definite measure of the amount of variability in a group in order to compare it with the variability of some other group. The eminent zoologist, W. K. Brooks once maintained that males are more variable than females, and that one of the important functions of the male sex is to furnish the vari- 
ability which is an essential prerequisite for evolution. If we wish to ascertain whether men are more variable than women, we would not be able to do so by simply watching the crowds which pass along the street. Evidently we should have some fairly precise method of measuring the degree of variability in order to solve such a problem. With the biometric methods devised by Pearson a number of workers began to measure the relative variability of the two sexes in several species of animals. If the males are really deserving of the proud position which Brooks has accorded them, the facts should be readily demonstrable by the use of a little biometry. When the relative variability of the sexes was subjected to accurate measurement, it turned out as a rule that they were about equally variable, although in some species the greater variability was exhibited by the females.

The first step in obtaining a measure of variability is to ascertain the extent to which each individual deviates from the mean of the group. We can find the average deviation from the mean by multiplying the figure expressing the extent of deviation of each class by the number of individuals in each class, adding all the products together and then dividing by the total number. The formula for the average deviation is A.D. $=\Sigma f D / n$. The measure of variability most commonly employed, however, is the standard deviation. In deriving this each deviation is squared, the squared deviations are then added and divided by the total number, and the square root of the quotient represents the number sought. S.D. $=\sqrt{\frac{\Sigma f D^{2}}{n}} \cdot$ Frequently the standard deviation is represented by the Greek letter $\sigma$.

It is evident that the standard deviation is expressed in terms of the units of measurement employed. The standard deviation for the height of men is a certain number of inches 
or fractions thereof, and the standard deviation for weight is stated in terms of pounds or other units of weight. If we are dealing with variations in different kinds of organisms it is necessary to reduce them to a common standard. The standard deviation of the weight of a lot of elephants would doubtless be many pounds, but a group of mice could not possibly include variations of that magnitude. If we wish to compare the variability of elephants and mice as to weight, we would have to consider the variability of each in relation to the mean of its own group. For this purpose use is made of the coefficient of variability, which is obtained by multiplying the standard deviation by 100 and dividing it by the mean. This gives the relative variability in terms of the per cent of the mean, the formula being C.V. $=(1 \circ 0 \times \sigma) / M$.

Expressions for means, standard deviations, and other measures may have very different degrees of reliability owing to the different numbers of cases upon which they are based. If I should make assertions as to the relative stature of Norwegians and Italians based on averages of four individuals of one group and five of the other, I might be led into serious error, because I might happen to get hold of unusually tall Italians and very short Norwegians. Evidently the larger the number of individuals measured, the more reliable the averages are likely to be. What is called a probable error is, as its name implies, a measure of the extent to which a figure is likely to be wrong. All the expressions we have discussed are apt to be a little wrong on account of the fact that they are based on a limited number of cases. The probable error is taken to be a value such that a given instance is as apt to fall within as without that value. If a thousand people should all endeavor to get a very exact measure of the height of a given individual, most of their measurements would doubtless differ by a slight amount. The differences would probably fall into a 
fairly regular curve, symmetrically distributed about the true value. In what statisticians call the normal curve the lines dividing each half into equal areas are called quartiles and the distances of these quartiles from the means would represent the probable error of a given measurement. This is a definite fraction, 0.6745 of the standard deviation, provided our curve is what is called the normal curve of error. The formulas for the probable errors of the mean, standard deviation, and coefficient of variation are as follows:

$$
\begin{aligned}
\text { P.E.M } & =0.6745 \frac{\sigma}{\sqrt{n}} \\
\text { P.E. } & =0.6745 \frac{\sigma}{\sqrt{2 n}} \\
\text { P.E.c.v. } & =0.6745 \frac{\text { C.V. }}{\sqrt{2 n}}
\end{aligned}
$$

The probable error of the difference of two quantities is 0.6745 times the square root of the sum of the squares of the sigmas of these quantities.

$$
\text { P.E. } ._{\text {diff. }}=0.6745 \sqrt{\sigma_{x}^{2}+\sigma_{y}{ }^{2}}
$$

If a difference is more than three times its probable error, it is probable significant, i.e., not due to chance.

Besides studying the variations of individual traits, it is often desirable to investigate the extent to which one kind of variation is tied up with another. We might be curious to know the extent to which the weight of human beings is related to their height. It is a matter of common knowledge that short people are generally lighter in weight than tall people, although there are occasional exceptions. We might also like to know how breadth between the shoulders is related to height or to weight. Or again we might desire to find out how infant mortality varies in relation to the wages of the father, or the amount of schooling of the 


\section{HUMAN GENETICS AND ITS SOCIAL IMPORT}

mother. Where things tend to vary together, we suspect that there. is some causal connection between them, or that both may be influenced by a common cause. The mathematical expression which indicates the extent to which two things vary together is called a coefficient of correlation. It is always expressed as some fraction of $I$ and it may range from $\circ$ to $\mathrm{II}_{\mathrm{I}}$ or $-\mathrm{I}$. If, for instance, people tend to have large families in proportion as they are lacking in money, we would say that there is a negative correlation between wealth and family size, as in fact there seems to be.

Coefficients of correlation are extensively employed not only in genetics and in other departments of biology, but in psychology, education, economics, and the social sciences in general. The first to make use of coefficients of correlation in the study of heredity was Francis Galton, who was the first also to work out a mathematical formula for their computation. The method was perfected by Karl Pearson to whom we owe the formulas now most frequently employed. The so-called biometric school of Galton, Pearson, and their coworkers has made extensive studies on the correlation between parents and offspring and between siblings for a number of hereditary traits. Many of the correlations were found to center around 0.5 . In longevity, which shows a tendency to run in families, the correlation is much weaker owing to the occurrence of many deaths in infancy and childhood, and the influence of mortality arising from purely fortuitous causes. It is often assumed that a parent-offspring correlation affords a measure of what is called the "strength of heredity." What it really measures, as commonly employed, is the degree to which the offspring and one of its parents tend to vary together in relation to the mean of their groups. It is inferred that this correlation is due to their common inheritance. When we are dealing with such characters as eye color, which is not sensibly affected by environ- 
mental factors, this inference is justified. If, however, we were studying the possible heredity for stoop shoulders and dealt with a population in which it was customary for fathers and sons to follow the trade of shoemaking, we might be in doubt as to whether the correlation between fathers and sons for this character was due to heredity or their traditional occupation. The fact that fathers and sons are both longlived may be due in part to the fact that both live in a measure under conditions which in some families tend to prolong life and in others tend to shorten it. A parentoffspring or a fraternal correlation, therefore, may at times be due to causes other than heredity, and we have to interpret the correlation in the light of the possible factors which may bear upon the problem.

Another circumstance which should be considered in interpreting the significance of correlation is that degrees of correlation are determined not only by similarities, but also by dissimilarities. A correlation is a measure of covariance, and where there is no variation there is no correlation. This fact has been brought out by the studies of heredity in pure lines of beans carried on by the Danish geneticist Prof. Johannsen. The beans with which Johannsen worked are normally self-fertilizing and were found to present no hereditary variability. A pure line consists exclusively of homozygous individuals, and hence all the variability it contains is of a somatic noninherited kind. The variations of one individual are no more apt to be like those of its parent than those of any other member of the group. Hence there being no tendency for parent and offspring to vary together there is no correlation.

I might study parent-offspring correlations in a dozen different pure lines and obtain a negative result in all of them, although each line might be genetically different from all of the others. If now I mix them all up so that within 


\section{HUMAN GENETICS AND ITS SOCIAL IMPORT}

my group I get a considerable amount of genetic variability, I would get a certain degree of correlation because parents and offspring would tend to depart from the general mean in the same way. As a matter of fact, parents and offspring tend to resemble each other more closely in a pure line than in a heterozygous and diversified population. Nevertheless, the parent-offspring correlation would be $\circ$ in the pure line and anywhere from $O$ to $I$ in the mixed group.

From the nature of the case, correlations are based upon somatic resemblances. There is no resemblance in color between a purple sweet pea and the two white parents from which it might arise, nor is there much resemblance between the purple $F_{1}$ and its variously colored progeny. But all these striking differences in color are quite as much the result of heredity as the close similarities within a strain of white peas. In such a case, the study of heredity by working out correlations for color would be of little value. In general, the methods of analysis followed by the Mendelians have proved much more fruitful than the statistical studies on heredity made by the biometricians. This is no reflection upon the value of biometric methods. They have their legitimate and very useful place in numerous problems of genetics and even in Mendelian analysis, but we should recognize their limitations and know when and where to apply them.

\section{Suggested Readings}

Lindsey ('32), chap. I1. Walter ('30), chaps. 2, 3. Babcock and Clausen ('27), chap. I7.

\section{Questions}

I. A plant produces 2 flowers with 8 petals, 6 with 9, 17 with 10, 30 with II, 50 with 12,32 with I3, I9 with 14,7 with 15,3 with 16 and I with 17 petals. Construct a frequency curve from these data, and calculate the mean, standard deviation, and coefficient of variability. 
2. How much does the mode in this curve differ from the mean? How much does it differ from the mean in Fig. 36 ?

3. What is the probable error of the mean in question I ?

4. Why is the standard deviation of the $F_{2}$ of a cross usually greater than that of the $F_{1}$ ?

5. Why is the variability of the $F_{1}$ usually no greater than that of the parental types?

6. Among the progeny of the $F_{2}$ would you expect to find some lines presenting much more variability than others? Illustrate and explain.

7. What would be stated by saying that there is a negative correlation of $-\mathrm{I}$ between fertility and years of schooling?

8. Why is a coefficient of correlation between parents and offspring not an adequate measure of the "strength of heredity"?

9. Suppose that a character is not influenced by the environment. Why would the correlation between parents and offspring for this character probably be considerably less than I ?

Io. What is the effect of a high degree of somatic variability upon parent-offspring coefficients of correlation?

II. How might a parent-offspring correlation be brought about in a pure line?

12. How does the employment of measures of variability afford support for the multiple factor theory of so-called blending inheritance? 


\section{HEREDITY IN MAN}

7 HE immense amount of research in genetics that 1 followed and was stimulated by the rediscovery of Mendel's law of heredity in I900 has abundantly demonstrated the wide applicability of the principles that Mendel - so thoroughly established for his garden peas. That all sorts of animals and all sorts of plants from the highest down to and probably including many one-celled organisms should transmit their hereditary qualities in precisely the same manner is a very remarkable fact. The investigations of the cytologists have shown the reason for this in the striking similarities of chromosome behavior in the reproductive processes of plants and animals. In the light of such facts, it is scarcely credible that man, whose differences from the anthropoid apes entitle him at most to membership in a distinct family, should differ from other organisms in the way in which his hereditary traits are transmitted.

Inevitably the rediscovery of Mendel's law led to a reawakening of interest in human heredity. One of the first traits studied from the Mendelian standpoint was eye color. It had been known especially through the studies of Galton that the inheritance of eye color is often alternative, and that matings of two brown-eyed individuals or a browneyed and a blue-eyed individual may produce children whose eyes are brown, blue, gray, or even the pink color characteristic of albinos. As a rule blue-eyed parents produce nothing but blue-eyed children, a fact which indicates that blue is recessive to darker colors. The results of most matings 
are consistent with this interpretation. Eye color, however, is a highly variable character and is apparently influenced by modifying factors and variations in dominance. Occasionally a brown-eyed child is born to parents whose eyes would ordinarily be classed as blue, although it may be that one or both parents were heterozygous with very imperfect dominance of the gene for dark pigment. It would hardly be safe to decide a case of disputed parentage on the theory that two blue-eyed parents cannot have a dark-eyed child.

In the transmission of hair color, the darker shades tend to dominate over the lighter ones. One would not expect a dark-haired child from two flaxen-haired parents, but light-haired children are often born to dark-haired (presumably heterozygous) parents. Red color in hair seems to be transmitted independently of the dark melanin pigments, and it may be totally obscured if much melanin is present, or it may produce a chestnut hue if the dark pigment is less plentiful. Where there is little melanin, the redness may be very conspicuous. The factors causing the various shades of human hair are probably numerous, as is demonstrably the case with the inheritance of coat color in mammals. There are many instances in which the hair color in children is somewhat darker than that of either parent. Similar phenomena are commonly observed also in rabbits and other rodents.

Kinky or woolly hair which is a normal racial character in many tribes of Negroes is a partially dominant (or partially recessive) feature. Baldness is transmitted as a sex-limited trait, being dominant in man and fortunately recessive in women. In skin color, the darker shades are partially dominant over the lighter ones. All shades are dominant over albinism, which is commonly due to the complete absence of pigment. Albinos, whether they occur 
among human beings or the lower animals, have pink eyes, imperfect vision, and are relatively deficient in vitality. Although albino mutants have arisen thousands of times, and although albino races of rats, mice, and rabbits have been reared for many generations, no albino variety has ever succeeded in establishing itself in a state of nature. The curious condition known as partial albinism characterized by spots devoid of pigment in an otherwise dark skin behaves as a dominant character. The white forelock or flare, which often runs through several generations, is a variety of this trait.

The ordinary shades of skin color probably depend upon many factors. The mulattoes arising through crosses between Negroes and whites have an intermediate skin color, and their children show considerable variation indicative of the Mendelian segregation of color factors, but the number of such factors involved is uncertain. Stature, like skin color and size inheritance generally, is a multiple factor character. Children may be either taller or shorter than their parents, owing to the peculiar combinations of factors influencing growth. The number of such factors is very difficult to ascertain. In the exceptional case of achondroplastic dwarfs a single gene may be responsible for lack of growth, possibly as a result of some glandular disturbance which causes the bones to cease growing in length. These dwarfs usually have large heads and deformed bodies. The women are often incapable of giving rise to a normal birth, and recourse must be had to a Caesarean operation. In some cases the trait appears to be dominant and in others recessive, and sometimes it seems to occur independently of genetic factors. Dwarfs in whom the parts of the body occur in normal proportions are commonly not traceable to hereditary influences. Among the hereditary anomalies of growth, the condition known as split hand or 
lobster claw is one of the most unfortunate. The digits are fused together and disproportionately developed. The trait commonly behaves as a dominant. The afflicted

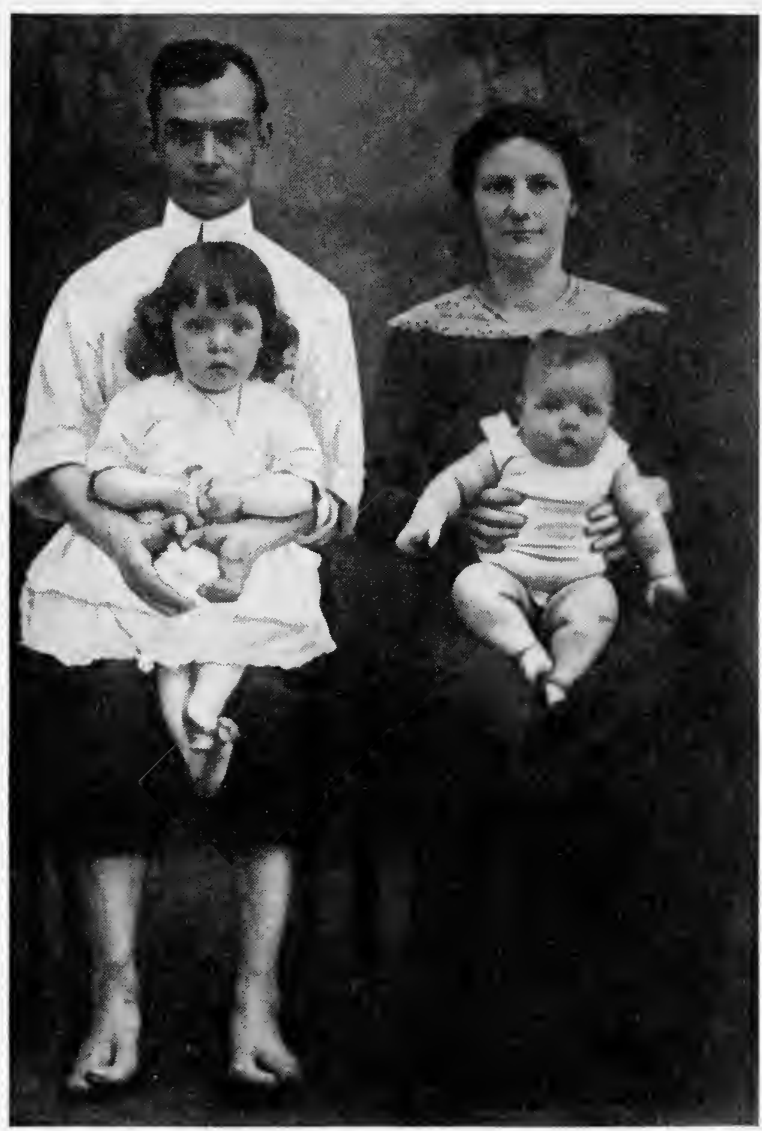

FIG. 38.-Family showing the inheritance of syndactylism, or lobster claw. The trait is shown in the father and in both children. A brother of the father was syndactylous and another brother had one supernumerary toe. The three sisters of the father were normal. (From Eugenical News.)

individuals are incapacitated for most employments. Nevertheless, there are several records of their marrying and transmitting their defect through several generations. Pearson has reported the case of a woman who bequeathed 


\section{IO2 HUMAN GENETICS AND ITS SOCIAL IMPORT}

this undesirable defect through four generations to thirtytwo descendants, a considerable proportion of whom have spent a large share of their lives in workhouses. An even more severe deformity, in this case consisting of the complete amputation of hands and feet, has been described in a family from Brazil. The mother was normal, but in the

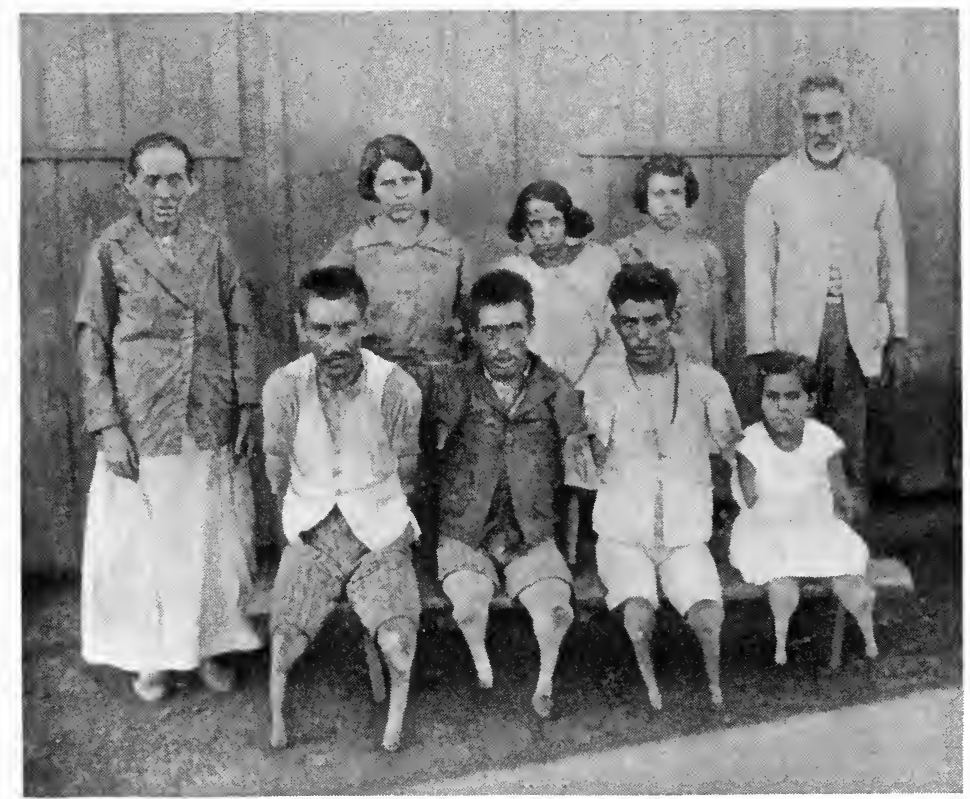

FIG. 39.-Family from Brazil showing hereditary absence of hands and feet. The man in the picture is the uncle of the children shown, but the father, who is no longer living, also had the same deformity. Of the twelve children in this family six were normal and six were deformed. (From Eugenical News.)

father and all three of the children there were neither hands nor feet. Other pedigrees have been described in which a hand or a foot was lacking through three generations.

An extraordinary number of hereditary defects have been observed in the eyes. Among these are atrophy of the optic nerve, misplaced lens, glaucoma, absence of the iris, cleft iris, nearsightedness, cataract, pigmentary retinitis, color blindness, night blindness, paralysis of the eye muscles, 
drooping eyelids, and various types of blindness. Some of these such as color blindness, night blindness, and atrophy of the optic nerve are usually transmitted as recessive sex-

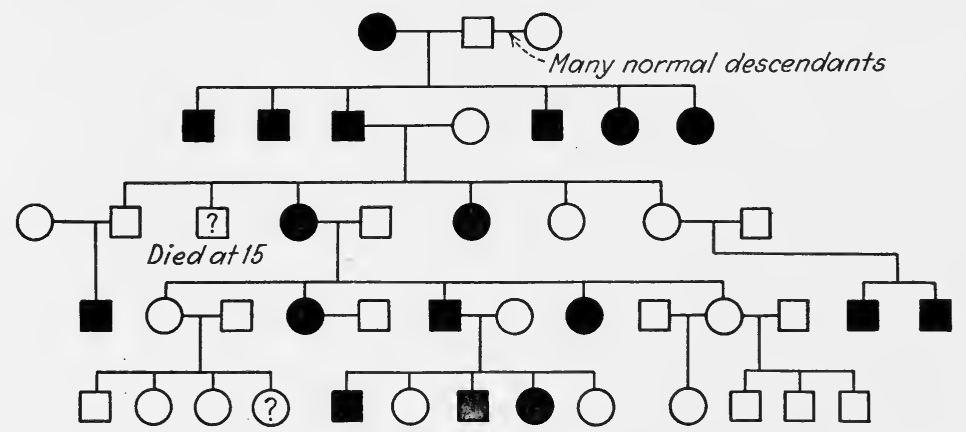

FIG. 40.-Dominant inheritance of glaucoma. The mother in the first generation developed glaucoma and became blind after some of her children were born. Her husband's descendants by a later marriage were all normal. All of the six children of the first generation suffered from glaucoma, but only one produced children, two of whom developed glaucoma. The trait was passed on through two of these children who were apparently normal and also through one who was glaucomatous. The trait commonly develops fairly late in life and some who did not develop it may have done so had they lived longer. In this pedigree glaucoma behaves like an imperfect dominant. (Data from Courtney and Hill.)

linked traits, but some pedigrees have been described in which color blindness and night blindness are sex-linked dominants.

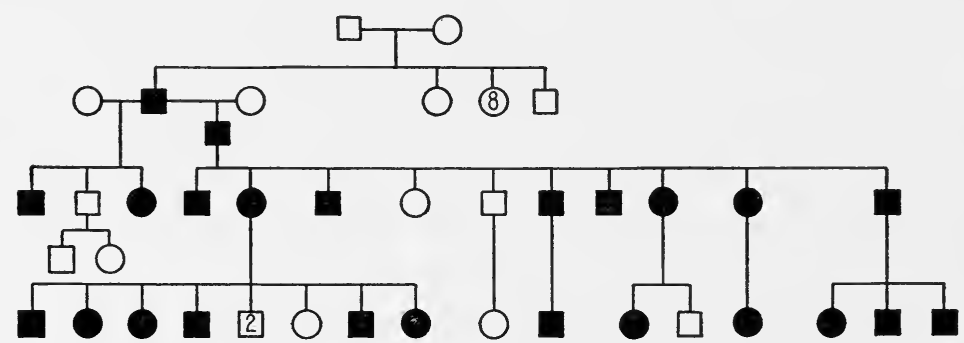

FIG. 4I.-The inheritance of cataract. The condition of the two original parents is unknown. The character here behaves as a dominant. Note that an affected male in the second generation has transmitted the defect to children by both wives. Where an individual is free from cataract the children are also free. In some pedigrees children inherit cataract from parents who do not manifest it in some cases because they died before the defect had developed. (After Nettleship.)

Deafness, although not infrequently caused by infections, such as scarlet fever, meningitis, measles, and syphilis, is in other cases transmitted as a recessive trait. Dr. Alex- 
ander Graham Bell in his memoir on The Formation of a Deaf Variety of the Human Race calls attention to the tendency of the deaf to marry the deaf, and the danger that deaf children may result from such unions. Even when both parents are deaf, all the children may be normal, but in other cases they are all deaf. In the former case, the deafness of one or both parents may have been secondarily acquired, and in the latter both parents may owe their defect to the same recessive gene. Where recessive deafness is present in a stock it is apt to be brought out by consanguineous marriages. In a number of inbred communities deafness is

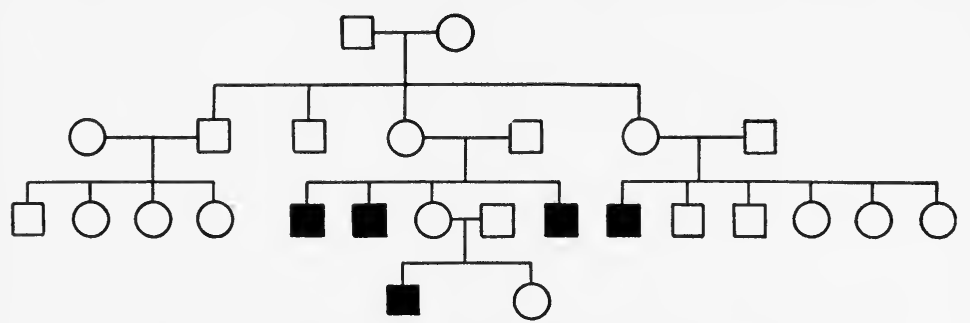

FIG. 42.-Inheritance of red-green color blindness. Only the males are affected but the trait is passed through females. (After Groenouw.)

common. According to Bell one out of twenty-five of the population of the western part of Martha's Vineyard in I 880 was a deaf mute.

There are several forms of hereditary deafness. In most of these the individual is born deaf, but congenital deafness is not always due to heredity. In many families deafness comes on slowly, leading to almost complete lack of hearing late in life. Where this is due to otosclerosis the condition of ten appears to behave like a dominant trait. There are different hereditary types of deafness which may be inherited in different ways.

As is abundantly illustrated in Cockayne's recent volume on Inherited Abnormalities of the Skin our integument manifests a considerable variety of hereditary disorders, 
quite aside from the anomalies of hair and pigmentation. The curious condition called ichthyosis (fish skin) in which the skin develops hard scales or bony plates is often transmitted as a somewhat variable dominant. In some pedigrees it seems to show sex linkage, appearing in males and not in females. Keratosis, a thickening of the palms and soles, is transmitted as a sex-linked recessive. The "porcupine man" whose horny skin was shed every year is said to have had six children who shared his peculiarity. The trait was continued for two additional generations.

Diabetes is commonly transmitted as a recessive trait. Since diabetes is more apt to occur in people who consume a large amount of carbohydrates the problem of its hereditary transmission is more or less complicated by the influence of the environment. Many hereditary traits manifest themselves as proclivities, or diatheses to disease without necessarily producing that disease except under certain conditions. In both plants and animals disease-resistant and disease-susceptible strains are well known. Susceptibility exists in all degrees and whether a disease is contracted may depend upon the dosage of the germs or the vitality of the individual exposed to infection. Frequently the immunity of a strain may be broken down by repeated or very heavy inoculations of pathogenic bacteria. Wright and Lewis have demonstrated by controlled breeding experiments that some strains of guinea pigs are much more susceptible to tuberculosis than others, and this has been found true for different breeds of cattle. A breed of sheep in Algeria is resistant to anthrax, which is highly fatal to ordinary varieties. Domestic fowl are ordinarily immune to anthrax, probably because their bodily temperature, which is higher than that of mammals, is too high to permit the propagation of the anthrax bacilli. In a famous and spectacular experiment Louis Pasteur demonstrated that 
chilling fowl by immersing them in cold water caused them to contract anthrax when they were inoculated with this disease. The germs multiplied abundantly in the blood of the infected birds and caused the death of those left exposed to cold. The birds put in warm surroundings, which quickly raised their temperature, recovered from the disease.

Hereditary diatheses are shown not only in relation to diseases caused by germs, but to disease provoked by other exciting causes. It has been shown that heredity plays an important role in asthma and hay fever, which may be occasioned by pollen, food, and a great variety of other substances to which the individual may have become sensitized. The studies of Adkinson on a large number of pedigrees point to the conclusion that the diathesis to asthma is a recessive trait.

The role of heredity in causing cancer has long been a matter of speculation. Statistical investigations carried on under the Imperial Cancer Research Fund showed that there are apparently no more cancers in the parentage of persons dying of this disease than in the rank and file of humanity. Accurate pedigrees showing the incidence of cancer in successive generations are difficult to obtain because a generation or two ago diagnosis was so imperfect that probably most deaths due to cancer were not so recorded. If a trait is caused by recessive genes that are sparsely scattered through the population, most individuals showing the trait will come from parents who do not show it, just as most black sheep in a flock come from white sheep. A parent-offspring correlation in such a case would be exceedingly weak. Since cancer is a disease of old age many individuals who might be due to die of cancer because of their heredity are carried off in the meantime by some other malady. The investigation of the hereditary factor in cancer by the collection of mass statistics is therefore 
very unsatisfactory. Many pedigrees have been collected showing that cancer appears with unusual frequency in certain families, but with all humanity to draw from one might collect many such cases purely as a result of chance association. There are some cases, however, for which this explanation seems improbable. Dr. Warthin has described one family in which twenty-seven cases of cancer, mostly of the stomach and intestines, appeared in three generations. Purtscher has described the case of a family in which several

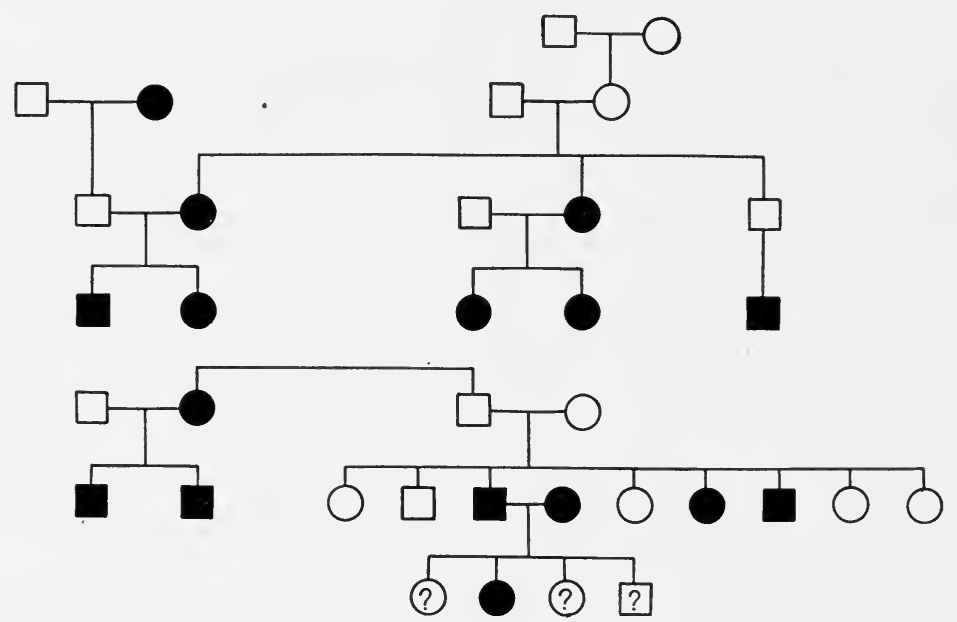

FIG. 43.-Two pedigrees of cancer. ?, persons too young to manifest cancer. (After Rice.) members died of a rare cancer-like growth known as retinal glioma. The father who died of sarcoma had eleven children, three of whom died of retinal glioma. One of these, a daughter, married and had a boy who died of the same malady, and there were two other cases among the children of members of the family who remained free of the tumor, but who were apparently carriers of the disease. The one thing that is clearly established about the causation of cancer is that cancers are apt to be produced by chronic irritations. A suggestive case is afforded by three brothers, all smokers, who developed cancer of the lip in middle life. A fourth 
brother did not smoke, but he nevertheless developed cancer of the lip in his sixty-fifth year.

In animals diatheses to cancer are clearly demonstrable. Miss Maude Slye, who has spent many years in studying cancer in mice, has developed strains of mice showing various degrees of cancer mortality from $\circ$ to Ioo per cent. According to Miss Slye cancer in mice is a recessive Mendelian trait, but whether one or more genes are involved is a matter of dispute. In different strains cancer shows a marked proclivity to attack particular organs such as the lungs,

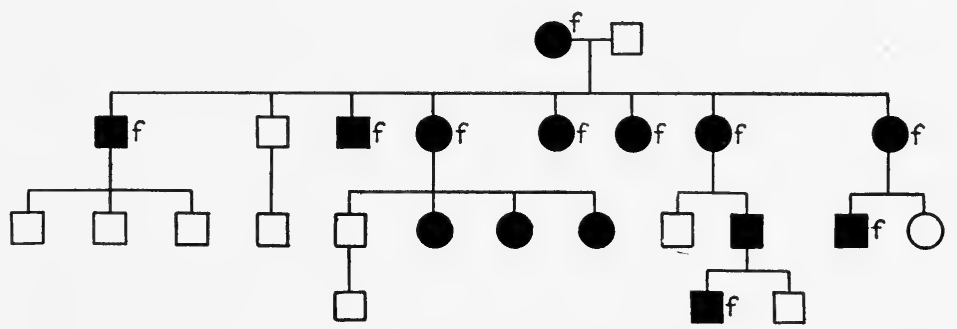

F1G. 44.-Family with blue sclerotics. Individuals marked $f$ also with fragile bones For some peculiar reason these unlike characters are closely associated in heredity. (After Burrows.)

liver, skin, and mammary glands, and the same tendency is indicated by many pedigrees of human beings.

It would require a large book to give an adequate account of hereditary human ills. Defective genes produce the greatest imaginable variety of effects. They may manifest their influence in a misplaced lens, a dislocated hip, atrophy of the optic nerve, short stubby fingers, a proclivity to bronchial asthma, deafness, blindness, muscular atrophy, fragility of bones, hemophilia, harelip, baldness, excessive hair, supernumerary teeth, or lack of teeth, dark-colored urine, or a small harmless pit in the lobe of the ear. These and numerous other departures from normality are all the products of a long chain of effects and a complex series of interactions of parts. Truly, we are fearfully and wonderfully made. 
One of the noteworthy peculiarities of human heredity is the variability with which a given trait is manifested in different pedigrees. Now a trait is clearly dominant. In other cases it appears to be recessive, or again it may be partially dominant, occasionally skipping a generation. Hereditary characters may vary greatly in their degree of development, as we have seen in the case of polydactylism. They also vary in their qualitative expression. In seeking an explanation for this feature of human heredity we should bear in mind two facts: (I) Characters which are much alike may be due to different genetic factors and hence may show different modes of inheritance. In the fruit fly Drosophila genes affecting eye color are scattered about in different loci of the chromosomes. So also are factors affecting wing venation, body color, bristle development, and size of body. Hereditary blindness in human beings is brought about by several kinds of defects. It is not improbable that different cases of hereditary deafness, color blindness, and various other hereditary defects in man are due to quite different genes.

(2) Even where defects are known to be due to the same gene, there may be several mutant changes in one gene as in the locus for white eye in Drosophila, which produce more or less similar manifestations; but the greatest cause of hereditary diversity is due to the varied company of other genes. Every character is the product of many factors. Let us suppose that a given gene is responsible for the development of insanity sometime after middle age. If the gene in question has to work out its effect in company with other genes causing migraine, spastic paralysis, or mental deficiency, the manifestations of insanity. would doubtless vary to a considerable degree. One might compare the effects of a gene in different constitutions with the growth of a plant in different kinds of soil and in company with different kinds of plants. We cannot expect all so-called unit characters to be invari- 


\section{IIO HUMAN GENE'TICS AND ITS SOCIAL IMPORT}

able any more than we can expect all plants of a given variety to be the same in all kinds of environments and associations. A plant grown amid others may not only fail to attain its normal size, but it may undergo changes of form and habit of growth. Gene effects are never produced singly. The action of every gene is conditioned by the actions of multitudinous other genes. And since the genes of the human species constitute an exceedingly varied company, it is inevitable that each gene should express itself in many different ways. This is especially true of those genes which affect complex traits such as the peculiarities of human behavior. It is scarcely conceivable that insanity or epilepsy should present the same degree of uniformity as might be found in eye color or in the tints of the skin, and if these complex traits are highly variable as a result of diversities of genic composition, they may be expected to manifest a great deal of variation also as a result of environmental influences.

The relation of environment and heredity will be treated in the following chapter.

\section{Suggested Readings}

Lindsey ('32), chap. 17. Pearson ('27). Shull, ('31), chaps. 21, 22. For further information on special topics see Baur, Fischer, Lenz (' 31 ), Blacker ('31), Crew ('27), Davenport ('i I), Gates ('29).

\section{Questions}

I. In studying human pedigrees, how can you tell whether a given character is dominant or recessive?

2. If a congenital deaf mute marries a person who became deaf through inherited otosclerosis, what would probably be the condition of the children?

3. Make a list of twenty human traits indicating the mode of inheritance in each case.

4. Do you think it likely that a trait may be inherited in a monohybrid ratio in one strain and in a dihybrid ratio in another?

5. Why do you think that a trait due to a single pair of genes should present many variations in its manifestation in different lines? 
6. Would a dominant sex-linked character appear more frequently in males or in females?

7. How do Negroes and whites differ in their resistance to tuberculosis, pneumonia, scarlet fever, erysipelas, and most diseases of the skin?

8. What can you say as to the probable number of genes in which whites, Negroes, and Mongolians differ?

9. In what respects do you think that you differ hereditarily from other members of your family?

I0. A student registering for a course in genetics was asked, "How many times have your bones been broken?" The reply was, "Three." What led the instructor to suspect that the student had fragile bones?

II. If a normal-haired woman with a bald father marries a normalhaired man, what may be expected of the children?

12. If a very rare trait appears in three generations of a family, what would you conclude as to its probable mode of inheritance?

13. If a trait is dominant, sex-linked, and lethal when homozygous or when not counteracted by a corresponding normal factor, what would be the sex ratio of the offspring of mothers showing this trait? 


\section{CHAPTER X \\ HEREDITY AND ENVIRONMENT}

WHETHER heredity or environment is chiefly responsible for the development of this or that trait has been the subject of no end of controversy. We have the hereditarians who are accused of attaching altogether too much importance to the germ plasm, and the environmentalists who like to think that heredity counts for little especially in the affairs of men. On this question there are all grades of opinion between the extremes of both camps.

It is quite natural that the doctrine that all men are created free and equal should make a strong appeal to the champions of equal rights. The teachings of Rousseau, the founders of our own government, and other leaders who strove valiantly in the cause of human freedom during the latter part of the eighteenth century, emphasize the influence of environment and opportunity in bringing about the observed inequalities among human beings. Mr. John Stuart Mill, whose humanitarian spirit pervades all his writings, remarked that "of all vulgar modes of escaping from the consideration of the effects of social and moral influences on the human mind, the most vulgar is that of attributing the diversities of conduct and character to inherent natural differences"- a statement which is supported with cordial approval by Mr. T. H. Buckle in his History of Civilization. In a similar vein Henry George declared that "the influence of heredity, which it is now the fashion to rate so highly, is as nothing compared with the influences which mold the man after he comes into the world." As representing the opposed standpoint of the hereditarians we may quote 
the matured opinion of Francis Galton that "nature prevails enormously over nurture when the differences in nurture do not exceed what is commonly found among persons of the same rank of society and in the same country."

Heredity and environment represent two large groups of factors, which are concerned in the production of all characters. In one sense neither is more important than the other because both are all-important. We can only compare the effects of differences in heredity with the effects of differences in environment. When we properly limit our inquiry it is often possible to arrive at a definite solution of our problem. In many cases the preponderating influence of hereditary or environmental differences is clear. Whether a man has a black or a white skin, blue eyes or brown, straight or kinky hair, depends mainly upon his allotment of genes. Whether he speaks English, Russian, or Chinese depends mainly upon his social environment. But even the color of a man's skin or eyes, and the degree of kinkiness or straightness of his hair may be affected to a slight extent by environmental forces, and whether he speaks a given language well or poorly, or is even able to learn a language at all, may depend upon his peculiar heredity.

It is evident that each character presents its own peculiar problems with reference to the relative influence of nature and nurture. Many problems of this kind are difficult to solve, because of the imperfection of our measures of the hereditary or environmental influences involved. We know in a general way that both hereditary and environmental differences affect stature, weight, general health, longevity, intellectual aptitudes, and traits of character, but in a mixed population of human beings whom it is not feasible to subject to experimental tests, it is difficult to arrive at an accurate measure of the extent to which the variability of these traits is to be attributed to genetic or environmental factors. 


\section{4 HUMAN GENETICS AND ITS SOCIAL IMPORT}

As we learn more of the mechanism of development, we find that the environment influences organisms in unexpected ways. Without a sufficient supply of iodine an otherwise comely and intelligent child might be transformed into a coarse, stunted, and repulsive-looking imbecile. Not improbably a similar result might be produced by a change in a gene which would check the development of the thyroid gland. As a matter of fact thyroid deficiency is to a certain extent a hereditary character. One of the most striking
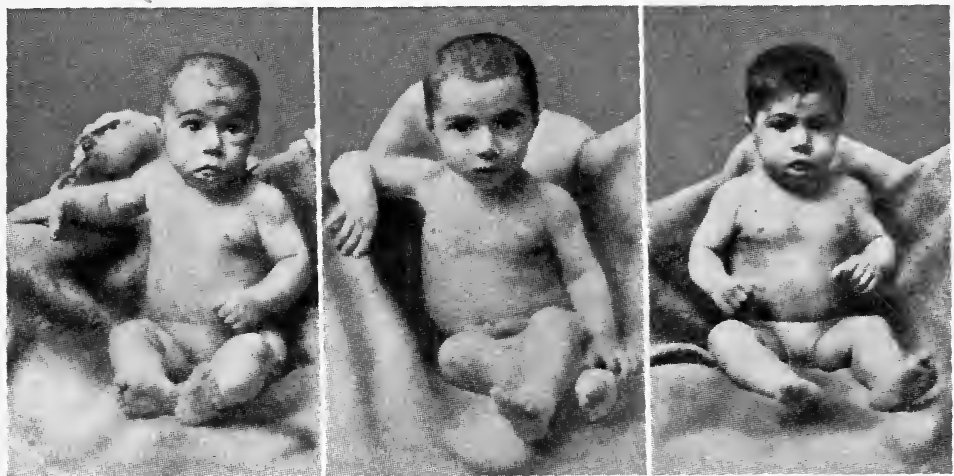

FIG. 45.- Three photographs of the same child suffering from thyroid deficiency. Before treatment with thyroid extract growth was stunted and abnormal. After three weeks of treatment the child made remarkable improvement as is indicated by the middle figure. The parents then had the thyroid treatment discontinued and before long the symptoms of cretinism began to reappear. In six months the child presented the appearance shown in the right hand figure. (Copyright by fournal of Heredity.)

achievements in the study of the glands of internal secretion is the discovery that children suffering from thyroid deficiency may be converted into normal, intelligent human beings by the administration of thyroid extract. Both heredity and environment are capable of producing much the same kind of developmental defects. For this reason, the shortcomings of heredity may in some cases be compensated for by supplying the proper environmental influence.

If we knew just how genes exert their influence upon the developing organism, we might be able to overcome many hereditary defects. Some kinds of dwarfism are probably 
due to a defective development of the anterior lobe of the pituitary gland. In fact, some cases of dwarfism have made a satisfactory growth under gland treatment, but whether the dwarfism was an inherited defect is not known. Even sex, which is commonly determined by the sex chromosomes, may sometimes be changed by environmental factors. What under ordinary circumstances is determined by heredity, under extraordinary circumstances may be determined by the environment. Both heredity and environment are of course essential for the production of either a male or a female. In this case environment determines whether the male-producing or the female-producing genes, which are present in every individual, assume the predominant role. The so-called environmental determination of sex is quite analogous to the environmental determination of dominance in a heterozygous individual.

Possibly the future may give us the means of controlling the activities of genes to a much greater extent than seems likely today. We may find substitutes for gene deficiencies, so that people can get along without several kinds of genes which are now essential. But the more we are able to compensate for gene deficiency, the more we become dependent upon the environment for the maintenance of life and health. The cretin must continue to take his thyroid tablets or lapse back into imbecility. We might imagine a remote future in which people would have to be continually dosing themselves with gene substitutes in order to keep alivewhich on occasion might prove to be a very precarious situation, to say nothing of its other drawbacks.

What we are interested in knowing are the kinds and degrees of effects which may be produced by a given environmental change, or a given change in hereditary constitution. Under certain conditions, such effects may be measured with a fair degree of accuracy. If I have a uniform lot of 


\section{II6 HUMAN GENETICS AND ITS SOCIAL IMPORT}

beans derived from a pure line, I can measure the effect of applying a certain kind of fertilizer, provided I keep all other conditions the same which might influence the growth of the plants. I might also measure quite accurately the effect of genetic differences between two varieties by raising all the plants under identical conditions of nurture. Then I could compare the genetically caused differences with those due to environment and determine which were the greater. But whether the one or the other produced the greater effect would depend upon the particular genetic and environmental influences I chose to compare. I might sow some seed in very rich soil, and some on stony ground and obtain enormous differences in yield due to environment. Or again, I might choose strains genetically much alike, or again very different, and thus obtain a wide range of results due to hereditary diversity.

The question of the relative influence of hereditary and environmental differences has been investigated for a number of human traits by Pearson, Elderton, and other biometricians, and they have come to the conclusion that for the traits studied heredity is much more potent than environment in causing the observed differences. The method employed is to work out correlations between related individuals for the various traits. These are assumed to give a measure of the potency of heredity. Correlations are also worked out between these traits and various environmental factors. If it is found that a parent-offspring correlation is of the order of 0.5 , as it is for many characteristics, and the environmental correlations are much weaker, such as 0.1 or 0.2 , the conclusion is drawn that heredity is much more potent than environment in causing the observed differences.

As we have already seen, a parent-offspring correlation is not necessarily, or even usually, an adequate measure 
of the strength of heredity. Were we to study the relative effects of hereditary diatheses and environmental exposure upon the occurrence of tuberculosis, we could not assume that a parent-offspring correlation of 0.5 which was found by Pearson and Goring is necessarily due to heredity. It might, as has been claimed, be due to the transfer of infection or exposure to a common environment.

It should also be pointed out that not only may parentoffspring correlations be due to environment instead of heredity, but correlations with environmental differences may be due to heredity instead of environment. If I were to study the effect of an urban environment upon stature and measured a considerable number of Italians (who are a relatively short people) in New York City and an equivalent number of the old American stock (who are fairly tall) in the rural areas of New York State, I might be betrayed into concluding that a difference due mainly to racial heredity was caused by conditions of life in a large city. Theoretically, coefficients of correlation may throw much light on the nature-nurture problem, but they have to be employed with caution, and with an ever-watchful eye to alternative possibilities of interpretation.

A valuable side light upon our problem is furnished by the study of twins. Francis Galton was the first to recognize that twins fall into two classes: (I) ordinary, or fraternal twins, who may or may not be of the same sex, and are not much more alike than other brothers and sisters; and (2) identical twins, who are usually very much alike and are always of the same sex. It is now fairly well established that identical twins come from the same fertilized egg. They are analogous to the quadruplets that arise from a single egg in the armadillo after it has undergone a certain degree of development as a single individual. Unless some unusual departure from ordinary chromosome behavior occurs 


\section{8 HUMAN GENETICS AND ITS SOCIAL IMPORT}

during early development, identical twins would have precisely the same heredity. Often their similarity is so close, that their friends and sometimes even their parents have difficulty in telling them apart. They are living testimonials as to the potency of heredity as a cause of likeness.

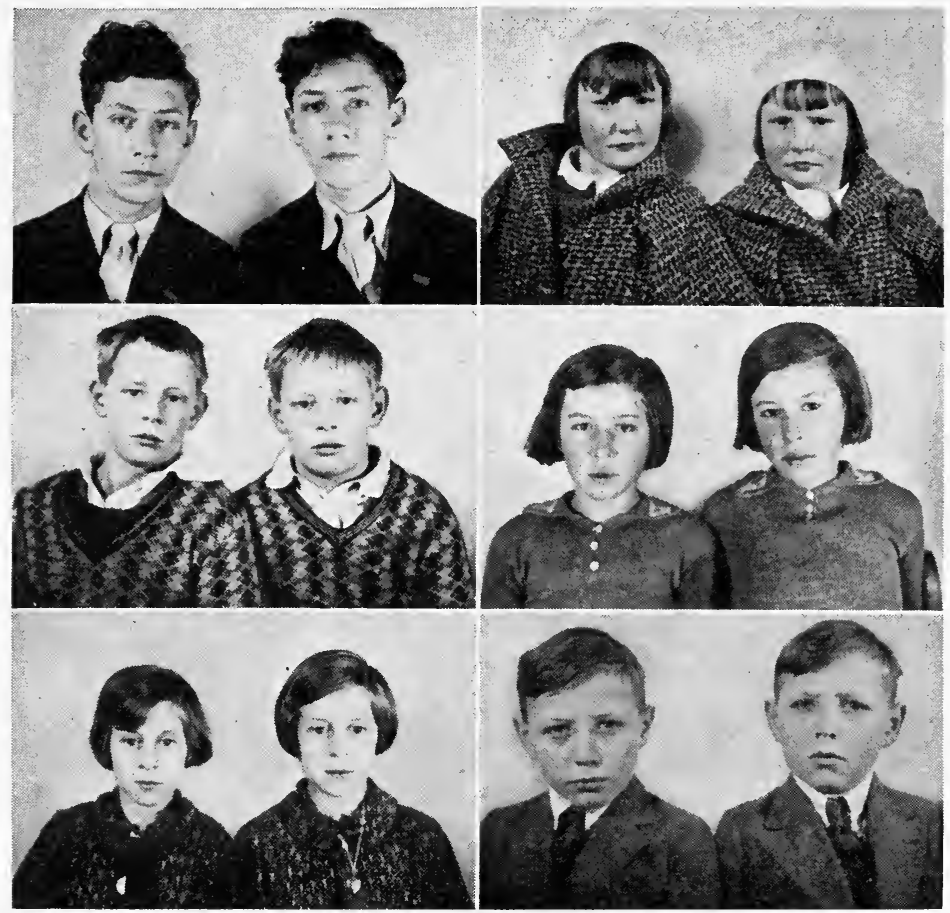

Fig. 46.-Six pairs of identical twins. In all these pairs both members belonged to the same blood group and had almost exactly the same shade of eye color and hair color. Both of the girls of the lower left pair had only two upper incisor teeth. In both boys in the lower right figure the second and third toes of both feet are joined by a fleshy web. (From photographs by $\mathrm{Dr}$. R. Stohler.)

It is commonly assumed that environmental factors are on the average the same for identical and fraternal twins, and hence we have the opportunity of comparing like and unlike heredity under the same environmental conditions. On the other hand, it may be claimed that identical twins are, ipso facto, more apt to be exposed to similar influences, 
and hence tend to become more nearly alike than fraternal twins. The only actual investigation of this question has been carried on by Dr. Paul Wilson in a fairly large number of twin pairs, and he finds that identical twins as compared with fraternal twins of the same sex, more often dress alike. are more frequently in the same grade and room in school, have more friends in common, are less frequently separated, and have more similar tastes in food and more similar records for diseases and for general health. Differences in environmental exposure are greater between twins of unlike sex than between identical and fraternal twins of the same sex. This is a very important fact since differences between identicals and fraternals of the same sex are commonly much greater than differences between fraternals of the same sex and fraternals of different sex.

If we could experiment freely with human beings it would be very interesting to compare the results of the following combinations:

Like heredity under the same environment;

Like heredity under different environments;

Unlike heredity under the same environment;

Unlike heredity under different environments.

Since we cannot deal with human beings as we do with guinea pigs, we have to select those cases which approximate most closely to the conditions specified. There are many instances of identical twins exposed to environments which are very similar. Of course, the environment is never quite the same for two human beings even when they are reared in the same home and attend the same classes at school. One twin might contract a disease, experience a nervous shock, or receive an inspiration through personal influence which might have a marked effect upon the development of physique or character. These inevitable environmental 
differences affect some characteristics much more than others. In eye color, hair color, configuration of the external ear, head index, general body build, texture of skin, dentition, vision, hearing, and blood grouping, the similarities of identical twins are as a rule very striking and very much closer than those of fraternal twins. Characters which are much affected by environmental factors show a greater degree of variation. There are several diseases which when they occur in one member of a pair of identical twins usually occur in both, whereas in fraternal twins they are commonly manifested in only one member of a pair. With other diseases, such as whooping cough and mumps, the distribution between identicals and fraternals is not greatly different.

The study of twins has proved very valuable in throwing light on the role of hereditary diatheses in various maladies. The influence of heredity in tuberculosis has long proved to be a peculiarly knotty problem, since the well known tendency of tuberculosis to run in families might be explained as a result of either heredity or environment. Diehl and Verschuer have recently compiled data on the incidence of tuberculosis in fraternal and identical twins and their results may be summarized in the following table:

\begin{tabular}{|c|c|c|c|}
\hline & Group A & Group B & Group C \\
\hline Identical twins. & I3 & 4 & .2 \\
\hline Fraternal twins of like sex..... & 8 & I 4 & I6 \\
\hline Fraternal twins of unlike sex... & 4 & 6 & 8 \\
\hline
\end{tabular}

In group $A$ are included cases in which both twins are tuberculous and in which the time of onset and course of the disease are similar. In group B both twins are tuberculous, but the disease differs in severity, time of onset, or organs affected. In group $\mathrm{C}$, one twin was tuberculous and the other not. Although the number of cases is not large the 
striking differences in the incidence of tuberculosis in identical and fraternal twins furnish strong evidence for a hereditary diathesis to this disease. One particularly impressive instance is furnished by a pair of identical twins in which tuberculosis developed in the bone of the heel of both members.

Notwithstanding many remarkable similarities, identical twins may present rather marked differences. Dahlberg has found that identical twins differ more in weight at birth than ordinary twins of the same sex, but in a short time the identicals show the greater similarity. This as well as other differences may be due to the fact that identical twins are more closely associated in utero than ordinary twins and tend to interfere more with each other's development. Accidental variations of position might result in unequal rates of growth, or in handicapping one member in some other way. If one twin is stillborn, the liability of the other member to be stillborn is about three times as great in identical twins as in fraternal twins. This may be due in part to their closer association and to the fact that there is a more intimate connection between the blood systems of identicals than between those of fraternals.

Another factor making for inequality is "mirror imaging" - the right side of one twin sometimes shows a tendency to resemble the left side of its cotwin. The degree to which identical twins tend to develop complementary asymmetries, may depend upon the stage at which they come to be separated. For a time both develop as one individual, and it seems not unreasonable to suppose, as suggested by Newman, that when separation is delayed mirror imaging becomes more pronounced. It is possible that this tendency might have far-reaching effects, not only upon physical traits but also upon personality. If, for instance, it caused one twin to be left-handed this circumstance might have a 


\section{I22 HUMAN GENETICS AND ITS SOCIAL IMPORT}

considerable influence on the development of the individual in several ways.

It is a curious fact that identical heredity should be associated with conditions which are provocative of differ-

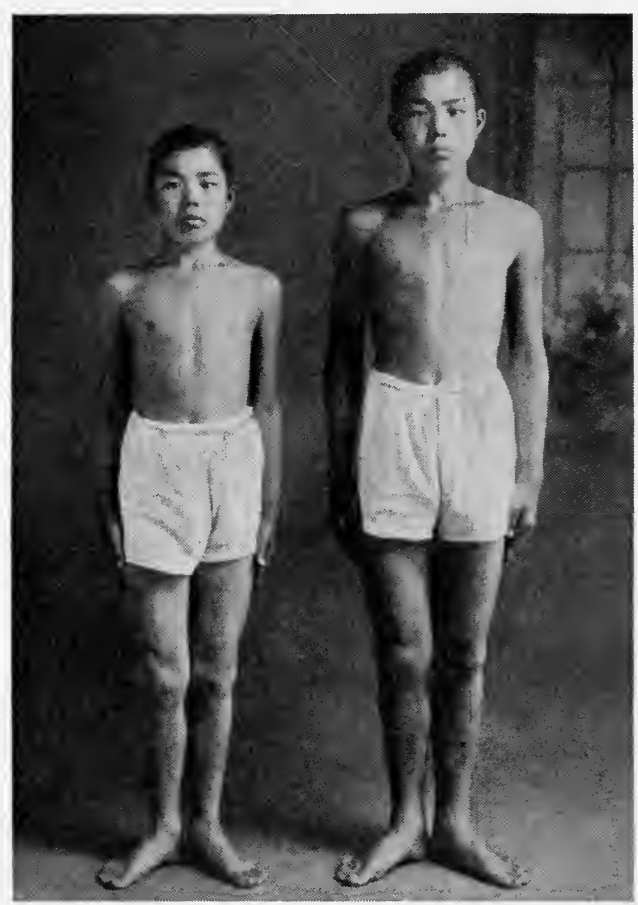

Fig. 47.- Japanese twins probably identical but showing unequal development. At birth they were attached to one placenta and had almost exactly the same weight and head circumference. Their finger prints and palm patterns are remarkably similar. Up to school age they could scarcely be distinguished. In their second school year they began to exhibit differences in growth. One twin $M$ developed diabetes insipidus and grew less rapidly than the other who was free from this malady. $X$-rays showed that the pituitary fossa was larger in the larger twin. The cause of the unequal development of the twins is unknown. (Copyright by fournal of Heredity.)

ences. How far identical heredity is directly responsible for the development of unlikeness in identical twins is uncertain. Mirror imaging is apparently more frequent in conjoined twins than in ordinary identical twins. Keeler found that among laterally joined double monsters, "ten out of thirteen asymmetrical pairs of characters, or 77 per 
cent are mirrored," while in fourteen pairs of identical twins only 22 per cent of such characters are mirrored. In conjoined twins complementary asymmetries are relatively frequent in internal organs, a condition which may

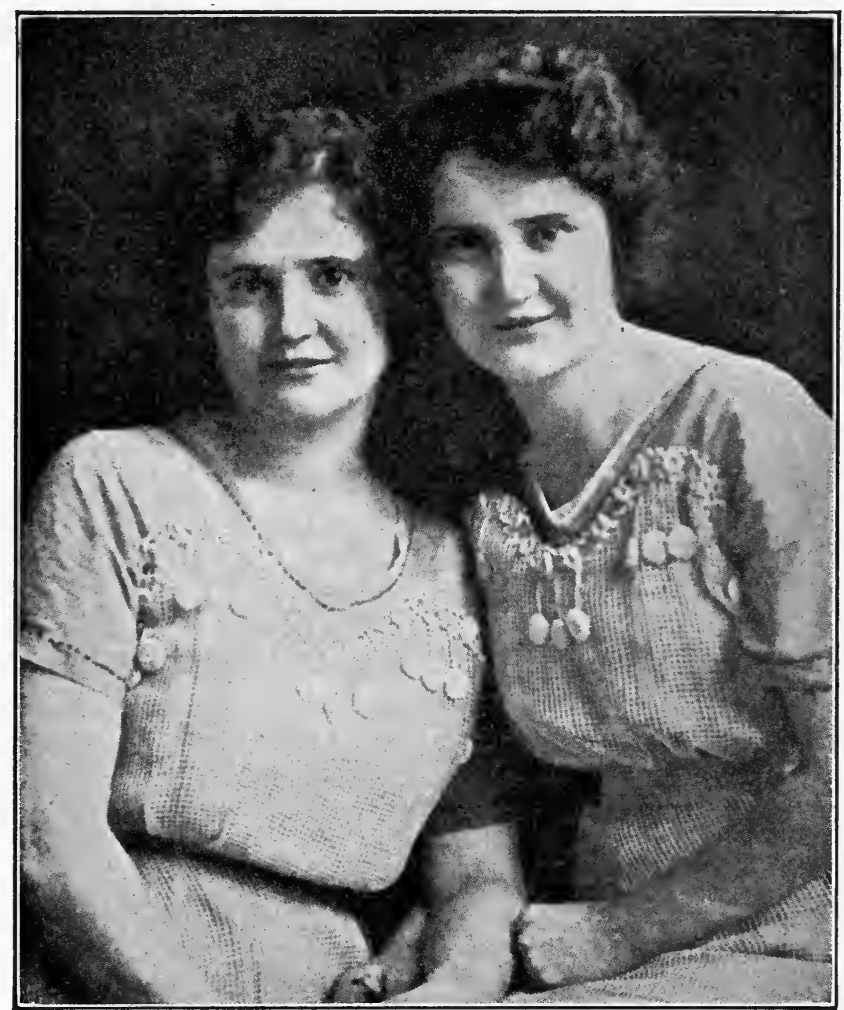

FIg. 48.-Identical twins reared apart. (From Popenoe in the fournal of Heredity.)

well be the occasion of physical maladjustments in one or the other individual.

Although so-called Siamese twins are often remarkably alike, there are some cases in which one twin is better developed than the other. Two Siamese twins, $D$ and $V$, studied by Koch differed in height by $4 \mathrm{~cm}$. "Consistent superiority is exhibited by $D$ [the taller one] in all the intelli- 


\section{HUMAN GENETICS AND ITS SOCIAL IMPORT}

gence tests as well as those of scholastic abilities, such as arithmetic, vocabulary, composition and reading comprehension," but the differences in the Army Alpha Tests were less than those of three-fourths of the average twin population and were about a third as great as the average variability of a fourteen-year-old population. In general, conjoined twins are less similar than identical twins who are separate. This may be due to a certain amount of interference during development together with a greater degree of mirror imaging.

\section{Suggested Readings}

Jennings ('30), chaps. 5, 6. Newman (' 32 ), chaps. 37, 38. Further references on twins, Dahlberg ('26), Hirsch ('30), Holzinger ('35), Lauterbach ('25), Muller ('25), Wingfield ('28).

\section{Questions}

I. Mention several characteristics of plants or animals which are greatly influenced by genetic factors and also by environmental conditions.

2. What can you say of the role of genetic and environmental factors in diabetes, asthma, and hay fever? $(-$

3. In what diseases of man do genetic differences play little part?

4. In what diseases of man do environmental differences play little part?

5. Do environmental factors ordinarily cause differences in height as great as those between Norwegians and African pygmies?

6. Which do you think is more affected by the environment, height or weight?

7. Would you class differences in hair color due to age as hereditarily or environmentally caused?

8. Is there any limit to the differences which environment might make between individuals of like heredity?

9. Is environment more effective in making like individuals unlike or in making unlike individuals alike?

10. Is it possible for two fraternal twins to have the same heredity?

II. In studying the relative roles of heredity and environment is it important to ascertain the differences environment can make or to con- 
sider the extent of the differences which arise under environmental conditions that are commonly met with?

12. What other reasons beside those mentioned might cause both members of a pair to die in utero more frequently in identical twins than in fraternal twins?

13. In what respect may freckles or sunburn be both a hereditary and an acquired character? 


\section{CHAP'TER XI}

\section{HEREDITY IN MENTAL DEFECT AND DISEASE}

\section{$A$ MONG the hereditary ills with which human beings}

$\mathcal{1}$ are afflicted those affecting the development of the mind are probably first in order of importance. These mental ills are of many varieties, but they may be grouped into two fundamental classes: (I) the lack of normal mental growth, or mental deficiency; and (2) abnormal mental development, or mental disorder.

Mental deficiency (amentia) may be due either to hereditary causes (primary amentia), or to environmental factors (secondary amentia). Occasionally a disease of childhood such as meningitis or encephalitis leaves a previously normal child with deficient intelligence. Congenital syphilis is sometimes responsible for mental defects as well as for many nervous disorders of children. Wassermann tests to ascertain the presence of syphilitic infection have been administered to the mentally defective inmates of several institutions, but the percentages of positive reactions reported were very unequal. If a mental defective gives a positive reaction for syphilis, it is no proof that the disease is the cause of the defect. Tredgold (Mental Deficiency) remarks that "on the whole, I doubt, whether syphilitic aments constitute more than 2 to 3 per cent of mental defectives." The percentage of syphilis seems to be greater in low types of mental defectives than in those of higher grade. In general, it is unlikely that syphilis is responsible for more than a small percentage of mental deficiency.

A small amount of mental defect is due to thyroid deficiency, inherited or acquired. Head injuries at birth and 
accidents during development are probably responsible for a larger number, although many cases of head injury and internal hemorrhage do not result in noticeable mental impairment. In a study of 20,473 cases of difficult labor Dayton found no relation between degree of difficulty and the degree of mental deficiency.

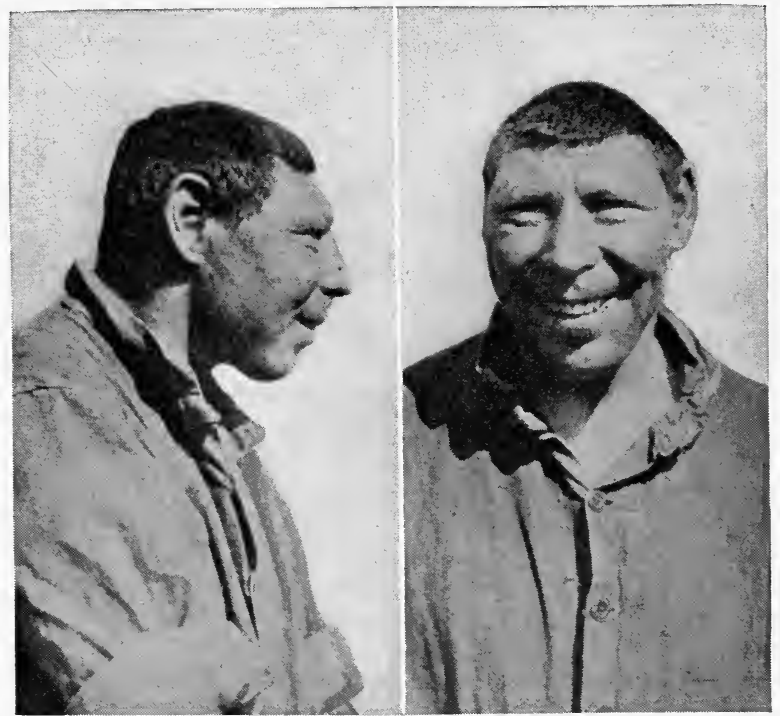

Fig. 49.-A microcephalic idiot. Although over thirty years old the mental age of this individual is about one and one half years. He can neither dress himself nor talk. (Courtesy Leon F. Whitney.)

Feeble-mindedness occurs in all degrees. Idiots, the lowest class, have an intelligence quotient from 0 to 25 , and their mental capacity does not develop beyond that of children of two to four years of age. The imbeciles have an intelligence ranging from 25 to 50 , and their intelligence corresponds approximately to that of children from three to seven years old. The morons who constitute the next higher grade have an intelligence quotient from 50 to 70 , or, as defined by the American Association for the Study of the Feeble-minded, from 50 to 74 . The morons grade into the 
borderline and dull-normal people, and the latter are connected by insensible gradations with individuals of normal and superior intelligence. Pearson and Jaederholm have contended that mental defect varies in a continuous manner. Both the idiots and the geniuses are represented by relatively few numbers. According to the findings of the Mental Deficiency Committee who made an extensive survey of certain regions of Great Britain, the idiots constituted only 3.6 per cent of the mentally deficient population; the imbeciles constituted I 6.6 per cent, and the feeble-minded 80.3 per cent. There are more borderline cases and dull

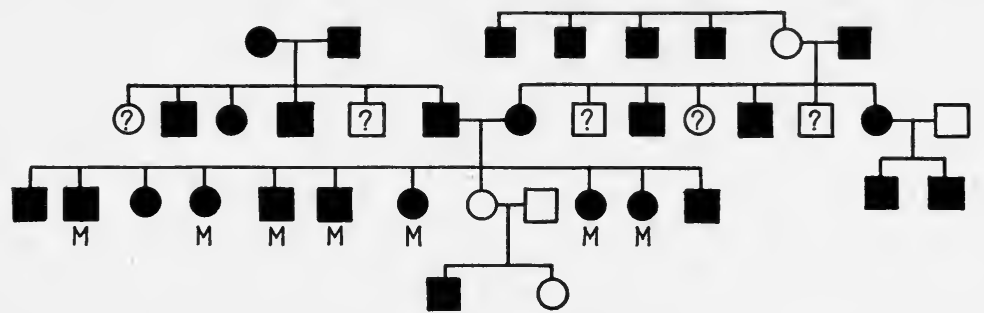

Fig. 50.-Pedigree of the $\mathrm{V}$ family showing mental defect and microcephaly. All individuals in black mentally defective; those marked $M$ are known to be microcephalic. The mental condition of those marked ? unknown. (After E. A. Whitney.)

normals than morons, while people of average ability constitute the most numerous class. The further they pass beyond mediocrity toward the highest types of intelligence the smaller the number of individuals. The distribution of intelligence resembles that of height or weight and approximates very closely a typical symmetrical frequency curve of variability (see Fig. 59). Only arbitrary distinctions can be made, therefore, between the various classes, whether of mental defectives or of geniuses.

The variability expressed in the typical bell-shaped curve is a product of many influences both genetic and environmental. The manifestations of mind are so varied in character and degree that it is evident that many genetic factors must be involved in their production. Feeble-mindedness 
has sometimes been considered as a simple recessive Mendelian trait, but there is much evidence that it is usually the product of a variety of genetic factors. There are probably some types of feeble-mindedness which depend primarily upon a single recessive gene. Among these is the peculiar condition known as amaurotic family idiocy. This defect is almost entirely confined to people of Jewish extraction. There is a degeneration of the retina leading to blindness.

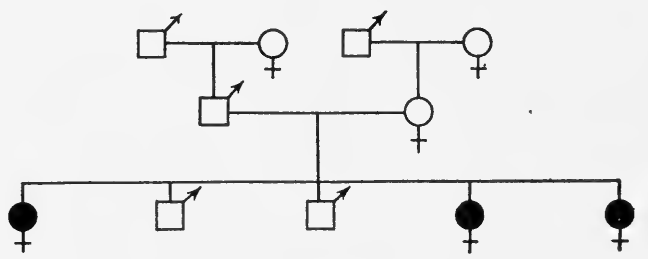

(a)

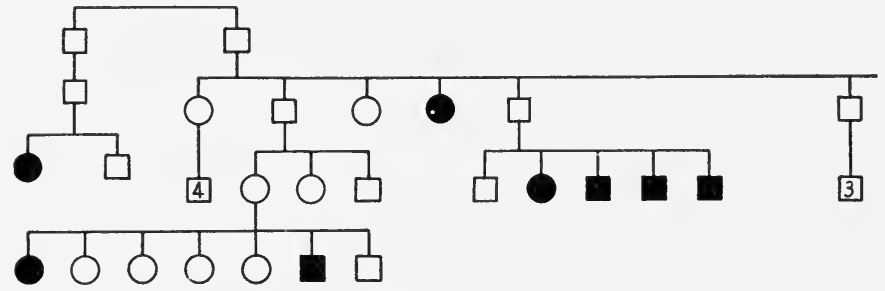

(b)

FIG. 51.-Pedigrees of amaurotic idiocy. In (b) all the affected individuals are descended from two brothers and both pedigrees show more than one affected person in a family. [(a) after V. Starck; (b) after Hermann.]

The condition involves grave nervous disorders and leads to death usually in the first year of life. Since the trait is lethal amaurotic children are always born to normal parents, but sometimes more than one case appears in a family. The defect is especially apt to be brought out in consanguineous marriages. For this reason, it is probable that it depends upon a recessive gene. There is a similar malady called juvenile amaurotic idiocy which appears in older children and also leads to imbecility and blindness. Although the 
defect is rare, it not infrequently affects more than one child in a family.

Another clearly recessive type of mental defect has been studied by Sjögren in an inbred community in Sweden. All the affected children belong to a clearly defined type of low-grade imbeciles. Most of them could not be taught to read or write; others learned very imperfectly, and they usually spoke indistinctly and only in monosyllables. One large group of interrelated families produced fortyeight of these imbeciles. Sometimes two or more appeared in the same family along with normal siblings. As a rule they were of good physical development, and the thirty-nine who were given Wassermann tests showed a negative reaction. On account of their low mentality these imbeciles were kept in homes or in institutions and did not propagate their kind. In every case the mental defectives were born to parents who were normal.

Writers on mental defects and diseases often make the assumption that if a trait is not found in the ancestors of an affected individual it is probably not due to heredity. There have been many estimates as to the percentage of cases of feeble-mindedness, epilepsy, and insanity which are due to heredity, the inference being that if the trait appeared in the ancestry in a certain percentage of cases, in that percentage, heredity could be assigned as its cause. Knowledge of the principles of Mendelian heredity shows the untenability of this view. Where a relatively rare recessive trait is involved, it may appear only very rarely in both parents and offspring. If the trait interfered with reproduction it might never appear in both parents and offspring. When we limit our standard of feeble-mindedness to cases of low grade we find that in most cases the feeble-minded come from ancestors classed as normal. Nevertheless, as in the group studied by Sjögren, the defects might be due 
HEREDITY IN MENTAL DEFECT AND DISEASE I3I

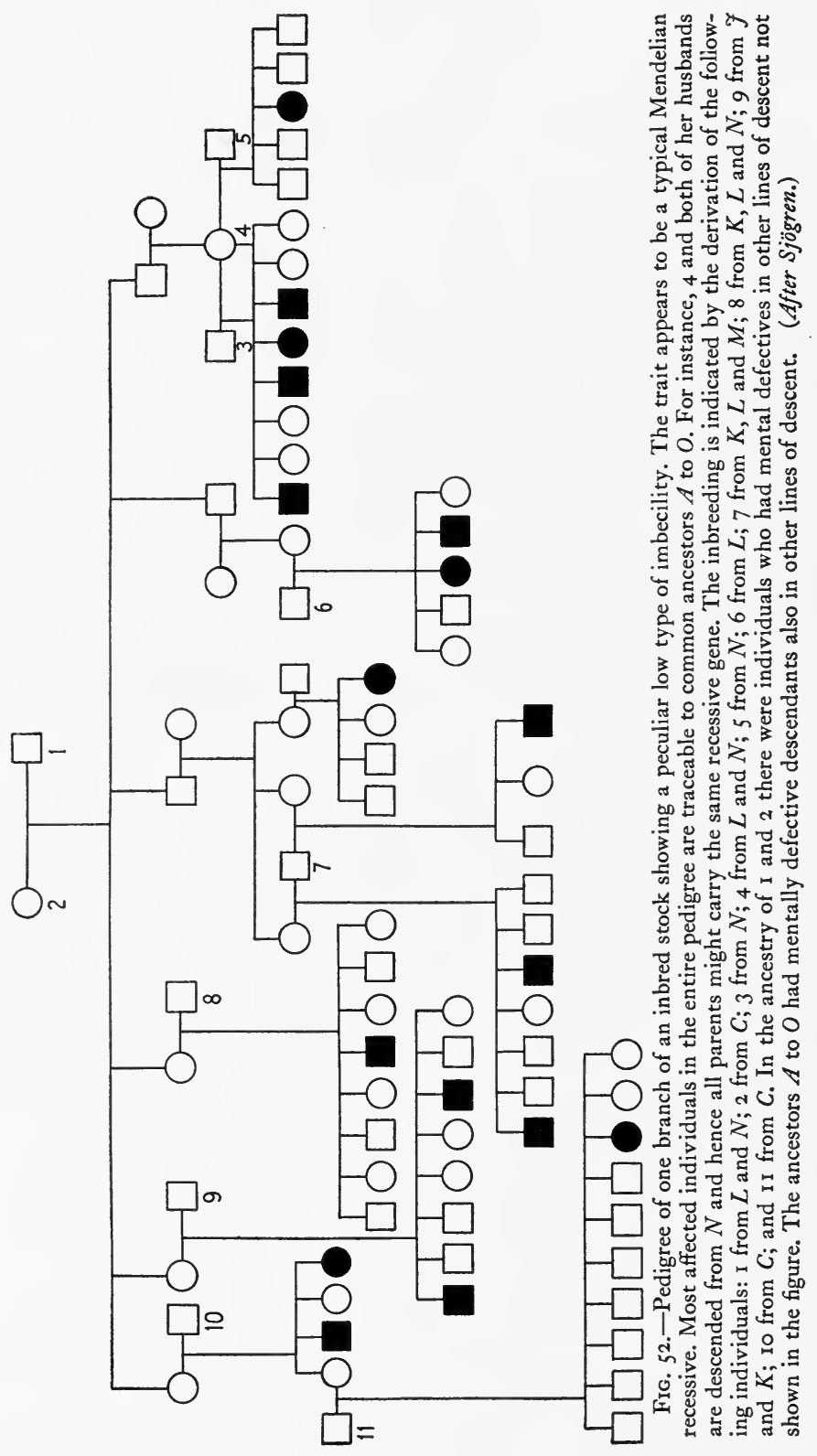




\section{I32 HUMAN GENETICS AND ITS SOCIAL IMPORT}

to genetic causes. 'The frequency with which a trait runs in families is not necessarily an index of the extent to which it is due to heredity.

It has been shown by the studies of the Mental Deficiency Committee and other investigations that there are fewer mental defectives among the parents of idiots and imbeciles than among the parents of the higher grades of the feebleminded. This is probably because there are a number of recessive genes each of which may give rise to mental deficiency of a low type. Such cases represent more or less pathological types of defect due to a pair of genes which profoundly disturb the course of normal development. The mental deficiencies of this type seem to stand apart from the normal gradations of mental ability much as a case of achondroplastic dwarfism stands apart from the normal fluctuations of human stature.

It is not the more or less clearly defined pathological cases of mental deficiency that constitute the greatest eugenic problem, because they are not a self-perpetuating class. The most serious problem is presented by the morons and the borderline cases who may be considered as representing the lower levels of the normal range of mental ability. Many individuals of this group pass as normal although rather stupid people. To a considerable degree they come from poor stock, although occasionally they may be born to very intelligent parents. According to several writers the feeble-mindedness of this class is inherited as a recessive Mendelian trait, but this conclusion has been criticized by a number of geneticists. In support of this theory is the fact that feeble-minded children are often born to normal parents, whereas if both parents are feebleminded practically all their children are feeble-minded also. According to Goddard, of the 220 children born of forty marriages in the Kallikak family in which both parents 
were feeble-minded all but two were feeble-minded also. In a larger series of 482 children of feeble-minded parents, all but six were said to be feeble-minded. Feeble-mindedness of the type usually found in degenerate human strains is no unitary characteristic. It is a highly variable character which can only be discriminated from normal intelligence by a purely arbitrary criterion. Most critical students of the genetics of mental deficiency are inclined to adopt the multiple-factor hypothesis, and it is not improbable that several of the factors involved may be dominant or partly dominant in their effects.

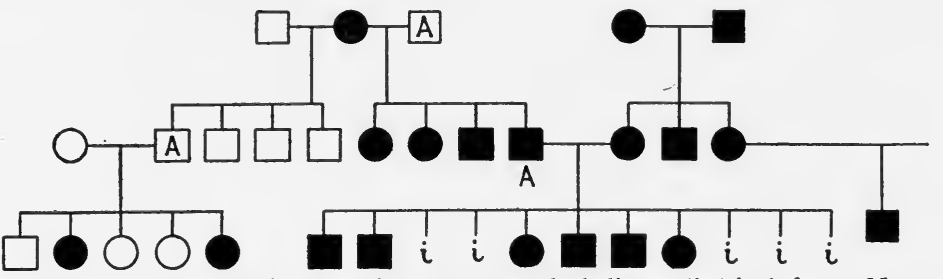

FIG. 53.-Inheritance of feeble-mindedness. $A$, alcoholic; $i$, died in infancy. Note that in the two cases in which both parents are feeble-minded all the children surviving infancy are feeble-minded also. (Data from Goddard.)

If intelligence is a multiple-factor character, we can understand why the matings of two people of low mentality usually produce only feeble-minded progeny, just as the mating of two small rabbits produces only rabbits of small size. Unquestionably children are frequently more intelligent than either of their parents. They may derive from each of their parents the genes most conducive to mental development, and hence rise higher than their source. But if children have a rather poor lot of parental genes to draw from, it is not likely that they will obtain a chance combination which will enable them to achieve a very high degree of intellectual distinction. The usual charts illustrating pedigrees of mental defectives give a rather misleading picture of the real situation. In the charts people are either normal or feebleminded, whereas they present a series of intergrading 


\section{I34 HUMAN GENETICS AND ITS SOCIAL IMPORT}

conditions which might be classed differently by another observer. Crosses between rabbits of different sizes give rise to a varied progeny according to the different combinations of genes influencing growth. Large rabbits usually have large offspring and small rabbits have small offspring. Matings of intermediate sizes give rise to rabbits of different size. If we had to designate every rabbit as either large or small as we designate people as normal or feeble-minded, we would get pedigree charts much like those illustrating the inheritance of mental defects.

The multiple-factor hypothesis enables one to account for the frequency with which feeble-minded children result from matings in which one parent is feeble-minded and the other normal. Not improbably many of these so-called normals who marry into feeble-minded stock are not very much superior to their mates. It is natural that their children with their varied allotments of genes should in some cases be above the standard for normality and in other cases below. If we adopt the hypothesis that feeble-mindedness is a recessive character, we should have to assume that a large proportion of the general population is heterozygous for mental defect.

A very instructive investigation of the origin of mental defectives has been made by Dr. Penrose on one hundred mental defectives not of the pathological type. The mental capacities of the parents, so far as they could be ascertained by careful study, were graded into the classes of superior (including persons of normal intelligence), dull, and defective. A similar rating was made of the capacities of the brothers and sisters of the offspring studied. The results are expressed in Table $\mathrm{I}$.

As is shown by the table the proportion of defectives increases as the intelligence of the parents decreases. The lowest ratio of mentally deficient children is found where 
both parents are of superior or normal intelligence. Where both parents are defective most of the children are defective or dull, only one being considered of normal mentality. While children may rise higher than their source they are much less apt to rise very high if their source is low. The familial distribution of intelligence shown in the table is about what might be expected if differences in mental development are largely determined by the varied assortment of genes. Some of these cases may, of course, be due to environmental causes, although environment does not readily account for the fact that defective parents are particularly prone to produce defective children.

Table I.-The Parentage of Mental Defectives

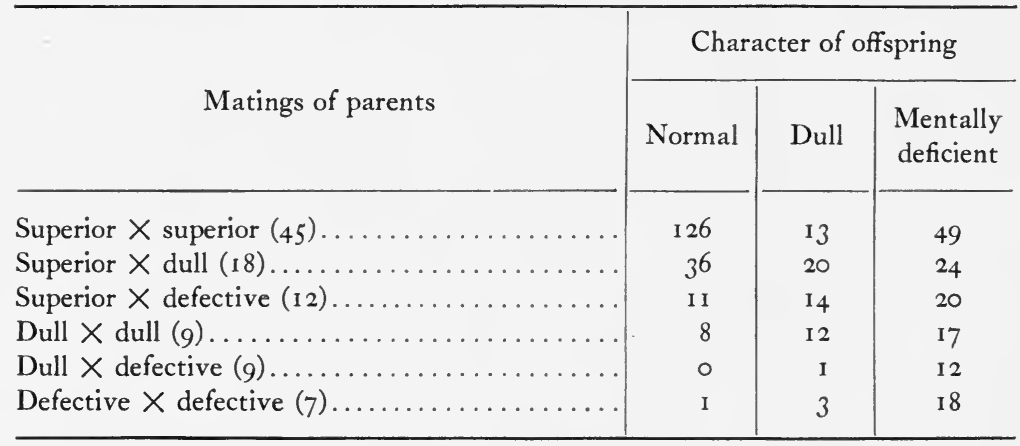

Some very pertinent data on the role of heredity in causing mental defects are furnished by recent studies on twins. Luxenburger has summarized the data on mental deficiency in identical twins up to I930, and he finds that out of eleven cases in which mental defect occurs both twins were defective in ten cases and showed a similar type of defect. In four cases in which feeble-mindedness occurred in indubitably fraternal twins, in all cases it affected only one member of the pair. In six instances in which the identity of the twins was doubtful both twins were affected in four cases. Dr. J. C. Smith found sixty-six pairs of twins 


\section{I36 HUMAN GENETICS AND ITS SOCIAL IMPORT}

among the 6,700 registered mental defectives in Denmark. In fourteen out of the sixteen cases of identical twins both members were feeble-minded, and there were two in which the mental defect was limited to one member. In the fraternal twins of the same sex the mental deficiency was clearly similar in kind in only one of the fifteen pairs. The proportions were much the same, three similar and thirty-two dissimilar, in the fraternal twins of opposite sex.

Results equally striking have been reported by Humm who found that in thirty-two pairs of identical twins in which mental defect occurred it was present in thirty-one cases in both members, and in thirty cases was of a similar kind. In thirty-two pairs of like-sexed fraternal twins, both were feeble-minded in fourteen cases; five were mentally defective in different ways, and one member was normal and the other feeble-minded in thirteen cases. Among the twins of unlike sex, seven had similar mental defects, four had dissimilar mental defects, and in fourteen pairs one was defective and the other normal. If further investigations confirm these extraordinary findings it will appear very probable that most mental deficiency is genetic in origin.

Insanity, like feeble-mindedness, is due to both hereditary and environmental causes. Like feeble-mindedness also it occurs in many varieties and degrees of manifestation. For these reasons and others besides, the genetics of insanity presents many baffling problems. But despite such difficulties, a good deal of light has recently been thrown on the role of heredity in several types of mental disorders. The environmental causes to which insanity has been attributed are many. Besides worry, depression, severe mental shock and physical injuries, insanity may result from several kinds of diseases. Chief among these is syphilis, which is now recognized as responsible for the disorder known as general paresis, or general paralysis of the insane. 
The disorder commonly terminates fatally and, according to Dr. Mott, 20 per cent of the deaths in the London County Asylum are due to this cause, besides " another 5 to Io per cent of cases of brain disease dying in asylums with softening of the brain."

It was formerly the fashion more than now to attribute a great deal of insanity to alcohol. Unquestionably excessive alcoholism sometimes leads to insanity, but its influence in this respect has often been exaggerated by zealous reformers. According to some alienists a role of great importance is played by focal infections. In a book on The Defective, the Delinquent, and the Insane, Dr. H. H. Cotton has reported a high percentage of cures following the removal of infected teeth, tonsils, or parts of the intestine of insane patients. These findings have not generally been confirmed by the experience of other alienists. The recovery of an insane patient after an operation is adduced as a proof that the insanity was not hereditary. But this conclusion is based upon a prevalent misconception of how heredity works. Many people have severe and long-standing focal infections without showing a trace of mental unsoundness. If infected teeth or tonsils occasion an attack of insanity, it is not unreasonable to suppose that an individual is already predisposed by virtue of his hereditary diathesis. The fact that a patient recovers after an aggravating condition is removed is no proof that a diathesis to mental disorders was not present. On the other hand, the presence of such a diathesis is not proved. Why focal infection should be the occasion of insanity in some people and not in others we do not know. All that can be said is that many analogies suggest the cooperation of the "insane diathesis."

The study of the genetic factors in insanity is made difficult by a number of circumstances. The classification of the various types of insanity is admittedly in a very unsatis- 


\section{I38 HUMAN GENETICS AND ITS SOCIAL IMPOR'T}

factory state. Several typical forms are distinguished, such as manic-depressive insanity, dementia praecox or schizophrenia, and others, but there are many intermediate and overlapping forms. It is inevitable that the particular symptoms of a mentally diseased individual should be greatly influenced not only by his physical condition, but by education, habits of life, emotional experiences, and the innumerable circumstances that have molded his personality. And to the many environmental influences must be added the varied effects of different combinations of genes, which

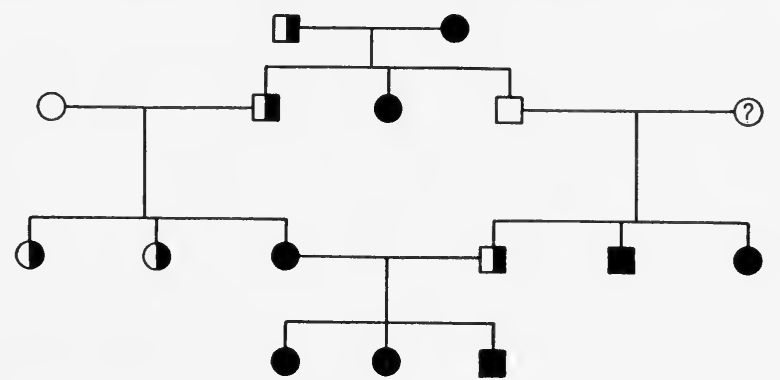

FIG. 54.-Pedigree of manic-depressive insanity. Milder cycloid cases indicated by half darkened circles or squares. (After Hoffmann.)

conspire to modify the operation of any gene defect that may be primarily responsible for the insane diathesis.

It is a well-established fact that the insanities which run in families usually conform to a particular type. Most kinds of hereditary insanity depend on recessive or partly recessive factors, and hence the majority of individuals who owe their insanity to heredity come from parents who are not insane. Out of $65 \mathrm{I}$ cases of manic-depressive insanity studied by Rüdin, about three-fourths came from parents both of whom were normal. In the siblings of these threefourths, only about 7 per cent had manic-depressive insanity. If, however, an individual with manic-depressive insanity had one parent with manic-depressive insanity, the same type of insanity was found in 24 per cent of his siblings. In 
similar studies of $72 \mathrm{I}$ patients with dementia praecox whose parents were free from this disorder, it was found that 4.5 per cent of the siblings were afflicted with dementia praecox; but if one parent and one child had it, 6 per cent of dementia praecox was found among the siblings. Hoffmann found among I54 children of dementia praecox parents thirteen to fifteen, or 8.6 to Io per cent were cases of the disorder. Both Rüdin and Hoffmann conclude that dementia praecox depends upon two pairs of genetic factors.

These authors found that among the brothers and sisters of individuals with dementia praecox a large percentage were mentally peculiar (schizoid) to a greater or less degree. It has been suggested that these schizoid individuals are heterozygous for the incompletely recessive factors upon which dementia praecox depends. Under favorable circumstances, some of these might topple over into the limbo of insanity. Not improbably if such individuals become insane they would be more amenable to cure.

There are other varieties of insanity in which the role of heredity is more obscure. In the type known as Huntington's chorea, however, heredity is apparently the all-important factor. This malady is inherited as a typical Mendelian dominant. Davenport and Muncey have worked out the family history of 962 cases who derived from six or seven ancestors including three brothers who came to the United States in the seventeenth century. The disease usually begins relatively late in life after the individual has married and had children. It starts as irregular muscular movements or tremors. Later the gait becomes unsteady, speech is impaired, the mental faculties deteriorate, and the symptoms finally lead to a fatal termination. It might be thought that the hereditary character of this fatal malady would be so generally recognized that it would lead to its disappearance, but considerations of heredity usually have little effect 
upon the matings of human beings. When the parents of Emma T. warned her that Jesse H., whom she wished to

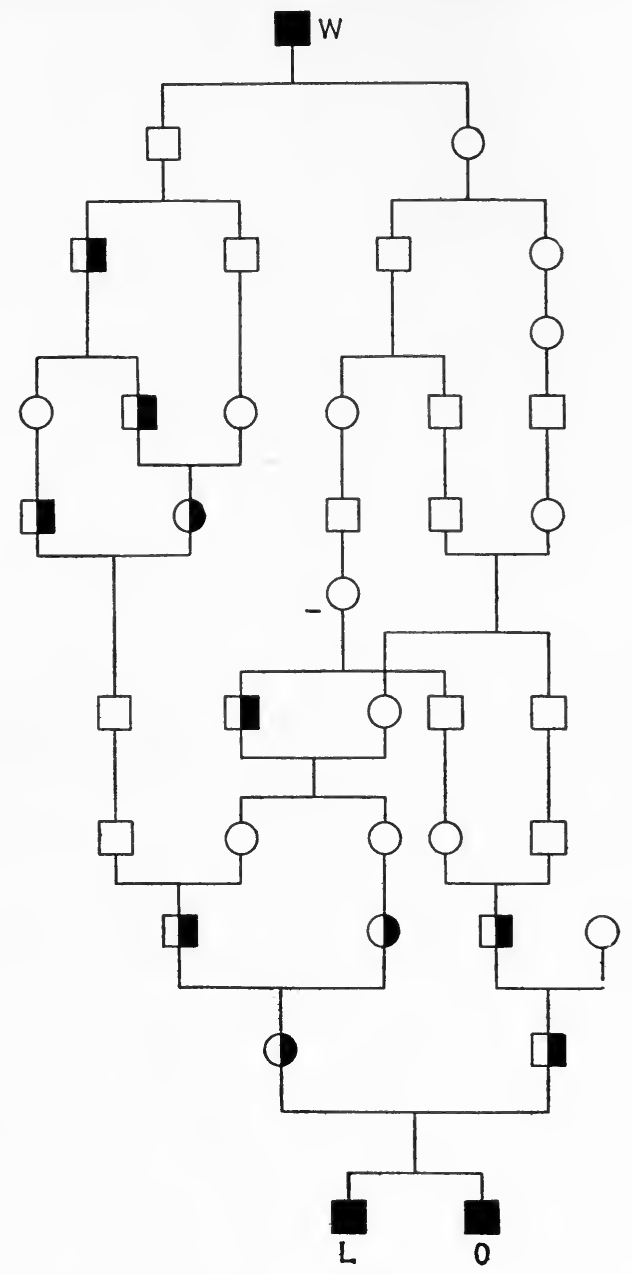

Fig. 55.-Pedigree of Ludwig II, $(L)$ and Otto I, $(O)$ of Bavaria who were descended from William of Braunschweig-Luneburg, $W$. All three were affected with dementia praecox. The half black squares or circles represent psychopathic individuals, mostly schizoid. Note that the two lines from the same ancestor come together in the parents of the two affected brothers. (Data from Strohmayer.)

marry, would probably become choreic because his mother suffered from this disorder, she replied that she would 
HEREDITY IN MENTAL DEFECT AND DISEASE I4I

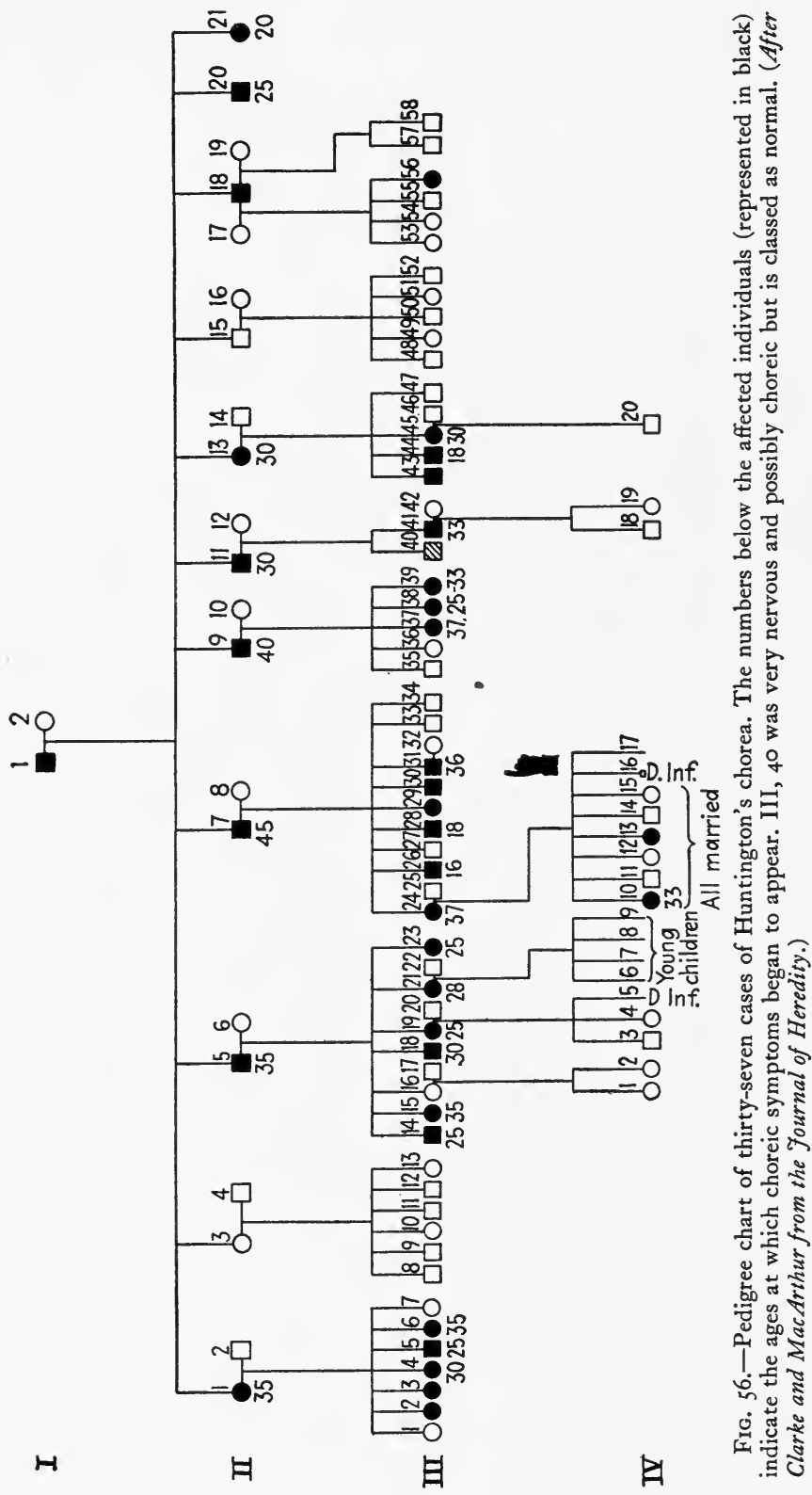




\section{I42 HUMAN GENETICS AND ITS SOCIAL IMPORT}

marry him anyway, so that she could take care of him. As events turned out, "She had to care not only for him, but also for four affected children."

The role of heredity in causing insanity is strikingly exemplified in the study of the mental disorders of identical and fraternal twins. Luxenburger has reported on the occurrence of dementia praecox in seventeen pairs of twins adjudged to be identical. In ten of these both members were clear cases of dementia praecox. There were three pairs in which one member had dementia praecox and the other twin probably had this same disorder. Only in two instances was one twin considered to be normal. With a larger series of fifty-six dementia praecox cases in twins, Lange finds that both twins had the disorder in fifty-two pairs. In four others one member was designated as schizoid. There was a pair in which one member was "abnormal," and one case in which the afflicted individual met with an injury to the skull.

Recently Rosanoff, Handy, and Plesset have reported on a study of ninety pairs of twins in which one or both members were affected with manic-depressive insanity. Of the twenty-three pairs of identical twins, both members were manic-depressives in sixteen cases and only one member of a pair was affected in seven cases. Of the sixty-seven pairs of non-identical twins, both members were affected in eleven cases and only one in fifty-six cases. It is apparent from such data that both heredity and environment play a part in causing manic-depressive insanity. It is very significant that in all the cases in which insanity occurs in both identical twins the disorder was of the manic-depressive type and never of different types, although in some cases the symptoms were rather different.

In a similar study of dementia praecox in twins the same authors found twenty-eight cases of probably identical 


\section{HEREDITY IN MENTAL DEFECT AND DISEASE I43}

twins in which both members were affected and thirteen in which the insanity was confined to one member of the pair. In nonidentical twins both were affected in fifteen cases and only one member in eighty-six cases. If we take the percentage of cases in which both twins are affected, we find that it is 68.3 per cent in the identicals and 14.9 per cent in the fraternals. The fact that in several cases of identical twins only one individual develops insanity indicates that heredity may not be the sole cause of the affliction. What is inherited is apparently a proclivity or diathesis to insanity which under certain conditions may fail to produce its dire effects.

Epilepsy, like insanity and feeble-mindedness, has long been observed to run in families to a certain extent, although it often appears quite sporadically for reasons that cannot be explained. There are many kinds of epilepsy, and unquestionably the disorder is sometimes caused by injury or disease. Some cases are attributed to head injuries at the time of birth. The prevalence of syphilis among epileptics is apparently considerably higher than in normal individuals, but the reported percentages of epileptics giving a positive Wassermann reaction are so exceedingly varied as to make the role of syphilis in the causation of epilepsy very uncertain. One relatively rare variety known as myoclonus epilepsy is the subject of a well-known memoir by Lundborg, who has traced all the seventeen known cases in Sweden to a single ancestor. The malady was transmitted like a single-factor recessive character. Owing to its recessive nature, the trait frequently appeared among the offspring of consanguineous marriages.

Inherited epilepsy usually behaves as a recessive or partly recessive trait, although Gordon has described a few families in which it seems to be an irregular dominant. In one family in which both parents were epileptic, one daughter was 


\section{I44 HUMAN GENETICS AND ITS SOCIAL IMPORT}

epileptic while the other was not. The epileptic daughter had four children of whom two had the malady. One of these produced two sons, both epileptics, while the other epileptic individual had a daughter so afflicted. One normal daughter married and produced three epileptic children. The daughter of the original pair that did not develop epilepsy married and had two children, one normal and one epileptic. The epileptic offspring married and produced four children, three of whom became afflicted with the same disorder.

Such a clustering of cases within a family can hardly be due to chance. It is likely that the different kinds of epilepsy

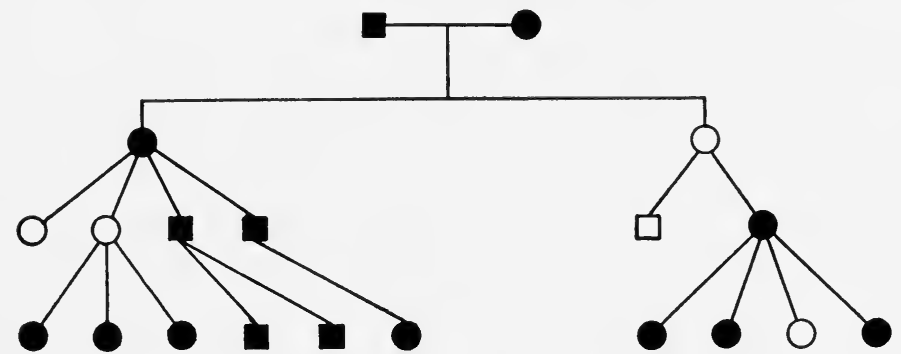

Fig. 57.-The inheritance of epilepsy through four generations. (After Gordon.)

are inherited in different ways. Owing to the fact that epilepsy is usually a recessive or partially recessive character of relatively rare occurrence, many cases of inherited epilepsy doubtless arise in families in which no other instances of its appearance have been observed. This circumstance has led many writers to minimize the role of heredity in the production of this disorder. As in other similar cases the study of twins has revealed the probable working of genetic factors that would not otherwise be evident. In four pairs of presumably monozygotic twins reported by Humm, epilepsy appeared in both members in three cases and in only one member in one case. In all the six pairs of dizygotic twins with epilepsy the malady was confined 
to one member of each pair. Olkon has described a pair of identical twin boys in which both were affected with a peculiar type of epilepsy beginning at an early age. Luxenburger has compiled records of epilepsy in seven pairs of probably identical twins and finds that in five pairs both members were similarly affected. Four additional pairs of twins with epilepsy have been reported from Holland by Dr. J. Sanders. In one pair, Mien and Nel, who were said to be monozygotic by the physician who attended their birth, both girls had their first attack of epilepsy in their fourth year within a few days of each other, and the later course of the seizures was much the same in both. In a pair of identical twin boys, Jan and Cor, epileptic attacks occurred in both boys before the end of their second year. The general character of the attacks, their premonitory symptoms, and after-effects were similar in both boys. The father also was subject to epileptic fits from his twelfth to his thirtieth year. In a third pair of twins both members had their first epileptic seizure on the same day, but one girl commonly had more severe symptoms than the other; and while both appeared at first to be about equally bright, the twin who was afflicted to the greater degree suffered more mental deterioration than her sister, so that there came to be a considerable disparity in their general intelligence. A fourth pair of twins described by Sanders consisted of a boy and a girl, both epileptic. Both had several convulsions early in life, and they continued to have similar epileptiform symptoms up to the time at which their case was described.

Recently Dr. K. Conrad has published a study of epilepsy (Rev. Neurologique, 64 , p. 485 , I935) in 258 twins. In the thirty pairs adjudged to be identical there were twenty-one concordant and nine discordant cases. It is an interesting fact that Conrad's study was presented before the Interna- 


\section{I46 HUMAN GENETICS AND ITS SOCIAL IMPORT}

tional Neurological Congress following a paper in which Prof. Abadie contended that epilepsy is a constitutional disorder in which heredity plays a very minor part. Instances of epilepsy in twins are as yet not very numerous, but the evidence thus far accumulated points strongly to the importance of genetic factors in causing this disorder, although they may of ten require the cooperation of some unfavorable environmental influence to enable them to become manifest.

The nervous system of man seems to be especially prone to hereditary ills, and we can mention only a few of the many types which have been described. One very unfortunate malady, Leber's atrophy of the optic nerves, which leads to more or less complete blindness, is commonly inherited as a recessive sex-linked character, appearing therefore, except rarely, only in males. In some cases, however, the defect is transmitted as a simple dominant. A relatively rare nervous disease, multiple neurofibromatosis, involving the formation of tumors on the nerve trunks, is usually transmitted as a dominant character, although it occasionally skips a generation. Another dominant defect frequently considered to be nervous in origin is progressive muscular atrophy. Macklin and Bowman described a family in which there were twenty-one affected individuals in three generations with atrophied peroneal and calf muscles of the lower part of the leg. In other families with inherited muscular atrophy, different sets of muscles, such as those of the arms, trunk, or face are apt to atrophied. Other defects produce hereditary tremors, and a peculiar rare disorder which causes individuals to move both their hands in the same manner (bimanual synergia) is characteristic of certain families. Some of the numerous hereditary disorders of the nervous system are manifested by very varied symptoms, while others are remarkably specific. Little is known of the developmental physiology of these defects. 
Why some gene changes cause the optic nerve to atrophy, while others lead to the development of tumors on the sheaths of nerves or give rise to dementia praecox or the atrophy of certain motor ganglion cells of the spinal cord, we can only vaguely conjecture. Human heredity involves the cooperative activities of hundreds and probably thousands of genes each of which plays its part in the development of the body. There are hundreds if not thousands of ways in which development may go wrong as a consequence of the modification of particular genes. And doubtless many new gene mutations will arise which will result in hereditary defects never heard of before.

\section{Suggested Readings}

Gates ('29), chap. 15. Holmes ('21) chap. 3, ('33), chap. 2. For further information see Barr ('29), Baur, Fischer, Lenz ('31), Doll et al. ('32), Goddard ('14), Myreson ('25), Rosanoff et al. ('34), ('35), Tredgold ('30).

\section{Questions}

I. It was formerly claimed that insanity tends in the course of generations to become transformed into imbecility and finally to extinguish itself. Is this probable from the standpoint of genetics?

2. What are some of the chief difficulties in determining Mendelian ratios in hereditary forms of insanity?

3. If you wished to ascertain whether insanity tends to be associated with genius, how would you investigate the problem and what sources of error should be guarded against?

4. Can most light be thrown on the inheritance of insanity through working out coefficients of correlation between relatives or by the intensive study of pedigrees?

5. What are some of the chief varieties of amentia?

6. What can you find out concerning the condition of the brain in amentia? in insanity?

7. Is the circumstance that Mongolian idiocy is especially prone to occur in the last member of a series of children a conclusive proof that particular genetic factors are not required for its production?

8. If a pair of identical twins were heterozygous for a factor tending to cause insanity, would one member be more apt to be sane and the other 


\section{48 HUMAN GENETICS AND ITS SOCIAL IMPORT}

insane than if both twins were homozygous? What analogy might there be between this case and the appearance of abnormal abdomen in Drosophila?

9. Suppose it were possible through the proper nurture to prevent the development of all cases of dementia praecox. What bearing would this fact have on the genetics of this malady? Consider again the analogy with abnormal abdomen in Drosophila.

10. What facts indicate that some forms of mental defect and insanity are caused by partially dominant factors?

II. Why are some forms of mental deficiency and epilepsy unusually common in inbred communities? 


\section{CHAPTER XII}

\section{NATURE AND NURTURE IN MENTAL DEVELOPMENT}

7 HAT some people are naturally bright and others 1 naturally stupid is a matter of common observation. Are the mental differences between individuals determined mainly by their peculiar hereditary endowments or by their environments and educational opportunities? All except a few extremists concede that these differences may be due to both hereditary and environmental influences, but opinions diverge greatly as to which of these factors plays the predominant role. The question is of crucial importance for both theoretical and practical eugenics. We find learned and able representatives on both sides of this question, and it will continue to be a fruitful subject of controversy until the results of investigation furnish conclusive proofs in support of the one or the other position.

In considering this problem one should endeavor to maintain a judicial instead of a partisan attitude, weighing the arguments pro and con in the endeavor to form the best judgment as to which position is favored by the preponderance of the evidence. In a large proportion of the questions which one encounters in the social sciences, a conclusive answer one way or the other cannot be given. One has, therefore, to make the best judgment possible. One may be persuaded as to the correctness of a certain standpoint and cling to his convictions until absolutely compelled to abandon them, but this is the attitude of a pleader rather than an impartial judge calmly endeavoring to discover which view is most probably right. 


\section{I50 HUMAN GENETICS AND ITS SOCIAL IMPORT}

Attitudes on the nature-nurture problem are very apt to be determined by the way in which the answer might affect other beliefs to which a person is committed. There is a strong bias in favor of the natural equality of man and consequently a reluctance to admit that inequalities of mental ability are determined by heredity. Social reformers are often loath to admit that inferior mental development may be due to the germ plasm, because they prefer to regard it as resulting from remediable causes that can be largely overcome through their beneficent ministrations. Religion, economic theories, race prejudice, and other factors are potent influences in determining attitudes on our problem, as a perusal of the controversial literature on the subject will make apparent.

In endeavoring to arrive at the most probable conclusion on this controverted topic, one should consider it broadly from the standpoint of the antecedent probabilities of the case. At the same time, one should keep clearly in mind that it is unsafe to attempt to settle the question from this standpoint alone. Whatever may be the precise way in which mind and body are related, there can be no question as to their very intimate association. As the body develops from an embryo through the stages of infancy and childhood to maturity the mind undergoes parallel changes. During the long course of evolution body and mind have evolved together. The mental qualities of different species of animals vary enormously, and unless variations in mental endowments were inherited it is difficult to see how the minds of animals should differ so greatly, or how the mental powers of man could have come to be so superior to those of other animals. In the individual person, influences affecting the brain have an effect also upon the mind. Brain injury through accident or disease leads to mental impairment, and the recovery of mental health often follows upon the restoration of the normal physical condition. 
All this shows that mind and body manifest a strong tendency to vary together. If bodily variations are due to genes, as they manifestly are, one would expect, therefore, that mental changes would be effected through the same cause. The recessive gene productive of amaurotic idiocy not only causes severe physical disabilities, which result in early death, but it produces an impairment of mental development, resulting in a low order of imbecility. The dominant gene that is responsible for Huntington's chorea exercises its baneful influence at a much later period of life, but its physical symptoms are accompanied by a grave impairment of mentality. One can draw no hard and fast distinction between physical and mental characters. So many physical disorders, both hereditary and acquired, have their mental symptoms that any discussion of the inheritance of mental as distinct from physical traits is futile. Emotional peculiarities are demonstrably dependent upon the development of the endocrine glands, and the latter vary, like other organ systems of the body, as a result of differences in hereditary factors. If one were to maintain that mental qualities are unaffected by the inherited constitution of the body, it could be only on the basis of some peculiar theory of the relation of mind and body for which it might be difficult to find adequate support.

In a previous chapter, evidence has been adduced in support of the view that mental defects and diseases are, at least to a certain degree, the product of defective genes. If the lower grades of mental development may result from poor germ plasm, it would seem not unlikely that differences in genetic constitution might occasion varied intellectual endowments among normal and superior individuals. Consider the structural and physiological peculiarities among your normal friends and acquaintances, with their differences in stature, weight, bodily form, shape of head, facial expression, and numerous other characteristics resulting from their 


\section{I52 HUMAN GENETICS AND ITS SOCIAL IMPORT}

varied combinations of genes, and in the light of what we know of the relation between physical and mental traits, it will hardly seem probable that the latter are not affected by these variations of hereditary composition.

Few, however, would deny that hereditary influences exert some effect on the development of the mind. Most environmentalists maintain that such influences are less potent in determining mental development than the influence of varied environments and cultural advantages. We have here a problem of the relative influence of two factors, the potency of each of which is conceded by all but extremists of both camps. It is, of course, possible that the answer to the question may differ according to whether we are dealing with mentally defective and diseased individuals or with people in the higher intellectual levels. In such types of mental defect as that followed by Sjögren in an inbred community in Sweden the case for the hereditarian is quite clear. That the superiority of Shakespeare or Newton is due more to a fortunate allotment of genes than the influence of favorable nurture is a very different problem.

Genius is often regarded as a mysterious and unaccountable phenomenon appearing somehow quite regardless of parentage. The first effectually to controvert this notion was Francis Galton, whose work on Hereditary Genius published in I869 showed that superior mental ability has a marked tendency to run in families. This conclusion was supported, not by amassing cases that happened to bear out the thesis, but by compiling lists of the most eminent persons and making a study of their near relatives. It was found that eminent people have eminent near relatives many times as frequently as do ordinary, undistinguished individuals. Moreover, the chance that the relative of a great man will also become great was found to decrease with the remoteness of the relationship. Additional support for Galton's conclusion was furnished by 
a later work on English Men of Science and in a volume written in collaboration with Dr. E. Schuster on Noteworthy Families.

In the earlier studies on the inheritance of mental abilities, reputation for distinguished achievement was used as a measure of intelligence. Later, use was made of the scholastic records of related individuals in schools and colleges. Pearson and his coworkers have shown that siblings resemble one another in scholastic tests to about the same degree that they

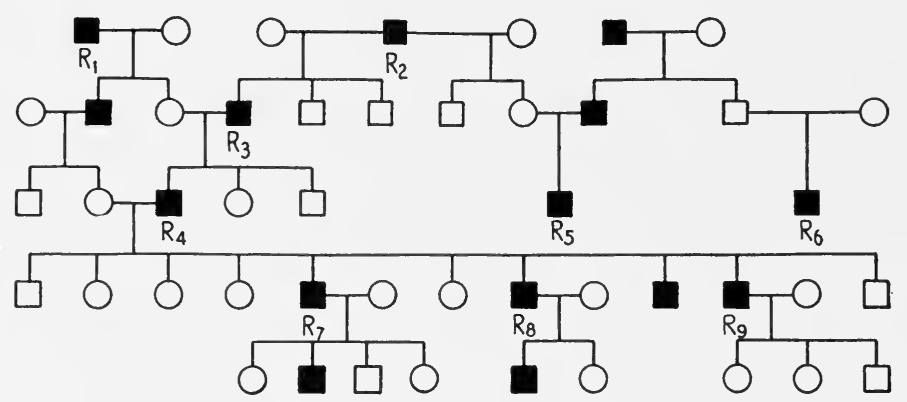

Fig. 58.-Pedigree of the Darwin family. Black squares represent men distinguished for intellectual achievements. $R$, members of the Royal Society; $R_{1}$, Josiah Wedgwood, F.R.S., founder of the Wedgwood pottery works; $R_{2}$, Erasmus Darwin, celebrated physician, scientist, and proponent of evolution in the eighteenth century; $R_{4}$, Charles Darwin; $R_{5}$, Francis Galton; $R_{7}$, George Darwin, astronomer and mathematician; $R_{3}$, Francis Darwin, Professor of Botany in the University of Cambridge. The fourth son of Charles Darwin, Leonard Darwin, was president of the first two international congresses of eugenics, author of works on eugenics, and president of the Geographical Society. The son of George Darwin, C. G. Darwin, is a prominent physicist.

do in various physical traits. Correlations between parents and offspring for scholarship based on records of fathers and sons attending Oxford University showed a fairly high positive correlation (about 0.3 ), although less than that between brothers (0.5). This, it was inferred, was because methods of instruction and standards of grading, etc., were more similar for brothers than for fathers and sons.

When mental tests came in to vogue, numerous studies were made on the scores of children of the same family. The studies of Pintner on 180 pairs of siblings gave a correlation of 0.39 , those of Rensch on 365 pairs, a correlation of 0.45 , 


\section{I54 HUMAN GENETICS AND ITS SOCIAL IMPORT}

and those of Willcocks on 365 pairs in different environments a correlation of 0.5 . These results are typical of many others, so one may infer that insofar as intelligence is measured by these tests it runs in families to a very considerable extent.

Such findings, however, do not convince the environmentalist that the familial distribution of intelligence is due to heredity. He would explain it as due to the unequal distribution of educational opportunity and incentive in different families. Demonstrably the ability to pass an intelligence test is in part due to previous training. Given two persons of the same hereditary endowment one of whom has had no educational advantages, while the other has been engaged in intellectual pursuits that have exercised his mind upon a number of different problems, it is evident that in almost any kind of test devised the two individuals would make quite different scores. Even when efforts are made to eliminate the influence of mere information in passing intelligence tests it is not possible to get rid of the influence of scholastic experience. No test measures innate ability. What is measured in every case is a product of both heredity and experience with the environment. One may explain the fact that Mary is more intelligent than Lucy in either one of two ways. If it is said that Mary's parents were more intelligent than Lucy's, one might reply that Mary's parents had the better educational opportunities, and that therefore heredity has had nothing to do in making the one girl more intelligent than the other. Two people might argue the question indefinitely without convincing either party. According to some writers the question of the relative influence of nature and nurture on mental development has come to a practical impasse. The problem is a general one which, as we have seen, is encountered in many relations. Where it is presented in lower organisms, which can be subjected to controlled experiments, it is possible to 
arrive at a decisive answer. Could we expose people with the same heredity to different conditions of the environment, we might obtain an accurate measure of the various kinds of environmental influence. If, on the other hand, we could keep the environment just the same for persons of different heredity, we might obtain a fair measure of the extent to which heredity is responsible for their differences. Unfortunately for the solution of our problem, we cannot subject human beings to the kind of experimentation that would be ideally most desirable. Moreover, in most cases it is impossible absolutely to rule out the influence of either hereditary or environmental differences. Nevertheless, there are ways of studying these effects and of gaining some measure of their extent.

In considering the influence of environment upon intelligence, we should distinguish between two classes of effects: (I), the stimulation of development beyond the normal standard, and (2), the thwarting or retardation of normal development. There seems to be no limit to the latter kind of influence short of causing the death of the individual. It has been shown that, in general, children with many physical defects have also a low mentality. Ayres found that out of 3,304 children in New York those rated as dull had a higher percentage of physical defects than those rated as bright. This may be due not to the fact that physical defects cause mental retardation, but because both kinds of defects result from a common cause either in bad heredity or in an unfavorable environment. There has been some concern over the possible influence of malnutrition on mental development because during the great war many children suffered much from lack of adequate food. According to the investigations of Blanton, the permanent effects of malnutrition were relatively slight. Several experimental studies have shown that when undernourished children 


\section{I56 HUMAN GENETICS AND ITS SOCIAL IMPORT}

are given proper food their physical condition improves, but there is no significant change in their I.Q's. There is also little relationship between diseased tonsils or adenoids and mental development, nor is removal of the offending organs followed in general with an increase of intelligence. Mental retardation in schools has been found by Smillie and Spencer to increase with severity of infection with hookworm, but this may be due to the fact that the more heavily infected children come from poorer types of homes which are associated with the low mental status of the parents.

As we have already pointed out, there are several diseases which sometimes lead to an impairment of mental development. Pintner found that children who are congenitally deaf are mentally retarded by two or three years. Among the children whose deafness was acquired, the later the onset of deafness, the higher the average I.Q. of the children. The lowering of the I.Q. in deaf children is probably to be ascribed not only to the effect of the deafness per se, but to the influence of the cause of deafness (meningitis, syphilis, and other diseases) which have a certain tendency to produce mental retardation.

Aside from the effects of disease, inequalities of mental development may be produced by intrauterine causes, birth injuries, alcoholism, drug addiction, and the various agencies adversely affecting the health of the individual. In exceptional cases injury or disease may make all the difference between an intelligent person and a low grade imbecile, but the extent to which repressive factors are responsible for the mental differences commonly observed among human beings cannot be precisely ascertained. More commonly differences in intelligence are attributed to training, but in order to ascertain whether or not this view is correct we need to know something of the possibilities and the limitations of training in promoting intellectual 
development. By feeding his animals in different ways, a farmer may cause some of them to become mere runts and others to grow beyond the average size, but no amount or kind of food will cause his sheep to become as large as cattle or even make his Bantam chickens attain the size of Plymouth Rocks. So far as physical growth is concerned, environment has its very distinct limitations. Is this true also for the development of the mind? Most of us can recall many individuals, some of whom we have known from our early school days, who, we are quite sure, would never blossom out into great intellectual leaders in any imaginable environment. Even in the absence of any scientific tests of intelligence it is apparent that in most individuals the capacity for very distinguished intellectual achievement is not there. Education may do much for people. For some people it can do much more than for others. For idiots and imbeciles it can do relatively little. For borderline or dull-normal people, it can do more, but can it cause them to get over their dullness? A Pascal or a Macaulay may astonish the world with the extent of his early acquisition of knowledge. Apparently the more one is favored by hereditary gifts the more he will profit from his favorable opportunities. If Macaulay had been brought up in ignorance on a desert island doubtless he would never have become famous. If, unknown to the parents, another boy had been substituted and reared in the same environment, would he have exhibited Macaulay's phenomenal memory and powers of understanding? As a matter of fact, people show varying degrees of capacity for intellectual development. Even the best of all pedagogical procedures cannot make a genius out of a dull boy. As a rule intellectual capacity is exhibited early and is commonly retained throughout life, although not infrequently there are individuals who do not justify their early promise. The studies of Cox on the boy- 


\section{58 HUMAN GENETICS AND ITS SOCIAL IMPOR'T}

hood of great men have brought out the fact that, contrary to a rather prevalent opinion, great men exhibit as children an unusual degree of precocity.

In an investigation more or less complementary to that carried on by Cox, Dr. Terman has studied the home life, preschool training, physical development, temperament, and other peculiarities of a thousand children in California schools who made a grade of $\mathrm{I}_{4} \mathrm{O}$ or more in an intelligence test at the beginning of the study. The grade $\mathrm{I}_{4} \mathrm{O}$ is an exceptionally high one, since it is reached by less than one-half of I per cent of school children. These gifted children were not abnormal or freakish, as prodigies are commonly considered to be, but on the whole they proved to be a normal, healthy, socially adaptable group of youngsters, keenly interested in sports and other nonscholastic activities. In school they received no more attention (in fact rather less) than the rank and file of pupils. Their preschool advantages were probably somewhat better than those of the average student, and many of them gave evidence of unusual intellectual interest before they entered school.

Several years later these same students were studied again. In the more advanced grades of school and in college, their records were high. Phi Beta Kappa and the other honors at graduation were obtained three times as often as by college students in general. "Nearly three-fourths of the total marks earned in High School by gifted girls and nearly one-half of those earned by gifted boys are of the A grade." There were a few irregularities of behavior of course; one boy from a broken home committed suicide, but as a rule the infractions of the moral code were not serious. Judged by their development thus far these children have abundantly justified their early promise.

Since these children constitute an unusually intelligent group, it is of interest to learn something of their ancestry. 
To a large extent the fathers of these children came from the professional classes or were engaged in pursuits requiring an unusual degree of knowledge or skill. It is a striking fact that nearly one-fourth of the names in the Hall of Fame were found in the ancestry of this group.

A strict environmentalist would attribute the persistent superiority of these gifted children to the advantages of their home life, or possibly more subtle influences, but before one is justified in adopting this position, it should be shown that a favorable environment is at least capable of producing the effects ascribed to it. If it were possible to rear a large random sample of children from infancy amid surroundings which are highly favorable for their intellectual development would these children show an average I.Q. of $I_{4} \mathrm{O}$ ? It may be said that we cannot tell because the experiment has not been tried, but many other experiments have been tried whose results have an important bearing on the question. If we could make exceptionally intelligent people out of children of ordinary heredity by exposing them to the proper kind of nurture the possibilities in the way of education would exceed the most sanguine dreams of the most optimistic educator. But what are the facts? If we select a fair sample of children whose I.Q.'s have been fairly tested, how much is the best type of training that has been devised capable of raising their level of intelligence? Is it 5, I0, 20, 50 , or 100 or more points? Were the average improvability shown to be very great, say 100 or more points, the environmentalists might well argue that training can account for all the intellectual differences among human beings, barring the effect of a certain amount of environmentally caused retardation. This would not prove that these differences actually were the result of environment and training, but only that they might be. On the other hand, if in no case training was proved capable of raising the I.Q. of children 


\section{I60 HUMAN GENETICS AND ITS SOCIAL IMPORT}

as much as Io points, the hereditarian might well argue that most of the differences in I.Q. were probably due to genetic factors, especially in view of the fact that both high and low I.Q.'s tend to run in families. Whatever may be said of possibilities, the results of retests on children exposed to different environments and methods of instruction, while they have indicated a certain amount of improvement in I.Q. under more favorable conditions of nurture, have not demonstrated any very striking increase in intellectual power.

There have been several studies on the results of transferring children from bad home environments to better homes or institutions, but for the most part this has not resulted in any marked improvement of the I.Q. Freeman finds that children adopted into better homes have a higher I.Q. than those adopted into poorer homes. The correlation between grade of home and I.Q. of adopted children was $0.52 \pm 0.06$. There was a certain amount of selective placement calculated as $0.34 \pm 0.07$ at the time of adoption, so that a part of the relationship between the intelligence of the foster children and the character of the home was due to the original selection of more intelligent children for the better homes. The higher I.Q.'s of the children in these homes is, according to Freeman, due in a considerable measure to their environment.

In a study of adopted children in California made by Miss Burks the observations were made on children adopted soon after birth, so that opportunities for selective placement on the basis of intelligence were practically eliminated. The families of the foster parents were a little superior to the average. The average I.Q.'s of their own children was II 5.4; those of the foster children, 107.4. The correlation between the I.Q. of adopted children and the quality of the home was $0.35 \pm 0.05$. It is of interest that in the superior 
home, the range of variability as well as the average I.Q. was increased, being from 40 to 160 . In the fairly similar environments of superior homes some influences, which the hereditarian would say were the genes, evidently were responsible for enormous differences of measured intelligence. Miss Burks' conclusion was that "home environment contributes about I7 per cent variance in I.Q.'s; . . . the total contribution of heredity (i.e., of innate and heritable factors) is probably not far from 75 to 80 per cent."

If environment affords the chief factor in determining the differences between human beings, we should expect that children reared from an early age under very similar conditions would show a much greater similarity in intelligence than children in general. Miss Lawrence finds that children reared in an institution since infancy are about as variable in intelligence as are children of corresponding ages in the public schools. Davis found that the degree of mental similarity between children in an orphanage where conditions of nurture are relatively uniform showed no relationship to length of sojourn in the institution. Uniformity of nurture, in so far as this can be secured, does not result in any marked similarity in intellectual development.

In recent years a great deal of material of much interest to eugenists has been accumulated on the relation between occupation and intelligence. When the army mental tests were made data were collected on the I.Q.'s of different occupational as well as national groups. As might be inferred, members of the professions-doctors, lawyers, accountants, and especially engineers-tested high. Those in clerical work occupied a somewhat lower rank. Then came various classes of skilled workers. Those who made the lowest scores were miners, teamsters, and unskilled laborers. Since the degree of education varied in much the same order as the intelligence quotients, the question arose as to how 


\section{I62 HUMAN GENETICS AND ITS SOCIAL IMPORT}

far these results are indicative of differences in native intelligence.

It is not often that opportunities for testing the intelligence of a large general sample of the adult population are presented. There are in fact, relatively few studies on adult intelligence available, but children in schools afford much more readily measurable material for the mental testers, and millions of school children have been subjected

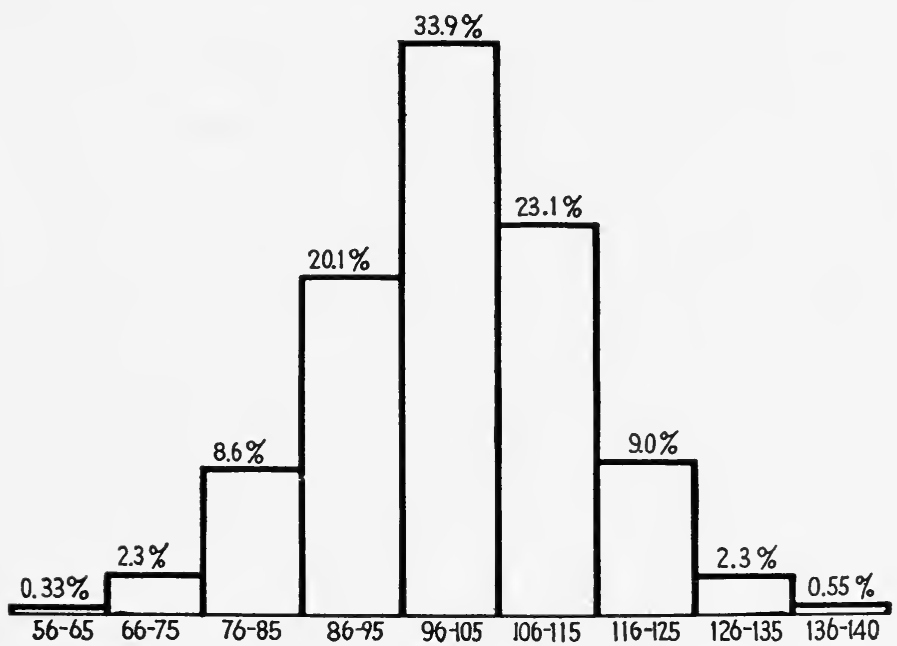

Fig. 59.-Distribution of the intelligence quotients of 905 pupils according to Terman.

to intelligence tests of all sorts. Several studies have been made on the I.Q.'s, as well as the scholastic grades of students in relation to the occupations of their parents. Quite uniformly the children of parents in the professional groups make the highest average scores. The children of unskilled laborers show the lowest I.Q.'s, and those of the intermediate occupational groups make intermediate records. The I.Q.'s of children show much the same gradation in relation to occupation as those of the fathers. Why? The position of the hereditarian is that only individuals of superior intelligence get into the professions, whereas, 
barring exceptional cases, the lower occupational classes on the whole include relatively more individuals in the lower intellectual levels. The environmentalists would explain the lower I.Q.'s of the unskilled laborers' children as the result of the poorer advantages afforded by the homes from which they came. Sometimes a very bright child comes from a home of lowly status, a fact which the hereditarian would attribute to good hereditary material in a low social and economical level. Who is right? Or are both right to a certain extent?

Some interesting data on this question have been supplied by Miss E. M. Lawrence from her studies of the I.Q.'s of over 380 illegitimate children who had been reared in an institution. The children had practically no contact with their fathers, and they were all less than one year old when they were given up by their mothers. The environmental conditions of the children were favorable and uniform. Information was collected on the occupation of the parents, and the I.Q.'s of the children were found to have much the same relation to the father's occupation as was shown by children in the public schools of London, although the differences between the classes were not quite so marked. Miss Lawrence concludes that the correlation between intelligence of children and social status of the parents "is not mainly due to the direct social influence of the home, but is a genuinely biological fact."

In another group of orphan children Jones and CarrSaunders have studied the relation between the I.Q.'s of the children and the occupations of their fathers. Here much the same gradation was found as in children in the public schools. Children from the lower occupational classes showed a slight average gain (about two points in I.Q.'s) after a few years of residence, while those of the higher groups showed a drop of nearly one point. There was thus a 


\section{I64 HUMAN GENETICS AND ITS SOCIAL IMPORT}

slight tendency for differences in I.Q.'s to decrease with longer residence in the institution, but the decrease was so small as to be within the probable error of measurement. 'The authors conclude: "There is no reason to suppose, so far as this evidence is concerned, that environmental influences are the whole or even the major part of the differences in intelligence between children of different social origin." At least if environment caused these differences it must have done so before the children were placed in the institution.

Miss Alice M. Leahy recently made a study of the I.Q.'s of foster children in Minnesota who were placed in homes before they were six months of age. Mental tests taken later showed that the intelligence of the children correlated closely with the occupational grouping of the parents and but slightly with the intelligence of the foster parents. The influence of the parents who supplied the heredity was apparently more potent than the influence of the parents who constituted an important factor of the environment.

As in other aspects of the nature-nurture problem, many significant bits of evidence on our problem are furnished by the study of twins. Since identical twins have the same heredity, whatever differences they may exhibit in mental traits must be attributed to environmental causes. Differences between fraternal twins are due to the combined influence of heredity and environment, and the hereditary differences may vary all the way from complete identity to those which cause one individual to become a genius and the other an idiot. Several studies have been made of the relative degrees of resemblance in the mental traits of identical and fraternal twins, and while the different investigations are not in entire agreement, they show that in general the I.Q.'s of identical twins differ much less than 
those of fraternal twins whether of the same or of opposite sex. According to Holzinger's studies on fifty pairs of identical twins and fifty-two pairs of like-sexed fraternal twins, the average intrapair difference in mental age was 8.4 for the identicals and 15.9 for the fraternals.

Wingfield in studying the correlations between twins from the public schools of Canada found a correlation (corrected for age) of $0.9 \mathrm{I}$ for identical pairs; 0.83 for like-sexed pairs; 0.72 for fraternal pairs; and 0.62 for unlikesexed pairs. Hirsch found the correlation between the I.Q.'s of thirty-eight pairs of similar twins to be $0.97 \pm 0.012$, a surprisingly high correlation, while between dissimilar twins it was $0.53 \pm 0.071$.

It is sometimes assumed that if, on the average, the I.Q.'s of identical twins differ by 5 points, while those of a comparable group of fraternal twins differ by ro points, the influence of nurture (assumed to be 5 points) and that of nature (Io - 5) would be practically the same. But would this conclusion follow? In all measurements there is a certain amount of variation to be attributed to errors of measurement. What is measured in all of these studies is ability to pass a certain type of intelligence test, whatever this may imply as to general intelligence. Two persons may have exactly the same heredity and exactly the same acquired ability to pass a given test, but their scores would doubtless not be identical. A certain amount of variability might be due to the person who scored the test. Another element is luck in answering questions. If two persons knew how to spell $95^{\circ}$ out of a lot of I,000 words and each was asked to spell 100 words chosen at random from the list, one person might be fortunate enough to make a perfect score, and the other might be unlucky enough to make 50 per cent of errors. To the extent that an element of sheer luck is involved in a test, to that extent variations in scores 
are not indicative of any variations in real ability either inherited or acquired.

Other disturbing factors are contributed by purely accidental happenings that may influence the performance of one or the other individual. Something eaten for breakfast that did not agree with one twin might cause him to fall short of giving an adequate account of his real ability. The results of every kind of test are due to causes of variance that to a certain extent have no relation to the real capacity or acquirements of the individual tested. How much of the difference in scores between identical twins should be attributed to this nonsignificant kind of variability, and how much is due to differences in knowledge or capacity we do not know. Most experienced mental examiners would concede that tests fall short of ideal accuracy by a few points at least. If such variance as we have discussed is as much as 5 points (which is a very conservative estimate) the average difference of 8.4 reported by Holzinger for identical twins would be mostly nonsignificant. The true difference might be only about 3 or 4 instead of 8.4 between identicals and somewhat less than 15.9 between fraternals, and thus the differences attributed to nature would be greater than is indicated by the uncorrected figures.

These considerations should be kept in mind in evaluating the results of measurements of the intelligence of identical twins reared apart as compared with the intelligence of identical twins reared together. Studies on identical twins reared apart should afford valuable information on the role of different environments in causing differences in intelligence. In some cases very remarkable similarities in mental score are reported in spite of differences in environment and educational advantages. In some other cases consistent differences in I.Q. are shown which exceed the average differences between fraternal twins. The average 
difference of ten sets of identical twins reared apart, who were studied by Newman, was 7.7 points; this is less than the average difference of fraternals reared together, which was 9.9. The average differences in scores of fifty pairs of identical twins reared together was 5.3 (Stanford-Binet test). The greatest difference in the I.Q.'s of identical twins reared apart was 17.7 points on the Stanford-Binet scale in favor of the city bred and better educated girl. This difference, which was paralleled by other mental tests, doubtless represents a real difference in intelligence, but whether it is due mainly to education or to factors in the nature of handicaps to development we do not know. Physically one twin, Mary, was about an inch shorter and was about a size smaller in most physical measurements.

Unfortunately, cases of identical twins reared apart who have been subject to mental measurement are as yet few in number, so that we know little of their normal range of mental differences. In general, such twins are much more nearly alike than ordinary individuals of the same age, sex, and socioeconomic status and are even more similar than fraternal twins reared together, a part of whose similarity is due to their common parentage. It should be borne in mind, however, that separation does not necessarily involve marked differences in environmental influence, nor does living in the same household necessarily mean exposure to similar influences on mental development.

One case of identical twins described by Gesell is especially noteworthy on account of the very superior intelligence shown by both members. Both girls began to talk and to walk when eleven months old. Before they were four years old they were able to read in English, French, and Esperanto. "Formal arithmetic was begun at six and in less than a year they were solving mentally problems in fractions and percentage. At the age of nine both were doing Junior 
High School work. They speak French fluently, and have made progress in Italian and have embarked upon Russian. They are much alike in tastes and dispositions. Their mental tests and their vocabulary tests give almost the same score." Physically they are very similar in many ways, including the presence in both of a small pigmented mole a little above the outer corner of the mouth. That the pronounced mental superiority of these twins is due to heredity is indicated not only by their close similarity, but by their ancestry in that "scientific and linguistic ability of a high order and physical energy are some of the traits which are found in the two immediate generations." Doubtless these twins enjoyed unusual educational opportunities, but it is evident that ordinary children would not have profited by these opportunities to nearly so great an extent.

As to the precise way in which heredity functions in the transmission of intelligence, we have little knowledge. The doctrine that superior ability is a recessive unit character is probably no longer taken seriously. That intellectual development is dependent upon many genetic factors is indicated by the fact that it appears to vary quite continuously from the lowest to the highest level. If people were graded according to their levels of intelligence their variability could be expressed in a fairly normal frequency curve, similar in shape to the curves obtained by measuring their heights or weights. The great majority, as common observation discloses, cluster around mediocrity as might be expected according to the multiple-factor hypothesis.

But there are not only different degrees of intelligence, there are also different kinds. As a rule, when people are unusually proficient in one subject they excel also in others. Nevertheless, there are special aptitudes of many kinds and occasionally there are highly intelligent people who are unable to make normal progress in mathematics or languages. 
There is evidence that special abilities as well as general intelligence runs in families, but the proper evaluation of the hereditary factors involved is complicated by the coincident influence of environment and family tradition.

If we would sum up what appear to be reasonable conclusions in regard to the role of nature and nurture in mental development, the following would seem to be justifiable statements:

Both heredity and environment may cause very great differences in the mental development of human beings.

Hereditary factors can make the difference between a low grade idiot or imbecile and the native endowments of the highest type of genius.

Environment can depress or inhibit the development of the mind to any degree consistent with the maintenance of life.

To a certain extent nurture can increase intelligence as this trait is measured by any kind of test yet devised. Its power of doing so varies in different individuals, being very slight in idiots and imbeciles and becoming greater as hereditary capacity is increased.

The power of nurture to increase intelligence is limited. There is no known method of making superior intelligence out of poor hereditary material. What is known of the effects of training makes it improbable that nurture is capable of producing the very high quality of intelligence found in certain family strains. At the same time, without favorable nurture, the members of such families would probably not have become noteworthy for their achievements. The fact that both high and low ability runs in families is due to both hereditary and environmental factors.

Different occupational groups are characterized by different average levels of intelligence, although the extent to 


\section{I70 HUMAN GENETICS AND ITS SOCIAL IMPORT}

which the differences are due to genetic instead of environmental factors cannot at present be estimated with precision.

There is still room for reasonable differences of opinion as to the role of nature and nurture in causing unequal degrees of intellectual development.

\section{Suggested Readings}

Holmes ('21), chap. 5, ('23), chap. 6 ('30), ('33) chap. 3. Jennings ('30), chap. 7. Lorimer and Osborn ('34), chaps. 8-10. For further discussions see Burks ('28), Ellis ('26), Freeman ('28), Galton ('69), ('74), ('06), Lawrence ('31), Leahy ('35), Newman ('29-'32), Schwesinger ('33), Spearman ('27), Terman ('25), Freeman ('34).

\section{Questions}

I. If you were perfectly free to experiment with human beings in any way that was desired, what experiments would you devise to test the relative influence of nature and nurture in mental development?

2. If children are well fed and cared for and have normal playmates, would children under five years of age develop intellectually much more in the home of a great man than in the home of a day laborer? If so, what would be the chief influences involved?

3. What can you say of the influence of nature and nurture on the intellectual development of Abraham Lincoln?

4. What influences in addition to those mentioned are apt to affect the attitudes of people on the nature-nurture problem?

5. Are there noticeable differences in intelligence between the members of your family? If so can you account for these as a result of experience?

6. Does environment commonly have more influence on the development of character and temperament than upon the development of intelligence?

7. What are some families noted for outstanding ability in music, in mathematics, in science?

8. What is the most plausible explanation of the mental differences between the members of a pair of Siamese twins?

9. What reasons can you assign for the conclusion that superior intelligence is not determined by a single genetic factor?

Io. What is meant by the "constancy of the I.Q."?

II. If the differences between the I.Q's. of identical twins were no greater than the probable errors of measurement, what conclusion would you draw? 


\section{CHAPTER XIII}

\section{GENETIC FACTORS IN CRIME AND DELINQUENCY}

7 HE role of genetic factors in the causation of crime is 1 difficult to appraise. The kinds of acts which are branded as crimes vary from age to age and from country to country. Probably some kinds of heredity more than others predispose people to commit antisocial acts, but whether an individual actually becomes a criminal depends largely upon the accidents of his environment. Even the most exemplary person might have become a criminal under just the right combination of circumstances.

The problem of controlling crime has proved to be one of the most baffling with which civilized peoples and especially Americans have to deal. Society rids itself of some of its criminal members by putting them into prison or through the cheaper and more certain expedient of capital punishment. Prisons and reformatories, so called, may keep criminals out of the way for a time, but as agents of reformation they are usually worse than failures. Speaking of the influence of penitentiaries H. E. Barnes has remarked in a recent work, The Repression of Crime, "instead of reforming the criminal they are in reality institutions for the training of more efficient and determined criminals."

Since the volume of crime is enormously greater in some countries than in others, it is reasonable to seek for the explanation of this fact in the influence of the social environment instead of heredity. The theory that most criminals are born and not made is not generally regarded as tenable by modern students of criminology. Lombroso held that 


\section{HUMAN GENETICS AND ITS SOCIAL IMPORT}

there are certain individuals whose inherited constitution has destined them for a life of crime. As a result of measurements made upon numerous criminals Lombroso concluded that the born criminal is characterized by certain anthropological marks among which are prominent cheekbones, asymmetry of face, scanty beard, and various other anatomical peculiarities, both external and internal. According to Lombroso these peculiar features are due largely to atavism, or reversion to a primitive, brutish type of humanity.

The theories of Lombroso were put to a statistical test by Goring, who made careful anthropometric measurements of about three thousand inmates of English prisons. Most of the characters which Lombroso regarded as indicative of criminal propensities were found to be no more prevalent among English convicts than in the general population. Goring, therefore, came to the conclusion that "there is no such thing as an anthropological criminal type." Nevertheless, he held that criminals commonly have a bad heredity that is manifested more in intellectual deficiency and emotional abnormality than in measurable anatomical characters. In some respects, however, physical differences were found to occur between different classes of criminals. Murderers were larger and stronger than individuals convicted of burglary or petty thieving, who were as a rule physically rather undeveloped. On the whole, the work of Goring has been regarded as having given the coup de grâce to the claims of the anthropological school.

Recently Dr. Hooton has again attacked the subject of criminal anthropology and has reported upon the results of an extensive series of measurements on different classes of criminals. He finds that in the native-born American population, murderers are heavier, taller, larger chested, more hairy, and have greater head circumference than individuals in the general population. Other types of criminals he 
states have their typical anthropological characteristics also. "In the sum total of their metrical and proportional features," says Dr. Hooton, "the criminals are also distinct from the civilians. The differences consist principally of a marked inferiority of bodily dimensions on the part of criminals, but also include some striking deviations in proportion."

Only a preliminary announcement of Dr. Hooton's results has as yet been published. After explaining that he holds no brief for Lombroso, whose methods he concedes are open to severe criticism, Hooton remarks that "I am beginning to suspect that Lombroso, like Darwin, was right." Apparently, the last word on the significance of criminal anthropology has not yet been said.

After the conclusions of Lombroso on criminal anthropology had come to be more or less discredited, the distinguishing feature of criminals was sought rather in their low intelligence than their physical peculiarities. This was largely due to the techniques for mental testing which had been developed by the French psychologist Binet. Mental tests were given to large numbers of prisoners and juvenile delinquents, and the general verdict was that these offenders are characterized by a relatively high degree of mental deficiency. The statement of Dr. Fernald that "at least 25 per cent of the inmates of our penal institutions are feeble-minded" is typical of the results of many studies. The percentage of prisoners in England who are feebleminded was estimated by H. B. Donkin as 20 per cent. One of the most extensive studies on the intelligence of criminals has been carried out by Dr. W. P. Root, who has given intelligence tests to I,9I6 prisoners confined in the Western Penitentiary of Pennsylvania. The average I.Q. of all prisoners tested was 76.2 , a grade only a little above the level indicative of feeble-mindedness. The per- 


\section{I74 HUMAN GENETICS AND ITS SOCIAL IMPORT}

centages of prisoners in the several grades of intelligence were as follows:

Table 2.-Intelligence Quotients of the Inmates of Western Penitentiary, Pennsylvania

Per Cent

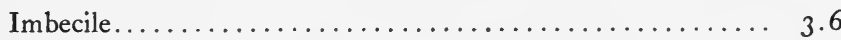

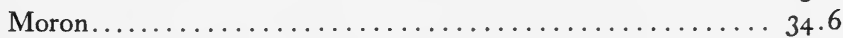

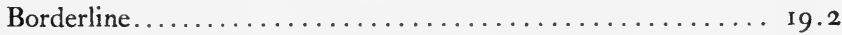

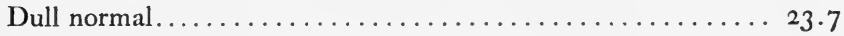

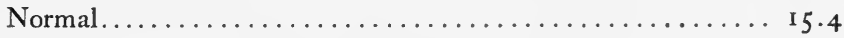

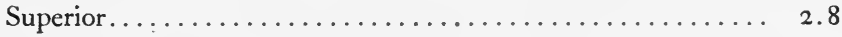

Very superior.................. 0.7

The I.Q. was found to vary considerably with the different types of crime. For embezzlers, it was high, 103.75; for homicides it was low, 70.9; and for burglars and robbers, it was 81.75 and 84.3 respectively.

It has been pointed out that in many studies of the intelligence of prisoners the criteria of feeble-mindedness used have been very unequal and are sometimes not stated at all. It has also been urged that most studies do not compare the intelligence of prisoners with that of the general population. Dr. Hermann Adler finds that the intelligence of criminals in several of the prisons in Illinois is about the same as that shown in the extensive mental measurements which were made in the United States army at the time of the World War. As a result of studies on the inmates of several prisons Dr. C. Murchison has come to the conclusion that the intelligence of criminals as a class compares favorably with that of the general population as revealed by the army mental tests. Unquestionably many of the studies on the intelligence of criminals are open to the criticisms which Murchison has directed against them. L. D. Zeleny has made an attempt to evaluate the diverse findings on the intelligence of prisoners by reducing the results of different studies to a common basis for comparison. It was found 
that the percentages rated as feeble-minded varied with the test used from 24. I to 36.7. Where mental age was used as a criterion, the ages selected varied from eight to thirteen years and the percentage of feeble-mindedness varied from 2.I to 47.3 ! Moreover, where comparisons with the general population have been made, the criteria of feeble-mindedness among the noncriminals have not been the same. Combining the statistical results of $I_{6} 6$ studies in which Binet tests were made on white prisoners, Zeleny finds that out of 6I,999 persons tested, I8,613, or 30.0 per cent, were adjudged mentally defective. By adopting as a criterion of feeblemindedness the mental age of eleven and reducing the results of all studies to the same standard, Zeleny finds that the percentage of mental defectives in the prison population as compared with that in the army draft is as $\mathrm{I} .8$ to $\mathrm{I}$.

Zeleny points out that in Murchison's studies the intelligence of native white prisoners was compared with that of the entire white draft, which included both native and foreign-born whites. Since the I.Q. of the latter was considerably lower than that of the former, the average does not give a fair basis of comparison. If the intelligence of the native born in prisons is compared with that of the native born in the draft, the percentage of mental defectives becomes changed from I 4.4 per cent in prisoners and 14.3 per cent in the draft to 17.8 per cent in prisoners and I 4.3 per cent in the draft. By applying the same standard to Adler's data, the proportion of defectives in the prisons of Illinois in relation to that in the $\mathrm{draft}$ is changed from approximate equality to $\mathrm{I} .22$ to $\mathrm{I}$.

It is generally conceded that prisoners may be somewhat less intelligent than criminals in general. The number of individuals convicted of almost inconceivably stupid crimes makes it likely that it is the less intelligent criminal who is 


\section{I76 HUMAN GENETICS AND ITS SOCIAL IMPORT}

most apt to be caught, whereas the embezzlers, swindlers, fraudulent promoters, and other high-class scoundrels usually succeed in evading the law. Persons of subnormal mentality are apt to be found in the lower economic levels and are hence more frequently tempted to commit crime under the pressure of want. Being less able to foresee the consequences of their conduct, they are more prone to act on the impulse of the moment, regardless of the result. Whatever relations may exist between mental defect and crime is probably indirect. The prison population is largely recruited from persons whose education is below the average as is clearly illustrated by the extensive statistical studies of Murchison and Root. For this reason, they tend to make a poorer showing in mental tests than their native ability would entitle them to.

How far the army draft ratings may be considered as representing the distribution of intelligence in the general population is open to question. Murchison admits that "it would seem reasonable to presume that the army norms are too low to be representative of the adult civil population of the country," but he contends that "the criminal norms are even more unrepresentative for the same reason!" The army tests served a useful purpose at the time by affording a rough classification of individuals according to their aptitudes, but there is strong evidence that education had a marked effect upon the scores that were made, since it is hardly credible that the native ability of the recruits from different states should differ as greatly as the records indicate. Hence these tests as a measure of native intelligence leave much to be desired. They afford about the only large sample of the adult population that has been subject to mental measurements. But they do not give us a very satisfactory basis for judging how the intelligence of prisoners compares with that of civilians. 
The intelligence of juvenile delinquents has been tested in a large number of groups. The general verdict of the testers is that subnormal intelligence is much more frequently found in delinquent boys and girls than in those who have kept out of trouble. Comparisons with normal individuals are more satisfactory than in the case of adult prisoners, because more adequate standards are furnished by the same type of mental tests applied to school children of corresponding ages. The percentages of delinquents classed as feeble-minded vary considerably in the different groups studied. This may be due to the kind of individuals designated as delinquents, and also to the standards employed in adjudging children to be feeble-minded.

One of the most extensive studies of juvenile delinquents has been carried out by Healy on four thousand repeated offenders in Chicago and Boston. Of these, I3.5 per cent were classed as "clearly feeble-minded." The standard of classification used is indicated by the remark concerning this group-" Practically all have an intelligence quotient of less than 75, and most of them of 70 or less." Of the offenders 72.5 per cent were rated as "definitely mentally normal."

Of I60 white boys admitted to the Lee School for delinquents in Philadelphia, A. A. Owens reports fifty-three, or 33.2 per cent, were ranked as morons having an I.Q. between 50 and $70 ; 35$ per cent were borderline cases, I 9.4 per cent dull normals, II.2 per cent normal, and only I.2 per cent with superior intelligence (I.Q. over IIO). According to the study of Mr. Cyril Burt on delinquent boys in England only 8 per cent were classed as feebleminded. Most of the studies on the intelligence of delinquent boys and girls indicate that the proportion of individuals of more than average intelligence is much smaller than in the general school population. 


\section{I78. HUMAN GENETICS AND ITS SOCIAL IMPORT}

It is scarcely needful to point out that many persons of defective intelligence are perfectly harmless and tractable creatures. Weak-minded girls, however, are notoriously prone to be led into sexual irregularities, because they are easily seduced by designing males. In our industrial society, prostitution is the natural fate of the unprotected feebleminded girl. It is not surprising that studies of the mentality of prostitutes should reveal a high percentage of subnormal intelligence.

Several writers have emphasized the importance of mental abnormality, rather than mental deficiency as a cause of crime. According to Lombroso and other criminologists, epilepsy is a factor of importance, especially in crimes of violence. Some of the most gruesome crimes have been committed under the influence of epileptiform seizures. Healy and Bronner find 5.5 per cent of epileptics among delinquents from Chicago, and I.6 per cent in those from Boston, the low percentage from the latter city being attributed to the fact that in Massachusetts there are special state institutions which care for a large part of the epileptic children. Inasmuch as epilepsy occurs in less than .3 per cent of the population, these percentages are significant.

A number of studies of prisoners have found over 50 per cent of the prison population to be in some respects mentally abnormal. The National Committee for Mental Hygiene has reported abnormal mental conditions found in jails and penitentiaries as shown in Table 3 .

Undoubtedly mental abnormality is responsible for many crimes. Persons who are unbalanced, like those with defective intelligence, find unusual difficulties in adjusting themselves to their social environment. Bad heredity is primarily responsible for many of these difficulties. The high percentage of delinquents coming from broken homes is to some extent indicative of temperamental peculiarities on 
the part of the parents. The bad environment which is so potent a factor in juvenile delinquency is often a product of bad heredity. But even if all bad heredity could be eliminated the problem of crime would still be with us.

Table 3.-Mental Abnormalities in Prisoners

\begin{tabular}{|c|c|c|}
\hline & Penitentiaries & County jails \\
\hline 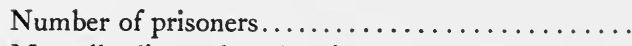 & $8,5^{81}$ & 3,206 \\
\hline Mentally diseased or deteriorated, per cent....... & $4 \cdot 9$ & $4 \cdot 9$ \\
\hline Epileptic, per cent................ & 0.7 & $1 \cdot 3$ \\
\hline Psychoneurotic, per cent......... & 1.2 & I. 2 \\
\hline Psychopathic, per cent......... & I 8.6 & $27 \cdot 4$ \\
\hline Mentally defective, per cent................ & I 2.5 & $13 \cdot 9$ \\
\hline Borderline, defective or subnormal, per cent...... & $14 \cdot 4$ & II.7 \\
\hline
\end{tabular}

Some rather striking evidence of the importance of genetic factors in the causation of crime has recently been brought to light through the study of the criminal careers of identical and fraternal twins. A few years ago Dr. J. Lange investigated all the twins in the prisons of Bavaria, together with a number of other pairs, and has embodied his findings in a small volume which has been translated into English under the title Crime as Destiny. By means of interviews, court and police records, conversations with relatives, and other methods of securing information, Lange succeeded in obtaining quite full histories of most of the individuals included in the investigation. Thirty pairs of twins were found one or both of whom had a prison record. Of these, thirteen pairs were identical and seventeen pairs fraternal. Lange states, "Among the thirteen monozygotic pairs the second twin was imprisoned in ten cases. Among the seventeen dizygotic pairs the second twin had been imprisoned in only two cases. This leads us to the following conclusion: as far as crime is concerned monozygotic twins on the whole react in a definitely similar manner, dizygotic twins behave quite differently. If, therefore, we attach importance to the 
twin method of investigation we must admit that so far as the causes of crime are concerned inherited tendencies play a preponderant part."

In commenting on these figures, Dr. J. B. S. Haldane, who has written a preface to the English translation of Lange's volume, remarks that "the odds that they are significant of a real difference are about seven thousand to one." Mere statistics, however, fail to give an adequate idea of the remarkable similarities in the careers of many of these identical twins; one needs to read the detailed histories as set down in Lange's book. In the identical twins studied there is a curious likeness in the types of crimes committed by the two members of a pair, and there were also striking resemblances in temperament, intelligence, and general behavior. In reading Lange's book one can hardly resist the impression that these twins are under the spell of a fatalistic urge to similar kinds of misconduct. That identical twins commit the same types of crimes may be attributed in part to their close association, but close association without identical heredity fails to produce the same results.

The studies of Legras on several twin pairs in Holland are confirmatory of the findings described in that in all the four pairs of monozygotic twins observed both members were criminal, whereas in each of the five pairs of dizygotic twins criminality was confined to one member of a pair. More recently Rosanoff, Handy, and Rosanoff have investigated a fairly large series of twins among adult prisoners, juvenile delinquents, and children presenting problems of behavior. The results are summarized in Table 4 .

It appears that when one member of a pair of monozygotic male twins has a prison record the other member was a convicted criminal also in twenty-two of the pairs, whereas only one member was convicted in eleven cases. Among the 
Table 4.-Criminality, Juvenile Delinguency, and Behavior Problems in Twins $^{1}$

\begin{tabular}{|c|c|c|c|}
\hline Type of twins & $\begin{array}{c}\text { Adult } \\
\text { criminals }\end{array}$ & $\begin{array}{c}\text { Juvenile } \\
\text { delinquents }\end{array}$ & $\begin{array}{l}\text { Behavior } \\
\text { problems }\end{array}$ \\
\hline \multicolumn{4}{|l|}{ Like sex, probably monozygotic: } \\
\hline Males, both affected........... & 22 & 25 & 21 \\
\hline Males, one affected............ & II & 2 & 4 \\
\hline Females, both affected. . & 3 & 14 & 20 \\
\hline Females, one affected......... & I & I & 2 \\
\hline \multicolumn{4}{|l|}{ Like sex, probably dizygotic: } \\
\hline Males, both affected..... & 3 & II & I3 \\
\hline Males, one affected....... & 20 & 5 & 10 \\
\hline Females, both affected... & 2 & 9 & I3 \\
\hline Females, one affected............ & 3 & $\circ$ & 24 \\
\hline \multicolumn{4}{|l|}{ Opposite sex, dizygotic: } \\
\hline Both affected. . & I & 8 & 8 \\
\hline Only male affected.............. & 21 & 28 & 18 \\
\hline Only female affected... & $10^{\circ}$ & 4 & 3 \\
\hline Totals.......... & 97 & 107 & 136 \\
\hline
\end{tabular}

${ }_{1}$ After Rosanoff, Handy, and Rosanoff.

dizygotic twins there were only three concordant cases as compared with twenty which were discordant. In the juvenile delinquents both members of a twin pair were apt to have a bad record whether they were monozygotic or dizygotic. There was a much higher percentage of delinquencies among the females in this group, but most of these were due to sexual irregularities, which are apparently a very contagious type of delinquency in both types of twins. In general, the findings of Rosanoff, Handy, and Rosanoff are similar to those of Lange and Legras. The evidence which has now accumulated shows quite clearly that like heredity in environments which are as a rule of much the same kind is associated to a rather surprising degree with similarities in criminal careers.

\section{Suggested Readings}

Guyer ('27), Haynes ('30), chaps. 2, 4. Huntington and Whitney ('27), chap. 8. Lange ('30). 


\section{I82 HUMAN GENETICS AND ITS SOCIAL IMPORT}

\section{Questions}

I. What hereditary traits might dispose people to commit crimes?

2. Do you know any families in which bad temper or disagreeable dispositions seem to be inherited?

3. How do you account for the fact that in some primitive peoples stealing and lying are very rare, whereas other peoples are notorious for their dishonesty?

4. Are different peoples characterized by different degrees of excitability, impulsiveness, and revengefulness, and have these traits any connection with proneness to commit crime?

5. Does crime tend to be associated with insanity?

6. What do you think that, on the average, broken homes may imply as to the quality of the parents?

7. What reasons can you assign for the high percentage of crime in the United States?

8. How has crime in the United States been affected by immigration?

9. What can you find out about so-called crime areas in cities?

10. Does the similarity of criminal records in identical twins prove that propensities for particular types of crime are inherited, or does it simply indicate that similar environments may produce much the same kind of effects on persons of the same heredity?

Ix. Is the sterilization of criminals a justifiable procedure on biological grounds? on the ground that it would promote social welfare? 


\section{CHAPTER XIV \\ THE SOCIAL-PROBLEM PEOPLE}

7 HE social damage wrought by defective and disordered 1 mentality has been brought out very clearly by several studies of families showing a high percentage of feeble-mindedness and mental abnormality. One of the first of these studies was carried out by Mr. Dugdale, who in the course of an investigation of the prisons of New York found in one locality that a considerable number of the inmates were related by blood. This circumstance led Mr. Dugdale to ferret out the genealogical history of these prisoners, and he traced the stock back to a Dutch settler, Max Juke, who was born in the early part of the eighteenth century. The two sons of Max married into a family containing six sisters, five of whom were disreputable characters. Dugdale followed their descendants through five generations and unearthed a most unsavory record of pauperism, prostitution, illegitimacy, and crime. Among these descendants there were 50 prostitutes, 60 thieves, 160 paupers or recipients of poor relief, and 7 murderers. About one-fourth of the children were illegitimate. The cost to the state occasioned by the shortcomings of this group was estimated as $\$ 1,308,000$, but the indirect damage, moral and other, cannot be adequately measured. The descendants of some of the sisters were noted for pauperism; those of Ada Juke, known as Margaret, the mother of criminals, were especially notorious for crime.

Such was the melancholy record of this family at the time Dugdale wrote its history in 1875 . About forty years later, Dr. A. H. Estabrook investigated the further history of the 


\section{I84 HUMAN GENETICS AND ITS SOCIAL IMPORT}

family, and found that by I9I 5 it had increased to I, $25^{8}$ living members, but that there was little improvement in the character of the later generations. Taking the whole family up to I9I5, 378 were prostitutes, 86 keepers of brothels, and i 8 were criminals. Of the 698 children who had attended school, 394 were retarded three or more years, and 107 were mentally deficient to such a degree that they needed institutional care. According to Estabrook probably 40 per cent of the Jukes were mental defectives.

The history of the Kallikak family, which is the subject of a fascinating chronicle by $\mathrm{H}$. H. Goddard, is in many respects parallel to that of the Jukes. One branch of this family took its origin in an affair between a revolutionary soldier, Martin Kallikak, and a feeble-minded girl whom he met in a tavern. As a result this girl had an illegitimate son whom she named Martin Kallikak, Jr. Young Martin married and raised a family of ten children. These proved to be idle and shiftless individuals given to vice and petty crime. Of the 480 descendants of this branch of the family, the investigators were able to secure records of the mentality of only I 89 persons, but of these I 43 were adjudged to be mentally deficient and 43 were considered normal. In this group, according to Goddard, "There have been 36 illegitimate children, 33 who were sexually immoral, mostly prostitutes, 24 confirmed alcoholics, 3 epileptics, 82 died in infancy, 3 criminals, 8 kept houses of ill fame." There were $\mathrm{I}_{1} \mathrm{4} 6$ individuals resulting from marriages of the Kallikaks with other families, usually of a low grade of mentality. Of these 262 were rated as feeble-minded, I97 were considered normal, while the mental status of $58 \mathrm{I}$ was undetermined.

Besides the strain of degenerate progeny, there are other descendants of Martin Kallikak, who make quite a different showing. After the episode with the feeble-minded girl, 
Martin married a Quaker woman of good ancestry. Seven children resulted from this union. All of these children married into good families and their descendants included lawyers, doctors, judges, educators, and prominent citizens of many kinds. There were no feeble-minded, or illegitimate children, and little alcoholism or immorality.

These two sharply contrasted strains doubtless owe their characteristics to a combination of hereditary and environmental causes. Even though it may not have been possible to make very accurate estimates of the mentality of many individuals in such stocks, there is little doubt that both the Jukes and the Kallikaks were characterized by a high percentage of mental deficiency. Some individuals have been rated by trained observers; others were classified on the basis of less trustworthy evidence. In most cases one would not go far wrong in assuming that the village idiot was mentally deficient even in the absence of any scientific tests. With borderline individuals and persons who have had poor educational advantages rating' on the basis of hearsay evidence may be an unsafe procedure. Granting that an injustice may have been done to some worthy individuals of the Juke and Kallikak families, enough is known about many of them to indicate quite clearly their mental inferiority. Some individuals have become respectable and fairly intelligent members of society, especially those who migrated away from the rest of the group. Both stocks represent a great variety of different heritages and great inequalities of mental endowment are to be expected. Were it not for their bad environment, both groups would probably have been less objectionable.

In considering the roles of environment and heredity in such stocks as the Jukes and Kallikaks, we should bear in mind that bad heredity has a strong tendency to create bad environment. Low mentality tends to go along with 
poor education and an inferior economic and social status. Pauperism, vagabondage, illegitimacy, and intemperance tend sooner or later to become a part of the traditional mores of the group. People of this class are prone to mate with their own kind, and as a result whole communities grow up characterized by a large amount of consanguinity which brings out undesirable recessive traits. This fact is illustrated in the history of several notorious stocks such as the Tribe of Ishmael, the Zero family, the Nams, the Hill Folk, the Pineys and other human derelicts. Some strains, due probably to tradition, are characterized by criminality, others by pauperism or vagabondage, and others again by prostitution or intemperance, but most of these stocks have more than their quota of all these undesirable characteristics. Recently Lidbetter has published a detailed study of the paupers of East London, in which he shows that there are pauper stocks whose members subsist mainly upon charity, generation after-generation. A number of these lines have a high percentage of insanity, epilepsy, and mental and physical defectiveness.

Stocks of the kind described do not include very many defectives of the lowest grade. They consist of persons who are naturally dull or stupid together with a number with normal intelligence. Many of them can be improved greatly by training and good surroundings, although there are others who are hopeless under any circumstances. Such stocks are sometimes referred to as constituting the "socialproblem group." Social problems are not confined to any single stratum, but the stratum lying above the level of imbecility and below the average level of intelligence, furnishes much more than its quota of problems of many sorts. Where there is mental abnormality as well as defective intelligence, which is not infrequent, the social damage is considerably greater. 


\section{THE SOCIAL-PROBLEM PEOPLE}

\section{Suggested Readings}

Dugdale ('O2), Goddard ('12), Lidbetter ('34). See also Estabrook ('16) and ('23), and Estabrook and Davenport ('12), Winship (1900).

\section{Questions}

I. What are the reasons for the high birth rate in such stocks as the Jukes and Kallikaks?

2. What influences tend to offset the high birth rates of these stocks?

3. Is it better for such stocks to marry their own kind or to marry into the normal population?

4. Do you think that better environment and educational opportunities would lead to an increase or to a decrease of the social-problem group?

5. What influences tend to bring people of the social-problem group into the same localities? 


\section{CHAPTER XV}

\section{CHOICE IN MATING}

TN SEXUALLY reproduced organisms the kinds of $\mathcal{I}$ individuals that are produced depend upon the kind of matings which take place among the progenitors. Among the higher animals reproduction is not entirely determined by chance associations, but each sex may exercise a certain amount of choice in the selection of mates. If the animals which are chosen as mates differ on the average from those which are rejected, selective mating cannot fail to have at least some influence upon the course of biological evolution. Choice in mating affords an opportunity for psychological factors to modify the hereditary qualities of a species. According to Darwin, choice in mating, or sexual selection, is an important subsidiary factor in the evolution of the higher animals. A large part of Darwin's Descent of Man is devoted to presenting evidence for the operation of sexual selection in causing the evolution of so-called secondary sexual characters. Darwin attempted to explain the more striking coloration of male birds, their powers of song, and their instinct to display their attractions during courtship, as due to a continued selection by the females of the more aesthetically pleasing males. Not only in birds, but to a certain extent in mammals, reptiles, and even amphibians, fishes, insects, and spiders, the males are distinguished by characters which would appear to be more ornamental than useful. Since these features could hardly have been evolved by natural selection, Darwin conjectured that they owe their existence to sexual selection. They were deemed useful 
at least in the sense of enabling their possessors to succeed in mating and perpetuating their kind.

This ingenuous and plausible theory aroused much adverse criticism into the merits or demerits of which we shall not enter. Whether or not the beard, deeper voice, and other secondary sexual characters of the human male owe their origin to sexual selection, as Darwin maintained, there is no doubt that this factor has played a not unimportant part in human evolution and that it is operative in full vigor at the present time. People obviously do exercise choice in mating, at least in modern civilized society, and the way in which this choice is exercised has an important influence in determining the quality of the children who are born. The deformed, the sickly, the repulsively ugly, and those with mean and surly dispositions are fortunately less successful in the choice of mates than the healthy, comely, physically vigorous, and kindly disposed. From the racial standpoint this is all to the good. Dr. Knight Dunlap in his little volume on Personal Beauty and Race Betterment contends that beauty of face and figure are the outward and visible signs of racially valuable qualities of mind and body. We are pleased with characteristics indicative of vitality, intelligence, quick sympathy, and geniality. The secondary sexual characters suggestive of reproductive capacity have an appeal to the mating instinct as has been stressed by Mr. Havelock Ellis. In so far as sexual selection is determined by the native impulses of human beings, it is doubtless eugenic in its effects. The attraction of women for strong, manly, and intelligent men, and the tendency of men to choose beautiful, vivacious, and kindly women, are forces ever working to produce a superior race of human beings. Although in primitive society the free exercise of choice in mating was much restricted by rigid tribal customs, taboos, and the exercise of parental authority, women, 


\section{I9० HUMAN GENETICS AND ITS SOCIAL IMPORT}

as Howard, Westermarck, and others have shown, nevertheless enjoyed a certain amount of freedom of choice in marriage. Greater liberty of choice was exercised by the men; and the custom in many tribes of not allowing a man to marry until he had demonstrated his power and courage by some sort of feat at least kept the weaklings from propagating their defects. On the whole, sexual selection in primitive society was probably a force working for race betterment.

The development of modern civilization, and especially the great changes that have occurred in industry, have had their effects upon sexual selection as they have upon other factors of biological evolution in the human species. The natural predilections of men and women for superior individuals of the opposite sex undoubtedly continue to influence marriage selection, and will always do so as long as human nature remains the same. But other forces have entered upon the scene which tend to put sexual selection on a very different basis.

Properly to evaluate the workings of selective mating we should have some means of comparing those who are mated with those who are not. A study of this kind has never been undertaken for any community of human beings. We cannot assert positively whether in any city or country those who are married are either superior or inferior to those who are not. Certainly common observation discloses many excellent and attractive people who are unmarried, and also many married people whose repulsive qualities make one wonder how they succeeded in attaching themselves to their unfortunate mates. In endeavoring to ascertain how sexual selection works out we have to base our conclusions on the marriage rates and ages at marriage of different classes of the community. Concerning the proportion of men in different occupational classes who are 
married in the United States the following data have been compiled by the Census of Igoo.

Table 5.-Percentage of Men Married in Different Occupational Groups in the United States

\begin{tabular}{|c|c|c|}
\hline Groups & $\begin{array}{l}\text { Percentage married } \\
\text { over } 15 \text { years of age }\end{array}$ & $\begin{array}{l}\text { Percentage married } \\
\text { over } 25 \text { years of age }\end{array}$ \\
\hline 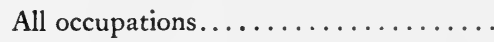 & 71.4 & $59 \cdot 5$ \\
\hline 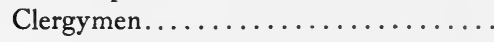 & 96.8 & $79 \cdot 5$ \\
\hline Physicians and surgeons............. & 95.0 & $77 \cdot 7$ \\
\hline 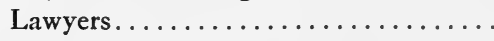 & $93 \cdot 3$ & 72.0 \\
\hline Farmers except laborers............. & 9 I. 6 & $87 \cdot 3$ \\
\hline 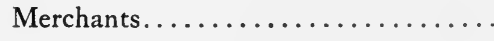 & 90.9 & $79 \cdot 3$ \\
\hline 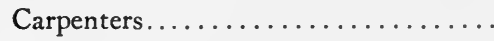 & 89.8 & $77 \cdot 5$ \\
\hline Teachers............. & $69 \cdot 4$ & $47 \cdot 2$ \\
\hline Salesmen............. & $59 \cdot 4$ & 43.2 \\
\hline Farm laborers.......... & 30.3 & 19.2 \\
\hline
\end{tabular}

The percentage married in these occupational groups depends to a considerable degree upon age as is indicated by the percentages aged twenty-five or over. The low percentage married among farm laborers in I 900 is largely due to the fact that as a group they are young, only 30.3 per cent being twenty-five or more years of age. The percentage of men in the professional groups who marry is fairly high. Cattell has found that the percentage of American men of science who were married was 89.5 , and Huntington and Whitney found that the per cent of men who have married in Who's Who in America (1926-1927), was 87.3, or probably somewhat higher if reasonable allowance is made for those who failed to state whether they were married or not.

The percentage of male college graduates who marry is about the same as in the general population, although the age at marriage is considerably higher. We have already pointed out that the percentage of women graduates who marry is low, being on the average between 50 and 70 per cent. In many occupations especially of a higher grade the 


\section{I92 HUMAN GENETICS AND ITS SOCIAL IMPORT}

percentage of women who remain unmarried is high. It is especially high among teachers. The proportions of women who are gainfully employed have been increasing for several decades. As a result, a large proportion of the more capable women remain single to the detriment of our racial heredity.

The age at marriage in both men and women is higher among the educated than in the uneducated classes. Among graduates of both sexes and still more among the members of the learned professions, it is considerably higher than in the general population. Skilled laborers marry later than the unskilled. Those engaged in agricultural pursuits marry earlier than city dwellers. Notestein has compiled data on the ages of wives at their first marriage in the United States from I900-I905, and the percentages married are given in the following table:

Table 6.-Percentages of Women at Time of First Marriage in Different Age Groups in Sample Area of the United States, 1900-I905. ${ }^{1}$

\begin{tabular}{c|r|r|r|r|r}
\hline \multirow{2}{*}{$\begin{array}{c}\text { Age at first } \\
\text { marriage }\end{array}$} & \multicolumn{5}{|c}{ Occupational group } \\
\cline { 2 - 6 } & Professional & Skilled & Unskilled & Farm owner & Farm laborer \\
\cline { 2 - 5 } 15-19 & 8.2 & 31.5 & 39.9 & 28.7 & 51.3 \\
$20-24$ & 42.5 & 46.2 & 37.8 & 44.5 & 36.3 \\
$25^{-29}$ & 36.2 & I 5.7 & 14.4 & 17.7 & 9.5 \\
$30-34$ & 9.5 & 4.8 & 4.5 & 6.0 & 2.2 \\
$35^{-44}$ & 3.5 & 1.8 & 3.4 & 3.1 & 0.7 \\
\hline
\end{tabular}

1 Data from Notestein.

The age at marriage for wives has a greater effect upon fertility than that of the husband, although the age of the latter has also a considerable influence. Ages of husband and wife at the time of marriage show a certain degree of correlation in that both tend to be high in the higher occupational groups. It may be noted that the relation of age at marriage to occupational status is much the same as the relation of fertility to occupational status, and doubtless the latter 
relation is in part determined by the former. Contrary to a common opinion there is a strong tendency for like to mate with like. Data collected by Galton showed that people of similar eye color tend to mate more frequently than people of dissimilar eye color. This conclusion was confirmed by the more extensive studies of Pearson who showed also that there is a tendency to assortative mating on the basis of stature. The tendency of the tuberculous to marry the tuberculous, and the deaf to marry the deaf, may be due to the segregation of such people in a common environment, and perhaps in part to a mutual sympathy arising out of their common affliction. There is a considerable degree of assortative mating on the basis of education and social status. As a rule educated men marry educated women and ignorant men marry ignorant women. Mental defectives tend to mate with mental defectives as is abundantly illustrated in the chronicles of the Jukes, Kallikaks, Tribe of Ishmael, and other notorious degenerates.

Propinquity is a potent influence in determining the choice of a life partner and accounts for many alliances otherwise inexplicable. D. M. Marvin found that in 49,000 marriages in Philadelphia people married others of the same occupation twice to three times as frequently as they would if matings were due to chance. People of similar kind are of ten thrown together as a result of their occupations or their social positions. In inbred communities persons of common descent are brought into association thus causing a certain amount of assortative mating. The same tendency is of ten fostered by religion. There is more or less social pressure leading people to marry others of the same religious belief. This acts quite strongly in preventing marriages between Protestants and Catholics and between Christians and Jews. Within the various Protestant sects the tendency to marry within the fold is due much more to association than 


\section{I94 HUMAN GENETICS AND ITS SOCIAL IMPORT}

to differences over doctrine. Formerly the Quakers and other sects expelled members who married outside the group, but such regulations are now largely abandoned, and the whole influence of religion in breaking up a population into isolated groups is much relaxed.

In general, people prefer to mate with others of the same race or natural group. This is partly a result of the natural clannishness of the human species and partly due to other factors, regional and social. Most of the marriages between immigrants in the United States occur among people who come from the same country. In the second and subsequent generations of our own immigrant population there are more marriages between members of different national groups. Our census data on the proportions of our population of mixed parentage are apt to give a misleading impression of the extent of intermarriage between persons of different racial or national origin. An Italian who marries a nativeborn American is apt to choose one of Italian parentage. In a study of marriages between native born and foreign born parents of students in the University of California I have found that such marriages are largely confined to persons of the same general stock. Thus in marriages between an Englishman and a native-born woman, the wife was of English parentage in 42 cases, American in I9, Scotch in I I, German in II, Irish in 10, French in 8, Scotch-Irish in 4, with a smaller number of cases of other European nationalities. German men married native-born women of German parentage in $5^{8}$ cases, American in 18 , French in 4 , and in no more than two instances did they marry members of any other one national group. And it is likely that a fair proportion of the wives classed as native American were of the same derivation as their husbands.

The so-called "melting pot" is not blending our immigrant stocks as rapidly as is often supposed. These stocks fre- 
quently persist as inbreeding groups for many generations. The out-marriages which occur are most apt to be between related peoples such as the English, Scotch, and Germans, rather than between these groups and the Italians, Greeks, or Armenians. Nevertheless, the process of racial assimilation is slowly going on and is contributing to enhance the heterozygosity of the native Americans.

\section{Suggested Readings}

Darwin, C. ('7I), part III. Darwin, L. ('26), Dunlap, ('20). Holmes ('2I ), chap. Io ('23), chaps. 9, Io. Popenoe and Johnson ('33), chaps. II and I4.

\section{Questions}

I. Look up Darwin's treatment of sexual selection in the human species in The Descent of Man. What characteristics of the male sex are supposed to owe their origin to sexual selection?

2. Contrast the working of the "law of battle" with the effects of female choice.

3. In what respects is sexual selection working eugenically and in what respects is it working dysgenically under present conditions?

4. If you wished to ascertain the general effects of sexual selection in man, what kinds of facts would you like to have collected?

5. What can you say of frequency of marriage among celebrated women as compared with celebrated men?

6. In what respect would you think that late marriages would have a eugenic effect?

7. What laws in your state affect choice in marriage? Which of them are desirable and which are the reverse?

8. In what ways might sexual selection be made a potent factor in racial improvement? 


\section{CHAPTER XVI}

\section{THE BIRTH RATE AND THE CAUSES OF ITS DECLINE}

U $\mathrm{P}$ TO this point our discussion has been devoted to the principles of heredity and the varied types of human heredity, both physical and mental. Hereditary variability forms the basis of all biological evolution. And since the human species is characterized by a very unusual amount of genetic diversity, it is capable of relatively rapid evolutionary changes.

The direction which evolution takes depends upon (I) what kinds of variations appear, and (2) what variations are able to increase most rapidly in numbers. That human evolution may take many directions is shown not only by our great intraspecific variability, but by the fact that mankind has become diversified in so many ways in different parts of the globe. Consider the varied physical characteristics of Hottentots, hairy Ainus, Indians, Nordics, Polynesians, Mongolians, and African pigmies, and it will be apparent that as the human species has spread over the surface of the globe it has diverged along numerous branching and rebranching lines. The kinds of variations which come to prevail at any time or place depend upon their relative rates of natural increase, and this depends upon relative fertility and relative mortality. If two stocks have the same degree of fertility, the one having the lower death rate will eventually prevail. If both have the same mortality, the one with the higher fertility will prevail. What counts in the process of evolution is, of course, the balance of 
births and deaths. Both factors are always acting in the drama of life, but it will be convenient to treat of them separately.

In studying birth rates, it is desirable to have some numerical measure of their magnitude. The measure most commonly employed is the so-called crude birth rate, or the number of births a year per thousand population in that year. It does not give a very accurate measure of the actual fertility of a people because it is affected by the proportions of the sexes and the age composition of the population. The more people in a country who are either too old or too young to have children, the lower the birth rate. Since the age composition of the population varies greatly in different countries, birth rates are sometimes expressed by the number of births per thousand women in the child-bearing period, which is commonly taken to be from fifteen to forty-five years of age. This rate is affected by the proportion of women who are married, and hence birth rates are sometimes estimated in terms of the number of births per thousand married women in the child-bearing period. For countries in which the percentage of illegitimate births is low, this measure of the birth rate affords a fairly satisfactory index of the relative fertility of the female population.

It is often desirable to distinguish between reproductive capacity and actual reproductive performance. The Population Association of America has recommended that the term fecundity be applied to the former, and fertility to the latter. We shall conform to this usage, although some writers employ these terms in the reverse sense. The fecundity (i.e., reproductive capacity) of the normal human female is quite high. Records of over twenty births are not uncommon among the women of certain peoples, although even in primitive society the physical limit of fecundity is seldom reached. The liability of a woman to conceive falls off rather 


\section{I98 HUMAN GENETICS AND ITS SOCIAL IMPORT}

rapidly with increase of age at marriage. Galton has estimated the fertility of women marrying at ages seventeen, twenty-two, twenty-seven, and thirty-two as roughly in the ratio of six, five, four, and three respectively. An increase of the average age at marriage, therefore, would have a potent effect in lowering the birth rate.

The birth rates of the races and peoples of the globe show very great inequalities. During the past century the birth

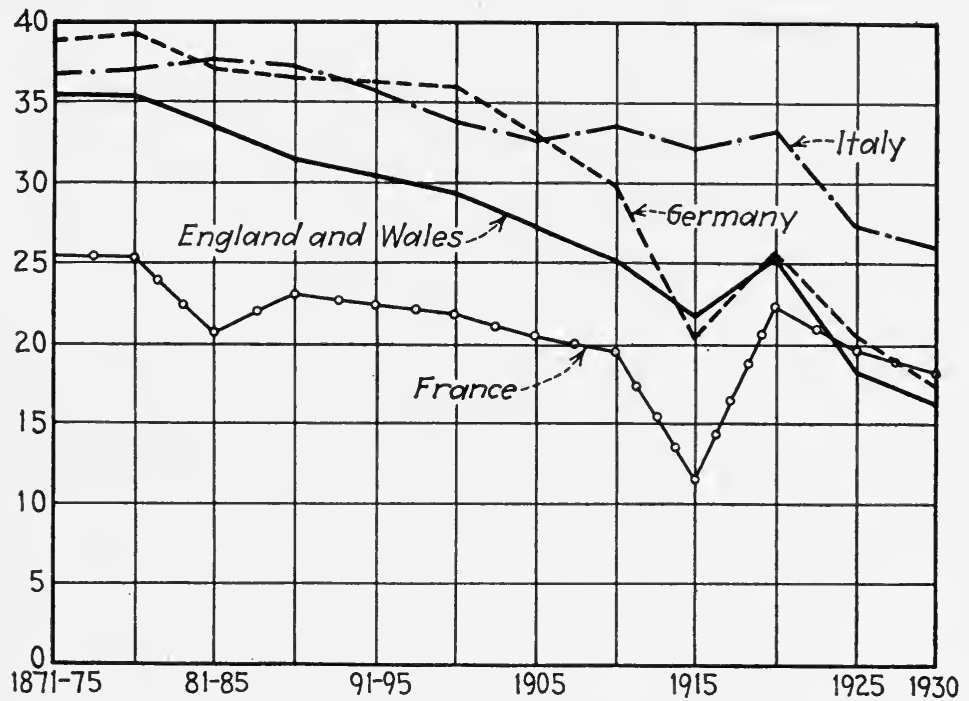

Fig. 60.-The decline of the birth rate in England and Wales, France, Germany, and Italy, I 87 I to I930.

rate among nations of advanced culture has undergone a marked decline. There is more or less uncertainty as to its trend in most countries before 1850 . In the Scandinavian countries and in France the birth rate seems to have slowly declined from near the beginning of the nineteenth century, with more or less irregular fluctuations due to wars and conditions of trade. Until recently the birth statistics of the United States have been very inadequate, but to judge from the decreasing proportion of children under five years of 
age given by the reports of the Census, the birth rate has been declining since about I810. In England, Germany, and some other European countries the decline of the birth rate set in during the seventies, while in southern and eastern Europe it did not begin to decline until later and still continues to be fairly high. In Australia and New Zealand the birth rate, which was quite high in the seventies and eighties, has rapidly fallen until it is now little more than

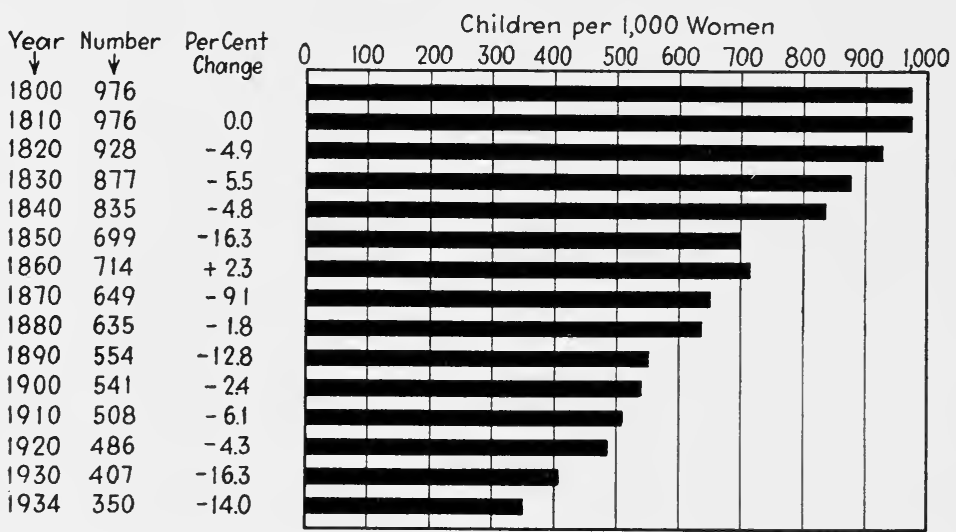

FiG. 61.-Number of children under five years of age per 1,000 women sixteen to forty-four years of age inclusive in the United States from 1800 to 1934 .

half as high as it was fifty years ago. Among the Asiatic peoples the birth rate is high, although in most countries accurate birth statistics are not available. In Japan the birth rate has been over 30 per thousand (32.4 in 1930) ever since reliable birth records have been kept.

Where birth rates are high, death rates are usually high also, although these rates vary independently to a considerable degree. As the birth rate has fallen, the death rate has fallen, and at times the more rapidly, so that the rate of natural increase has become greater. This was a not uncommon condition in European countries during the latter half of the nineteenth century. But for several years past, the 
excess of births over deaths has in general been growing less. There are two reasons for this. First, there is a natural limit to the reduction of the death rate, and, as this limit is approached, further reductions are made more slowly. Second, in the period following the World War the birth rate in Europe and in the United States has fallen to unprecedented levels. Every year sees the populations of the more advanced countries drawing more closely toward a stationary condition. And the present financial depression has apparently accelerated the decline.

In seeking for the causes of the decline of the birth rate, we should distinguish between a purely statistical decline, and one which is caused by a reduction in the average number of children to which each woman gives birth. When we measure birth rates by the number of births per thousand inhabitants it is evident that if the mortality of nonreproducing individuals is decreased while the number of births remains the same, the birth rate will go down. A reduction of infant mortality produces a population with relatively more children, and hence depresses the birth rate. A good illustration of a largely spurious decline of the birth rate is afforded by the Japanese in California. Soon after the so-called picture brides entered the state, the Japanese population consisted largely of recently married adults with few children. The number of children born per thousand Japanese in the state soon came to be very high. As more children were born the Japanese population consisted of relatively fewer reproducing individuals and hence the birth rate went down. It would be bound to fall, even though each Japanese woman continued to bear children at the same rate. If we wished to compare the fertility of Japanese women with that of other women in the population it would be better to consider the number of births per thousand married women of child-bearing age. 
It is evident that one factor in the decline of the birth rate as commonly expressed is the reduction of the death rate, and especially infant mortality, which has occurred during recent decades. Rates are affected by causes which influence the denominator as well as the numerator of the fractions by which they are expressed. The immigration of persons in the child-bearing period of life would tend to increase the birth rate and decrease the death rate. Birth and death rates of individual towns and cities, for instance, are influenced considerably at times by the presence of colleges, hospitals, prisons, and other institutions which bring in people whose fertility or mortality rates differ from those of the general population.

The causes which affect real fertility are many. Among these are the factors which determine the frequency of marriage and age at marriage. Both age and rate of marriage vary considerably from country to country, and they have their evident effects upon fertility. As may be seen in the table, the proportion of women who are married in the younger age groups varies greatly in different countries. In Sweden, Germany, and England and Wales less than 2 per cent of the women between ages fifteen and nineteen are married, while in Bulgaria over Io per cent are married. In Bulgaria 63.3 per cent in ages twenty to twenty-four are married, while in England, Germany, and Sweden less than 30 per cent are married. As is shown by the data on the fertility of married women at different ages the birth rate of women rapidly declines as they grow older. Hence where a large proportion of the younger women are married the birth rate tends to be high.

While age and frequency of marriage may be important factors in causing the differences between the birth rates of different countries, they have been rather minor factors in causing the decline of the birth rate within each country. 
The reason for this is that these factors have either changed but slightly, or else such changes as have occurred would tend to increase the birth rate instead of causing it to decline. During several decades the proportion of women who are married in the United States has been increasing. This is especially noticeable in the younger age groups. In 1890 the percentage of men between twenty and twenty-four

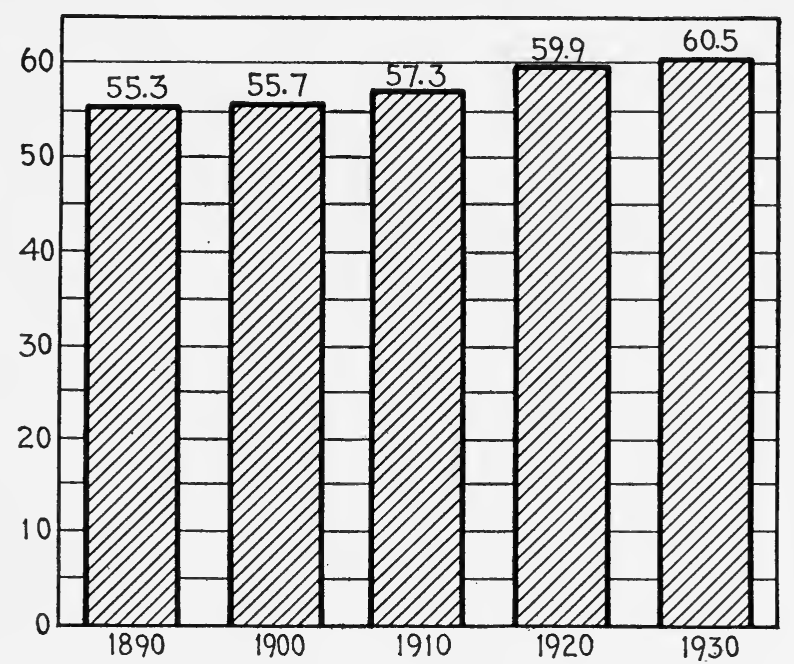

FIG. 62.-Percentages of the population of the United States who were married, 1890 to I930.

who were married was 22.2 ; in 1930 it was 30.9 . In 1890 the percentage of women between twenty and twenty-four who were married was 50.3 ; in 1930 it was 54.4 . In most countries of Europe the average age of both men and women at marriage has been declining slowly for several decades. The percentage of women in Europe who are married has fallen in some countries and risen in others, but, on the whole, changes in marriage rates have had no marked influence on the fall of the birth rate.

Some writers have ascribed the decline of the birth rate largely to hereditary changes in fertility. Herbert Spencer sees in the decline of the birth rate an illustration of a general 
biological law based on "the inverse variation between individuation and genesis." As more energy is expended in intellectual tasks, less is available for the perpetuation of the species. Education, he points out, leads to sterility, which is most pronounced in women, but is manifested to a less extent in men. Consequently with advancing civilization the birth rate tends to decline.

Professor Gini thinks that human races pass through cycles of fertility. Primitive races are fertile, but as civilization advances they undergo degenerative changes which involve diminished fertility and finally extinction. As stocks rise and go to seed they are replaced by more primitive peoples who have retained their pristine vigor and fertility. Here an appeal is made to hypothetical biological forces, which for some reason cause cyclical changes in fecundity. Whether such an appeal is necessary depends largely on whether or not other factors exist which might account for the phenomenon.

Dr. G. R. Wagner-Manslau thinks that the "declining birth rate is a huge selection of modern civilized peoples toward weak desire for the child brought about by marriage for money." Its basis is conceived to be genetic. Since people with few children are more apt to rise in the social scale, and since there is a tendency to marry the more successful types, there is thus brought about a general decline of the birth rate. But even if a social selection may have led to a decline in the fecundity of the upper social strata, it can hardly have led to a decline in the proletariat or in the general population.

Naturally, the birth rate is influenced by factors affecting the general health and vigor of a people. Women living in a rural environment where they engage in manifold labors are affected by natural sterility much less than their urban sisters, who live under conditions much more unfavorable 


\section{HUMAN GENETICS AND ITS SOCIAL IMPORT}

for the development of physical vigor. Many women who want children are unable to have them owing to physiological causes that are often obscure, but which operate with greater force in women living in an urban environment. This may be partly due to the nervous tension under which so many women live. More of it probably results from the lack of wholesome physical exercise in the open air, or from irregular unhygienic modes of living.

Among the causes which affect fertility, venereal diseases unquestionably occupy an important place. Gonococcus infection is a not uncommon (perhaps the most common) cause of sterility in the male sex because of the blocking of the vasa deferentia which prevents the exit of spermatozoa. Sterile marriages are frequently due to the absence of spermatozoa in the seminal fluid. According to Meaker the male is responsible for about a third of the cases of sterility in marriage.

In women gonorrhea of ten causes severe havoc. In many cases a woman contracts the disease from her husband after he thinks he is cured. The infection may extend to the uterus and Fallopian tubes, and even the peritoneum. If a pregnant woman is infected, the baby may contract the disease during delivery and become blind. After delivery the infection may spread rapidly and lead to inflammatory conditions which later result in sterility. Many one-child marriages result from the devastations of this disease. The percentage of cases of sterility due to gonorrhea have been variously estimated as from I 3 to 50 per cent. Often the disease lingers on in a latent form in which it may escape notice. According to Alexander four-fifths of all women who are infected are unaware that they have this disease.

Syphilis causes sterility less frequently than gonorrhea, but since it is a prevalent cause of stillbirths and affects the birth rate indirectly by undermining vitality it is an 
important factor in reducing fertility. The proportion of stillbirths due to syphilis has been estimated by Dr. Willey as 32.8 per cent. The damage done to health by the two chief venereal diseases is incalculable. How widespread these diseases are is difficult to ascertain. Their prevalence varies greatly in different countries and cities. According to reports from eight hundred physicians of Hamburg, Germany, I. 3 per cent of the men between fifteen and fifty contract syphilis each year, and Lenz estimates that 45.5 per cent of infection is a minimal percentage, while Weinberg estimates the percentage of syphilitic males as 50. These estimates based on urban populations have been considered by some physicians as much too high. The examination of the recruits for the U.S. Army revealed about 5 per cent of syphilitic infection, but only the more obvious cases were detected. Gonococcus infection is more common. According to Lenz, from 40 to 50 per cent of all men and from 20 to 25 per cent of all women in Germany have contracted the disease at least once. Knowledge of prophylactic measures whose partial efficacy has been demonstrated in the U. S. Army and Navy has doubtless become much more widespread than formerly. Partly for this reason, syphilis has decreased, but this is due also to improved methods of treatment which shorten the infective stages of this malady. While venereal diseases decrease the birth rate they are probably not responsible for its downward course because they were widely prevalent long before the birth rate started to decline.

In the opinion of most students of the subject the greatest factor in the decline of the birth rate is the voluntary restriction of the birth supply. As Levasseur has stated in discussing the declining birth rate of France, "It is superfluous to look for subtle causes. The decisive fact is simple: the families in France have not many children because they 


\section{HUMAN GENETICS AND ITS SOCIAL IMPORT}

do not want to have many children." If the decreasing birth rate has been largely brought about voluntarily, there are two aspects of the subject to be considered: (I) the reasons why people desire fewer children, and (2) the means by which the desired end is achieved. Our ancestors took childbearing as a matter of course. People obeyed their reproductive impulses, and if God decreed that they have children they accepted the situation and got along to the best of their ability. For the Rev. T. R. Malthus the only feasible way of avoiding the burdens of large families is "prudential restraint." This involved the postponement of marriage until the reduction of fertility would decrease the population to a point at which all could enjoy a fair measure of prosperity. The idea that there was any way of checking the birth supply without having recourse to means that were positively criminal never entered the heads of most of the great grandmothers of the present generation. Aside from delayed marriages and prudential restraint within marriage the only methods of preventing large families known to most people were infanticide and abortion, and these were not resorted to except under very unusual conditions.

A change of attitude began to appear after the origin of the birth control movement. Contraception had been practiced to a certain extent from early times, but it was not employed extensively by modern civilized peoples before the nineteenth century. Especial importance has been attributed to the Bradlaugh-Besant trial in 1877 . This arose over the publication of a book by Dr. Knowlton on The Fruits of Philosophy in which contraceptive methods are described and advocated as a means of relieving mothers from the burdens of a large family. The issue of this work in England by Charles Bradlaugh and Mrs. Anne Besant led to the prosecution of the publishers, who received a heavy sentence, which was revoked by a higher court. Contracep- 
tion had been advocated previously by Francis Place, Richard Carlile, Robert Dale Owen, and others, but the rank and file of the population were little affected by their propaganda. The excitement created by the BradlaughBesant trial brought the subject prominently before the public mind and affords an excellent illustration of how movements are sometimes powerfully advanced by a little persecution. The immediate result was to increase enormously the circulation of Knowlton's book and subsequent pamphlets on the same subject, among which was Mrs. A. Besant's Law of Population, which ran through several editions and sold several hundred thousand copies. According to N. Himes, "No less than a million tracts furnishing contraceptive information were sold between 1876 and I89I."

About the time of this trial the birth rates in England began to decline. To how great an extent the trial was responsible for this is uncertain. Pearson is inclined to emphasize the influence of the passage of the Factory Acts, which limited the employment of children in industry. Child labor had come to constitute an important factor in the textile and other industries of England. Children were kept at work for long hours amid the most unhygienic conditions, sometimes chained to their tasks, "fed upon the cheapest and coarsest food; and slept by turns and in relays in filthy beds which were never cool." Mortality was appallingly high, but the supply was plentiful and cheap. The outcry against these barbarities led to the passage of a series of acts to regulate the ages and hours at which children could be employed. The Education Act of 1876 making school attendance compulsory also contributed to mitigate the evil. The employment of children in the textile industries was curtailed in England in 1878, and it is noteworthy, as Pearson has pointed out, that in manufacturing cities, such as Bradford and Leeds, there was a marked fall in the 
birth rate at about this time. The child was no longer a financial asset and became a liability instead; so children like other commodities proved to be responsive to the law of supply and demand.

In the agricultural counties the birth rate did not begin to decline until several years later. Doubtless birth-control propaganda was not so widespread in the rural as in the industrial regions, but there was less temptation to limit the family. In the voluntary restriction of births there have to be both the will and the way. With the increase of population, the growth of cities, the wider dissemination of education, and a greater desire for higher standards of living, the custom of family limitation gradually became more prevalent.

It is customary to attribute the voluntary reduction of births largely to economic causes. James Bertillon brought together the statistics of several large cities including Paris, Vienna, and Berlin, showing that the poorer the district the larger the average size of the family. He concluded that "L'aisance en traine la stérilité." In support of this conclusion there is cited the fact that as prosperity has increased the birth rate has fallen. On the other hand, lack of adequate income is often assigned as the reason for family limitation. In cities where it is more inconvenient to have a large number of children, and where each additional mouth to feed imposes an added burden upon an already inadequate income, the economic incentives to keep the family small are very strong. Among the land owners in France it has become a settled custom to have only a few children in order not to subdivide the patrimony among too many descendants. It would seem, therefore, that we are justified in making two statements concerning the relation of economic factors to the birth rate; (I) as people become wealthy they tend to limit their families; and (2) people commonly 
limit their families for economic reasons. The existence of these apparently opposed tendencies has given rise to a great deal of confusion in discussions of the subject. The reconciliation of the apparent contradiction involved in these statements lies in the fact that wealthy people do not limit their families because they are wealthy, but because wealth brings other interests and is usually associated with a higher level of culture. There are social duties and distractions which make a large number of children an undesirable encumbrance. Besides, large families in the upper strata are unfashionable, and the influence of class sentiment keeps the children few in number.

It should be borne in mind also that the correlation between low fertility and wealth is partly due to the fact that it is easier for a family to rise in the economic scale if there are few children to support. On the whole, however, it is not wealth, but the things that go along with wealth that lead to small families among the well-to-do. Among these factors a prominent place must be given to education. In a study of the sizes of families sending students to the University of California I find that as the education of parents increases, the size of the family goes down. The results are shown in the following table:

Table 7.-Relative Sizes of Families According to the Education of Parents

\begin{tabular}{|c|c|c|c|}
\hline \multirow{2}{*}{ Education of father } & \multicolumn{3}{|c|}{ Education of mother } \\
\hline & Grammar school & High school & College \\
\hline Grammar school... & $4 \cdot 17$ & $3 \cdot 55$ & $3 \cdot 35$ \\
\hline High school....... & 3.89 & $3 \cdot 3^{8}$ & 3.14 \\
\hline College....... & $3 \cdot 44$ & 3.24 & 3.10 \\
\hline
\end{tabular}

The average number of children is highest where both parents have no more than a common school education, and lowest when both parents have attended college. 


\section{I0 HUMAN GENETICS AND ITS SOCIAL IMPORT}

In a more homogeneous population consisting of rural families of Connecticut, Whetten found that the average number of children decreased as the education of the parents increased, but that if only the native-born Protestant families are considered, the differences in family size almost entirely disappeared. Within this group, formal schooling apparently affected the birth rate very little.

As a rule, educated people are not content with a low standard of living, and they are quick to perceive that large families lower these standards. Naturally, knowledge of contraception would be more rapidly spread among them. To a certain extent a negative correlation between education and fertility is due to relatively late marriages, but it is demonstrable that this is a subordinate factor. A desire to succeed in life and to attain a higher social and economic status is more apt to be found among people of education. The object of their striving is not so easily attained if children are begotten early in life. Arsène Dumont has called attention to a process in human society which he calls "social capillarity." Individuals, as they rise from a lower to a higher class, like oil in a lamp wick, tend to limit the number of their offspring. Ambition thus leads to sterility. In a democratic society where a rigid caste system does not occur, this movement may go on freely. We have been doing much to give everyone the advantages of education in order that those with the capacity to profit by it most will be able to attain positions to which their natural ability entitles them. With education available for all, so that children of unusual merit from the poorest families will be enabled to forge ahead and qualify for success and sterility, the rapidity with which the differential birth rate operates will be greatly increased.

Another factor in the declining birth rate is the emancipation of women and their increasing employment in industry. 
Formerly the duties of the home required most of a woman's time. With numerous labor-saving devices and the fact that so many articles are made in factories that were formerly made in the home, the work of the housewife has been greatly reduced. Besides, many more occupations are made available for women. It is now no longer necessary to marry for support, and it is no longer a disgrace to be an old maid. A much larger proportion of the female sex is self-supporting, and many married women add to the family income by pursuing employments outside the home. Children inevitably constitute an obstacle to all these activities. Women have become more self-reliant and independent. In many countries they have acquired political rights equal to those of men, and equal property rights have gradually been accorded them. The life of continued childbearing and household drudgery that formerly fell to the lot of so many mothers of preceding generations has little appeal to the modern emancipated woman. More and more women are taking the regulation of the birth supply into their own hands.

How far religion influences the birth rate is difficult to estimate because its effects are so closely associated with those of other factors. Birth rates vary considerably with members of different denominations. The peoples of Catholic countries, Hungary, Spain, Portugal, and Italy, are as a rule more fertile than those of Protestant countries, such as England, Norway, Sweden, and Australia. In the United States families of Catholics are larger than those of Protestants. This is not due merely to the fact that Catholics are relatively more common among the families of recent immigrants from Southern Europe, but the same statement applies to older residents of the country. According to Huntington and Whitney the average size of the family among the parents of people in Who's Who was 5.3 for Mormons, 3.3 for Roman Catholics, 3.I for Baptists, 
2.7 for Congregationalists, and 2.6 for Jews. In studying the size of the families from which students of the University of California come, I find that it is 4.44 for Catholics, 3.48 for Protestants, and 3.73 for Jews. On the other hand, in Vienna, the population of which is mainly Catholic, the birth rate has fallen since the war to IO.O per thousand. In the parts of Germany which are predominantly Catholic the birth rate is higher than in those parts which are predominantly Protestant. The same statement is applicable to the cantons of Switzerland. The number of children per family in Prussia among Catholic, Protestant, and Jewish families in which the husband and wife were of the same religion were as follows:

Table 8.-Children per Family in Different Religious Denominations in Prussia

\begin{tabular}{|c|c|c|c|c|c|c|}
\hline Religion & $1880-1884$ & I 890-1 894 & $1900-1904$ & I913 & $1922-1924$ & 1926 \\
\hline Catholics. & 5.2 & 5.2 & $5 \cdot 3$ & $4 \cdot 7$ & 3.0 & $3 \cdot 3$ \\
\hline Protestants.... & $4 \cdot 5$ & 4.2 & 3.8 & 2.9 & 2.0 & 2.2 \\
\hline Jews......... & $4 \cdot 3$ & $3 \cdot 3$ & 2.8 & 2.2 & I .8 & \\
\hline
\end{tabular}

The Catholic Church has emphasized the obligation to obey the command "Be fruitful and multiply and replenish the earth." It has taken a decided stand against the employment of artificial methods of preventing conception. The Pope's encyclical letter states the position of the Church by saying. "Since the conjugal act is meant to create new life, those who willfully deprive it of its power, act contrary to nature and do something disgraceful and immoral." But notwithstanding the official pronouncements of the Church, the voluntary limitation of births is practiced extensively in Catholic countries. It was in France, whose population is mainly Catholic, that the decline of the birth rate first started and reached (until very recently) its lowest level. In most countries the average size of the family has 
been reduced among both Catholics and Protestants. When conditions render such limitation desirable, the Catholic Church does not object to family limitation on principle, although it has always emphasized the duty of procreating new life. It objects to accomplishing this end by unnatural means. In fact the Pope has explicitly stated that "social and eugenical indications should be considered if legal and moral means and certain limits are taken into account."

Probably the attitude of the Church has some effect in checking the limitation of the birth supply, but it is insufficient to counteract the social, educational, economic, and other incentives which lead people to limit their families. Some students of the subject consider that religion per se has very little effect upon the birth rate. In several Catholic countries cultural standards are relatively low, and this is often true of the Catholic population of certain areas as, for instance, in the United States. Associated with this is the lower economic status of the Catholic elements of the population. In the Catholic parts of Germany where the birth rate is high, the population is mainly rural as in Bavaria and East Prussia, or largely Polish. In the urbanized Protestant population of Saxony the birth rate is very low, while the Protestant city of Berlin is almost in the van of the low birth rate cities of the world ( 9.9 per thousand in 1930). Even Paris has a considerably higher birth rate (I4.7 in I930). It seems evident that where the educational, social, and economic status of a population favor a low birth rate, fertility is low regardless of the religion of the population.

In considering the causes of the declining birth rate one must not neglect the influence of fashion. The effect of fashion in this regard is not due to mere imitation. There come to be more or less settled customs in respect to reproduction due to various reasons. Family limitation is prac- 


\section{I4 HUMAN GENETICS AND ITS SOCIAL IMPORT}

ticed, not only from selfish motives, but because parents wish to give their children educational and other advantages which are only possible when the family is kept small in size. In countries with an abundant population parents are not apt to feel under a strong obligation to swell the numbers of the inhabitants. Why then should they continue to have children beyond the small number they can comfortably support? In France it has long been'customary to standardize the family size, and to a less extent the custom is coming to be followed in other countries. Large families are often looked down upon as an indication of parental folly. And most women do not care to incur the unfavorable comments of their neighbors for breeding like rabbits. The small family system is encouraged not only from motives of prudence, but because it is a part of good social form.

\section{Suggested Readings}

East ('23), chaps. 8-I I. Fisher ('32), Holmes ('32), ('33), chap. 4. Lorimer and Osborn ('34), chaps. 2-4. Pearson ('II). Thompson ('35), chaps. 9-I I.

\section{Questions}

I. What advantages and what disadvantages are there in the use of crude birth rates?

2. How is the birth rate affected statistically by an increase in infant mortality? Explain. How do you think that the actual number of births would be affected by this cause?

3. How would a decline of the birth rate affect our statistics on the prevalence of insanity, the mortality from cancer and from cholera infantum? Explain.

4. How and why would a decline of the birth rate affect the marriage rate?

5. How is the birth rate affected by a decline of the birth rate?

6. How would a reduction of mortality after age sixty affect the birth rate?

7. Why is the age composition of Germany more favorable for a high birth rate than that of France?

8. How is the crude birth rate of a city affected by the presence of a college, an old people's home, a maternity hospital? 


\section{BIR'TH RATE AND CAUSES OF ITS DECLINE 215}

9. What do you think of the relative importance of the following influences on the birth rate: religion, income, education, social position?

ro. Have the efforts of rulers to check the decline of the birth rate met with conspicuous success? What have been the chief reasons for endeavoring to increase the birth rate?

II. Suppose that the production of children were brought completely under voluntary control in all classes; do you think that the race would continue to reproduce itself without loss?

12. How did the low birth rate of France help her to pay her indemnity after the Franco-Prussian war?

13. What relations tend to prevail in different countries between birth rates, immigration and emigration? 


\section{CHAPTER XVII}

\section{THE DIFFERENTIAL BIRTH RATE}

$7 \mathrm{HE}$ fact that the decline of the birth rate is to a large

1 extent the result of volition helps to explain the ways in which it has worked out in different classes of the population. Formerly large families were found in all social classes. In a well-known study of the decline of the birth rate in London, David Heron found that there was a positive correlation between fertility and various undesirable social characteristics, such as mortality from tuberculosis, high infant mortality, insanity, and bad living conditions, but that this situation had developed during the preceding fifty years. In $185 \mathrm{I}$ the net fertility was in favor of the classes of the higher social status. In Germany, until within the nineteenth century, the well-to-do peasant married before the worker without property. Apprentices in trades seldom married, and persons belonging to guilds commonly deferred marriage until they attained a more or less assured position. Among the successful types there was little family limitation, and, according to Lenz, the effect of differential fertility was, on the whole, eugenic. According to Whetham, the average number of children to fertile marriages of the British landed gentry contracted between I8 30 and I $84^{\circ}$ was 7.I; but it fell to 3.13 from I88I-I890, a drop of over 50 per cent. Among the people whose names occur in Who's Who Whetham found that, excluding clerical and military families, there was an average of 5.2 children per marriage contracted before 1870, while for marriages contracted after 1870 , the average was 3.08 . 
In considering the effect of differential birth rates it is desirable to know how many children per family are required to maintain a stock without loss. It is obvious that more than two are required because, in addition to the two children who will replace their parents, others are needed to supply the places of those who die before reaching maturity and those who fail to marry. Evidently the number of children needed depends upon the death rate and the marriage rate. In his celebrated pronouncement on race suicide Theodore Roosevelt estimated that, on the average, four children per marriage are needed to keep a stock from decreasing in numbers. With the reduction of infant and child mortality which has occurred since this statement was made, the required number of children is now somewhat less. According to the calculations of Dublin it is about 3.I. Estimates made in Germany have given 3.3 (Grassl I9I4); 3.2 (Burgdörfer, I924-1926), and 3.4 (Lenz I924-1926) as the required numbers under prevailing rates of marriage and mortality. In general it may be said that stocks producing less than 3 children per family will decrease in numbers in successive generations.

With this fact in mind it is instructive to compare the birth rates of different social and occupational groups. There have been several studies of the families of the graduates of colleges and universities, and they agree in showing that the birth rate in this class has declined to a very low point. Records for Harvard give an average of I.9 children per marriage of graduates, or, allowing for the 18.9 per cent who are not married, I.6. For Yale graduates the corresponding figures $(1893,1896,1897)$ are 1.9 and I.5; and for the graduates of Swarthmore College (1896I9I2) they were 2.I 5 and I.9. The records for graduates of women's colleges are considerably lower than those from men's colleges because of the smaller percentage who marry. 


\section{I8 HUMAN GENETICS AND ITS SOCIAL IMPORT}

The percentages of married graduates of Vassar have varied from 53 to slightly over 70 , and the number of children per marriage from 2.4 to 1.9 , and the number per graduate from I.5 to I. The percentage of women graduates of the University of California who married between 1872 and 1910 inclusive is 55 . For most women's colleges the percentage of graduates who marry is probably between $5^{\circ}$ and 60 . The low birth rate of the graduates of women's colleges is probably due not so much to the influence of college education as to the mores of the group to which the college girls belong. Mary Roberts Smith found that 343 married college women had 566 living children, or 1.65 per marriage, while $3^{\mathrm{I}} 3$ noncollege women of the same social class-sisters, cousins, or friends-had 582 living children, or 1.87 per marriage. The small difference is probably due to the fact that the college women married about two years later than the group which did not attend college. Similar findings have been reported by Goodsell.

The studies made on the number of children born to college graduates make it evident that, on the whole, the fertility of this group falls distinctly below the maintenance level for men, and very far below for women. A similar situation is afforded by other classes distinguished for their intellectual achievements. Cattell finds that the average number of children in the families of American men of science is r.88. The people whose names get into Who's Who in America have families averaging little over two children. Huntington finds that 384 faculty members of Yale University who were born before 1890 have on the average 1.65 children. Although some of the younger members may have more children, they will be few in number and probably would not bring the average up to I.8. Other studies indicate that much the same situation prevails in other institutions. The families of professional people in 
general-lawyers, doctors, engineers, teachers, and investigators-the people who are especially distinguished on the basis of intellectual training, are now reduced below the level necessary for continuous propagation. The kind of heredity which these stocks represent is, therefore, being lost to the race.

To a great extent the members of these classes come from the larger class of college graduates. The latter come mainly from a fairly intelligent, more or less prosperous stratum of society who are appreciative of the value of education at least to the extent of sending their children to college. Such people would be a serious loss to the community. Do they constitute a self-perpetuating group?

I have endeavored to answer this question, so far as the parents of the students in the University of California are concerned, by ascertaining the number of children in the families from which these students came. The average number based on the parents of over 16,000 students was 3.66 per family. One might consider that this number is sufficient for maintenance, and so it is for these particular families. But before concluding that the general stratum of which these parents are a fair sample is sufficiently fertile to perpetuate itself, we must take into consideration a source of error which has often led to false conclusions in the field of eugenics. It arises from the fact that if we start with any group chosen at random from the general population, whether school children, inmates of sanatoria or asylums, and then ascertain the size of the families from which these individuals came, the families will be larger than the general average of their kind. Families having no children would obviously not be represented at all. Families with ten children would be represented ten times as frequently as families with one child. Consequently our group would be especially selected for large family size. 


\section{HUMAN GENETICS AND ITS SOCIAL IMPORT}

If we find that insane, feeble-minded or tuberculous children in an institution come from families that are larger than the average, it does not necessarily follow that the stocks which furnish them are unusually prolific. They may or they may not be, but in order to ascertain whether they are or not, we must compare averages obtained in the same way. By knowing the numbers of children in the several families constituting a given group, we can calculate the extent of the bias in favor of large families and estimate the true average of the group from which it was chosen, provided the choice represents a true random sample. By making such an estimate, and considering only fertile marriages, the average size of the families represented in our University of California group would be reduced from 3.66 to less than 3 . If we make further allowance for childless marriages, which in such a group probably average about 15 per cent, the number would be reduced still more.

In a similar study made by Baber and Ross on families sending students to the University of Wisconsin, it is found that the average size of the family was 3.76 , and that the families of the uncles and aunts of the students average 3.24. The families of the latter group were not weighted for large size, but they included no sterile marriages. The fact that parents of college students have larger families than their brothers and sisters is probably due mainly to the fact that the families were selected for large size. This tendency would be partly counteracted by the circumstance that the parents of a small family might better afford to send a child to college than parents of a large family. On the whole, it seems probable that the stratum of people who send students to college fall somewhat short of being a self-perpetuating group.

This conclusion is in harmony with the data on the relative birth rates among members of different occupations. 
Occupational fertility has been studied in Great Britain, Germany, and some other European countries and more recently in the United States. The findings tell much the same story. One of the best investigations has been carried on in England by Dr. T. H. C. Stevenson based on the census of IgII. Occupations were classed in eight groups. The first five includes workers arranged according to status from the professional and upper classes (group I), to unskilled workers (group V). The sixth, seventh and eighth groups comprise textile workers, miners, and agricultural laborers respectively. The relative standardized fertility rates per hundred families of these groups are shown in the table, as well as their trend since about the middle of the century.

Table 9.-Children Born per ioo Families in England and Wales According to Occupational Groups and Date and Duration of Marriage.

Standardized Rates

\begin{tabular}{|c|c|c|c|c|c|c|c|c|c|}
\hline \multirow{2}{*}{$\begin{array}{l}\text { Date of } \\
\text { marriage }\end{array}$} & \multirow{2}{*}{$\begin{array}{c}\text { Duration of } \\
\text { marriage }\end{array}$} & \multicolumn{8}{|c|}{ Occupational class } \\
\hline & & I & II & III & IV & $\mathrm{V}$ & VI & VII & VIII \\
\hline Igo6-IgII & $0-5$ & 70 & $8 I$ & 86 & 90 & 100 & 76 & 105 & IOI \\
\hline 190I-1906 & $5^{-10}$ & 171 & 197 & $2 I I$ & 219 & $24^{2}$ & 185 & 263 & 246 \\
\hline I 896-I IOI & $10-15$ & $24^{2}$ & 284 & $3^{I} 4$ & 323 & 362 & 275 & 399 & 363 \\
\hline $189 I-1896$ & $15-20$ & $3 \circ 3$ & 359 & 405 & 412 & 463 & 359 & $5^{17}$ & 470 \\
\hline I 886-I 89I & $20-25$ & 357 & 422 & 482 & $49^{1}$ & $54 \mathrm{I}$ & 435 & 610 & $55^{2}$ \\
\hline I $88 \mathrm{I}-1886$ & $25-30$ & $4^{1} 3$ & $48 \mathrm{I}$ & 544 & $55^{\circ}$ & 596 & 501 & $67 \mathrm{I}$ & 618 \\
\hline $187 I-I 88 I$ & $30-40$ & 497 & 567 & 615 & 616 & 652 & 567 & 717 & 667 \\
\hline $186 I-I 87 I$ & $40-50$ & 607 & 665 & 696 & 690 & 715 & 648 & 777 & 719 \\
\hline \multirow[t]{3}{*}{$185 \mathrm{I}-\mathrm{I} 86 \mathrm{I}$} & $50-60$ & 662 & 733 & 746 & 735 & 763 & 696 & 797 & 779 \\
\hline & Over 60 & 682 & 777 & 729 & $79^{2}$ & $78 \mathrm{I}$ & 732 & 870 & 820 \\
\hline & Total.. & 277 & $3^{21}$ & 353 & 359 & $39^{2}$ & 319 & 433 & 399 \\
\hline
\end{tabular}

It may be noted that the fertility in the professional group is very low and that there is an increase as we pass to the lower occupational groups. Moreover, if we compare the differences in fertility between the lower and the higher groups, we find that they are much less pronounced in 


\section{HUMAN GENETICS AND ITS SOCIAL IMPORT}

marriages occurring between $185^{\mathrm{I}}$ and $\mathrm{I} 86 \mathrm{I}$ than in those taking place at a later date.

In Prussia in I9I2, according to Lenz, there were the following numbers of children per marriage in the classes listed:

Table 10.-Children per Marriage in Different Classes in Prussia in igi 2

Number

Occupation

of Children

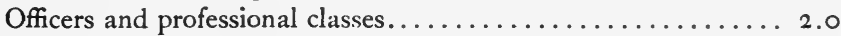

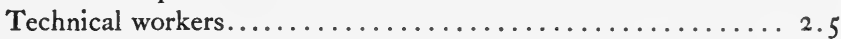

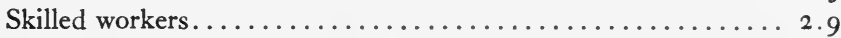

Factory workers without technical training............. 4

Agricultural and day laborers................. 5.2

Statistics on the size of the families among followers of various occupations in the United States have been tabulated

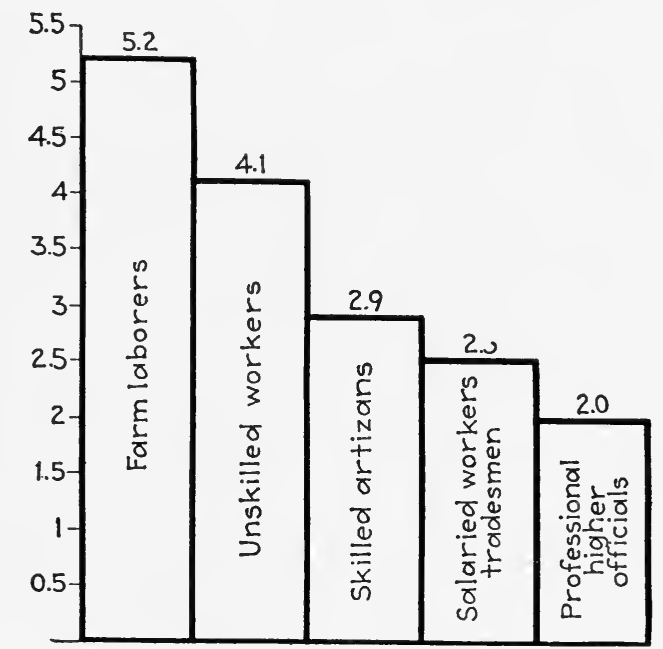

FIG. 63.- Children per marriage in Prussia in 1912 according to occupation.

in our volumes on birth statistics for a few years since 1924, and data on the subject were collected, but not published, for the Census of Igio. According to Sydenstricker and Notestein the number of children per 100 wives in a large sample of a population in IgIO were as follows: 
Table il.-Number of Children per io Wives in Selected Areas of the UNITED STATES

\begin{tabular}{|c|c|c|}
\hline \multirow[b]{2}{*}{ Area and class } & \multicolumn{2}{|c|}{ Number of children } \\
\hline & $\begin{array}{c}\text { Mothers of all ages } \\
\text { at marriage }\end{array}$ & $\begin{array}{c}\text { Mothers aged } 20-24 \\
\text { at marriage }\end{array}$ \\
\hline \multicolumn{3}{|l|}{ Urban sample: } \\
\hline Professional.. & I $5 \mathrm{I}$ & $\mathrm{I}_{4} 8$ \\
\hline Business............... & 152 & 146 \\
\hline Skilled workmen........... & 178 & 170 \\
\hline Unskilled workmen....... & 213 & 206 \\
\hline \multicolumn{3}{|l|}{ Rural sample: } \\
\hline 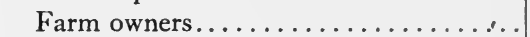 & 233 & $22 I$ \\
\hline 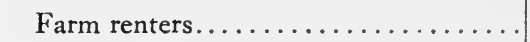 & $25^{8}$ & 248 \\
\hline 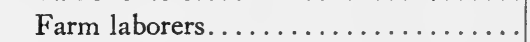 & 277 & 253 \\
\hline
\end{tabular}

The age of wives at marriage varied in these groups, being about five years later in the professions than among the

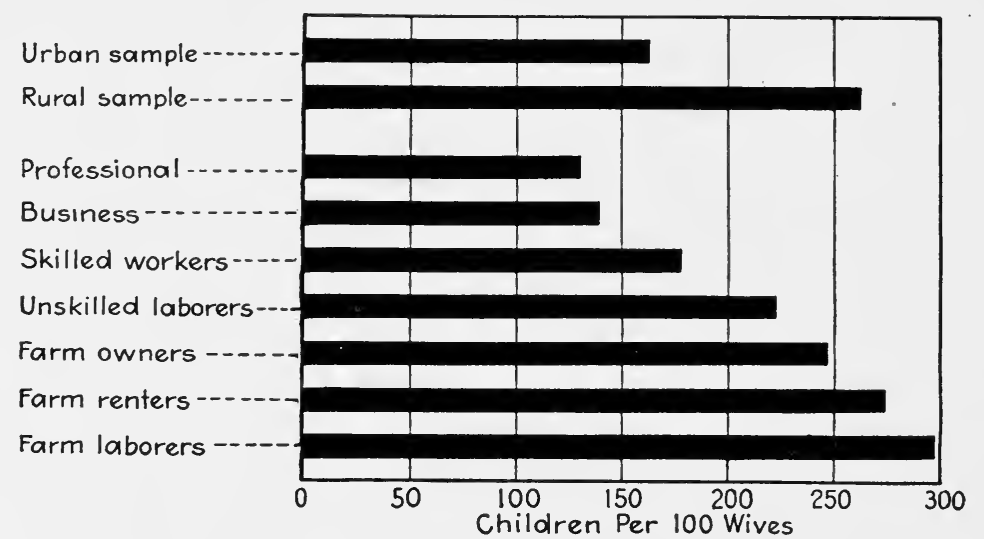

Fig. 64.-Children born to roo wives according to occupation of husband in selected areas of the United States in 1910. (Data from Notestein.)

farm laborers and over four years later than in the unskilled workers. However, for wives marrying at any particular age, such as twenty to twenty-four for instance, the same occupational trend is shown as in the whole group. It is significant that the percentage of wives forty to forty-nine 


\section{HUMAN GENETICS AND ITS SOCIAL IMPORT}

years of age who remained childless increases as the birth rate declines, while the percentage who have had five children or more is fairly parallel with the occupational trend of the birth rate.

Table 12.-Percentage of Childless Wives and the Percentage of Wives Bearing Five or More Children According to Area and Social Class

(Ages of wives forty to forty-nine)

\begin{tabular}{|c|c|c|}
\hline Area and class & $\begin{array}{l}\text { Percentage } \\
\text { childless }\end{array}$ & $\begin{array}{c}\text { Percentage with } \\
\text { five or more children }\end{array}$ \\
\hline Urban. & 15.7 & 16.9 \\
\hline Professional..... & 17.7 & 10.9 \\
\hline Business......... & 16.0 & 12.4 \\
\hline Skilled workers... & 15.1 & 21.5 \\
\hline Unskilled workers. & 14.0 & 33.6 \\
\hline Rural............ & 9.2 & 39.0 \\
\hline Farm owners... & IO. I & 35.2 \\
\hline Farm renters... & 6.8 & 49.2 \\
\hline Farm laborers....... & 7.0 & 50.2 \\
\hline
\end{tabular}

The inverse relation between fertility and occupational status; according to Kiser, has increased between 1900 and I9IO, at least in the East North Central states. Ogburn, who has compared the sizes of families in relation to occupation between I900-1930, states, "The greatest decline was among the families of the professional group where it was Io per cent." The data from the United States agree with those from England and Wales in showing that differences in the fertility rates of occupational classes have been increasing. These differences are now very marked, and they show that in general the birth rates increase in proportion as the occupations require little intelligence or skill.

F. Lenz, T. Lenz, and K. Barries in studying over 3,000 children in the schools of Kronach find that the average number of children per family varies inversely with scholastic grades and levels of intelligence. The scholastic records and number of children per family were as follows: 
Table I3.-The Relation of Scholastic Record and Ability to Family Size IN KRONACH

$\begin{array}{cc}\text { Scholastic record } & \begin{array}{c}\text { Number of children } \\ \text { per family }\end{array} \\ \text { I } & 3.30 \\ 2 & 3.47 \\ 3 & 3.81 \\ 4 & 4.24 \\ 5 & 4.11 \\ \text { Rating for ability } & \\ \text { I } & 3.28 \\ 2 & 4.03 \\ 3 & 4.43 \\ 4 & 5.05 \\ 5 & 5.15\end{array}$

S. Dawson's studies of the relation between intelligence quotients and family size in a group of 1,239 children of workmen and tradesmen in England showed that in the highest group with an I.Q. of II 4 and over the average was 3.4I; for the medium students (I.Q.'s $85^{-I_{1}} 4$ ) it was 4.43, and for the dull (I.Q. less than 85 ) it was 4.73. The correlation between intelligence and family size was -0.30 . Studies by Bradford on $45^{\circ}$ city children gave a correlation of -0.35 , and those of Chapman and Wiggins on 670 children a correlation of -0.33 .

From the biological point of view these facts would have little significance if the differences between the groups represented have no relation to heredity. If the distribution of intelligence is essentially the same in all social and occupacional groups, it matters little, so far as the inherited qualities of the race are concerned, which groups produce the greatest number of children. Socially and in other ways it might make a good deal of difference of course, but whether the differential birth rate works dysgenically or not depends upon whether the classes characterized by different degrees of fertility differ also to some extent in their genetic endowments. This question, which we have discussed in a previous chapter, is one of crucial importance, and, in considering it, 


\section{HUMAN GENETICS AND ITS SOCIAL IMPORT}

we should free ourselves from all forms of class bias which have a strong influence in determining the attitudes of people on this subject. Perusal of the controversial literature on eugenics will reveal the existence of two kinds of class prejudice which may be described as the aristocratic and the proletarian. According to the first, occupancy of a lowly station in life is ipso facto an evidence of innate inferiority. According to the proletarian bias, the marks of inferiority which may be associated with a lowly station in life are due to the unjust distribution of wealth and opportunity in our present social order. Those who are actuated by the latter bias are prone to resent the conclusion that the differential birth rate in relation to social and occupational class is in any way dysgenic. To assert that ordinary unskilled laborers have an I.Q. lower than skilled artisans or successful business men is regarded as adding insult to injury. Superior occupational status and especially financial success is attributed not so much to superior intelligence as to the accidents of fortune or to a selfish acquisitiveness which facilitates the attainment of success in the scramble for wealth and position. Hence the conclusion that the present differential birth rate is dysgenic is by no means lacking in vigorous opponents. Commonly the opposition is based on some of the following assumptions:

I. That intelligence tests and even scholastic records are no measure of native ability. This position, although seldom adopted in the bald form stated, we have discussed in a previous chapter.

2. That superior intelligence, even though inherited, is so dependent upon the fortunate concourse of genes in the Mendelian shuffle that it is about as apt to arise from stupid parents as from intelligent ones. Hence the differential birth rate does little harm. At least an approach 
to such a position has been made by Dr. H. S. Jennings, who, while admitting the great importance of heredity, has stated that "superior parents often produce mediocre or inferior offspring; inferior parents at times produce mediocre or superior offspring. In consequence of this situation, decrease or even stoppage of the superior individuals or of the inferior individuals has very little effect on the average grade of the next generation."

3. That inherited levels of intelligence make no difference in occupational status. If a rigid caste system prevails in which sons follow the footsteps of their fathers, this might possibly be true. But in most countries at present, and in our own in particular, there is a great deal of change from one occupational level to another, both upward and downward. Does native ability count for nothing in determining these changes? Common observation, to say nothing of other evidence, indicates that it is an important factor in what is commonly called success. It is not the dull people in the lower levels who rise to superior status. The moron, borderline, and dull-normal groups naturally gravitate into the ranks of unskilled and relatively poorly paid labor. That on the whole the intellectual levels below mediocrity commonly get into pursuits which require an unusual mental equipment is hardly a reasonable conclusion.

4. That the average differences between occupational groups are not great enough to indicate that they cannot be entirely accounted for as a result of educational advantages and opportunity. It must be admitted that a part of the differences can be so explained, but that the explanation is entirely adequate is open to the objections stated in the previous paragraph.

5. That the sterility of the upper classes can be continually supplied from below while the lower strata continue to reproduce their own kind. 
Opposed to these interpretations most eugenists would contend:

I. That human beings manifest different degrees of intelligence which to a considerable extent are dependent upon differences in genetic constitution.

2. That the higher occupations more than the lower ones are followed by individuals who have the good luck to have drawn a favorable combination of genes.

3. That the children of parents in these higher groups owe at least a part of their superiority in intelligence quotients and scholastic records to their somewhat superior heredity.

4. That the sterility of the higher groups represents a loss to the race of the more highly endowed hereditary types.

5. That the rise of people from the lower occupational groups into the higher ones where they become relatively sterile tends to rob the lower groups of the good hereditary material they contain, leaving them to be recruited more and more from the less well endowed members who fail to rise. Thus the tendency of society to "die off at the top" results in the impoverishment of our racial heredity as a whole.

6. The differential birth rate under present conditions is on the whole dysgenic in its effects.

There are, of course, all sorts of intermediate positions between the opposed standpoints we have mentioned. The student will have to draw his own conclusions as to what position is the most reasonable in the light of the available evidence.

The negative correlation between fertility and intelligence as it is measured by different kinds of tests is what one might naturally anticipate, because people with little intelligence would not be likely to exercise prudence and foresight in regard to reproduction any more than they do 
in other affairs of life, if as much. Birth control involves a measure of sophistication and at present it is employed relatively little by unsophisticated people. Although the practice at first prevailed in the higher social strata, it has gradually filtered down through the ranks of society until, in several regions, it has caused a marked decline of the birth rate in the proletariat. In fact, were it not for the participation of this numerous class, the general birth rate could not have fallen as much as it has in several countries. Birth restriction has been much more extensively employed by the proletariat since the World War, especially in Germany where the notion of a birth strike (Geburtstreik) has become widespread among the industrial workers. Some students of vital statistics are convinced that the differential character of the birth rate is decreasing because contraceptive practices have invaded the lower social and economic levels. In Berlin there is little difference between the birth rates of the proletarian and the well-to-do districts. In Hamburg in IgIo the birth rate was about twice as high in the proletarian sections as in those with a prosperous population, but from I926 to I930 the differences were very much reduced. Similar trends have been recorded for Dresden and Königsberg. Both Stockholm and Oslo showed high birth rates in the poorer districts before the war (I9II-I9I4), but the decline has been so rapid in the poorer districts that in I927-I928 it was very little higher than in the rich districts. In London and Edinburgh the same tendency is manifest, but it has not proceeded so far. In Paris the poor districts were much more fertile than the rich ones from I 906 to I 9 IO (in some cases nearly twice as fertile), but since I 925 the differences have been very much less.

We have few data on how the differential birth rates have been changing in cities of the United States and in most other countries. It is safe to say that in most places 


\section{HUMAN GENETICS AND ITS SOCIAL IMPORT}

they have not as yet gone very far toward equalizing the birth rates of different social classes. What has happened in the places where the inequalities of the birth rate have been largely effaced is not that the fertility of the higher social classes has increased (generally it has slowly decreased), but that it has decreased so much in the lower social classes that the total birth rate has come to be below the level required for maintenance. Were the present rates to continue all classes would become extinct together.

Probably the general trend now is toward the equalization of birth rates at a lower level. As this movement progresses the dysgenic working of differential fertility will be reduced and may eventually be followed by a greater fertility of the successful types. It has been suggested that the present dysgenic period of our biological history is only a temporary stage which will be passed through as the practice of birth control has completed its downward course throughout the masses. We may at least cherish this possibility as a pious hope. But with this must be joined another pious hope that, before this happy consummation is reached, the population will not have begun to decrease in numbers.

While there is a pronounced tendency for birth rates to rise as our measures of intelligence indicate that intelligence quotients are low, there are some exceptions to this rule. Butt and Nelson have found that in two counties in Utah parents with more than the average schooling have slightly larger families than those with less schooling. The parents were mostly farmers, largely of the Mormon faith, and constituted a fairly homogeneous group. In a rural section of Vermont, Conrad and Jones have found that there is practically no relation between intelligence of parents and family size. Here again there was a fairly homogeneous rural population like the Utah farmers which still retained the older traditions of high fertility. 
Exceptions of a somewhat different kind have been reported by Huntington and Whitney, who found that among the 800 Yale graduates of the classes of I893, I896, and 1898 the men who were graded high by their colleagues on the basis of their general value to society had, as a rule, larger families than the graduates who were graded low. The men were classed in ten groups according to the degree of success they had attained. The percentage who were married increased with the degree of success and the number of children per father increased from 2.2 at one extreme to 3.I at the other. Similar relationships were found by J. D. Phillips for graduates of Harvard, and by F. A. Woods for individuals in Who's Who in America. Huntington remarks, "No matter whether we take lawyers, ministers, engineers, bankers, artists, military men, writers, business men, or professors, the successful men on the average have decidedly more children than the less successful."

All these groups, however, are characterized by a reproductive rate that falls below the maintenance level, so the tendency noted represents a sort of back eddy in the general stream. Dr. Huntington suggests that "the distribution in families arising from birth control has run its course among the distinguished men and that they are returning to a normal situation wherein they will have enough children to replace themselves and to provide for a small increase from generation to generation," - a sentiment to which the eugenist would say Amen.

In considering the fertility of the people of low intelligence we may dismiss the very lowest types, the idiots and imbeciles, as practically negligible from the standpoint of eugenics. They are relatively few in number, and, as they are usually kept under surveillance in their homes or in institutions, they rarely produce offspring. With the morons it is a different matter. Stocks like the Jukes, Kallikaks, 


\section{HUMAN GENETICS AND ITS SOCIAL IMPORT}

and Tribe of Ishmael flourish like the green bay tree, and there are many records of the unrestrained prolificacy of feeble-minded women, as is illustrated by the reproductive performances of Polly and her eleven illegitimate children who were responsible for $3 \mathrm{I}$ feeble-minded progeny among their 56 descendants. However, one should not base conclusions on selected cases. What we are interested in knowing is whether the moron group as a class is more prolific than persons of normal intelligence.

Unfortunately, there are only a few scientific studies on the birth rates of the feeble-minded. Most mental measurements are made upon children instead of parents. In one investigation made by Green on family size in 2 I I feebleminded parents, it was found that the average number of children was $6.43 \pm 0.17$. These results were obtained from records of family histories in the Eugenics Record Office at Cold Spring Harbor, N. Y. The families may have been somewhat larger than normal on account of the method of selection, but only a small proportion of the women studied were mothers of the individuals on the record charts. It is significant that there was almost no decrease in family size since I840. In the Eugenics Survey of Vermont, it was found that where one or both parents were feebleminded or insane the average size of the family was 3.5 , or including still births and children dying in infancy, 4.3-figures somewhat larger than those of normal families (3.04 and 3.34).

Popenoe reports that II 2 feeble-minded mothers of inmates of the Sonoma State Hospital in California had an average of 4.09 children (4.95 pregnancies), which when corrected for selection was reduced to 2.84 (3.I7 pregnancies); but, on account of the age of the mother, these families were not quite complete. This fertility is certainly not excessive even for a state with a low birth rate such as California. 
More is known about the fertility of parents of mental defectives than about the fertility of defectives themselves. According to the report of the Mental Deficiency Committee of Great Britain, parents of very low grade mental defectives have families of about the average size. Since, as we have seen in a previous chapter, such children are nearly as apt to come from people in one intelligence level as in another, this is perhaps to be expected. The same investigators have found, however, that the parents of mental defectives of a higher grade are characterized by an unusually high birth rate. This also might be expected if there is a negative correlation between intelligence and fertility, since defectives of this class are, as we have seen, more commonly derived from parents of a relatively low mental level. Similar results are reported by Thurstone and Jenkins who find that in Chicago, idiots and imbeciles with an I.Q. of less than 40 come from families of about the average size, while defectives with I.Q.'s from 50 to 80 come from families about one-third larger than those with children of normal or superior intelligence.

In his investigation of the families of I, III mentally defective children in Liverpool Dr. Caradog Jones found that the average size of families containing one or more defective children (age five to twenty-two) was 7.2, of whom 2.5 had died, leaving 4.7 living children per family. The average size of a random sample of 4,379 working-class families in Liverpool was 3.92 children born, and 2.97 living children. Even allowing for the different basis of selection in the two cases the facts support Dr. Jones' conclusion that, in Liverpool at least, "the subnormal group generally is contributing considerably more than its fair share to the future population." To a very considerable extent mentally defective children in Liverpool come from the lower occupational groups. In a sample of families found to contain 


\section{HUMAN GENETICS AND ITS SOCIAL IMPORT}

at least one mentally defective person in a household census of the general working-class population, the head of the family was unskilled in 63 per cent of the cases as compared with 39 per cent of unskilled laborers in the general survey. In one-fourth of the cases the head was unemployed as compared with one-tenth in the general sample; and over 40 per cent were below a defined poverty line as compared with I6 per cent of all the families sampled in the same area.

Most of our data on the fertility of families producing mental defectives come from studies based on children in special schools or classes. Dr. L. J. Whitney reports that the average size of the family of I,63I children in schools for subnormals in an eastern city was 7.09. To a large extent the children came from recent immigrant stocks, and the families were probably larger than the average for this reason. Keller, in studying the relations between family size and defective intelligence in Gross-Winter, Germany, finds that children in special classes for defectives came from families averaging 4.47 , while the number of children per family in the primary schools was 4.I5, and in secondary schools $3.3 \mathrm{I}$. If these figures are corrected for the effects of selection they would be $3.25,2.98$ and 2.46 respectively. The figure for the special class children is too low because their ages were from 6 to I4, and hence more children will probably be born in these families. In the other cases the families were more nearly completed, since the children were older, being from I 2 to I 5 years of age. In a similar investigation in Mecklenburg, Winkler finds that the fertility of families in special schools for defectives is "about one-fourth higher than in those of more than average mentality." In Bremen Kurz finds that children in special schools came from families averaging 4.3 children while the average for high schools was 2.10. 
One of the most extensive studies on the parentage of defective and retarded children has been carried on by Dr. Neil A. Dayton who made a survey of 20,473 such children in the public schools of Massachusetts. These children were found to come from families averaging 5.6. This number reduced for selection of families of large size becomes 3.9, which is somewhat larger than the estimated size of a completed family in the United States in 1920, namely 3.6. The larger size of the Massachusetts families is attributed by Dayton to the high percentage of foreign-born parents in the group examined. Making allowance for this factor brings the average down to 3.5 which is not far from the average family size in the entire country. Children with an I.Q. less than 70 were classed as defective; those with I.Q.'s from 70 to 89 were called retarded. Unfortunately the family size of the normal children in the same schools was not determined, since this would have furnished valuable data for comparison drawn from families in the same communities. How far the sample studied is representative of the general population as to urban and rural distribution is uncertain. The average age of the mothers was about forty-one, and hence Dr. Dayton concludes that the families were practically completed. However, the percentage of children born to mothers over forty in Massachusetts has commonly been about five per cent, but if the average age of the mothers was forty-one there must have been many mothers younger than this to counterbalance those of older ages, so it is not quite safe to assume that the families were practically complete. At any rate, even if Dr. Dayton's estimates of family size are somewhat too low, the fertility of most of the parents sending defective and retarded children to the public schools of Massachusetts does not greatly exceed that of the general average of the American people. Certainly it is more than sufficient for the main- 


\section{HUMAN GENETICS AND ITS SOCIAL IMPORT}

tenance of this group, and doubtless considerably exceeds the fertility of the families in that state which send their children to college.

On the whole the evidence points to the existence of a negative correlation between intelligence and fertility for all grades of intelligence from the highest down to but not necessarily including the lowest type of mental defectives. There can be no doubt that in most countries of advanced culture, the higher occupational groups are not perpetuating themselves. With the general decline of the birth rate inadequate reproduction has gradually proceeded downward until, in several regions, the only groups which are maintaining themselves are those in the lower levels. Conditions vary considerably in different countries and in different areas especially urban and rural in the same country, but the tendency for high intelligence to be associated with low fertility is very widespread in Europe and America.

\section{Suggested Readings}

Charles ('34). Holmes, ('21), chaps. 6 and 7. Huntington and Whitney ('27), chaps. 4-6, I5-20. Jones, D. C. ('32). Ogburn and Tibbitts ('32). Wiggam ('24), chaps. I8, I9.

\section{Questions}

I. How is the quality of the birth rate affected by the feminist movement, the celibacy of the priesthood, the decrease of illegitimacy?

2. Why does the average person come from a family of more than the average size?

3. Why do great men come from predominantly boy-producing families, and great women from predominantly girl-producing families?

4. Eminent persons are more apt to occur among the first-born children of a family. Are the reasons for this genetic or cultural ?

5. Why does Utah have a high birth rate?

6. Do you think that in the future birth control will be eugenic or dysgenic?

7. What are some of the involuntary and some of the voluntary causes of the decline of the urban birth rate? 
8. Why has the rural birth rate declined with especial rapidity in the last few years?

9. Look up in the United States birth statistics the family size of the different national groups. What relation has fertility to the cultural and economic status of these groups?

Io. Look up the family size of various occupational groups in the United States birth statistics. What groups are above and what groups are below the maintenance level?

II. What are some of the purely statistical reasons for a decline in the birth rate, and what are the more important real reasons for the decline?

I2. If people reduce their birth rate for economic reasons, why is the birth rate lower in well-to-do areas than it is in poorer ones?

13. In studying the influence of religion on the birth rate, what sources of erroneous inference should be considered?

14. What is the average size of the family in the class to which you belong? Does this indicate a birth rate sufficient for maintaining the stock under existing rates of death and marriage? 


\section{CHAPTER XVIII}

\section{DEATH RATES}

TN ORDER to ascertain how rapidly a population increases in numbers it is necessary to have records of the rate at which people die as well as the rate at which they are born. The measure of mortality most commonly employed is the number of individuals dying during the year per thousand population of that year. This is called the crude death rate. And its interpretation is beset with all the sources of error to which attention has been called in discussing the crude birth rate. Since people are more apt to die at some ages than at others the general death rate of a country is influenced, like the birth rate, by the age composition of the inhabitants. A community composed of very young or very old people, for instance, would have a high mortality since the death rate in both these age groups is higher than in the middle period of life. If the local newspaper or Chamber of Commerce boasts of the low death rate of one's town it does not necessarily indicate that the locality is a particularly healthful one in which to live, because the town may contain a large number of young workers or college students whose low death rate reduces the general average.

In order to make proper allowance for differences in age composition it is customary to employ standardized death rates obtained by reducing all death rates to a common basis. This is done by finding what the rates of different regions would be if their populations had the same age composition as a given population taken as the standard of 238 
comparison. One standard commonly employed is the population of England and Wales in I90I. To calculate the standardized death rate of a region one needs to know the death rate for each age period. Then one can estimate how many deaths would have occurred in each period had the age composition of the inhabitants been the same as that of the standard population. When this is determined one may calculate how many deaths per thousand would have occurred in the total population of the region.

The choice of a population as a standard is a purely arbitrary proceeding, and the populations of different countries and at different times have been used for this purpose. Sometimes mortality rates are further adjusted for non-residents, inmates of hospitals, or other persons not typical members of the community.

Mortality records have not been systematically kept over a long period of time. What is known about the mortality of people in past centuries has to be gathered from the records of ages at death that happen to have been preserved, and these are very incomplete. Records of baptisms, marriages, and deaths were first kept in England and France in the sixteenth century. In 1662 John Graunt of London published the first treatise on vital statistics entitled Natural and Political Observations Mentioned in the Following Index, and Made upon the Bills of Mortality. Graunt brought out several significant facts concerning mortality, such as the relatively high death rate of males, the high death rate in infancy, and the greater mortality of urban than of rural inhabitants. His work afforded a stimulus to later studies in the same field, but for a long time progress in recording vital statistics was slow. Only a few countries published adequate mortality statistics before the nineteenth century, and there are still many parts of the earth in which no records of deaths are kept. 


\section{HUMAN GENETICS AND ITS SOCIAL IMPORT}

For a long time information on the mortality of the people of the United States was very incomplete. A few states and cities have published fairly satisfactory reports on deaths for many decades, and data on mortality for the entire country have been compiled at ten year intervals in the reports of the Census, but they are confessedly very inaccurate. Since 1900 the Census has issued annual volumes on mortality statistics for the Registration Area for Deaths. This area, in which the registration of deaths is fairly complete, has gradually increased from a few states until it now includes all the states of the Union. Similarly the Registration Area for Births has also increased until it comprises the entire country. As a result of these improved facilities for biological bookkeeping, changes in population can be followed much more accurately than was possible a few years ago.

Reliable mortality statistics are quite evidently necessary for the construction of that very useful compendium of information about life and death known as a life table. Sometimes the same thing is called a mortality table, but either name is appropriate, since the table gives us information concerning both probable further duration of life and chance of dying within the year at any given age. Life insurance rates are based on life tables, and since one's expectation of life decreases as one gets older, a policy taken out after age forty costs more than one begun earlier in life. If a man is over age sixty-five most companies will not insure him at all.

If taking the data supplied by a life table, we construct a curve representing the mortality of human beings at different ages, we find that it takes a peculiar course. Erecting perpendiculars at each age along a base line at heights which are proportional to the death rates at these ages, we see that our curve starts at a high point because the first year 
Table 14.-Life Table for Males and Females in Illinois, 1929-1931, According to Computations of the Metropolitan Life Insurance Co. (The probable expectation of life and death rates are given for the several ages.)

\begin{tabular}{|c|c|c|c|c|}
\hline \multirow[b]{2}{*}{ Age } & \multicolumn{2}{|c|}{ Males } & \multicolumn{2}{|c|}{ Females } \\
\hline & $\begin{array}{c}\text { Exp. of } \\
\text { life }\end{array}$ & $\begin{array}{l}\text { Death } \\
\text { rate }\end{array}$ & $\begin{array}{l}\text { Exp. of } \\
\text { life }\end{array}$ & $\begin{array}{l}\text { Death } \\
\text { rate }\end{array}$ \\
\hline 0 & 59.02 & 60.30 & 62.82 & 46.28 \\
\hline$I$ & $61 \cdot 78$ & 9.13 & 64.84 & $8.0 \mathrm{I}$ \\
\hline 2 & $61 \cdot 34$ & 5.23 & $64 \cdot 3^{6}$ & $4 \cdot 7 I$ \\
\hline 3 & 60.66 & 4.22 & 63.66 & $3 \cdot 4^{6}$ \\
\hline 4 & $59 \cdot 9^{I}$ & $3 \cdot 35$ & 62.88 & 2.95 \\
\hline 5 & 59. I I & 2.84 & 62.06 & $2.5^{2}$ \\
\hline 10 & $54 \cdot 78$ & 1.72 & 57.63 & $I \cdot 3^{I}$ \\
\hline 15 & 50.24 & 2.09 & 53.00 & 1.70 \\
\hline 20 & 45.82 & 2.95 & $4^{8} \cdot 5^{2}$ & 2.55 \\
\hline 25 & $4^{I} \cdot 5^{I}$ & $3 \cdot 37$ & 44.16 & 3.08 \\
\hline 30 & 37.21 & $3 \cdot 9^{2}$ & 39.85 & $3 \cdot 5^{2}$ \\
\hline 35 & 32.97 & 5.21 & $35 \cdot 55$ & $4 \cdot 13$ \\
\hline 40 & 28.89 & $7 \cdot 17$ & $3^{1} \cdot 3^{2}$ & $5 \cdot 34$ \\
\hline 45 & 24.98 & 9.78 & 27.21 & 7.20 \\
\hline 50 & 21.26 & 13.40 & 23.24 & $10 . \infty$ \\
\hline 55 & 17.77 & 19.23 & 19.49 & 14.60 \\
\hline 60 & $14 \cdot 5^{8}$ & $27 \cdot 48$ & 16.01 & $21 \cdot 39$ \\
\hline 65 & I I . 70 & $40.0 \mathrm{r}$ & 12.83 & $32 . \infty$ \\
\hline 70 & 9.18 & $5^{8.25}$ & 10.02 & $4^{8} \cdot 31$ \\
\hline 75 & 7.02 & 86.55 & 7.60 & $74 \cdot 17$ \\
\hline 80 & $5 \cdot 29$ & 128.55 & 5.66 & II 6.67 \\
\hline $85^{\circ}$ & $3 \cdot 9^{8}$ & 183.43 & 4.23 & $167.6 \mathrm{I}$ \\
\hline 90 & 3.02 & 249.88 & 3.06 & 234.74 \\
\hline
\end{tabular}

of life is a very hazardous period. Then the curve slopes downward quite rapidly, and afterward more slowly through the years of childhood and reaches its lowest point about age eleven. After this people begin to experience the effects of really growing old. The death rate rises slowly through the teens, more rapidly in middle life, and still more rapidly in old age, until in the nineties it shoots upward with a steep ascent. Curves of mortality vary with time and place, but however high or low the general death rate may be, they always preserve the same general form. 


\section{HUMAN GENETICS AND ITS SOCIAL IMPORT}

Deaths are due to both hereditary and environmental factors, but the main features of the mortality curve are the product of internal causes. We are like clocks constructed to keep going for a certain length of time. Accident or disease may bring us to an untimely end, but no amount of care or coddling will enable us to live much beyond the span which Nature has allotted us. Progress in medical science has increased the average longevity of human beings by several years, but this has been accomplished largely through reducing mortality in infancy and childhood, and to a less extent in adult and middle age. The expectation of life in ages over eighty has changed very little. Even in the most healthful of all possible worlds there would probably be few centenarians. As Young has shown in his volume On Centenarians most of the reported cases of people, such as Thomas Parr, who are alleged to have lived much over a hundred years are of very dubious validity.

The general death rate in Europe and America has been declining for the past few hundred years, but the most rapid decline has occurred during the nineteenth and twentieth centuries. This is due to the more widespread diffusion of education, and the progress made in curing diseases and preventing their spread. The course of the decline for several countries is shown in the following table:

Table i 5.-Death Rates of Selected Countries, I84I-I932

\begin{tabular}{|c|c|c|c|c|c|c|c|c|c|c|c|}
\hline & $\begin{array}{c}184 \mathrm{I}- \\
\mathrm{I} 85^{\circ}\end{array}$ & $\begin{array}{l}\text { I } 851- \\
\text { I } 860\end{array}$ & $\begin{array}{c}\text { I 86I- } \\
\text { I } 870\end{array}$ & $\begin{array}{l}\text { I } 87 \text { I- } \\
\text { I } 880\end{array}$ & $\begin{array}{l}1881- \\
1890\end{array}$ & $\begin{array}{l}\text { I 89I- } \\
\text { I } 900\end{array}$ & $\begin{array}{l}1901- \\
1910\end{array}$ & $\begin{array}{l}\text { I9I I- } \\
\text { I9I3 }\end{array}$ & $\begin{array}{l}1921- \\
1925\end{array}$ & $\begin{array}{l}1926- \\
\text { I928 }\end{array}$ & 1932 \\
\hline England. & 22.4 & 22.2 & 22.5 & 21.4 & I9. I & 18.2 & 15.3 & 13.8 & 12.2 & II 9 & 12.0 \\
\hline France.. & $23 \cdot 3$ & 23.9 & 23.6 & $23 \cdot 7$ & 22.1 & 21.5 & 19.4 & 18.3 & 17.2 & 16.9 & 15.8 \\
\hline Germany. . & 26.8 & 26.4 & 26.9 & 27.2 & $25 \cdot \mathrm{I}$ & 22.2 & 18.7 & 16.0 & $13 \cdot 3$ & 11.8 & 10.8 \\
\hline Sweden... & 20.6 & 21.7 & 20.2 & 18.3 & 16.9 & 16.4 & 14.9 & I 3.9 & 12.1 & 12.2 & II . 6 \\
\hline Massachusetts & & 18.2 & 19.4 & 19.8 & 19.6 & I 8.9 & I6.6 & 15.2 & 12.5 & 12.0 & II. 5 \\
\hline
\end{tabular}

A part of this decline is spurious since it results to some extent from the changing age composition of the population 


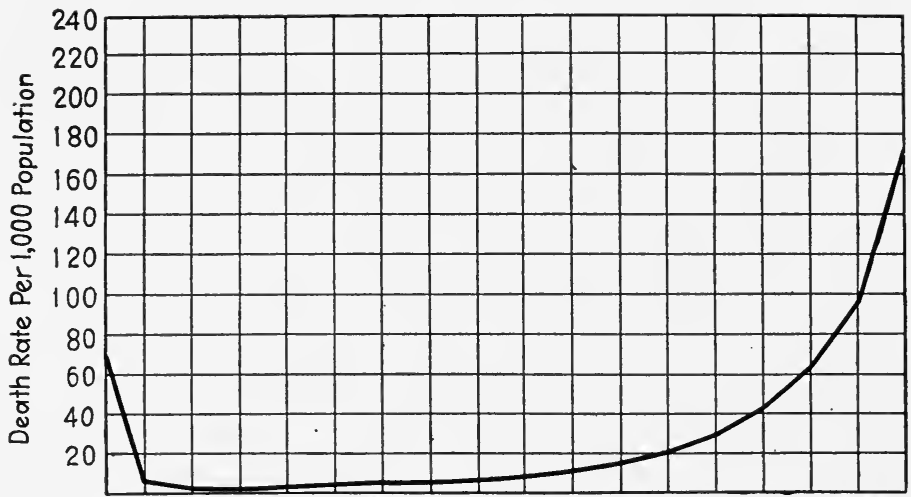

Under11-4 5-9 10-74 15-19 20-24 25-29 30-34 35-3940-4445-49 5054 55-5960646569 70-74 75-79 80 and Years of Age

Fig. 65.-Curve illustrating death rates at different ages in the United States in $193^{\circ}$. (After Willcox.)

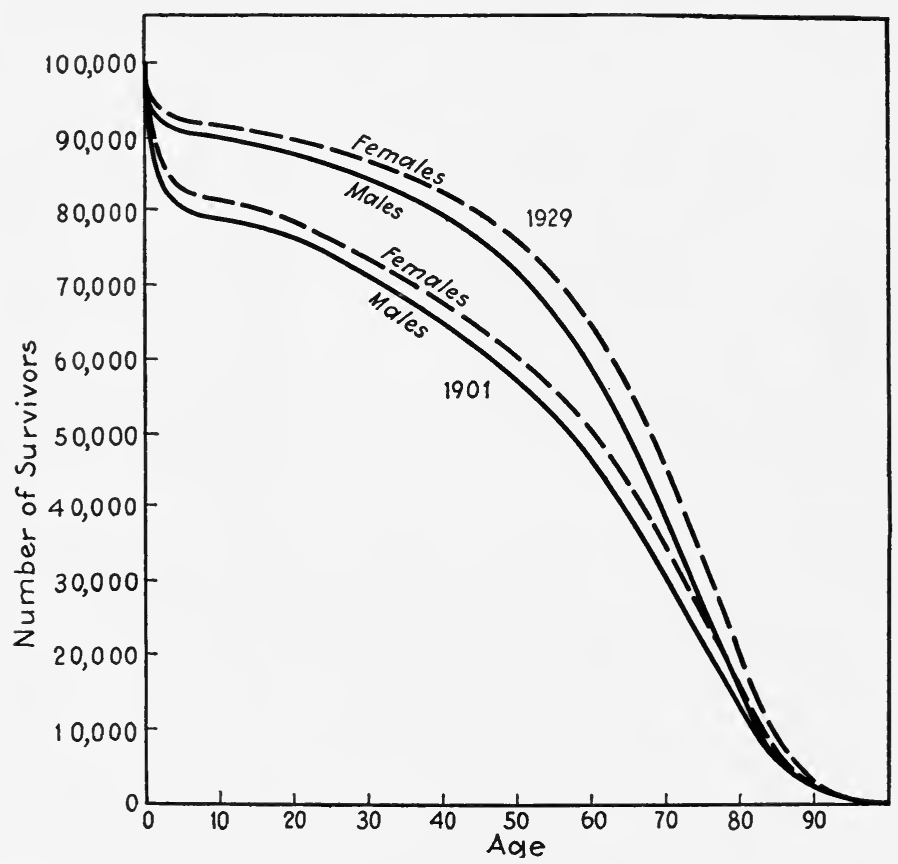

Fig. 66.-Number of survivors of each age and sex out of every 100,000 born in the original death registration states in 1901 and 1929 . (After Wiehl.) 


\section{HUMAN GENETICS AND ITS SOCIAL IMPORT}

arising from the decline of the birth rate. As the proportion of infants and young children becomes reduced, the death rate in a population will also be reduced. Thus for several years past, the age composition of the population of Europe and the United States has been relatively favorable for a low death rate. The populations of most countries are growing older, i.e., the average age of people is increasing. In the course of time this will cause the death rate to become higher again when it leads to a relatively large proportion

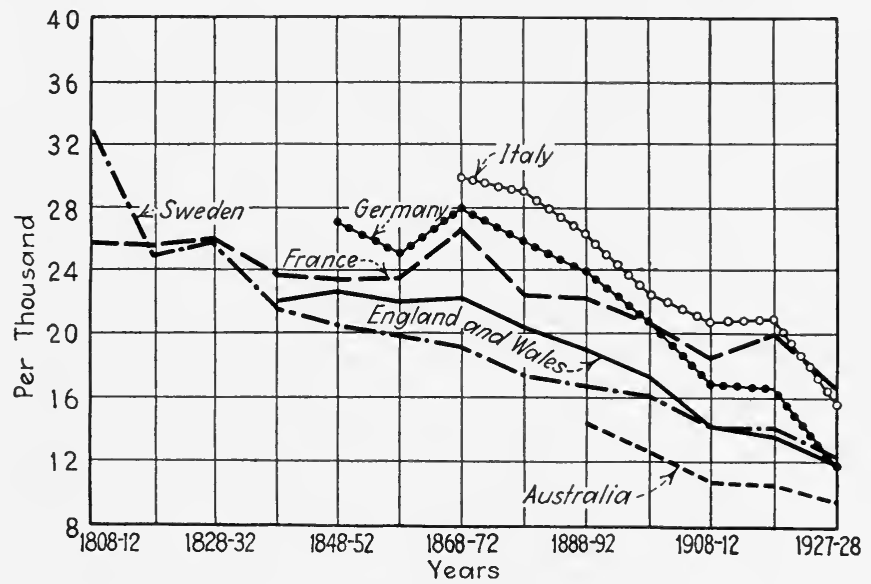

Fig. 67.-Death rates of European countries, 1808 to 1928. (From Thompson.)

of people in the older age groups; but so long as the proportion of people in the relatively healthy periods of life is increasing, the death rate will fall.

One large factor in the real decline of the death rate is the reduction of infant mortality. For several reasons it is desirable to have a special index of the infant death rate. Instead of being expressed in terms of thousands of the population under one year of age, a matter which is difficult to determine with much accuracy, it is customary to express it as the number of infants dying in a year out of every $\mathrm{I}, \infty 00$ infants born during that year. Formerly infant mor- 
tality was high everywhere, and in many regions it still continues to be high. During the past few years it has fallen rapidly in several countries. There has thus been a partial compensation for the fall of the birth rate, although the number of children kept from dying is far less than the number who have been kept from being born.

Table i6.-Infant Mortality Rates per Hundred Births, i871-I930

\begin{tabular}{|c|c|c|c|c|c|c|c|c|c|c|}
\hline & $\begin{array}{c}1871- \\
1880\end{array}$ & $\begin{array}{l}\text { I } 88 \text { I- } \\
\text { I } 890\end{array}$ & $\begin{array}{l}1891- \\
1900\end{array}$ & $\begin{array}{l}1901- \\
1905\end{array}$ & $\begin{array}{l}1906- \\
1910\end{array}$ & $\begin{array}{l}1911- \\
\text { I9I } 5\end{array}$ & $\begin{array}{l}1916- \\
1920\end{array}$ & $\begin{array}{l}1921- \\
1925\end{array}$ & $\begin{array}{l}19^{26}- \\
1928\end{array}$ & 1930 \\
\hline England and Wales. & 14.9 & 14.2 & $15 \cdot 3$ & 13.8 & I I .7 & II.O & 9.0 & 7.6 & 6.8 & 6.0 \\
\hline Germany........ & $23 \cdot 4$ & 22.5 & $21 \cdot 7$ & 19.9 & 17.4 & 16.0 & I $4 \cdot 5$ & I 2.2 & 9.6 & 8.4 \\
\hline France......... & 17.2 & 16.7 & 16.4 & 13.9 & I2.6 & I 2.4 & I2.I & $9 \cdot 4$ & 9.0 & 7.8 \\
\hline Norway........ & 10.5 & 9.8 & $9 \cdot 7$ & 8.I & 7.0 & 6.6 & 6.2 & $5 \cdot 2$ & 5.0 & 4.6 \\
\hline Italy.... & 20.9 & $19 \cdot 5$ & 17.6 & 16.7 & 15.2 & $I_{4} \cdot 0$ & 15.0 & 12.5 & I 2.2 & 10.5 \\
\hline
\end{tabular}

The reduction of infant mortality has often been assigned as one of the causes of the declining birth rate. Where family

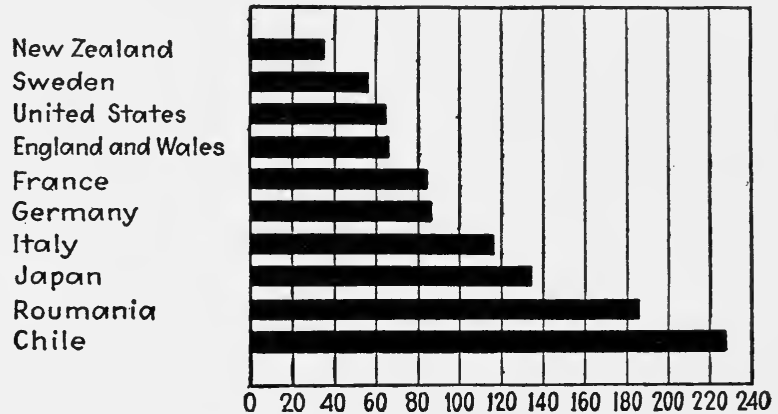

FIG. 68.-Infant mortality rates (per 1,000 births) in different countries. (From Thompson.)

size is standardized, the death of a child often causes the parents to desire another child in its stead. Hence the more frequently children die the more frequently they will tend to be born. On the other hand, it is often urged, especially by the Neo-Malthusians, that a high birth rate tends to cause a high infant mortality. Where there are many children who have to be supported on limited resources, conditions 


\section{HUMAN GENETICS AND ITS SOCIAL IMPORT}

in the home are apt to be less favorable for the health of the children. With fewer children and a better standard of living infant mortality is naturally reduced.

There is a general tendency for high birth rates and high infant mortality rates to go together, although there are occasional exceptions to this rule. This fact does not prove that the high birth rate is the cause of the high infant mortality or vice versa. Both may derive from a common cause in the ignorance or improvidence of the parents. Under primitive conditions of life many children are born and many die. When people become more enlightened they regulate the birth supply, and at the same time they prevent the early death of more of their offspring. As in so many other situations in human biology, the causal relations involved do not all appear on the surface. A low infant mortality may be partly caused by a low birth rate. Also a low birth rate may be partly caused by a low infant mortality. But, perhaps to a greater extent, both are caused by other factors.

Obviously death rates in infants and adults alike are greatly affected by the surroundings in which people live. Climate, food, diseases, parasites, and other hazards to health, produce striking differences in the mortality rates of different regions of the earth. The first attempt to construct a canal across the Isthmus of Panama occasioned such an appalling mortality among the workers that it had to be abandoned. Through overcoming many environmental causes of death, biological discoveries have now made it safe to live in many regions formerly almost uninhabitable. Further discoveries will doubtless add to the average duration of human life, but how much longer can human beings hope to live?

In 1930 the average expectation of life of white males in the United States was 59.0 years and that of white females, 62.62 years. In 1901 it was 48.23 for white males, and 
$5^{1.08}$ for white females. Over ten years had been added to the average duration of life in three decades. In 1789 the average expectation of life was estimated roughly to be about thirty-five years. There have been some attempts to project estimates of longevity into the future. According

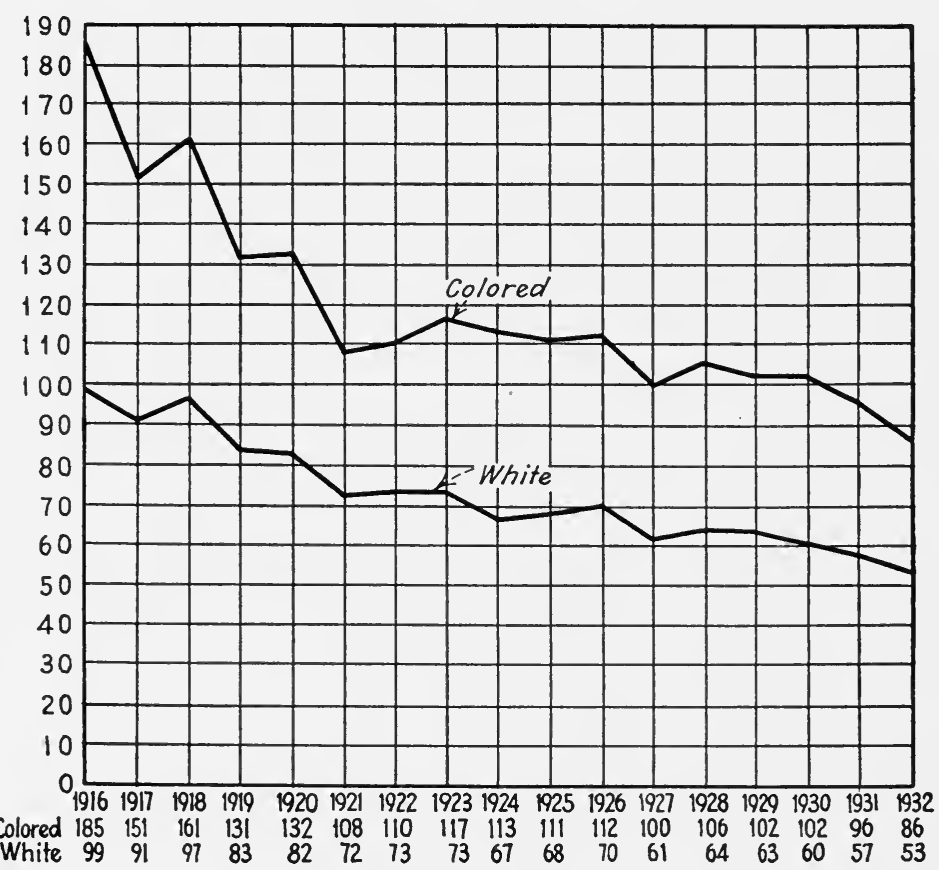

FIG. 69.- The decline of the infant mortality rate (per 1,000 children born) in the white and colored population of the birth registration area of the United States, I9I6 to $193^{2}$. The curves for the whites and the colored have similar ups and downs. The rise in 1918 was due to the epidemic of influenza.

to the estimates of Whelpton, American whites of the year I 980 may expect to have an average longevity of seventythree years-which is a little better than the Scriptural allotment of three score years and ten-but they cannot look forward to much increase after that. When this happy time arrives people in their eighties will be standing on every street corner, and it might be found that there were too many of them for the good of the country. 


\section{HUMAN GENETICS AND ITS SOCIAL IMPOR'T}

One factor which affects mortality, as was pointed out by the able vital statistician Dr. William Farr, is density of population. The more thickly people are massed together the higher will be their death rate. According to Dr. Farr, the mortality of a population increases as the sixth root of its density, but this rather venturesome attempt at mathematical precision known as Farr's law has not been found to be of general validity, especially under modern conditions of life. Doubtless density of population is one con-

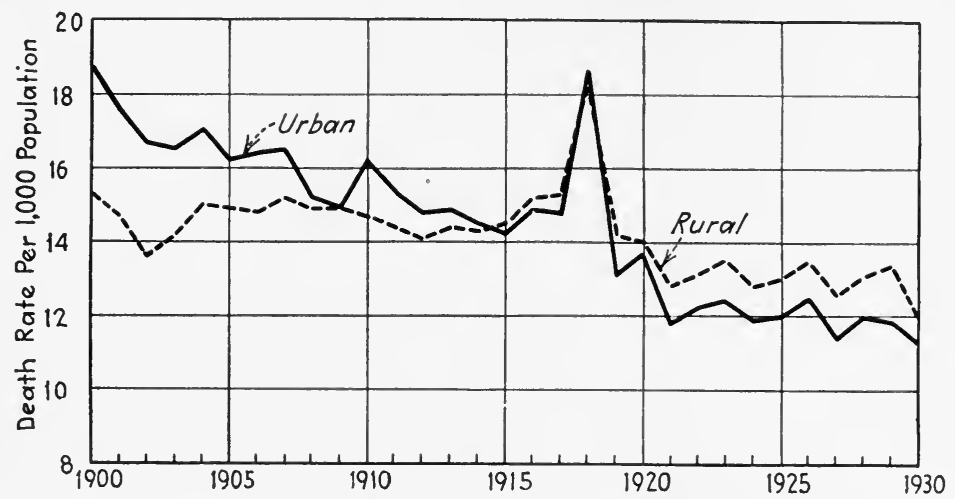

Fig. 70.-Death rates in urban and rural areas in the Registration States of 1900, I900 to 1930. (After Willcox.)

tributing cause of the relatively high mortality of people living in cities. Where people are crowded together in the slums, poverty, disease, and unfavorable surroundings conspire to take a heavy toll of life, but in some cities many people may live in limited areas in which conditions are relatively wholesome. It is not surprising, therefore, that the relation between density of population and the death rate should be found to be of the most general character and subject to many exceptions.

For a long period the death rates of urban dwellers have been higher than those of their rural compatriots, so much so that the populations of most cities, and especially large 
cities, have been incapable of maintaining themselves without being replenished from the surrounding country. In general it is the rural areas which supply the material for population growth, and since larger and larger proportions of the population of most civilized countries are coming to live in cities, the demographical effects of urbanization constitute a factor of increasing importance in the biological changes occurring in the human species.

For several decades over one-half of the population of several European countries (England, Holland, Belgium, Germany) have lived in cities. The population of the United States has long been mainly rural (it was over 95 per cent rural in r800), but the census of 1920 showed that the population was over 5 I.4 per cent urban and 48.6 per cent rural. In I930 it was 56.2 per cent urban and 43.8 per cent rural. This enormous growth of cities, involving as it does a decrease in the birth rate and an increase in the death rate of ever larger proportions of the population, has acted as a check upon the more highly civilized peoples of the earth.

The trend of urban mortality like that of mortality in general has been downward, especially in recent years as is shown in the following table.

Table 17.-Death Rates in Large Cities, i 866-I930

\begin{tabular}{|c|c|c|c|c|}
\hline Date & Berlin & Paris & London & Vienna \\
\hline I 866-I 870 & $3 I \cdot 9$ & 26.8 & 24.4 & 34.2 \\
\hline I $871-1875$ & 32.7 & & & \\
\hline I $876-$ I 880 & $29 \cdot 3$ & $25 \cdot 5$ & 22.5 & 32.9 \\
\hline I $88 \mathrm{I}-\mathrm{I} 885$ & 26.5 & $24 \cdot 4$ & 21.0 & 28.5 \\
\hline I 886-I 890 & 22.5 & 23.0 & 20.0 & $25 \cdot 5$ \\
\hline I $89 \mathrm{I}-1895$ & 20.5 & 21.2 & 19.8 & $24 . I$ \\
\hline $1896-1900$ & I 8.1 & I9. I & 18.5 & 21.1 \\
\hline $1901-1905$ & 17.0 & 17.9 & I6. I & I9. I \\
\hline I906-1910 & 15.2 & 17.8 & 14.7 & 17.1 \\
\hline $1921-1926$ & I 2.1 & 16.4 & 12.2 & I 5.1 \\
\hline $193^{\circ}$ & II . 2 & I $3 \cdot 3$ & I I .9 & 13.4 \\
\hline
\end{tabular}




\section{$25^{\circ}$ HUMAN GENETICS AND ITS SOCIAL IMPORT}

In some regions the urban death rate is lower than in the surrounding rural area. This is frequently due to the favorable age composition of the city population. Dorn has shown that in Ohio, for instance, the crude death rates of the native whites were higher for both sexes in the rural than in the urban population, but that if the rates are adjusted for age composition the urban rates are higher than the rural.

Table i 8.-Death Rates per Thousand Native Population of Ohio, Urban AND RuRAL

\begin{tabular}{|c|c|c|c|c|}
\hline \multirow{2}{*}{ Rate } & \multicolumn{2}{|c|}{ Male } & \multicolumn{2}{|c|}{ Female } \\
\hline & Rural & Urban & Rural & Urban \\
\hline Crude. . & II .O & $9 \cdot 9$ & 10.5 & 8.8 \\
\hline 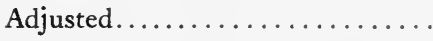 & 8.7 & 10.0 & 8.3 & 8.6 \\
\hline
\end{tabular}

In some respects city life frequently offers conditions more favorable for low mortality than those of the country. Nowadays, the water supply of most large cities is free from the contaminations that were formerly responsible for the spread of cholera, typhoid fever, and other diseases. Inspection of the milk supply has aided in reducing mortality in infancy and childhood, and the efficient health administration and superior medical services of many cities have gone far toward overcoming the natural handicaps of urban life. Also, in several regions the education and standard of living are higher in cities than in the surrounding country. For these and other reasons the relation between the mortality of city and country is subject to much variation. In general, death rates have fallen more rapidly in urban than in rural areas. This is in part due to the simple fact that there was more room for improvement. Even infant mortality rates, which have usually been much higher in urban areas, have now fallen so much the more rapidly in cities that they are sometimes lower than in the surrounding country. 
In the United States Registration Area for Births the urban and rural rates of infant mortality as shown in the following table have declined at unequal rates. Up to I 928 the urban rates have been the higher. For the last few years, however, the urban rates have been lower than the rural.

Table 19.-Mortality Rates of White Infants in the Birth Registration Area of the United States, I919-1932

\begin{tabular}{c|c|c|c|c|c|c|c|c|c|c|c|c|c|c}
\hline & 1919 & 1920 & 1921 & 1922 & 1923 & 1924 & 1925 & 1926 & 1927 & 1928 & 1929 & 1930 & 1931 & 1932 \\
\hline $\begin{array}{c}\text { Urban } \\
\text { whites.. }\end{array}$ & 86 & 87 & 75 & 77 & 75 & 69 & 69 & 70 & 61 & 65 & 62 & 58 & 56 & 53 \\
$\begin{array}{c}\text { Rural } \\
\text { whites.. }\end{array}$ & 80 & 76 & 70 & 69 & 72 & 65 & 67 & 69 & 60 & 63 & 64 & 61 & 57 & 54 \\
\hline
\end{tabular}

So far as their death rates are concerned, there seems to be no reason why cities should continue to be the centers of extinction that they have been in the past. It is chiefly their very low birth rate that makes cities such potent destroyers of humanity. And there is little reason to conclude that urban birth rates will increase.

\section{Suggested Readings}

Newsholme ('23), chaps. 17, 19-21. Thompson ('35), chap. I2. Thompson and Whelpton ('33), chap. 7. Willcox, ('33).

\section{Questions}

I. What information concerning longevity is furnished by a life table? Look up your own expectation of life and your chance of dying within a year. Compare these findings with your vital prospects when you reach age sixty.

2. Look up the death rate and infant mortality rate of your own city or town. How do births compare with deaths?

3. How would an increase of the birth rate affect the general death rate? Explain. Would it necessarily involve an increase in the infant mortality rate as the latter is commonly measured?

4. How would an increase of mortality after age forty-five affect the marriage rate? the birth rate? the insanity rate? 


\section{HUMAN GENETICS AND ITS SOCIAL IMPORT}

5. How would the death rate of a city be affected by the presence of an insane asylum, a college, army barracks, a large cannery?

6. Why do you think that mortality is higher in unmarried men than in the married? Does this fact prove that marriage increases longevity?

7. City $A$ has a death rate of eleven per thousand and city $B$ has a death rate of fourteen per thousand. Is city $A$ necessarily the more wholesome place in which to live? Explain.

8. If a city has a death rate of nine per thousand what inference would you draw as to its age composition? If there were no migrants into or out of the city how would the death rate be likely to change in subsequent years? Explain.

9. Compare for both sexes in the United States life tables for I9I0, I919-1920, and I930 the expectation of life at ages ten, fifty, and eightyfive. What do the results indicate as to the increase in the span of life as compared with the increase in the average duration of life?

Io. Is it likely that a community can maintain indefinitely a death rate of ten per thousand inhabitants? If so, what would be the average duration of life?

II. Look up in any United States life table the death rates of the two sexes, whites and Negroes, native-born and foreign-born inhabitants at ages one, five, ten, thirty, fifty, seventy, eighty-five, and over. How would you interpret these findings? 


\section{CHAPTER XIX}

\section{THE SELECTIVE ACTION OF MORTALITY}

$A$ CCORDING to the theory of natural selection proA pounded by Mr. Darwin, the differential death rate plays a role of major importance in the evolution of life. Darwin conceived of all organisms as engaged in a struggle for existence, not merely against the forces of their environment, but with competitors of their own and other species. "Owing to this struggle for life," he says, "any variation, however slight, and from whatever cause proceeding, if it be in any degree profitable to an individual of any species, in its infinitely complex relations to other organic beings and to external nature, will tend to the preservation of that individual, and will generally be inherited by its offspring. The offspring, also, will thus have a better chance of surviving, for, of the many individuals of any species which are periodically born, but a small number can survive. I have called this principle, by which each slight variation, if useful, is preserved, by the term of Natural Selection, in order to mark its relation to man's power of selection. We have seen that man by selection can certainly produce great results and can adapt organic beings to his own uses, through the accumulation of slight but useful variations, given to him by the hand of Nature. But Natural Selection, as we shall hereafter see, is a power incessantly ready for action, and is as immeasurably superior to man's feeble efforts, as the works of Nature are to those of Art."

Natural selection is regarded as an agency always acting upon organisms, continually preserving adaptive variations 


\section{HUMAN GENETICS AND ITS SOCIAL IMPORT}

that may arise and thus ever tending to bring about a more adequate adjustment of the organism to its environment. Darwin believed also in the Lamarckian theory of the transmission of acquired characters, but he thought that this factor played a distinctly subordinate role in the process of organic evolution.

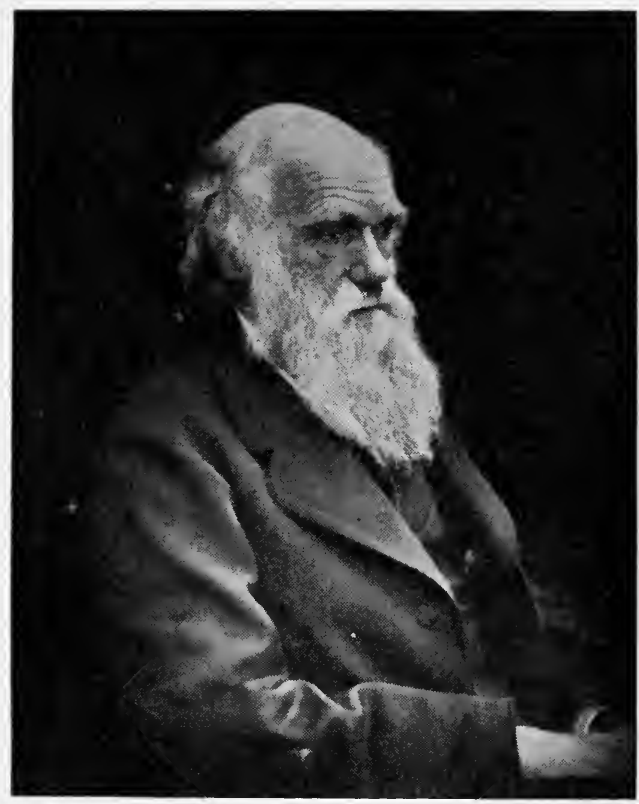

Fig. 71.-Charles Darwin. (From photograph by Major Leonard Darwin.)

Whether Darwin's theory of natural selection affords a satisfactory explanation of evolution is a much argued question which we shall not attempt to discuss. That organisms are preserved or eliminated as a result of differences in hereditary constitution has been abundantly demonstrated by observation and experiment. There can be no doubt, therefore, that natural selection is a force which is in active operation in the organic world whether we consider that it gives a sufficient explanation of evolution or not. 
Before discussing the operation of natural selection in the human species it should be pointed out that in a broad and general sense natural selection embraces both the differential death rate and the differential birth rate. The former aspect of the selective process was emphasized most by Mr. Darwin, although it is clear that he conceived of natural selection as including the latter as well. Since we have considered the differential birth rate we shall confine our attention to the selective action of mortality or, as it is sometimes called, lethal selection, as contrasted with reproductive selection, which is based on differences in reproductive rates.

One way in which the action of natural selection is shown in man is through the inheritance of longevity. It is a wellknown fact that there are long-lived as well as short-lived families. The matter has been subjected to a mathematical investigation by Professor Karl Pearson who studied the longevity of families listed in Foster's Peerage, Burke's Landed Gentry, and, in connection with Miss Beeton, in the Society of Friends. Correlation coefficients for length of life were worked out for fathers and sons, fathers and daughters, mothers and sons, mothers and daughters, and for siblings. The correlations found are given in the following table:

TAble 20.-Correlations for Length of Life

Correlation

Father and son over 24, peerage............. 0. I I $5 \pm 0.021$

Father and son over 19, landed gentry.......... 0.142 \pm 0.021

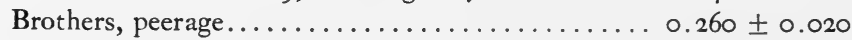

Brother and adult son, friends................ $0.135 \pm 0.021$

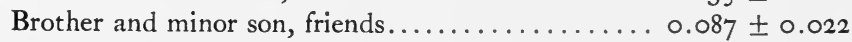

Mother and adult daughter.................. 0.149 \pm 0.020

Mother and minor daughter................ 0.1 $38 \pm 0.024$

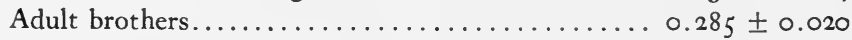

Older and younger brothers................... $0.229 \pm 0.019$

Adult sisters...................... 0.332 \pm 0.019

Most of these correlations, as well as those found by Pearl and others, are relatively low as compared with correlations 


\section{HUMAN GENETICS AND ITS SOCIAL IMPORT}

for most physical and mental characteristics. This is due in part to the large element of fortuitous mortality involved. It is of interest that the correlations between siblings are higher than those between parents and offspring, whereas if the resemblances were due merely to heredity we should expect about the same degree of correlation. One is hardly justified in considering all of the correlations between parents and offspring for length of life as due to heredity, since a part of it may result from similarities of environment to which both parents and offspring are exposed. There is also a danger of obtaining a spurious correlation for longevity if we base our conclusions on data extending over a period of time in which the average duration of life has changed. In studying correlations for length of life in royal families I have found that if we take only those individuals living in a given half century, the correlations for length of life are considerably less than those obtained by including records covering two or more centuries. Doubtless both heredity and environment conspire to cause longevity to run in families, but it is difficult to obtain separate measures of the extent of their influence.

According to Pearson, not only length of life, but general health is inherited. There is also, as the studies of Pearson, A. G. Bell, and others have shown, a tendency for longevity to be correlated with fertility. Long-lived, healthy, and fertile stocks, therefore, tend, other things equal, to prevail over and supplant short-lived, unhealthy, and infertile stocks. Of course, accidents, maleficent microbes, and other causes of untimely death interfere with the truly selective action of mortality. But, on the whole, natural selection is ever acting to weed out the maladapted and to cause the better endowed types to replenish the earth.

The operation of natural selection in man is also shown by the fact that there are many kinds of hereditary defects 
and disorders which lead to the elimination of their possessors. Hemophilia, which is inherited as a recessive, sexlinked character often results in death through bleeding caused by a failure of the blood to clot. Natural selection, therefore, tends to eliminate strains that inherit this defect. Similarly, Huntington's chorea tends to be eliminated although many individuals marry and transmit the defect before their fatal malady overtakes them. Amaurotic family idiocy probably depends upon a recessive gene and since the trait is uniformly fatal very early in life, the strains which carry it will tend, although very slowly, to die out. Several other hereditary maladies are known which result in death at various periods of life. However, there are others that are responsible for deaths in early stages of embryonic development as there are in several species of animals.

A great deal of selective elimination results from hereditary diatheses to germ diseases and other types of disorder. In so far as tuberculosis depends upon a hereditary proclivity to that extent natural selection tends to eliminate the more susceptible elements of the race. How far the increasing immunity of peoples long exposed to tuberculosis is due to this cause is, however, difficult to ascertain. There are hereditary diatheses to diabetes, heart disease, several types of malignant growths, many nervous disorders, and other defects and diseases, all of which reduce the chances of survival. It may be said that natural selection is always busy at the task of eliminating from the human race its many kinds of hereditary defects and diseases. One might suppose that since natural selection has been engaged in this enterprise for many hundreds of thousands of years, to say nothing of the millions of years before man became man, the human race, by this time, would be freed of its burdens of hereditary deficiency. Doubtless it would have 


\section{HUMAN GENETICS AND ITS SOCIAL IMPORT}

been were it not for the appearance of new mutations, most of which from the standpoint of survival, are either indifferent or disadvantageous. In this respect man is in the same situation as other organisms in which genetical study has shown that new mutations, mostly unfavorable, are being continually produced. Most of these new experiments are speedily eliminated by natural selection. Only occasionally does a mutation arise which better fits the organism to survive in the struggle for existence. Hence, if all kinds of variations were to survive and propagate, the disadvantageous ones would gradually accumulate, and the species would slump backward to a lower level of efficiency. This tendency to slump which results from the cessation of natural selection was called by Weismann panmixia. It represents a factor which is always working in the interests of degeneration, and to which the occurrence of rudimentary organs and degenerate types of organisms was in part attributed.

It is commonly held that natural selection in man has largely been set aside through our arts of saving lives. In primitive men, whose survival often depends upon strength, acuity of sense and mental alertness, such variations as split hands, club feet, hereditary deafness and blindness, or other disabling infirmities that are now perpetuated through several generations, would be quickly eliminated. Perhaps one reason why we are so heavily burdened by defective heredity is because unfavorable variations have been allowed to accumulate, which normally would have led to extinction. Some writers are seriously concerned over the prospect that our fostering the weak will cause the race to become more and more decrepit as time goes on. There is no danger, however, that we shall ever be able to eliminate entirely the operation of natural selection, and the extent to which we have been able to reduce its action has commonly been exaggerated. To a large extent mortality has been re- 
duced through the removal of causes of death whose action is mainly fortuitous. Some causes of death like hemophilia are highly selective; some, such as lightning are mainly nonselective; and others are selective in various degrees. The elimination of causes of death which are about as apt to kill one person as another can do no racial damage, but if the advances of medical science result in the increase of constitutionally inferior people, there is no escape from the conclusion that they are responsible for a real danger to the race. Probably this danger can be obviated by the proper eugenic measures-but this matter belongs to a later stage of our discussion.

In endeavoring to ascertain how civilization has influenced the action of natural selection it is important to consider the relation of mortality to levels of intelligence. The rate death of low-grade imbeciles and idiots is relatively high. Of the 625 idiots and imbeciles concerning whom he had mortality records Dr. Barr found that "the largest number of deaths occurred between ten and twenty years; but comparatively few passed the twenty-fifth year and exceptional cases occurred from thirty to forty years." According to Clark and Stowell the lowest grades of mental defectives in the hospitals and schools of New York City have a death rate four times as high as the feeble-minded in general, and among the latter the death rate is about twice as high as among normal children. Since the lowest types of mental defectives are more or less pathological products, their high death rate is to be expected. Dayton finds that in Massachusetts the expectation of life of mental defectives at two years of age increases as the degree of defect decreases. For idiots it is twenty-one years for females and twenty years for males. For imbeciles it is thirty-eight for females and twenty-nine for males and for morons it is forty-six for females and fifty-two for males, whereas in the general 


\section{HUMAN GENETICS AND ITS SOCIAL IMPORT}

population of the state it is fifty-nine for females and fifty-six for males.

Evidently natural selection is tending to get rid of mental defectives. To a certain extent it is doing the same with the insane. The death rate of inmates of asylums for the insane is much higher than that of the general population of corresponding ages. Epilepsy is not infrequently associated with early death. And there are other nervous disorders in which heredity plays a large part which either shorten life or prevent marriage and the procreation of children.

Passing to the normal ranges of intelligence levels, we find that in general there is an inverse relation between intelligence and mortality. In the professional classes and in such groups as English men of science studied by Galton, American men of science studied by Cattell, and the people whose names get into Who's Who, the death rate is low. This is due not merely to the fact that as a rule a person has to be twenty-five years old or more to be included in these groups, but it is due to the greater life expectancy, age for age, as compared with that of the general population. It is safe to assume that these classes, with occasional exceptions of course, are characterized by a degree of intelligence somewhat above that of the rank and file of human beings. So far as death is a respecter of persons, we may conclude that in the highest and in the lowest mental levels it is working in the way that the eugenist might desire. How deaths are distributed in relation to levels of intelligence within the broad belt of humanity lying between these extremes, we have to judge mainly on the basis of data on the mortality of the several occupational groups. There are certain sources of error involved in the interpretation of such data. Some dangerous trades take a heavy toll of their workers quite irrespective of the intelligence required in these pursuits. Agricultural laborers do not 
rate very brilliantly on the basis of intelligence tests applied to their offspring, but their death rate is low owing to the wholesome nature of their work in a rural environment. Besides, people often change their occupations. Early in life they may be casual laborers, and especially in the United States they may rise from humble tasks such as splitting rails to much higher employments. A comparison of the crude death rates of different occupational groups is especially apt to be misleading, because the age composition of the groups is subject to great variation. There may be many persons below twenty among unskilled laborers, but practically none among doctors or lawyers.

We may take as typical of the general character of occupational mortality the results of a study made by Dr. T. H. C. Stevenson on the data supplied by the Census of England and Wales in I92I-I923. A general sample of the findings is shown in the accompanying table:

Table 21.-Mortality Per 100,000 Occupied and Retired Males I 5-65 in England and Wales, I921-I923

\begin{tabular}{l|c|c|c|c|c|c|c}
\hline Class & I5-I9 & $20-24$ & $25-34$ & $35-44$ & $45-54$ & $55-64$ & Infant mortality \\
\cline { 2 - 6 } I & I $42^{2}$ & 237 & $26 \mathrm{I}$ & 484 & 985 & 2,247 & 38 \\
II & 205 & 307 & 376 & 589 & $\mathrm{I}, 090$ & 2,469 & 55 \\
III & 243 & 347 & 380 & 590 & $\mathrm{I}, 070$ & 2,508 & 77 \\
IV & 248 & 367 & 420 & 669 & $\mathrm{I}, \mathrm{I} 73$ & 2,482 & 89 \\
V & 299 & 408 & 498 & 880 & $\mathrm{I}, 507$ & $3,06 \mathrm{I}$ & 97 \\
\hline
\end{tabular}

It may be explained that Class I in the table includes the upper and professional classes; Class III consists of skilled artisans; Class $\mathrm{V}$ is composed of unskilled and casual laborers; and the Classes II and IV include workers of a status intermediate between those of Classes I and III, and III and V respectively. The table shows that in each group the mortality rises as we pass from the upper to the lower occupational classes. The same general tendency is 


\section{HUMAN GENETICS AND ITS SOCIAL IMPORT}

shown by the occupational mortality of other countries. In so far, therefore, as these occupational groups are characterized by different average levels of intelligence, we may say that natural selection is operating on the basis of mental endowments. It is a matter of interest and perhaps much biological significance that the infant mortality in these groups follows the same course as the mortality of the parents.

In our modern industrial world the morons and dull normals are relatively poor actuarial risks. They gravitate

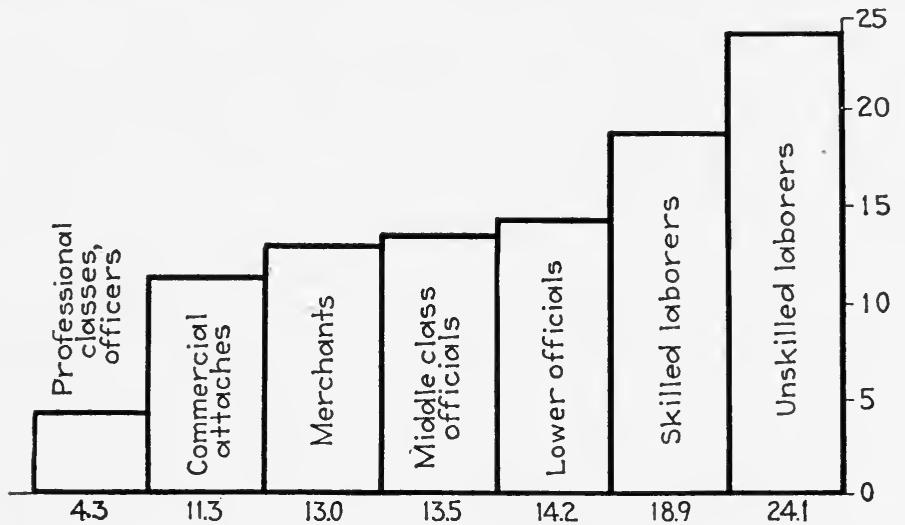

Fig. 72. - Infant mortality rates per 100 births according to occupation in Prussia, 1912I914. (Data from Rott.)

into the lower occupational groups in which the pay is poor and in which living conditions are not the best. Besides, their progeny suffer from a high infant and child mortality. So far as its lethal aspects are concerned natural selection tends to eliminate dull minds as it eliminates weak bodies. It is not unreasonable to suppose that the effect of our modern industrial development through creating a great diversity of employments and subjecting human beings to very unequal environmental influences has been to cause natural selection to act with a greater degree of discrimination on the basis of variations in intelligence. When occupations were little diversified and most people followed similar 
pursuits and lived in much the same way, the average lot of the dull-normal individual was not greatly different from that of the exceptionally intelligent. In our industrial world inequalities of station are associated with differences in mortality to a greater extent than is found in a less highly differentiated economic order. We may have partly overcome the action of natural selection in eliminating the physically weak and abnormal, but at the same time we may have made it more highly selective as regards levels of intelligence.

Natural selection is not only hard on the moron, but also on those whose peculiar emotional and temperamental traits make them social misfits. In so far as criminals, vagabonds, paupers, chronic alcoholics, and other types of human wreckage are the victims of their unfortunate heredity, it may be said that lethal selection is working to rid society of these undesirables. Their mortality is relatively high, and their children, when they have any, suffer from a high death rate. Among women prostitution tends to eliminate a class whose heredity on the average is probably of a rather inferior sort. According to a number of studies the intelligence level of prostitutes is low (most of them come from the lower occupational groups), and there is a considerable amount of mental abnormality, although how far the latter is the cause instead of the effect of their calling is uncertain. They are of ten sterilized by venereal diseases, or their children suffer from a high mortality from inherited syphilis and unfavorable surroundings. Although prostitutes come from relatively prolific stocks, it is probable that as a class they are not a self-perpetuating group-which is doubtless a fortunate circumstance from both the racial and the social point of view.

Many of the discussions on the role of natural selection in man have been concerned with the selective operations 
of infant mortality. From the evolutionary standpoint, it matters relatively little what happens to people after they have ceased to reproduce their kind. The fact that in the United States mortality after age forty-five has been actually increasing, may be unfortunate for the welfare of society and especially for the life insurance companies, but it has little significance for biological evolution. Since the large part of human mortality before the end of the reproductive period occurs in infancy and early childhood, the extent to which the death rate is selective during this period is of especial biological importance. When we consider the appalling death rate of infants in many regions and the fact that all infants require much care and protection for their successful upbringing, it is evident that many wellendowed infants must perish along with the weaklings. Moreover, many infants are exceptionally weak and puny as a result of environmental causes such as premature birth or temporary ill health of the mother. Once the perils of early life are past these infants may develop into exceptionally strong and healthy individuals. As man gradually evolved from lower forms, infants became progressively weaker and in need of more and more care. Similar developments have occurred in many kinds of birds in which the young are the most helpless and dependent of creatures kept alive only by the active ministrations of their parents. Infantile weakness of this sort is not related to any constitutional inferiority of later life and evolution in the direction of greater weakness in early life is entirely compatible with the biological advancement of the species. It would be a mistake, however, to conclude that all infantile inferiority has no evolutionary significance.

The problem of how far infant mortality is selective on the basis of genetic differences has been the subject of much controversy. The idea that any biological advantage might 
result from the selective elimination of young infants is viewed with evident alarm by many humanely disposed people. They like to feel that progress in saving the lives of helpless infants has not resulted in the accumulation of inherent weaknesses or defects, and they are led to emphasize, perhaps unduly, the nonselective character of infant mortality. Those who contend that natural selection operates in the first year of life have often been accused of advocating the abolition of the hygienic procedures which have led to the salvage of so many infant lives. It is one thing to point out that the reduction of infant mortality has its racial dangers and another thing to defend the barbarity of an infant death rate that sweeps away every third or fourth child. The question at issue can be settled by an appeal not to sentiment but to facts. If the death rate in infancy is not affected by genetic differences this period is unlike every other stage of life. Deaths in infancy may be due to fortuitous and nonselective causes to a greater extent than in subsequent years - this seems likely-but it is hardly reasonable to suppose that hereditary factors are not responsible for a part of the mortality occurring at this time. Amaurotic family idiocy, which is commonly fatal in the first year of life, is probably dependent upon recessive genes, and there are other maladies that work in a similar way.

It has been shown in a number of studies that infant mortality is relatively low in long-lived stocks. Data collected by Ploetz from royal and princely families in Germany indicate that where children were raised under as favorable conditions as the times afforded, the mortality under five years of age decreased as the age at death of either the father or the mother increased. The relations are shown in the subjoined table. Much the same relations were disclosed in families of the middle class. In studying the inheritance of longevity in New England genealogies I find that as a 


\section{HUMAN GENETICS AND ITS SOCIAL IMPORT}

Table 22:-Child Mortality as Related to the Mortality of Children in Royal and Princely Families

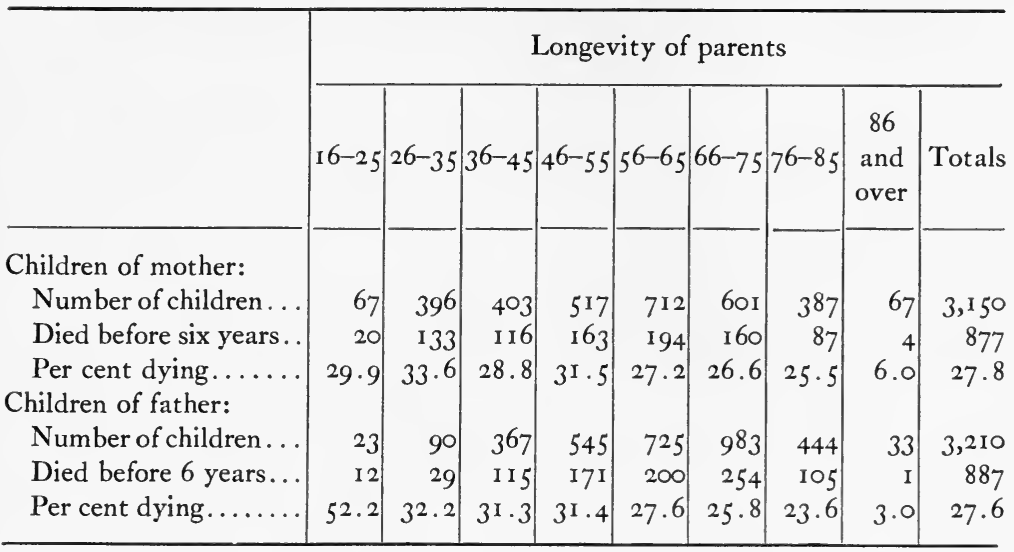

rule the infant death rate is low as the age at death of the parents increases. The relations were as follows:

Table 23.-Parental Longevity and Infant Mortality According to New England Genealogies

\begin{tabular}{|c|c|c|c|c|c|c|c|c|}
\hline & \multicolumn{8}{|c|}{ Longevity of parents } \\
\hline & $10-25$ & $25-35$ & $35-45$ & $45^{-55}$ & $55^{-65}$ & $65-75$ & $75^{-85}$ & $85^{-}$ \\
\hline Fathers: & & & & & & & & \\
\hline No. of children. & 2 & 70 & I68 & 268 & 388 & 547 & 560 & \\
\hline Number dying under 5 years. & $\mathbf{I}$ & 17 & $3^{8}$ & 66 & 77 & 96 & 110 & \\
\hline Per cent dying under 5 years. & 50.0 & 24.29 & 23.17 & 24.63 & 19.84 & $17 \cdot 55$ & 19.64 & 13.83 \\
\hline Mothers: & & & & & & & & \\
\hline No. of children. & I3 & 93 & 145 & 185 & 290 & 408 & $50 \mathrm{OI}$ & 337 \\
\hline Number dying under 5 years. & & 32 & 45 & 36 & 46 & 70 & 96 & 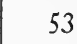 \\
\hline Per cent dying under 5 years. & 38.46 & $34 \cdot 40$ & 31.03 & 19.46 & 14.86 & 17.16 & $\mid 19.16$ & $15 \cdot 72$ \\
\hline
\end{tabular}

From the preceding data it seems likely that the genetic constitution which enables people to attain long life also helps their infants to escape the perils of the hazardous first year.

In some studies made by Pearson, Snow, and Crum an attempt has been made to demonstrate the action of natural selection in early life by showing that there is a 
negative correlation between the infant death rate and the later mortality of childhood, the inference being that when more children die in infancy the survivors are less apt to die later on. This conclusion has been attacked by a number of critics who for the most part have advanced irrelevant arguments against it and have failed to call attention to its most vulnerable point of attack, namely, the fact that several causes of infant deaths, such as the infectious diseases of children, confer an immunity upon the survivors. If, for instance, children are exposed to measles at age one and again at age five, fewer will die from measles at the latter age, not necessarily because measles removed the weaklings at age one, but because the group has been immunized and hence protected against a later attack. On account of the influence of immunity we may conclude therefore that the existence of a selective death rate is not proved by the negative correlation referred to; neither is it disproved. There is a certain presumptive evidence of a differential death rate based on variations in vitality afforded by the ratio of male to female deaths in early. life. At this time boys are more prone to die than girls - a fact for which Nature seems to have made provision in that more boys than girls are born. The sex ratio at birth is about ${ }^{0} 5$ to 106 boys to 100 girls. In deaths during the first year the ratio of boys to girls varies from nearly $\mathrm{I}_{4} \mathrm{O}$ to about IIO. The lower the mortality rate the higher is the ratio of boy deaths to girl deaths. It is a striking fact that the sex ratio at death is highest soon after birth and falls rather rapidly after the second or third month to the twelfth, and after that more slowly through the years of childhood. The sex ratio at death in the United States (1916-1923) was as follows for the several subdivisions of the first year:

$$
\begin{array}{ccccccc}
\text { Month } & 0-1 & 1-2 & 2-3 & 3-5 & 6-8 & 9^{-12} \\
& 137.2 & 138.4 & 134.1 & 130.6 & 123.4 & 116.6
\end{array}
$$


In stillbirths the sex ratio (boys to Ioo girls) is high. If we follow the course of sex mortality back into uterine life, we find that it is high in early abortions (of about the fourth month or pregnancy), and decreases until about the seventh month, after which it rises until the critical period of birth. In the U. S. Registration Area for Births the sex ratio of abortions over a period of several years were 375 for the third month, 210.6 for the fourth, I 45 for the fifth, a lower ratio for the sixth, and the lowest of all for the seventh, II8.4. After this it rises, being I2I in the eighth month and 137.9 in the ninth.

The high death rate of boy babies has been attributed to the fact that they are a little larger than girl babies and have somewhat larger heads. Hence they are more apt to suffer from the ordeal of birth, which is often a severe one for the child as well as the mother. The sex ratio of children dying from injuries at birth in the Birth Registration Area of the United States from I9I4 to I923 was I6I.6 in whites and I 55.9 in Negroes. Evidently, therefore, birth injury is one source of high male mortality in infancy, but this fact cannot explain the very high sex ratio of early abortions.

Why, therefore, do boys die so much more frequently than girls both in utero and in infancy and childhood? It has been suggested that this may be due to recessive sex-linked factors. It has also been suggested that boys are inherently weaker than girls as a result of the peculiar chromosome complex which causes them to be boys. From all causes of death (with the exception of whooping cough and, for anatomical reasons, gonococcus infection) boys die more readily than girls - a circumstance which is indicative of a greater constitutional weakness. This greater physical toughness of the female is characteristic of the human species throughout life, as may be seen by consulting any life table. There are more women than men in the older age groups, and 
their expectation of life in the eighties is higher than that of men.

During infancy there is much variation in sex mortality from different causes of death. The sex ratio is high for malformations of the heart 143.8 , intestinal obstructions I62.8, hernia 37I.2, nervous diseases 134.6 , nephritis 147.3 , and relatively low from external causes II 3 , and some epidemic diseases. That the sex ratio is high in proportion as the general infant mortality rate is low may be explained on the ground that as nonselective or partly selective causes of death are removed, the death rate which remains in spite of all efforts to reduce it is based to a relatively greater extent upon genetic differences associated with sex. It would seem probable that causes of death which are responsible for a high lethal sex ratio are also selective within each sex on the basis of inherent differences in vitality.

There is a kind of selective elimination in infancy not directly connected with toughness of constitution on the part of infants themselves, but which may be of great importance from the racial standpoint, namely, selection on the basis of parental intelligence. A young moron may be a tough and husky physical specimen, but if he is born to parents who are also morons, his chances of surviving infancy are thereby considerably reduced. Poor food, inadequate care, the wrong kind of treatment during illness, and other disadvantages resulting from the ignorance and stupidity of parents, are responsible for the deaths of many physically normal infants. Natural selection, therefore, bears heavily upon the progeny of the dull and stupid. As we pass up the scale of the various occupational groups we find a steady decrease in infant mortality as in the mortality of adult life. In a broad and general way infant mortality is correlated with parental intelligence although 


\section{HUMAN GENETICS AND ITS SOCIAL IMPORT}

this relationship is obscured by the effect of poverty and its attendant drawbacks. It has been shown in several investigations that infant mortality is strongly associated with low wages of the father. In a study of infant mortality in seven large cities of the United States the relation of the infant death rate to the earnings of the father as well as the race and nationality of the mother were found to be as indicated in the following table:

Table 24.-Infant Mortality Rates in Seven Cities According to the Color and Nativity of the Mother and the Earnings of the Father ${ }^{1}$

\begin{tabular}{|c|c|c|c|c|}
\hline \multirow{2}{*}{ Earnings } & \multicolumn{4}{|c|}{ Mortality rates } \\
\hline & Total & Native white & Foreign-born white & Colored \\
\hline Average ......... & I 10.0 & $93 \cdot 9$ & $123 \cdot 7$ & 152.3 \\
\hline Under $\$ 450 \ldots$ & 166.9 & 170.0 & 167.1 & 162.7 \\
\hline$\$ 450-\$ 549 \ldots \ldots \ldots$ & 125.6 & 121.0 & II 8.4 & 163.7 \\
\hline$\$ 55^{\circ}-\$ 649 \ldots$ & I16.6 & I 10.8 & 121.8 & 122.8 \\
\hline$\$ 650-\$ 849 \ldots \ldots \ldots$ & $107 \cdot 5$ & $99 \cdot 5$ & 119.6 & 102.7 \\
\hline$\$ 850-\$ 1,049 \ldots \ldots$ & 82.8 & 76.4 & $94 \cdot 9$ & \\
\hline$\$ 1,050-\$ 1,249 \ldots \ldots$ & 64.0 & 62.6 & 68.4 & \\
\hline$\$ 1,250$ and over.... & 59.1 & 57.6 & 60.0 & \\
\hline No earnings........ & 210.9 & $187 \cdot 5$ & 234.2 & \\
\hline
\end{tabular}

1 After Woodbury.

It is unquestionable that the striking differences in infant mortality rates here indicated are to a large extent the result of the social, economic, and educational status of the family. How far differences in intelligence levels may be responsible for differences in infant mortality within each group we do not know. Infant mortality has been found to be correlated with many things, i.e., poverty, educational status, age of parents, high birth rate, interval between births, order of birth, employment of mothers, overcrowding, and breast feeding. The problem of disentangling all the causes involved is beset with formidable difficulties. Pearson has found that there is a correlation of $0.2 \mathrm{I}$ between infant 
mortality and the use of a "baby pacifier, an india rubber tantalizer, and a bacilli collector pushed between the baby's lips, at which it sucks ineffectively and indefinitely." Do the baby pacifiers kill the babies, or are both high mortality and the use of these devices associated with the ignorance and carelessness of the mothers? The latter seems to be the more probable conclusion. To how great an extent poverty, ignorance, and various other factors involved have a real causal connection with infant mortality is capable of only a partial solution. Ashby in his volume on Infant Mortality has remarked that "The unanimous verdict of the doctors who made the observations are that neither the surroundings of the infant nor the exact character of the milk obtained were as important factors in the health of the infant as the intelligent character of the mother." Even amid squalid surroundings - perhaps even more there than elsewhere- the intelligence of parents has much to do with the survival of their offspring.

In the light of the facts set forth in the present chapter we may conclude that on the whole the action of the differential death rate is eugenic in that it eliminates both the physically weak and the mentally dull. But lethal selection alone does not determine the direction of biological evolution. This is due to the combined action of lethal selection and reproductive selection, or natural selection in the broader inclusive sense of this term. In our modern life these two factors frequently work at cross purposes. From the evidence available reproductive selection is as a rule the more effective influence. When we consider the proportions of surviving children we find that the high birth rate classes usually have the greater net fertility despite their higher mortality. The net reproductive rates for six large occupational classes in the United States for 1928 have been calculated by Lorimer and Osborn as follows: 
Table 25.-Net Reproductive Rates in Different Occupational Classes Net

Reproductive

Class

Rate

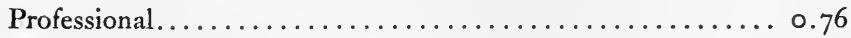

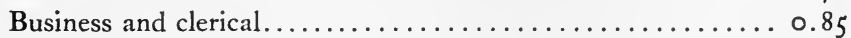

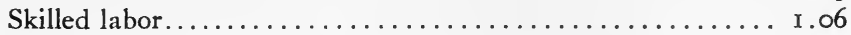

Semiskilled labor...........................

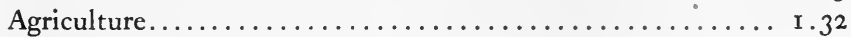

Unskilled labor....................... I 7

The two first groups are not reproducing themselves and will have to be recruited from the lower groups, thus draining them of the more intelligent hereditary strains that they still possess. This situation which is characteristic of our present industrial age, whatever it may have been in past times, may possibly be a temporary phase. In some countries, as we have seen, the recent decline of the birth rate has been more rapid in the lower social classes. A similar trend is observed in infant and child mortality. When infant mortality is as low as 40 per thousand it is difficult even were it racially desirable to reduce it much further, but where it is I 50 to 200 it readily yields to improved conditions of nurture. Not improbably the death rate as well as the birth rate may come to be more nearly on the same level in the proletariat and in the upper and professional classes. We may hope that the net results of the selective processes will be on a more eugenic basis than they seem to be at the present time.

\section{Suggested Readings}

Darwin ('96), chap. 4. Dublin ('17). Holmes ('30), ('31), ('33), chap. '5. Pearson, ('12).

\section{Questions}

I. Why, according to Darwin, does natural selection cause varieties to diverge until they become distinct species?

2. In what way does the theory of natural selection explain the evolution of adaptive structures? 
3. Why did Darwin concede that natural selection cannot account for a character in a species primarily of value only to some other species?

4. Does a high death rate necessarily imply that natural selection is in active operation? Explain.

5. Mention six selective and six mainly nonselective causes of death in man in addition to those referred to in the text.

6. In what ways does natural selection act differently from the way it acted in former times?

7. Can voluntary selection ever compensate entirely for the action of natural selection?

8. What characteristics have been explained on the theory that natural selection acts upon groups instead of upon individuals per se?

9. How do you think the race would be affected by a reduction of infant mortality to twenty per thousand births?

Io. What reasons are there for thinking that natural selection is active during embryonic development?

II. Mention ten hereditary defects of the eyes. Do you think that such defects are liable to accumulate in the future?

12. What ground do you think there is for concluding that man faces "a bald and toothless future"?

13. What do you think will be the racial effect of obstetrical procedures to facilitate childbirth?

14. Discuss the possible atrophy of the mammary glands in the future of the human species.

15. From the standpoint of biological fitness is it better for the human species to live under conditions which entail a high death rate? 


\section{CHAPTER XX}

\section{THE BIOLOGICAL EFFECTS OF WAR}

T CONSIDERING the influence of war on the biological 1 evolution of the human species it is important to remember that by nature man is a fighting animal. Nature has equipped many kinds of animals with fighting instincts and special weapons for offense and defense which are convincing testimonies as to the importance of conflict in the struggle for life. The horns of deer, sheep, and cattle, the protective mane of the male lion, and the powerful tusks of the wild boar are all structures whose development has doubtless been determined by their service in encounters the issue of which is often a matter of life or death. Anger with its concomitant the fighting instinct is a common attribute of higher types of animal life. Typically anger is aroused by some sort of interference. The dog resents the removal of his bone, and the large male sea lion drives away all rivals for any of the females that he has managed to appropriate. Children usually get on well together until one child takes a plaything belonging to another or otherwise interferes with some privilege or the free exercise of some activity, and then there is trouble. We may consider the fighting instinct as one expression of the will to live. Its biological usefulness is obvious, especially in a world in which no rights are respected that cannot be defended.

One of the most conspicuous features of the fighting instinct in animals is that it frequently takes the form of group pugnacity. This is especially true with animals that live in social groups. Most insects are quite devoid of combative propensities. The struggles of the predatory forms 
to overcome their prey cannot properly be described as fighting; they are merely incidental to the regular business of procuring food. Most insects cannot be provoked to resist any kind of interference, but among the social insects it is a different matter. Highly social groups have been independently evolved in the insects among the ants, the bees, and the wasps, and also among the remotely related termites. And all these insects exhibit a high degree of pugnacity. Nevertheless, an ant, bee, wasp, or termite rarely resents an interference with its activities as an individual, but its ire is at once aroused by any danger threatening the habitation of its group. Stir up a hornet's nest, an anthill, a bumblebee's nest, or a group of termites, and the hostile reaction will be prompt and decided. These insects are ready to fight to the death in the defense of their group; and in many species of both ants and termites a special caste of warriors with large heads and strong jaws has been evolved in the service of defense. The pugnacity of these creatures has nothing of self seeking; it is almost entirely altruistic. Nature has endowed them with instincts which lead them to risk their lives that their fellows may be protected. Individuals are pawns in the game and are sacrificed with little hesitation in order that the game may be won.

In the insects, group pugnacity has been evolved pari passu with the development of social life. It goes along with instincts for mutual aid upon which all highly developed social life depends. The same principle is also manifested among the less highly socialized birds and mammals. A timid bird may fly in the face of an enemy which threatens her young.

For the poor wren,

The most diminutive of birds, will fight,

Her young ones in the nest, against the owl. 


\section{HUMAN GENETICS AND ITS SOCIAL IMPORT}

Among chimpanzees which commonly run in troops there is a strong tendency for individuals to keep closely associated, and an attack made upon any member brings the whole crowd upon the offender. Among chimpanzees kept in captivity a newcomer is regarded with suspicion if not positively attacked, but after a time the stranger becomes adopted as one of the group. The rule with these social animals is helpfulness toward members within the group and hostility to outsiders. To a certain extent this is the rule also among tribes of primitive men. In fact, a certain degree of clannishness is characteristic of all human beings, however cultivated.

According to the Darwinian theory the social sympathy and instincts for mutual aid in social animals have developed because of their utility in promoting social solidarity and effective cooperation. In the struggle for existence group is pitted against group, and those having the instincts leading to mutual support tend to supplant other groups with less cooperation. A society in which each member promotes the welfare of his fellows is more apt to survive than one in which each individual is concerned exclusively with his own welfare. As Karl Pearson has remarked in his excellent lecture on National Life from the Standpoint of Science, "The safety of a gregarious animal-and man is essentially such-depends upon the intensity with which the social instinct has been developed. The stability of a race depends entirely on the extent to which the social feelings have got a real hold on it. . . . No tribe of men will work together unless the tribal interest dominates the personal and individual at all points where they come into conflict. The struggle among primitive men of tribe against tribe evolved the social instinct. The tribe with the greater social feeling survived; we have to thank the struggle for existence for first making man gregarious, and then intensifying, 
stage by stage, the social feeling. Such is the scientific account of the origin of our social instincts; and if you come to analyze it, such is the origin of what we term morality; morality is only the developed form of tribal habit, the custom of acting in a certain way toward our fellows, upon which the very safety of the tribe originally depended."

From the Darwinian standpoint, which is so clearly expressed in the passage quoted, both man's fighting instinct and the higher qualities which lead him to give unselfish service to his fellows are alike produced by natural selection for the sake of their survival value in the struggle for existence. If this be the true interpretation of why we are both kindly and pugnacious, conflict must have played an important part in making man a social animal. Even the gentlest and most unselfish of the original impulses of human nature may be viewed as products of the intergroup struggle for survival. To express the matter in somewhat cynical terms we may say that it pays to be kindly to one's fellows in order to be more effective against one's enemies.

During the long period of human evolution before the beginnings of recorded history, conflicts must have occurred between relatively small groups. This is true of primitive peoples today. Only in advanced stages of civilization is it possible for peoples to become welded together into large organized societies. When this is done the working of group selection suffers marked changes. And with the progress of invention there came to be great changes also in the methods of waging war. In the hand to hand encounters of primitive men the strongest and most resourceful contestant was the most apt to survive and hand on his traits. Victory often meant the extermination or enslavement of at least the men of the conquered group, although the Children of Israel were rather more thorough in killing the men, women, and children of their unfortunate enemies. Wars of extermination 


\section{HUMAN GENETICS AND ITS SOCIAL IMPORT}

have been carried on by some tribes of North American Indians, the Dyaks, and other primitive peoples, but even among most savages the victors usually spare the women and children and often many of the men of the conquered tribe. Not infrequently there is a mingling of the blood of the victors and the vanquished, but notwithstanding this fact primitive warfare is, on the whole, probably eugenic in its effects. With the advance of civilization the biological effects of war become subject to many changes. As was pointed out by Herbert Spencer "Though, during barbarism and the earlier stages of civilization, war has the effect of exterminating the weaker societies, and of weeding out the weaker members of the stronger societies, and thus in both ways furthering the development of those valuable powers, bodily and mental, which war brings into play, yet during the later stages of civilization, the second of these actions is reversed. . . But when the industrial development has become such that only some of the adult males are drafted into the army, the tendency is to pick out and expose to slaughter the best-grown and healthiest; leaving behind the physically-inferior to propagate the race."

The dysgenic effects of modern warfare to which Spencer calls attention have been strongly emphasized by many recent writers. Dr. D. S. Jordan devoted much of the energy of his later years to setting forth the destructive biological influence of war. In collaboration with $\mathrm{H}$. E. Jordan he made an inductive investigation of the actual effects of the Civil War on the population of a selected area of Virginia. It was shown that the leading men of the community were the first to enlist, and that a large percentage of them ( 20 to 40 per cent) died before the close of the war; that war took the physically fit, the unfit remaining behind; that the volunteers, who were on the whole superior as soldiers to the conscripts, suffered more severely, and that, 
on the whole, "the men of highest character and quality bore largely the brunt of the war and lost more heavily than their inferiors." University men both north and south volunteered in large numbers early in the war, and their death rate in battle was higher than that of the rank and file of the soldiers. During this war the South especially

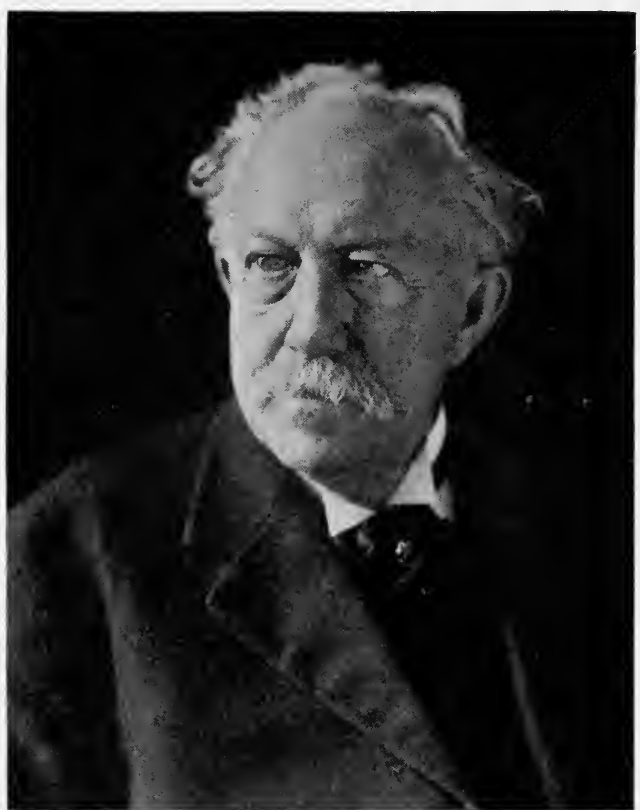

FIG. 73.-David Starr Jordan. (From Eugenics.)

suffered grievously from the loss of its best man power, a loss which will continue to be felt for many years.

The removal of those fit for military service tends to give the unfit, the deserters - there are many of these in all warsand those who contrive through lack of courage or patriotism to avoid participation in the struggle, a greater opportunity to marry and beget offspring. According to Lenz, modern warfare, instead of causing men to be more courageous and patriotic has the reverse effect, since those possessing these 


\section{HUMAN GENETICS AND ITS SOCIAL IMPORT}

socially desirable qualities are eliminated, while those lacking them tend to be spared to hand on their qualities to their descendants. Hence war would make a people less warlike. This might not be a matter of regret if it did not involve the sacrifice of other qualities, which the race can ill afford to lose.

As to the effect of selective elimination on those taking part in battle the data for different wars are not in entire agreement. The losses among officers are commonly greater than among the common soldiers, except in the case of generals and other superior officers who are usually at a safe distance behind the firing line. The German storm troops, who were an exceptionally efficient group, sustained much higher losses than the average soldiers during the World War. The losses in the flying corps were terrific, and also among the picked men who manned the submarines. Of those who entered service from the universities and gymnasia of Germany, it is estimated that not more than half were living at the close of the war. The flower of the youth from the universities of France, England, Belgium, and Austria suffered an exceptionally high death rate; and in the United States, which suffered relatively little, the losses among the college youth, to judge from the studies of Hunt in the case of Harvard University, were higher than in the rank and file of the soldiers.

The losses sustained by armies are by no means restricted to the battle field. Most wars have been accompanied by epidemics of various kinds which have often proved to be more fatal than the attacks of the enemy. In the SpanishAmerican War several times as many soldiers died of diseases as were killed by the Spaniards. During the Crimean War four times as many soldiers died of diseases as were killed at the front. With the advance of medical science the proportions dying of disease in recent years have been 
greatly reduced. Despite the length of the World War only about a tenth of the losses of the Germans were due to disease, although the armies of some of the other nations suffered more severely.

In considering the selective effects of mortality due to war one must reckon with the deaths occasioned in the civil population. During war there is commonly an increased death rate and especially an increase of infant mortality among the noncombatants. As a result of the Thirty Years' War the population of Germany was reduced to one-half or one-third of its former numbers. Owing to circumstances growing out of the World War the civil population of Russia lost many millions. Usually it is the civil population of the defeated countries which suffers most severely. Doubtless the greatest handicaps are in all cases borne by the economically poorer classes, but beyond this fact there is little to indicate how the selective action of mortality works out in the general population. If a severe ordeal leaves a stronger race of survivors, war may have a eugenic influence on the civilians which may counteract, to a certain extent, its dysgenic effects upon the combatants. We know little concerning the way in which selection works in the nation at large, and it may work very differently in different cases.

This is probably true also of the mortality on the firing line. Losses occurring in armies composed of more patriotic volunteers may be highly dysgenic, whereas those sustained by an army raised by a draft which spares the more intelligent and competent persons engaged in industry may not be more dysgenic than the death rate in normal times. Whether our army in the World War had as high an average intelligence as the male population in general is very doubtful. The extent to which military selection is dysgenic, and even whether it is dysgenic at all, depends on circumstances which vary from war to war. The dysgenic effects of war 


\section{HUMAN GENETICS AND ITS SOCIAL IMPORT}

have often been greatly exaggerated. That, as has been contended, Greece, Rome, and other ancient countries fell as a result of degeneration caused by the loss of their best men who were killed in battle is a very questionable generalization. Whatever decadence these people suffered was probably due much more to a differential birth rate than to a differential death rate. Probably war often works dysgenically, but it is by no means the greatest of dysgenic ills.

Thus far we have considered only the effect of war on the genetic constitution of the individuals composing the group. But war is not merely a struggle between individuals, for tribe contends against tribe and nation against nation. Organized groups function as units in the struggle for existence, and, granting that war takes the best within each group, if it leads to the replacement of an inferior by a superior people, it may result in biological advancement. During the course of history there has been a successive replacement of one kind of people by another. In so far as war leads to the survival of better endowed stocks it may be said to be an important factor in the biological evolution of the human species.

That war constitutes an indispensable agent of progressive evolution is the favorite thesis of those who defend it upon biological grounds. Conspicuous among these is General Bernhardi whose book Germany and the Next War attracted widespread attention just before the conflict of I9I4. "In the extrasocial stuggle, in war," says General Bernhardi, "that nation will conquer which can throw into the scale the greatest physical, mental, moral, material, and political power, and is therefore the best able to defend itself. War will furnish such a nation with favorable vital conditions, enlarged possibilities of expansion and widened influence, and thus promote the progress of mankind; for it is clear 
that those intellectual and moral factors which insure superiority in war are also those which render possible a general progressive development. . . . Without war inferior or decaying races would easily choke the growth of healthy budding elements, and a universal decadence would follow. ... Might is at once the supreme right, and the dispute as to what is right is decided by the arbitrament of war. War gives a biologically just decision, since its decisions rest on the very nature of things."

However the process of group selection may have operated in past times, modern warfare rarely leads to the replacement of one people by another. A defeated nation may suffer loss of territory and have to pay indemnities, but its population may go on increasing and may grow more rapidly than that of its victorious rival. Consider the wars that have occurred in the modern history of Europe. The inhabitants of no country have been exterminated as a result of defeat. From the biological standpoint a country may be said to be victorious whose population is caused to multiply the more rapidly as the result of a war. It frequently happens that the military victory is won by one country and the biological victory by another. Moreover, most European nations are composed of a variety of ethnic elements so that only to a minor degree do conflicts occur between natural groups. A conflict along lines of real biological cleavage would throw each country in Europe into a many-sided civil war. From the biological standpoint all the bloody wars by which Europe has been devastated have resulted only in a futile waste of life. Certain countries may gain power, wealth, and prestige as a result of a successful war, and these are the chief things for which they fight; but when these ends have been attained terms of peace are agreed upon which usually have no relation to or concern with the biological stuggle for existence. As a 


\section{HUMAN GENETICS AND ITS SOCIAL IMPORT}

matter of fact victory or defeat has usually had little relation to population growth.

Where humanitarian considerations have prevented the extermination of the vanquished or their forcible expulsion from the conquered territory, group selection of the kind postulated by the proponents of the biological value of war simply does not work out. An exceptional case is presented when primitive people of a sparsely settled country are conquered by a more highly civilized nation. It cannot be doubted that the Anglo-Saxons were enabled to increase greatly in numbers owing to a series of successful conflicts with the aboriginal inhabitants of North America, Australia, and New Zealand who resisted their advance. The result was that a primitive people was largely supplanted by those of a higher culture. To a less extent wars of this type have led to the increase of the Spanish, Portuguese, and French in the foreign lands which they acquired through conquest. But conditions favorable for this sort of racial replacement are growing distinctly less as civilization advances. During the past four centuries primitive peoples in many regions have given way before the advance of the whites. Most parts of the tropics are unsuitable for permanent colonization by members of the white race. The policy of the whites has been to control and exploit these regions, and so long as such efforts are successful the tropics may contribute to the support of an increased white population. In almost all parts of the world in which whites control dependencies having a large number of colored inhabitants, the originally hostile relations have been succeeded by a reign of peace. In the exploring and settling period the relations of whites and aborigines were mainly antagonistic. As a result, native peoples in many parts decreased in numbers. Many tribes of American Indians have become greatly reduced in numbers or completely eliminated. The Australian aborigines have 
been decreasing in numbers as the white population has grown and extended its domain. The Tasmanians are completely gone. Throughout Polynesia and Melanesia an extensive depopulation has been going on which has threatened with extinction the inhabitants of many islands. In large parts of Africa that have been raided to supply the slave trade there has been a marked decrease of the native population. The whites have proved to be a deadly scourge to many of their less enlightened competitors. As a result of the advent of the whites, native peoples have suffered not only from war but from the forcible recruiting of labor, alcoholism, the demoralization of established customs, and from many diseases which at times have decimated their ranks. During its expansion the white race has wrought fearful havoc upon many primitive peoples. In this period natural selection in the form of racial replacement was in very active operation and resulted in rapid and extensive changes in the population of the globe.

But the succeeding period of peaceful relations presents us with a very different spectacle. Under the settled conditions of white control the natives, instead of being driven to the wall, have come to receive many advantages through their association with a more highly developed race. Medical science and sanitation have contributed greatly to reduce the death rate. Internecine wars have been largely abolished. Humane and considerate treatment has replaced the original relations of hostility; but more important than all these is the fact that through the development of natural resources the whites have made it possible to support a much larger indigenous population than was formerly able to subsist. Native peoples are coming to work into advantageous industrial relations with their white employers, and the whites have come to recognize in native labor a valuable financial asset which it is profitable to conserve and increase. 
Natives have gained much from the economic opportunities, medical services, educational advantages, and stable administration afforded by the more enlightened peoples in control. The status of primitive peoples has gradually changed from that of enemies that must be overcome to members of an industrial society enjoying in common with the whites the benefits of peaceful cooperation.

It is doubtless owing in large part to this change of status that the primitive peoples of various parts of the world have come to show a positive increase after a period of declining numbers. The widespread depopulation going on in the islands of the Pacific inhabited by Polynesians and Melanesians has given place in most islands to a surplus of births over deaths. The Maoris of New Zealand, who have frequently been referred to as a disappearing race, have been increasing for several years, and the I93I Yearbook of New Zealand states that they have been increasing more rapidly than the Europeans. The native inhabitants of Java have increased over tenfold under the administration of the Dutch, and as a result of British rule the population of thickly inhabited India has increased over 50 per cent. There has been a marked increase of the Negro inhabitants of the parts of South Africa under British control, and it is not unlikely that the recent increase in the rate of growth of the Negro population of the United States is the result of more advantageous relations with the dominant whites.

In recent years the effects of white control in various parts of the globe have been to increase the growth rate of native peoples. This affords a larger supply of cheap labor which the employing classes have found it financially profitable to conserve. Purely humanitarian motives have, of course, played a part in leading the whites to promote the welfare of their more primitive neighbors, but the motive of economic interest has probably played the more effective role. 
It is important to bear in mind that whatever may be the immediate economic advantages resulting from the association of whites and more primitive peoples, the different races are none the less biological rivals. The whites have promoted the increase of their colored brethren in many parts of the world. And throughout the world there has been a growing spirit of resistance to white domination. Have the whites been raising up potential enemies that some day they will no longer be able to control? The interracial struggle for existence has entered upon a new phase which differs sharply from the previous period of active strife, in that conditions favor the more rapid increase of the subject peoples. This increase will involve a greater consumption of food, leaving less available for export. As the world fills up with people, those countries that depend on other regions for their food supply and for materials for their manufacturing industries may find their position growing increasingly difficult. Several of these countries now have a birth rate that is considerably below what is necessary for maintaining a stationary population, and are faced with the possibility of an actual decrease in numbers in the not distant future. And there is still room in the world and means of support for many additional millions, and according to some estimates several additional billions of people. From what quarters will the additional population come? The days when the white race sent forth its millions of colonists have passed. Some of the chief sources of emigrants of the last century have dried up, and others are approaching the same condition. The future growth of world population seems destined to come largely from the prolific colored races that are yet little influenced by birth control and whose people are habituated to a low standard of living. Looming up ominously in the future is the prospect of industrial competition by the colored 
races, which may deprive the whites of many advantages that they owe to their superior technical skill and equipment. As the colored inhabitants of white dependencies increase in numbers, education, and economic efficiency, the hold of the whites upon their dominions will become more feeble, and the whites may eventually be forced to relinquish their control over many territories that are now important sources of revenue. Some of the nations of southern and eastern Europe may be able to send forth colonists for several years, but the prospect of much further expansion of the white race in foreign territories does not appear to be bright:

In the meantime the nations of Europe are torn by rivalries that lead them to impose heavy taxes on their peoples to support preparations for impending wars and to adopt economic policies in relation to neighboring countries to the disadvantage of all parties concerned. The whites are their own worst enemies. There can be no question that the devastating effects of the World War and the discords which followed it have proved to be a severe handicap to the whites in the interracial struggle for power and dominion. Grievously as some of the European nations have suffered from this conflict, they are making vigorous efforts to prepare for another one, which can result only in untold injury to all participants.

From the biological viewpoint at least all this is worse than folly. Group loyalty is a valuable asset in the struggle for existence. But there is a danger that minor group loyalties may obscure the larger loyalty to race, just as excessive and antisocial individualism may tend to destroy the minor group.

\section{Suggested Readings}

Bernhardi ('I4). Holmes ('2I), chap. 9, ('32 b). Jordan ('o7), ('15), Jordan and Jordan ('14). Nicolai ('18). Pearson ('05). Cox ('22), chap. 3 . 


\section{Questions}

I. Compare the effects of primitive warfare with those of modern warfare from the standpoint of biological evolution.

2. Among modern European nations is rapidity of population growth associated with frequency of victory in war?

3. In modern times, do the people of smaller nations commonly suffer much disadvantage as a result of their weakness?

4. How do you think that war affects the choice of mates on the part of women?

5. What is the trend of the birth rate during and after wars?

6. What is a common effect of endemic diseases upon the successful invaders of a country?

7. Does the fact that war involves a great sacrifice of life indicate that it has a dysgenic influence?

8. Does acceptance of the Darwinian theory of natural selection logically involve the justification of war on biological grounds?

9. Are wars justifiable on biological grounds which lead to the expansion of such peoples as the Anglo-Saxons?

ro. What practices of victorious peoples tend to nullify the presumed biological advantages of a successful war?

II. Did any European country gain any biological advantage as a result of the great war?

12. In the interest of evolutionary advancement should more highly developed peoples endeavor, peaceably or otherwise, to supplant their less favored rivals?

I3. If you wished to evaluate the net biological results of a war, what statistical data would you find advantageous to possess?

I4. How does war affect the mixture of different racial stocks? Consider in this respect the history of England and of Rome. 


\section{CHAPTER XXI}

\section{THE EFFECTS OF POPULATION GROWTH}

7 HE growth of populations creates many problems, 1 biological, political, economic, and social, of which only a few of the more pressing can be considered here. Widespread interest in and appreciation of population problems was greatly stimulated by the celebrated Essay on Population by the Reverend T. R. Malthus, the first edition of which was published in 1798 . There had been discussions on population before this time by Raleigh, Bacon, Franklin, Hume, Condorcet, Wallace, Townsend, and others, to say nothing of the writers of antiquity, but it was Malthus's Essay which first brought the subject prominently into the limelight. The Essay owed its origin to a series of arguments between young Malthus and his father, who was much impressed with the views of Condorcet and Godwin on the perfectability of mankind. Godwin, like Rousseau and other doctrinaire theorizers of the eighteenth century, held that most of the evils from which mankind suffers arise from the unequal distribution of power and wealth. In his Political Fustice he contended that "if human beings would do away with force and laws and live according to reason there would be no crimes, no administration of justice, and no government. Besides this, there will be neither disease, anguish, melancholy, nor resentment. Every man will seek with ineffable ardor the good of all."

This sublime confidence that the millennium could be ushered in, almost at once, young Malthus was unable to share, and it was largely in order to show the futility of Godwin's grandiose scheme and others of similar ilk that 
he was led to develop the objection that, even granting an era of peace, plenty, and happiness would follow from the proposed reforms, people would soon increase rapidly in numbers until the old scourges of poverty, disease, and hardship would be upon them again. Population, as Malthus pointed out, tends to increase at a geometrical rate. Since the number who can live at any given time or place is necessarily limited by the materials required to sustain life, a population comes to increase more rapidly than its means of support. When numbers become too great they are kept down by various checks, chief among which are war, pestilence, and famine. According to Malthus, the poverty and misery of great masses of mankind are not solely the results of the iniquitous institutions imposed upon them by unscrupulous rulers, as Godwin maintained, but they result largely from the natural impulses of human beings to procreate their kind. In a word, populations have a natural tendency to grow until conditions become so bad that further increase is automatically checked. This, as Malthus admits, "tends to subject the lower classes of society to distress and to prevent any great permanent amelioration of their condition."

It cannot be said that any of the fundamental tenets of Malthus's Essay was original. Similar ideas had been clearly though rather briefly expressed by previous writers. Malthus's achievement lay in supporting his theses by a thorough inductive investigation of conditions prevailing in different parts of the earth both past and present, as it was Darwin's great achievement to furnish a strong inductive argument for evolution and the operation of natural selection. The Essay attracted widespread attention and elicited many replies. Like Darwin, Malthus soon became "the best abused man of the age." The fact that his doctrine seemed to hold out nothing but a gloomy prospect of con- 


\section{HUMAN GENETICS AND ITS SOCIAL IMPORT}

tinued misery for the majority of mankind naturally contributed to its unpopularity. Radical reformers attacked it because it spoiled their pretty utopian schemes for remodeling society. Theologians fulminated against it because, in attributing human misery to causes intrinsic in the nature of things, it seemed like an indictment of the ways of Providence. Southey sneered at it as implying that "God makes men and women faster than $\mathrm{He}$ can feed them." A nice sort of a world this which Malthus accused the Creator of making! For a reverend gentleman such impiety was inexcusable, and especially for one who, after preaching the evils of overpopulation, should have the effrontery to marry and beget children.

Amid this chorus of vituperation there were several judicious criticisms which caused Malthus to modify certain features of his views. The Essay passed through six editions in the lifetime of its author, growing in the meantime from a modest pamphlet to a book, which led eventually to its issue in two or three volumes. Despite its unwelcome conclusions Malthus's Essay was prompted by a humanitarian spirit. It advocated what seemed to the author the one way out of the miseries which human beings bring upon themselves by following their urge to reproduce their kind. This was "moral restraint," leading to the postponement of marriage or the abstention from begetting children in marriage until adequate means were assured for raising a family. If people have the foresight and self-control required for the proper regulation of numbers in relation to means of subsistence there would be plenty for all. Labor, like other commodities, would command a price commensurate with the demands for it. Human beings, unlike the lower animals, have it in their power to keep down their members to the point at which they will not press unduly upon the means of subsistence. Population with its inherent tendency to 
increase at a geometrical rate must be checked somehow. If mankind does not employ the preventive checks, Nature will take the matter in hand and keep down the surplus by her none too gentle methods. Obviously the former method is much to be preferred.

The doctrines of Malthus have played an important part in the subsequent development of political and economic theories, and they have continued to be a fruitful subject of controversy down to the present time. One of their most important influences was in suggesting to both A. R. Wallace and Charles Darwin the idea of natural selection, and this fact has greatly enhanced their indirect influence in the social sciences. Much criticism has been wasted in wrangling over matters which are not essential to the fundamental thesis that population tends to outrun its means of support and hence brings about its own checks. A good deal of the opposition to Malthus arose from the circumstance that soon after the publication of the Essay there was a period of unparalleled production of wealth which arose from the application of science to industry. Inventions of laborsaving machinery, the employment of steam power and later electricity, the improvements of production in agriculture, the immense growth of trade, and the rapid utilization of the natural resources of many lands led to an enormous increase in the means of subsistence for the nations under Western civilization. The natural consequence of this was an extraordinarily rapid increase in numbers. During the nineteenth century the population of Europe, according to the estimates of Willcox, increased from $187,693, \infty 00$ to $406,577,000$, and that of England and Wales from $10,600,000$ to $32,435,000$ and that of the United States from 6,000,000 to $76,938,000$. Similar increases occurred in other parts of the world settled by the white race and also to a less extent among several of the colored races. 


\section{HUMAN GENETICS AND ITS SOCIAL IMPORT}

One contributory cause of this rapid increase was the reduction of the death rate owing to the advances of medical science and the general spread of enlightenment during this period, but unquestionably the more fundamental cause was the great increase of the means of subsistence which resulted from industrial development. In no era of recorded history has there been so extensive an increase in population, and probably there will never be again. The nineteenth century stands out as the one period in the development of mankind which has seen the most rapid increase in numbers.

The great increase in the means of subsistence which led to this unprecedented population growth caused many to conclude that the doctrines of Malthus had been refuted by the course of events. But Malthusianism continued to be attacked on several other grounds. Militarists who wanted more soldiers, capitalists who wanted more cheap labor, and ecclesiastics who wanted more souls to add to the adherents of their faith, united in their denunciations. Even the socialists, who one would think would be favorably disposed toward Malthus's teachings, were largely either offish or hostile. Karl Marx referred to Malthus only in terms of contempt as an apologist for the iniquities of the employing classes, but he appears not to have thought out any consistent population theory of his own.

Malthus's teachings have, as a rule, met with a more favorable reception from the orthodox economists, most of whom adopt his views with more or less modification. Recently there has been a tendency toward a "return to Malthus." It is becoming apparent that the increase of the means for supporting population growth cannot continue to go on at the pace characteristic of the last century. The basic limiting factor in population growth is food, and the possibilities of food production on this planet are strictly 
limited. Several countries in Europe do not, and some of them probably cannot, produce enough food for their own support. A good deal of Asia outside of Siberia is not very far from the same situation. As to the United States, East has estimated that "with our present standards of farming, the country will support only 166,000,000,' and we have about $130,000,000$ now. Of course, with intensive cultivation production may be increased, but if our numbers continue to increase as in the past it will not be long before no amount of cultivation would yield enough to insure us against the possibility of going hungry during the lean years. Foodexporting countries, as our own has been, will soon require all their food for their own people. The world is rapidly filling up. It would be unprofitable to discuss just how many people it is capable of supporting, but whether the saturation point will be reached in fifty or one hundred, or even two hundred years does not invalidate the conclusion that we are drawing distinctly nearer the time when the food resources of the earth will no longer suffice for further increase in numbers. Civilization also requires many other things besides food. It is hazardous to set limits to the achievements of invention in providing materials and sources of energy for the needs of industrial life, but in the matter of food man is limited to the productive power of the land with such minor supplements as can be fished out of the sea; and these resources are by no means so bountiful as is often imagined. There is a possibility that the chemists may fabricate synthetic foods, but the probability that they will ever discover a means of production that will be more effective and economical than the green plant is very remote.

There have been many speculations as to the future growth of population. Some of the forecasts are wild and fantastic, but those which are based upon a careful study 


\section{HUMAN GENETICS AND I'TS SOCIAL IMPOR'T}

of the facts are much less widely divergent. Besides the forecasts of future growth based on the possibilities of food supply there are those which depend upon a study of the course of population increase in the past. These rest upon the supposition that the growth of populations follows a fairly definite law which can be expressed in a mathematical formula. According to Pearl and Reed the typical course of population growth, like the growth of an individual organism, may be represented graphically by an S-shaped logistic curve. This means that populations at first grow

FIG. 74.- S-shaped logistic curve illustrating population growth according to the conception of Pearl and Reed.

slowly, then increase at an accelerated rate, and finally slow down as they approach their maximum size. Pearl has endeavored to show that the actual course of population growth in several countries fits such a curve with a fair degree of accuracy.

These curves approach an upper limit represented by a horizontal line that typifies the maximum population obtainable. From the equation of the curve one can calculate by extrapolation what the maximum population of a country will be, provided of course, that the grow th continues to follow the same formula as in the past. Pearl concedes that changed conditions may and actually do change the course of population growth as predicted by the formula. 
Such a change as the transition from a pastoral to an agricultural life or a marked change in industrial development, may alter very materially the course of population increase. As the eminent statistician. Sir G. H. Knibbs, remarks, "any attempt to prognosticate the future numbers of Man, by extrapolating the curve of his growth in the past must fail, not merely because we have no exact numerical record of his past, but also because the factors which determine his numbers are numerous, and are liable to momentous changes." Knibbs contends that "human populations certainly do not conform to the law of growth which the logistic curve expresses, excepting accidentally and for a limited period."

Whatever may be the precise curve or curves which most closely describe the course of human increase, it is evident that they must be of a form which indicates an approach with increasing slowness to an upper limit. The period of increasing returns, so far as means for supporting population are concerned, which appeared in several countries of northern and western Europe and the United States during the nineteenth century, has now been succeeded by a period of decreasing returns. Rates of population growth in these countries, as measured by the excess of births over deaths, are diminishing. For several countries the net rates of increase in the present century, omitting the upset period of the war, are indicated in Table 26.

The net increase in the United States before I 860 was over 30 per thousand, and over 25 for the rest of the century. Since I9I0, when it was estimated by Thompson and Whelpton to be 18.3 per thousand, it has fallen to 9.8 in 1930; 7.1 in 1931, and 6.4 in 1932. In the countries of southern and eastern Europe and in Japan net rates of increase continue high, although in several of them the birth rate has fallen. They are now in much the same 


\section{HUMAN GENETICS AND ITS SOCIAL IMPORT}

situation as the nations of northern and western Europe were fifty years ago, and they may have a similar history.

Table 26.-Net Rates of Population Increase in Several Countries during the Twentieth Century

\begin{tabular}{|c|c|c|c|c|c|c|}
\hline & $1898-1902$ & $1908-1912$ & $1918-1922$ & $1927-1928$ & 1930 & 1933 \\
\hline Austria. & 12.0 & 10.7 & 3.1 & 3.0 & $3 \cdot 3$ & I. I \\
\hline 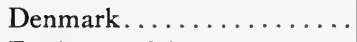 & I3.7 & I4. I & II $\cdot 3$ & 8.3 & $7 \cdot 9$ & 6.7 \\
\hline England, Wales.... & $\mathrm{I} 1.4$ & II.O & $7 \cdot 2$ & $4 \cdot 7$ & $4 \cdot 9$ & 2. 1 \\
\hline France........... & 1.6 & 0.9 & 2.8 & I. 6 & 2.4 & 0.5 \\
\hline Germany........... & 14.9 & 13.1 & $5 \cdot 0$ & 6.7 & 6.5 & $3 \cdot 5$ \\
\hline Italy........ & 10.7 & II $\cdot 9$ & $5 \cdot 3$ & 10.9 & 12.4 & 10.0 \\
\hline Russia........ & 17.0 & I6.7 & 19.2 & $21 \cdot 3$ & & \\
\hline Spain..... & 6.4 & 9.6 & $5 \cdot 3$ & 10.5 & 11.7 & 11.4 \\
\hline Australia.. & 16.4 & 16.5 & 14.2 & 12. I & I I .3 & 8.2 \\
\hline New Zealand... & I6.0 & $17 \cdot 3$ & 14.2 & I 2.1 & 10.2 & 8.6 \\
\hline Japan......... & $11 \cdot 5$ & I 3.0 & $9 \cdot 9$ & I9.9 ('28) & 14.2 & 13.8 \\
\hline
\end{tabular}

In some respects the comparison of crude birth rates and death rates gives a misleading picture of what is happening $\begin{array}{llllllllllllll}5.3 & 7.2 & 9.6 & 12.8 & 17.1 & 23.2 & 31.4 & 39.8 & 50.1 & 62.9 & 76.0 & 92.0 & 105.7 & 122.8\end{array}$

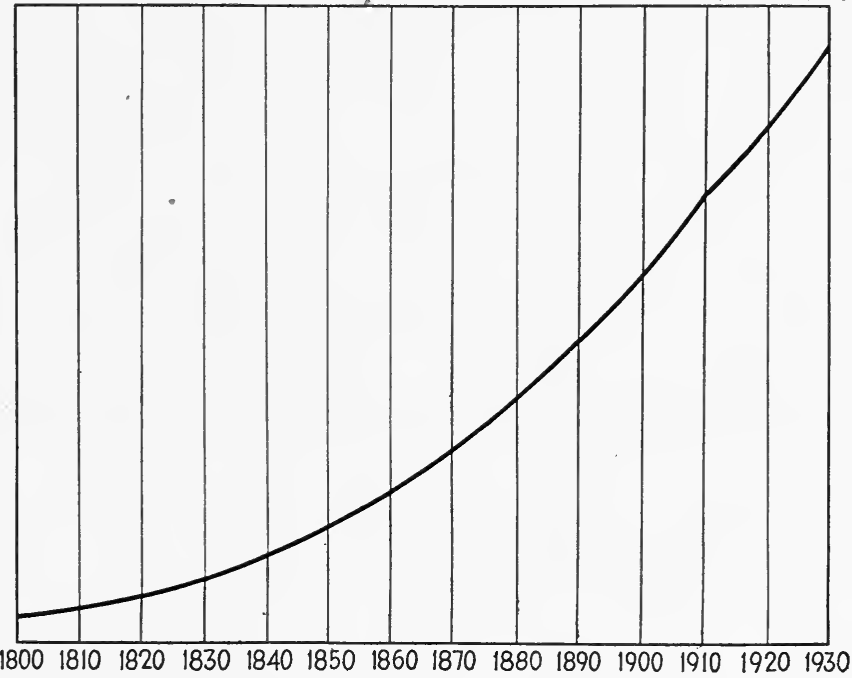

Fic. 75.-Growth of the population of the United States from 1800 to 1930.

to a people. Barring the effects of migration the surplus of births over deaths gives a reliable index of the rate of growth 
at any given time, but it is important to consider that this surplus depends, not merely upon the actual fecundity and health of a people, but also upon its age composition. A country may have more births than deaths and yet not be reproducing with sufficient rapidity to insure the continued maintenance of its stock, even if women should continue to produce as many children and the death rate in each age were to remain unchanged. Let us imagine a population between ages twenty and forty-five in which births were only a little more numerous than deaths. Fifteen years later most of the women would be too old to have many children, and their offspring who had been born in the meantime would not have reached the reproductive age. With the change of age composition there would soon be more deaths than births. Since the actual growth of a population depends so largely on its age grouping it would be of interest to know how rapid its increase would be if the age-specific birth rates and death rates were to remain the same. If the age-specific rates were constant the population would in time outgrow the effect of its changing age distribution and would settle down to a uniform rate of increase which would remain unchanged thereafter. We may therefore speak of the stabilized rate of increase of a people, meaning thereby the rate which would obtain when the influence of its anomalous age composition had been outgrown. The first step in the calculation of such a rate is to ascertain how many daughters would be born to one thousand females. For this we must know how many births occur among mothers in the several age groups. If one thousand mothers produce thirteen hundred daughters, it is not certain that the race will be self-perpetuating, because some of these daughters would die before reaching maturity. If every woman produces a daughter who lives just as long as her mother, the population would neither increase nor decrease 


\section{HUMAN GENETICS AND ITS SOCIAL IMPORT}

in numbers. How many daughters would live long enough to replace their mothers can be ascertained by means of a life table for females. If we know the number of females born to one thousand mothers, we can easily calculate the number of boy babies from the sex ratio at birth (106:100).

The calculation of stabilized rates of increase is of importance because if such a rate is a negative quantity it means that there is inadequate reproduction, even though there may be a considerable surplus of births over deaths. In his volume, The Balance of Births and Deaths, Kuczynski has shown that most of the nations of northern and western Europe are in this situation. While they still have a surplus of births over deaths, were it not for their favorable age composition, they would be actually decreasing in numbers. Even with no further fall in age-specific birth rates or death rates their rates of growth will inevitably diminish. Stabilized rates of increase for several countries are shown in Fig. 76. It is of interest to note that, although in 1930 Germany had a greater rate of net increase than France, her stabilized rate was below that of France on account of her peculiar age composition.

While the decline of the birth rate is going on and for some time afterward the population tends to pile up in the adolescent and middle age groups, and hence it becomes favorable for a high net rate of increase. In France the birth rate has been declining for a much longer period than in Germany and her population has more nearly reached a stabilized condition. As may be seen in Table 26 the net increase of France has varied relatively little for several decades. Northern European countries in which the decline of the birth rate set in during the latter part of the nineteenth century show a much greater decline in their rates of population growth. The inhabitants of these countries increased at a phenomenal rate during the nineteenth 
THE EFFECTS OF POPULATION GROWTH 30I

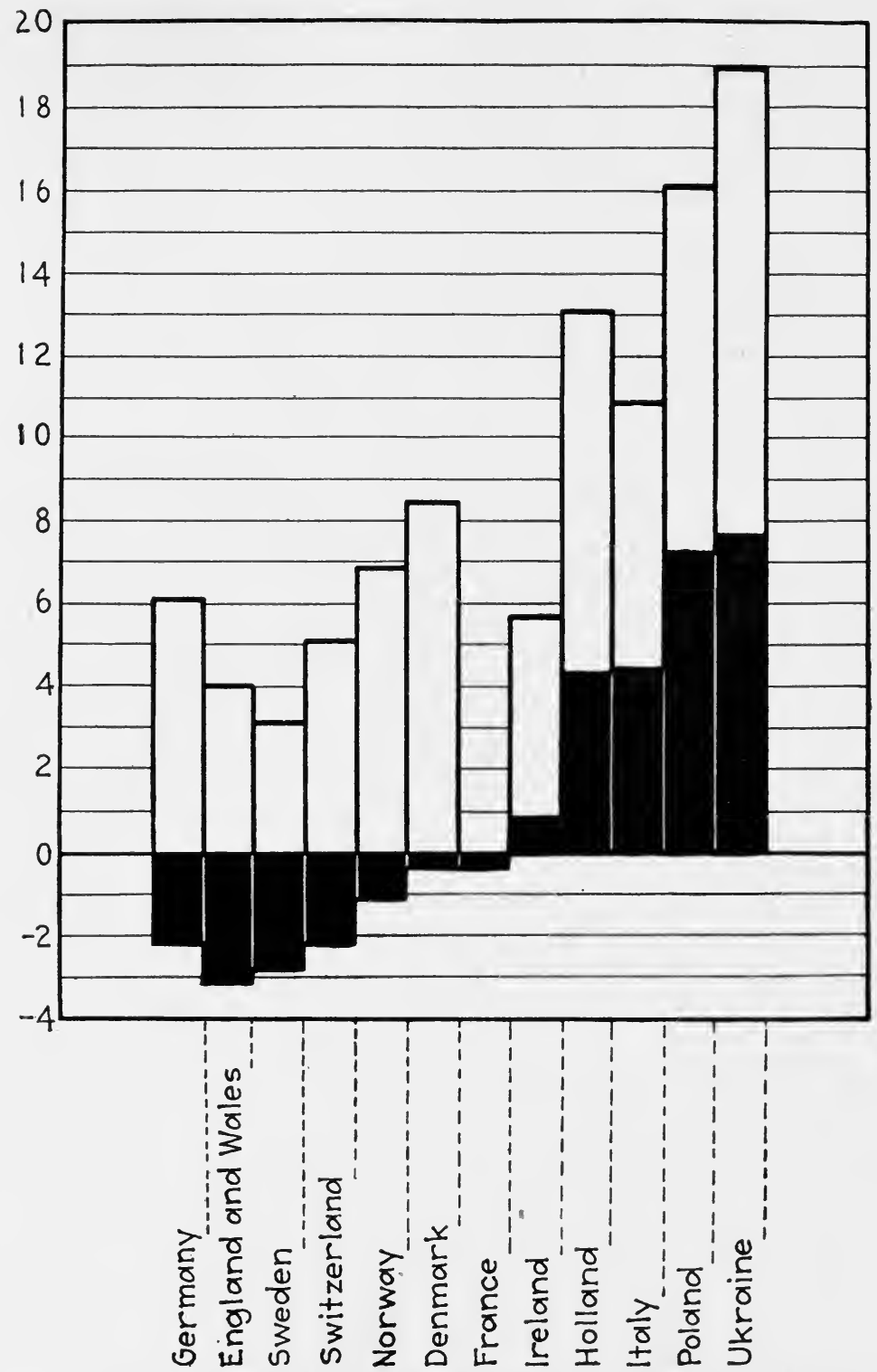

Fic. 76.-Stabilized rates of natural increase (in black) as compared with the excess of births over deaths in several countries of Europe, 1929-1930. (Data from Burgdörfer.) 
century, but they have had their heyday. Only recently have vital statisticians awakened to this fact. They were concerned more over the possibilities of impending overpopulation. It is only since the World War that the stabilized growth of northern and western Europe has passed from a
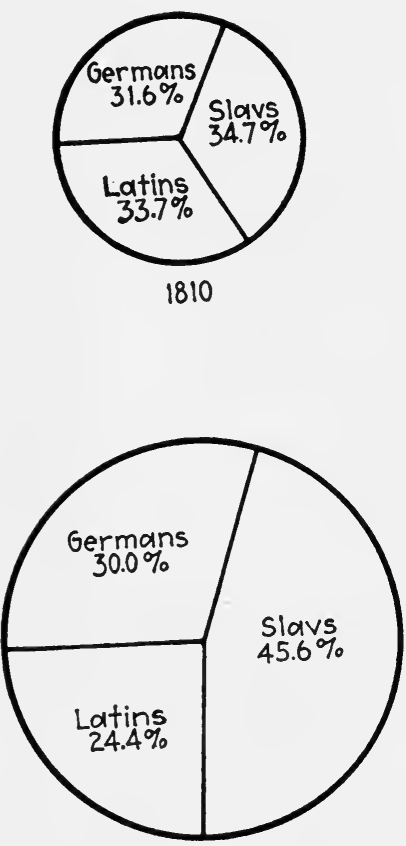

1930
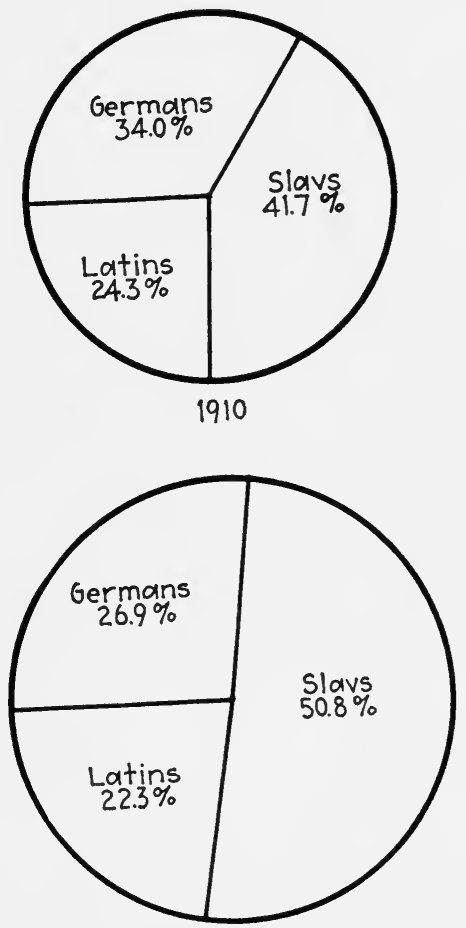

1960

FIG. 77.- Relative increase of Germanic, Latin, and Slavic peoples of Europe, 1910 to 1930 , with the proportions in 1960 if the same relative rates are continued. (Data from Burgdörfer.)

positive to a negative quantity. In part this is due to the loss of men in the war and the disturbances of the sex ratio, but there were other causes of decrease which affected countries not involved in the conflict. One can only speculate as to the trend in the future. Perhaps the birth rate will cease to decline when it has reached a certain stage. One might cite France as a country that has reached a level of 
reproductivity to which other nations are approaching. Population, as Malthus has shown, has a natural tendency to recoup its losses, and, when a decline sets in, the population may automatically respond by a more rapid growth. Many students of population hold that several countries of Europe are overpopulated already and that a little reduction in numbers should be welcomed rather than deplored.

In the United States we have been accustomed to think of ourselves as a young and rapidly growing nation with plenty of room in which to expand and an almost unlimited capacity

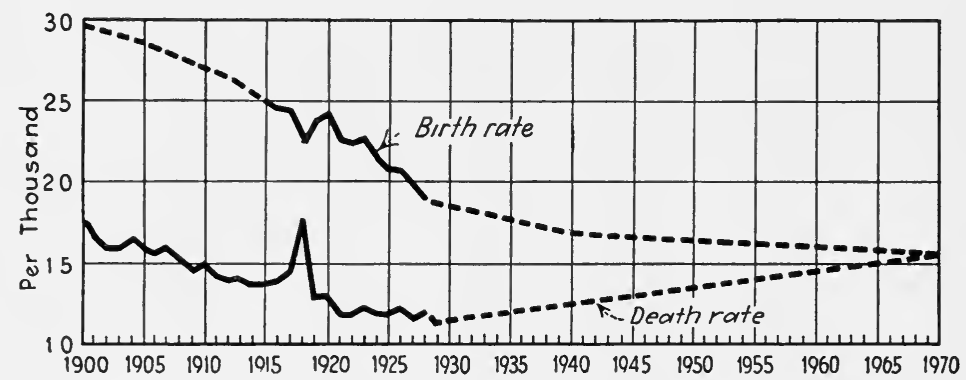

Fig. 78.-Birth rates and death rates in the United States and their probable future trend according to Dublin.

for the production of food. But our country has been rapidly filling up. We are long past the period when free land is to be had for the asking. Most of our tillable land is under cultivation and we are approaching the conditions found in the old world. We have a fairly large but steadily diminishing surplus of births over deaths, but Dublin and Lotka have shown that even in I 920 this was largely due to our favorable age composition. What these authors called the true rate of increase, instead of being I I per thousand as measured by the excess of births over deaths, would be only 5.5 per thousand if we make the proper corrections for age distribution. In 1928 they estimated that the true rate of increase was only I.8. I have endeavored to calculate the 


\section{$30_{4}$ HUMAN GENETICS AND ITS SOCIAL IMPORT}

stabilized rate of increase of the white population of the Birth Registration Area in 1930 and find that it is a little less than 0 , or possibly slightly above if we allow for incomplete registration of births. With the further decline of the birth rate since 1930 our stabilized increase has probably become a minus quantity. In other words, were it not for the

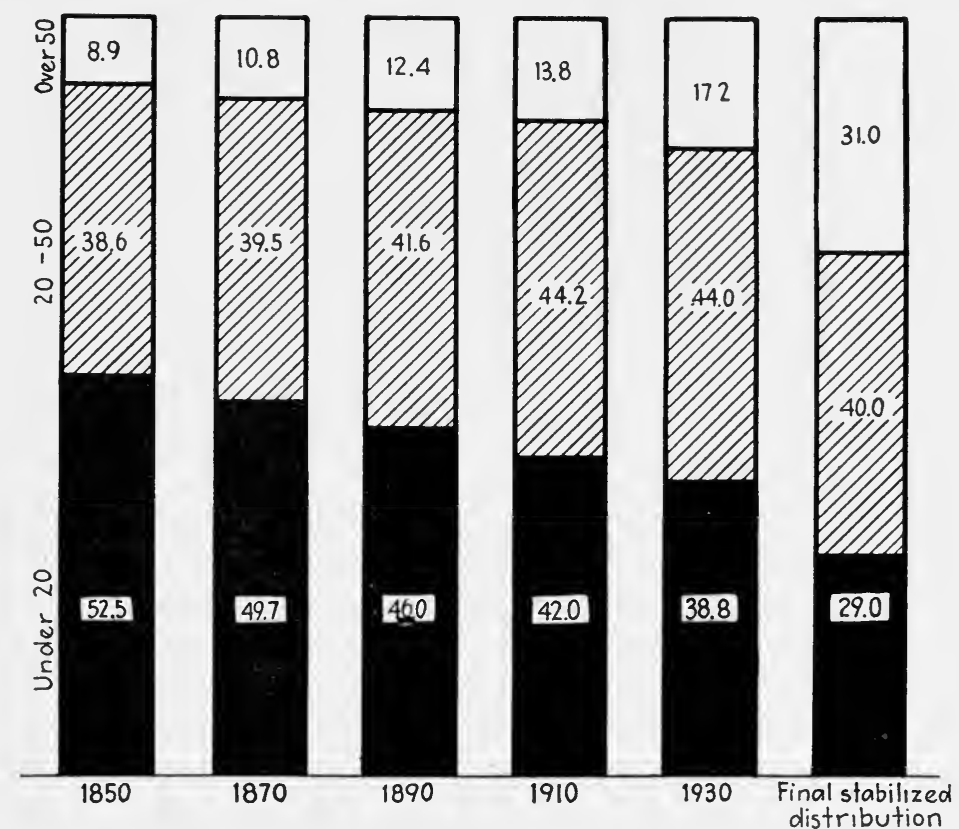

FIG. 79. - The changing age composition of the population of the United States, 1850 to 1930, and the age composition when the population is finally stabilized. The graphs indicate the percentages of the population in the several age groups.

favorable age distribution of our population we would not be increasing in numbers at the present time.

This inadequate reproduction, which has now come to characterize a considerable proportion of the white race, is not so much the product of the unfavorable conditions resulting from overpopulation as it is a consequence of sophistication and the desire not to be encumbered by a numerous progeny. Countries with an inadequate stabilized 
rate of increase are those in which birth control has been extensively practiced for several decades. Some countries which are notorious examples of overpopulation still continue to aggravate the situation by a high net rate of increase. They are under strong pressure to send emigrants to other lands or to acquire territory for their surplus humanity at the expense of their neighbors. Population pressure has long been a strong incentive to migration and to conflict. The white race, before its period of rapid expansion, acquired a large part of this world's territory, a fact which contributed to further its growth. Out of Europe have come millions who have settled most of North America, Australia, and New Zealand, large parts of South America, parts of Africa and Asia, and numerous islands in various parts of the world. The so-called yellow peril has proved to be much less menacing than the white peril has been to the colored denizens of many lands. The enormous expansion of white humanity is one of the great basic events in the recent history of mankind. Not only did the whites desire more land for settlement, but they wanted to control and exploit the wealth of other regions. Highly industrialized countries can support a large population only when they can secure an abundance of raw materials and find markets for their manufactured goods. They naturally desire to obtain the food, coal, iron, oil and other products of foreign lands. They become dependent upon trade for their support, and they naturally endeavor to protect this trade. Where they have the power they are tempted to adopt an imperialistic policy. Nations desire to be strong that they may enjoy a sense of security and not be helpless when their interests are threatened. A nation which is able to grab and exploit new territory may increase its wealth and raise the standard of welfare of its people. It can therefore support a larger population and hence increase its military strength. 
If its population grows unduly the average standard of living goes down, people are tempted to seek their fortunes elsewhere, and the statesmen who control the political destinies of the country look about to secure a larger place in the sun.

Sometimes people suffer grievously from overpopulation without realizing what is the matter with them. The majority of their inhabitants can secure none of the luxuries and few of the necessities of life. They have to work long and hard for a living at the bare subsistence level. They accept their lives of hopeless toil as decreed by fate and go on marrying and begetting children who suffer an appalling mortality in infancy and childhood. Many millions of the inhabitants of this world of ours live under conditions of overcrowding which make life little else than a long struggle against want. If conditions can be remedied by appropriating the lands of their neighbors, the people might easily be persuaded that it would be better for some of them to die gloriously upon the field of battle than to starve in obscurity.

Different rates of population growth have played an important part in shaping the course of human history. They are a chronic source of instability and trouble. The international complications which they occasion have led to numerous wars in the past, and, even with the best intentions on the part of nations to preserve peace, they will continue to present the statesmen of the future with many embarrassing situations. Back of the alleged causes and immediate occasion of the World War were the rivalries for the means of supporting the increasing numbers of the chief participants in the conflict. Unfortunately the same rivalries still persist and are made even worse after the imposition of the terms of peace.

It has become a part of the settled policy of a number of European countries to increase their numbers as a means to 
power. Recent German literature dealing with population problems is filled with lamentations over the low rate of population growth in Germany occasioned by the rapid fall of the birth rate among the masses, and voices eloquent pleas for increasing the population by all possible means. The French, always fearful on account of the dangers arising from their slow rate of natural increase, have vainly attempted to suppress the Neo-Malthusian movement, and to encourage larger families as a means of self-protection. Italy, although poor in natural resources, and having a population that has to be supported on a relatively low standard of living, is endeavoring to increase her population by checking emigration, encouraging births, and securing dominion for expansion in Africa. Militarists the world over are advocates of a high birth rate. When Napoleon was asked, "What woman is worthy of the greatest glory?" he is said to have replied, "She who has produced the largest number of sons for the defense of her country."

The study of population growth not only reveals the real source of many conflicts, but from the humanitarian standpoint it is a subject of the greatest import. The welfare of human beings is closely dependent upon how many of them inhabit a given area. Where people are closely massed together they fall far short of realizing their best capacities for development. Hard toil, ignorance, squalor, vice, and disease are the inevitable consequences of too many people in relation to the means for their support. The life of the masses in such overpopulated countries as China, India, and Japan does not present a pleasing picture. Through industrial development or improved agriculture a country may raise its general standard of living, but if the people should then proceed to breed up to the saturation point, no permanent advantage would result. Should a successful war lead to expansion the benefit might prove to be only 
temporary if it led to no change in the net reproductive rate. There can be no permanent relief to the evils of overpopulation except through the control of population growth.

But countries may suffer from having too few people as well as from having too many. In a sparsely settled region people are advantaged by an increase in numbers. Without a considerable population a society cannot secure the benefits of the division of labor and the exchange of services which afford some of the chief advantages of social life. Up to a certain point labor brings increasing returns as a population grows. The conscious or unconscious recognition of this fact leads countries such as our own was a century ago to make efforts to increase in numbers by inviting immigration. But at some stage the period of increasing returns is followed by a period of diminishing returns in which people have to work longer and harder for what they get. Evidently the sensible thing to do would be to reach a happy medium, or what is called the optimum population. The concept of an optimum population for a given area is somewhat indefinite. Some countries are obviously underpopulated, and others, according to any reasonable standard, grossly overpopulated, but opinions differ as to how the optimum should be defined. A militarist would probably desire a dense population even if it involved a considerable sacrifice of individual welfare. So might an ecclesiastic who considers that the chief function of this world is to supply souls to inhabit the next. Commonly the optimum population is regarded as that which is most conducive to the welfare of the greatest number of persons. Professor Wolfe has defined the optimum as that which gives the greatest per capita income. Professor Fairchild defines the optimum in terms of the standards of living. It has been pointed out that the optimum from an economic 
standpoint, may not necessarily be the same as the optimum from a social or cultural standpoint. It is much more difficult to measure social and cultural values than those dealt with by the economist, but even according to a purely economic criterion it is not a simple matter to ascertain just when the optimum has been reached. Has the economic optimum been passed in the United States? Apparently we are not suffering greatly from overpopulation, and the country could comfortably support many more people with its present resources. W. S. Thompson has shown that in agricultural production we have passed into a period of diminishing returns when farm labor is less rewarding than in former years. Whether the same statement applies to most other branches of industry we do not know, so that our question is one to which economists have not as yet given us a definite answer.

An optimum population varies from time to time with progress in methods of creating and distributing wealth. For an industrial people it is greater than for one engaged in hunting and fishing like the North American Indians. It will also vary according to age distribution and the quality of the individuals. Hence it is difficult to determine very precisely what is the most desirable number of people for a country at any given time. Nevertheless, an approximate solution of the problem is highly important because human welfare is so profoundly influenced by the relation between numbers and means of support.

Populations, as Malthus showed, have an unfortunate tendency to reach their maximum numbers, but human beings have contrived to avoid the drastic checks of Nature to a greater extent than Malthus suspected. The labors of anthropologists and other students of primitive culture have added enormously to the knowledge of the customs of aboriginal peoples that was available in Malthus's day. 


\section{Io HUMAN GENETICS AND ITS SOCIAL IMPORT}

As is shown very convincingly by Carr-Saunders in his excellent volume on The Population Problem the regulation of numbers is widely practiced by the different tribes and peoples of mankind. Infanticide is a common custom among primitive men. It was practiced in ancient Greece and Rome, but it later disappeared almost entirely under the influence of Christianity. Among primitive peoples there is also an extensive practice of abortion. Prevention of conception is less common, but various methods of attaining this end are employed by many tribes. The habit of prolonged lactation in the endeavor to prevent conception during this period is a common custom in primitive society. Besides, many tribes have sexual taboos which prevent the husband from living with his wife for a period following the birth of a child. The number of children a woman may bear is often regulated by tribal custom. If the number is exceeded, the infant must be put to death. Many of the primitive methods of checking natural increase may be crude and brutal, but they are less cruel than those imposed by Nature as a penalty for overmultiplication.

We cannot describe the many ways by which numbers have been regulated by different peoples of the world, and we shall refer to the volume of Carr-Saunders for further details on this subject. The chief point is that mankind quite generally has succeeded in avoiding, in a measure, the more drastic checks to overpopulation. Peoples have by no means always been successful in checking an undue increase in numbers. They have gone at the business in a crude, half-conscious and bungling manner, and have commonly stopped far short of limiting population at the optimum level. But at least they have realized that too many people are undesirable and have acted accordingly.

The modern birth control movement is part and parcel of the effort to prevent the undue increase of numbers 
which has been widely prevalent throughout the races of mankind. It aims to achieve the same end without recourse to infanticide, abortion, restriction of marriage, or sexual taboos. Undoubtedly this movement is chiefly responsible for the reduced rates of population growth in a large part of the white race and to a less extent in some colored peoples. Had it not been for this fact the population of Europe would have been much greater than it is. According to the Neo-Malthusians this would have led to an intolerable degree of overcrowding, reduced the standard of living, and caused a higher death rate and a greater number of wars. The opponents of the Neo-Malthusians might urge that many more people would have enjoyed life, more geniuses would have been born, and the white race would have spread more rapidly and enlarged its domain to the ultimate advantage of humanity in general.

Among the population problems which give the greatest concern to many countries is the ethnic composition of their inhabitants. Most countries have peoples of different stocks in their midst, and the relations of these diverse elements vary all the way from good-natured tolerance to open hostility. The origin of these associations is varied. In many cases it is a result of slavery. The Greeks, Romans, Egyptians, and other peoples of antiquity brought in numerous captives to do the hard work. Later the slaves were liberated and intermarried with and to a large extent outbred their captors, and thus caused marked changes in the ethnic composition of the inhabitants. Slavery is responsible for the presence of Negroes in the United States, South America, the West Indies, and to a less extent in other parts of the globe.

Frequently ethnic diversity is the result of conquest. Few countries have been spared from successive inroads of hostile invaders who have overrun the land and mingled 
with the indigenous population. Witness the population of England with its Britons, Danes, Angles, Saxons, and Normans, or in fact the population of most of the countries of central and southern Europe or of Egypt, Persia, and Mesopotamia, all of which from time to time have been under the yoke of different conquering peoples.

A third factor contributing to the diversified character of populations is immigration. In modern times peaceful penetration has acted more powerfully in this respect than conquest. We owe to it most of the conglomerate nature of our own population which led Theodore Roosevelt to speak of the United States as a polyglot boardinghouse. An extreme illustration of the mixture of diverse racial elements is afforded by Hawaii. The development of the rich resources of Hawaii has led to the influx of Chinese, Japanese, Koreans, Hindus, Negroes, Filipinos, Porto Ricans, Portuguese, and Americans, who together with the native Hawaiians constitute a more diversified and polychrome mass of humanity than is found in any other area of equal size on the earth's surface. The development of cheap and rapid means of transportation has contributed much toward bringing different racial stocks together in the same territory. This cannot fail to have important consequences both biological and social.

The origin of racial diversities within a country often has a great deal to do with the way in which the different elements get along together. Where these elements preserve a strong consciousness of kind and work together in the pursuit of their own interests they are apt to meet with a more or less antagonistic attitude. The Germans are greatly exercised over the presence of the Jews as were the Russians under the Czarist regime. They are also concerned over the presence of the Poles and other Slavic peoples because of 
their portentous fertility. Since the World War and the intensification of the spirit of nationalism which followed it, we have had the spectacle of the Greeks being driven en masse out of Turkey, and of Turks being forcibly expelled from Greece, and more recently of Hungarians being hustled out of Jugoslavia. Even more drastic measures of dealing with unwelcome inhabitants have been practiced by the Turks in Armenia. Nations desire a certain amount of like-mindedness among their inhabitants. Where stocks differ in language, physical appearance, religion, dress, and social customs, antagonisms are apt to be more intense and persistent. Internal discord is a menace to national security in times of conflict and a source of many evils in times of peace. Questions of relative superiority and inferiority of the people living in a common country are apt to be fertile sources of unfriendly relations. In some parts of Europe the Nordics look down upon the Slavs, and in the United States it is common for people of old American lineage to regard themselves as a bit superior to other peoples of the white race and especially to the blacks.

If a nation is composed of very different ethnic elements their relative rates of natural increase become a matter of much concern. This is true whether these elements differ biologically in any significant respect or not. The mode of government, the prevalent religion, and the social traditions - in fact, the whole spirit of a people-may be changed by a process of racial replacement even though the genetic endowments of the stock were practically the same. Good old Puritan Massachusetts is now mainly Roman Catholic in religion as a result of the immigration of people from southern Europe and the emigration of her native American inhabitants to other states. Parallel changes of many other kinds result from the same causes. Hence not only the biological character, but the civilization of a people may be 


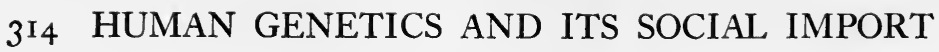

changed through the differential fertility of its component stocks.

The most conspicuous population problem facing the people of the United States is occasioned by the presence of some twelve million Negroes. Beginning with a cargo of slaves imported by a Dutch trader in 1619 our Negro population rapidly increased, partly through the importation of more slaves and partly through its own birth rate. Owing primarily to economic reasons the Negroes became confined mainly to the Southern states, but since their emancipation in 1863 , they have been migrating in ever-increasing numbers into the North and West. During the World War the northward migration became very much greater in volume, and it continued on a large scale during the decade between 1920 and 1930. As a result the "Negro problem" is fast becoming nation wide. The rapid industrial development of the Northern states and the curtailment of the extensive inpouring of cheap labor from Europe conspired to create opportunities for Negro workers who were anxious to improve their economic status. Other influences such as better educational advantages for their children and freedom from certain social restrictions contributed though less strongly to the same end.

Northern Negroes go mainly into the cities where they suffer from a death rate nearly twice as high as that of the native white population. Although there are now more births than deaths among the Negroes of many Northern cities this is demonstrably a consequence of favorable age composition. The stabilized rate of increase is a minus quantity in urban Negroes as it is among urban populations generally. The great breeding ground of our Negro population is the rural south. Relatively few foreign immigrants have gone into the South, especially the rural areas. Had they done so and compelled the migration of Negroes into 
the cities, one of our greatest population problems would be on the way toward solving itself with a considerable degree of rapidity.

The Negro birth rate has always been higher than that of the whites, but it has declined as in the rest of the population and for essentially the same reason. The Negro death rate, however, has been so much higher than that of the whites, that it has more than compensated for the advantage of their higher birth rate. The whites in the United States have increased much more rapidly than the Negroes, but this has been due largely to an extensive influx of fertile immigrants from Europe which for the years immediately preceding the World War often amounted to over a million per annum. With the restriction of European immigration and the fall of the birth rate among the whites the net increase of the two races has come to be more nearly the same.

We cannot calculate the stabilized increase of the Negroes with a high degree of accuracy, but from the available data on births, deaths, and age distribution it is evident that the stabilized rates of the two races are not far apart. A prominent German writer on population problems, Dr. F. Burgdörfer, contends that unless the white population of the United States continues to be recruited by immigrants from abroad, it will gradually be outbred by the Negroes. Prophecies as to population growth, however, are notoriously unsafe, but whatever may be the trend in the future in their struggle for numerical supremacy Negroes and whites in the United States are running nearly a neck and neck race at the present time.

A minor population problem, but one which a few years ago was increasing to an extent that caused a good deal of uneasiness in the Southwestern states, and in California, has been created by immigration from Mexico, as will be 


\section{I6 HUMAN GENETICS AND ITS SOCIAL IMPORT}

further discussed in the chapter on migration. Our foreignborn population of Caucasian extraction forms a considerable proportion of our total inhabitants. Another large proportion consists of the immediate progeny of foreign-born parents. No attempt is made to enumerate those persons whose grandparents or more remote ancestors were born abroad; they are all counted as native-born Americans of native parentage. All European countries have contributed to the making of the American people. The earliest settlers came mainly from northern and western Europe. Later the stream was shifted to southern and eastern Europe, bringing in peoples of different racial extraction, languages, and traditions. A large proportion of our more recent foreignborn population consists of people of relatively low economic and cultural status whatever this may imply, if it implies anything, as to eugenic worth. Obviously, it implies much from the standpoint of the political, economic, and cultural life of the American people. In the course of a few generations immigrant stocks become pretty well Americanized, although the process is delayed by the tendency of alien peoples to segregate in groups where they marry among their own kind and maintain their old traditions, sometimes for generations.

As a rule, the foreign born have a birth rate which exceeds that of the native-born Americans. According to the investigations of the Immigration Commission on the birth rates of native-born and foreign-born inhabitants in sample urban and rural areas, the average number of children per family in I 900 was 4.7 among the foreign born, 2.7 among the native born, and 3.9 in native whites of foreign parentage. In a study of family size among parents sending sons to the University of California I have found that the average number of children was 3.14 in the foreign born, and 2.89 in the native born. Our birth statistics on the average number 
of children ever born to mothers who had given birth to a child during the year uniformly show a higher number for foreign-born mothers. Recently the birth rates of foreign born mothers have fallen off quite rapidly. This is to a large extend due to the fact that, since most foreign-born mothers came here before the restriction of immigration, their fertility is now showing the effects of more advanced age. According to Thompson and Whelpton, the birth rate per thousand women aged fifteen to forty-four in the Birth Registration Area in 1920 was 90 for native-born white women, I36 for foreign-born women, and Ioo for Negro women. In the Birth Registration Area for 1929 the rates for these classes were estimated as 76,92 and 85 respectively. The decline was greatest in the foreign-born women and least in Negro women.

Among the foreign born there are great differences in fertility according to national origin. The Immigration Commission found that the largest number of children per family, namely 4.9, occurred among the French Canadians. Large families are common among the Poles, Southern Europeans, and especially Mexicans, Chinese and Japanese. Among the English, Scotch, Irish, Germans and Swedes births are not so numerous. The birth rates of the native American population of native parentage are especially low in the Northern and Eastern states where they are inadequate for maintenance. In the South and several states of the West, the old American stock is more than perpetuating itself. This stock is largely British and north European in origin, but it seems incapable of maintaining itself when subjected to strong pressure from a foreign immigrant population. What the biological effects of this process of replacement will be depends upon the inherent qualities of the surviving types-a topic which will be touched upon briefly in a later chapter. 


\section{I 8 HUMAN GENETICS AND ITS SOCIAL IMPORT}

\section{Suggested Readings}

Duncan ('29), chaps. I I-I 7. Dublin ('26), chaps. 5-7. East ('23), chaps. 3-6. Knibbs ('28). Kuczynski ('28). Lorimer and Osborn ('34), chap. I. Reuter, ('23), chaps. I-8. Thompson, ('35), chaps. I-8, 24, 25. Thompson and Whelpton ('33), chaps. I, 2, 4, IO, II. Wright ('23).

\section{Questions}

I. What do you consider the most significant principle brought out in Malthus's Essay on Population?

2. Does the means of supporting life increase in an arithmetical ratio as Malthus once stated? If it does not, will it seriously affect the most important features of Malthus's doctrine?

3. If the net rates of increase of two elements of a population originally present in equal numbers were as five to four what would be the numerical proportions of these elements after five generations?

4. What effects did the potato and its diseases have upon the population of Ireland?

5. How did the famine in Ireland affect the population of that country and of the United States?

6. What features of Malthus's doctrine were employed by Darwin in formulating his theory of natural selection?

7. What are the checks to increase among species of animals?

8. In what ways do checks to increase in man resemble those in animals and in what ways do they differ?

9. What nations of the earth are now increasing most rapidly in numbers?

I0. What can you say of the relation between the population of a country and its military strength?

II. What countries do you think are better off because they have a relatively homogeneous population, and what countries suffer from having too great a diversity of ethnic stocks?

12. How will the increasing age of the American people affect school attendance, birth rates, death rates, and general prosperity?

13. If there were fewer people in a country, would there be less unemployment?

14. How does Neo-Malthusianism agree with and how does it differ from the teachings of Malthus? 


\section{CHAPTER XXII}

\section{THE GROWTH OF CITIES AND ITS DEMOGRAPHIC EFFECTS}

$7 \mathrm{HE}$ growth of cities in the nations under Western

1 civilization has involved many changes in the surroundings and customary pursuits of ever-increasing proportions of the population. We live in a machine age which has brought us railroads, steamships, automobiles, telegraphs and telephones, the radio, and countless inventions and conveniences which make life very different from what it was a century ago. The changes that our industrial and technical developments have wrought in everyday life have naturally been greatest in cities, but they have influenced even the remotest and most isolated regions. In recent times the growth of cities has been enormous. A glance at the following table giving the growth of a few of the larger cities of the world shows that the population of most of these cities has more than doubled, and that of others has increased many times over since the middle of the last century.

Table 27.-Population of Selected Large Cities since i 800

\begin{tabular}{|c|c|c|c|c|c|}
\hline & I 800 & 1850 & 1900 & 1920 & 1930 \\
\hline New York. & $79,2 \times 6$ & 696, I 15 & $3,437,202$ & $5,620,048$ & $6,930,446$ \\
\hline London. . & 959,310 & $2,363,34 \mathrm{I}$ & $4,536,267$ & $4,484,523$ & $4,397,003($ I93I)* \\
\hline Paris.... & 547,756 & $1,053,262$ & $2,660,559$ & $2,906,472$ & $2,891,020$ \\
\hline Berlin. . & I 72,846 & 429,217 & $2,712,190$ & $3,804,048$ & $4,242,501$ (I933) \\
\hline Chicago. & $\ldots \ldots$ & 29,963 & $\mathrm{I}, 698,575$ & $2,701,705$ & $3,37^{6}, 43^{8}$ \\
\hline Vienna. & 231,949 & $446,4^{1} 5$ & $\mathrm{I}, 727,073$ & $1,865,780$ & I,868,328 (I925) \\
\hline Philadelphia & $4 I, 220$ & I 21,376 & I, 293,697 & I, 823,779 & $\mathrm{I}, 950,96 \mathrm{I}$ \\
\hline Glasgow... & 77,385 & 344,986 & 761,709 & $\mathrm{I}, \mathrm{O} 34, \mathrm{I} 74$ & I, $, 088,4 I_{7}(1931)$ \\
\hline Moscow... & I 88,654 & 332,878 & $\mathrm{I}, \mathrm{I} 74,673$ & $1,027,336$ & $2,78 \mathrm{I}, 300(\mathrm{I} 93 \mathrm{I})$ \\
\hline Leningrad. & 220,200 & 487,300 & I, 439,6 I 3 & 763,875 & $2,228,300$ (I93I) \\
\hline
\end{tabular}

* The population of "Greater London" in I93I was 8,203,942. This includes the so-called "outer ring." 


\section{HUMAN GENETICS AND ITS SOCIAL IMPORT}

At the beginning of the nineteenth century probably no city contained as many as a million people and probably not more than two, London and Paris, did so in $185^{\circ}$. This enormous growth of urban populations during the last century is obviously a reflection of our rapid industrial development. Formerly agriculture was the principal occupation of the great majority of civilized mankind. Agriculture still leads in Russia, Bulgaria, India, China, and several countries of South America. In I92I the percentage of persons so engaged was $4 \mathrm{I} .5$ in France, 30.5 in Germany, 26.3 in United States, 22.7 in Australia and only 7.5 in England and Wales. The United States has long been a predominantly agricultural country. In 1820 about 72 per cent of occupied persons over ten years of age were engaged in agricultural pursuits, but the percentage has gradually decreased until in 1930 it was only 21.4 per cent.

Until the eighteenth century agriculture had made little improvement since ancient times. Methods of farming were inefficient and wasteful, and little attention was paid to securing profitable varieties of crop plants and farm animals. Many improvements were made in scientific agriculture in the eighteenth century, but they were relatively small as compared with those effected in the century just past. Improved methods of tillage and the development of valuable varieties of grains, vegetables, fruits, and farm animals through selective breeding greatly increased the value of the farm products that could be raised from a given area of land. The invention of agricultural machinery made farm labor much more productive, so that it became possible for a comparatively small number of workers to raise more food than was produced by a much larger number of workers employing the older methods of farming. The result was that relatively fewer people were needed to work on the farms, and hence the surplus rural population migrated 
into the cities to supply the labor required by the rapid growth of industry. Urban migration is a perfectly natural and in fact inevitable consequence of our economic development. City growth and especially the growth of very large cities has been facilitated by the development of rapid transportation made possible through the application of steam and electricity. Steam power has contributed greatly to the development of factories, which for several reasons tend to congregate in the larger centers of population. And in addition to the powerful economic forces leading to

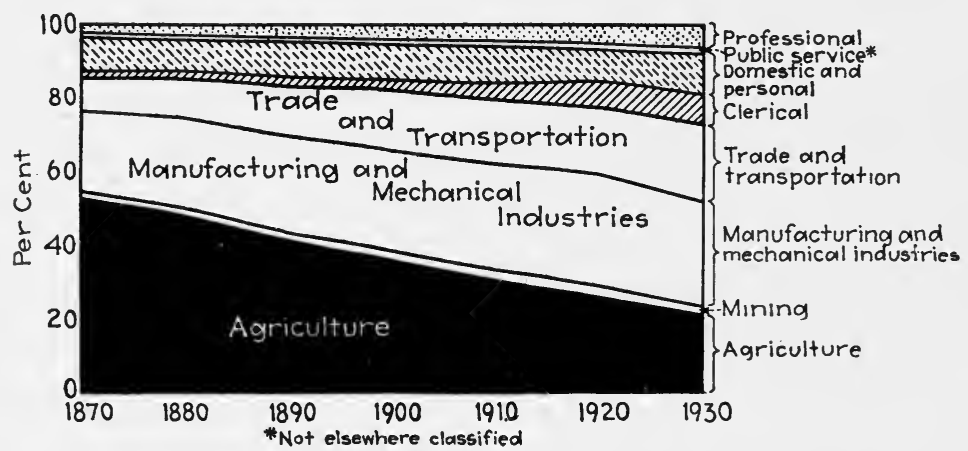

Fic. 80.-The changing proportions of persons over 16 years of age in different occupations in the United States, 1870 to 1930.

urban growth there are other factors such as superior educational advantages, amusements, and the natural gregariousness of the human animal which have conspired to further the same trend.

This unprecedented massing together of human beings in cities cannot fail to have its biological effects. Some of these are fairly easy to ascertain. Others, and among these the more important ones, involve us in the same kind of difficulties that so frequently perplex the student of the social bearings of biology. The more obvious biological influences relate to the age grouping, birth rates, death rates, and ethnic composition of urban inhabitants. We may consider first the matter of age grouping since it is necessary 


\section{HUMAN GENETICS AND ITS SOCIAL IMPORT}

to keep this subject in mind when interpreting the significance of the vital statistics of urban areas. Most cities have relatively smaller proportions of children and old people than are found in the country. The people who migrate to cities are for the most part young adults or persons of middle age who are drawn thither by the prospects of employment. On the other hand, there is a tendency for old people to migrate from the city to the country. As may be seen by the table giving the percentages of the female population of the United States in rural and urban communities, the age distribution of urban females is especially favorable for a high birth rate and a low death rate inasmuch as cities contain relatively more persons in the middle periods of life.

Table 28.-Percentage of the Female Population of the United States, AgEd $0_{4}$ AND 20-44, $1910-1930$

\begin{tabular}{|c|c|c|c|c|c|c|c|c|c|c|}
\hline \multirow{3}{*}{ Ages } & \multirow{2}{*}{\multicolumn{2}{|c|}{ Rural }} & \multicolumn{8}{|c|}{ Cities } \\
\hline & & & \multicolumn{2}{|c|}{$2,500-25, \infty 00$} & \multicolumn{2}{|c|}{$25,000-100,000$} & \multicolumn{2}{|c|}{$100, \infty 00-500, \infty 00$} & \multicolumn{2}{|c|}{ Over $500, \infty 00$} \\
\hline & $0-4$ & $20-44$ & $0-4$ & $20-44$ & $0-4$ & $20-44$ & $0-4$ & $20-44$ & $0-4$ & $20-44$ \\
\hline 1910 & 13.0 & $34 \cdot 7$ & 10.2 & $4^{1} \cdot 6$ & $9 \cdot 7$ & $44 \cdot 6$ & $9 . \mathrm{I}$ & $46 . I$ & 10.3 & $45 \cdot 0$ \\
\hline 1920 & 12.2 & 33.8 & IO. I & $39 \cdot 5$ & $9 \cdot 7$ & 42.9 & $9 . \mathrm{I}$ & $44 \cdot 7$ & 9.8 & $44 \cdot 7$ \\
\hline 1930 & 10.7 & $33 \cdot 3$ & 8.7 & $3^{8} \cdot 9$ & 8.2 & 4 I. 6 & 8.8 & 43.1 & $7 \cdot 7$ & 45.0 \\
\hline
\end{tabular}

Because of the peculiar composition of their inhabitants cities often show a higher crude birth rate than that of the surrounding country. If, however, the birth rate is measured in terms of women of childbearing age (fifteen to forty-five) instead of per thousand inhabitants, the relation is often reversed. In many cases the birth rates of cities are increased by the growing custom of rural women to come to a city hospital to give birth to their children. Where the birth is credited to the city instead of to the residence of the mother, the urban birth rate is raised at the expense of the rural. 
In many cities of the United States, especially in the North and East, the real birth rate tends to be high on account of the large percentage of prolific foreign immigrants. Urban birth rates of foreign-born women have commonly been higher than the rural birth rates of native-born women, and where the population of cities is largely composed of the foreign born the urban birth rate may be higher than the rural. If, however, we compare the birth rates of native-born

Class of

Population

Urban

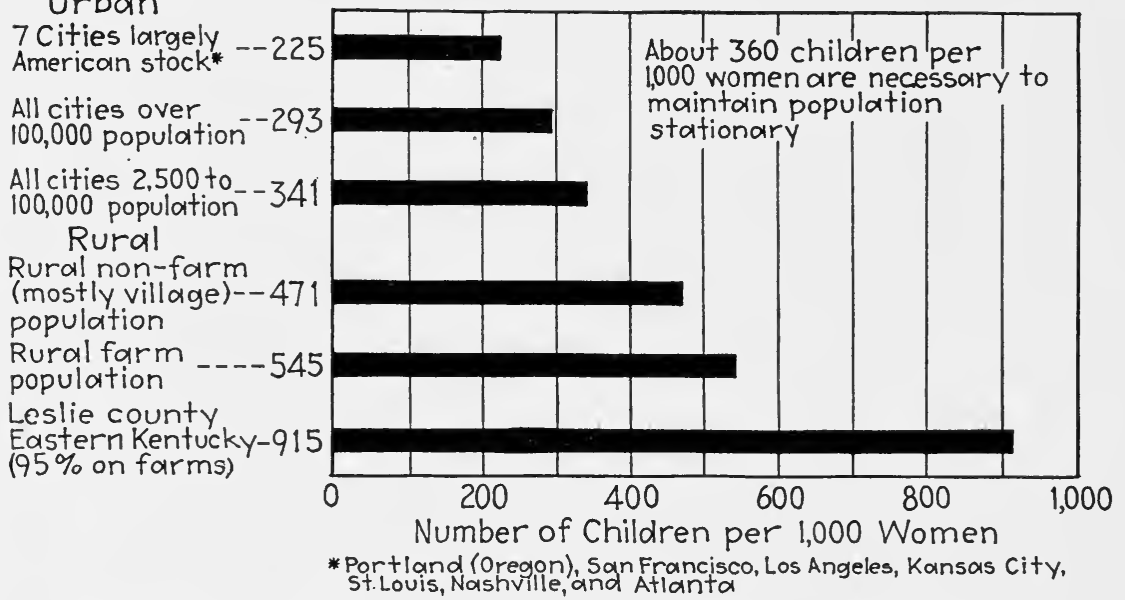

FIG. 81.-Number of children under five years of age per I,000 women, fifteen to forty-four years of age, in the urban and rural areas of the United States in 1930.

and foreign-born women in the same kind of an environment, we find that in both rural and urban areas the foreign-born mothers have a higher fertility.

There can be no question that urban life decreases the fertility of all classes subjected to its influence. Recently the birth rate of foreign-born mothers has rapidly fallen owing to the increasing age of the large numbers who came here before the restriction of immigration.

As is stated in the chapter on the death rate, the mortality of cities is as a rule higher than that of the surrounding 


\section{HUMAN GENETICS AND ITS SOCIAL IMPORT}

country. In many cities there are more deaths than births, and where there are not, the growth of the cities through natural increase is usually due to the favorable age composition of their inhabitants. By and large, cities have been centers of extinction. It is a well-recognized fact that the rural districts supply the real material for population growth. People are produced in the country to be destroyed in the city. Hence were it not for the continual migration from the country to the city many urban populations would soon decrease in numbers. According to Whelpton, the "true," or stabilized rate of natural increase in nine of the Table 29.-Children 0-4 Per Thousand White Women, 20-44, in the United States IN I920

\begin{tabular}{l|c|c|c|c|c}
\hline & \multirow{2}{*}{ Rural } & \multicolumn{4}{|c}{ Cities } \\
\cline { 3 - 6 } & & $2,500-10,000$ & $10,000-25,000$ & $25,000-100,000$ & Over 100,000 \\
\hline $\begin{array}{l}\text { Native born. } \\
\text { Foreign born. }\end{array}$ & $\begin{array}{l}720 \\
998\end{array}$ & $\begin{array}{l}477 \\
873\end{array}$ & $\begin{array}{l}434 \\
861\end{array}$ & $\begin{array}{l}390 \\
766\end{array}$ & $\begin{array}{l}341 \\
679\end{array}$ \\
\hline
\end{tabular}

largest cities of the United States in 1928 was a negative quantity ( $-5 . \mathrm{I}$ per thousand), and, according to Lorimer and Osborn, "Effective fertility in $19^{2} 5^{-29}$ in the urban areas appears as only 5 per cent below replacement quotas in Maine; in other states it runs: Vermont, I I per cent below; Massachusetts, I 8 per cent below; New York, 27 per cent below; Indiana, 7 per cent below and Illinois 23 per cent below."

The stabilized-rate of natural increase is likewise inadequate in the larger cities of Europe. This is especially true of Paris, London, Vienna, and Berlin. According to Burgdörfer, Berlin would require a birth rate of 17.4 for maintenance, but in 1927 the birth rate had fallen to the remarkably low level of 9.9 , a rate which would be still further reduced to 7.6 in a stationary population. In the same year the birth 
rates were I I.I in Dresden, I I.6 in Frankfurt, I 2.0 in Munich, I2.0 in Stuttgart, I2.2 in Hamburg-all far below the maintenance level. Hansen has remarked that if the rural population of Germany consisted of Negroes, while the cities were inhabited by the whites, it would not be long before the entire population would be black. In the long run the character of the population of a country is determined by the kind of people who inhabit the land.

We may regard cities, then, as constituting a continual drain upon the population of a country. As they increase in size the drain becomes ever greater in magnitude. The growth of cities has become an important factor in the decreasing rate of population growth in the more highly civilized peoples of the earth. It would be very desirable, therefore, to know how the genetic qualities of migrants to the cities compare with those of the people who remain in the country. Galton has expressed the view that the more intelligent and enterprising individuals of the rural areas tend to seek their fortunes in the cities and thus lead to the genetic impoverishment of the rural population. For several reasons this seems to be a fair conclusion, but it is difficult to secure the data required to subject it to a critical test. In some regions at least, the cities may attract the vagabonds and ne'er-do-wells to whom work on the farm makes little appeal. In the United States and most other countries no official records are kept of the comings and goings of people; hence we must rely upon special studies of individual areas for whatever evidence may be obtained upon this problem. Unfortunately the evidence is not so plentiful or so convincing as could be desired. We can mention only a few of the studies which have been made. There is little doubt that in isolated regions in New England and the Middle Atlantic States which have become reduced in population on account of poor soil, communities have 


\section{HUMAN GENETICS AND ITS SOCIAL IMPORT}

suffered from the loss of their more intelligent members. In some of these the percentage of mental deficiency is high. The Hill Folk studied by Danielson and Davenport, and later by Miller, affords an illustration of a community characterized by poverty, mental deficiency, physical defect, and unfortunately also by high fertility for several generations. There are isolated communities of "poor whites" in relatively barren regions of the South where they have lived and inbred for many years. In Colvin Hollow, Virginia, studied by Sherman and Henry, we have an area of declining economic productiveness associated with low mental status among the inhabitants. When the region commenced to deteriorate "the more energetic began to desert the log huts and migrate to other parts of the state. A few were able to establish themselves and quickly forgot their mountain associations. Many failed and returned."

The studies of Gee and Corson on Rural Depopulation in Certain Tidewater and Piedmont Areas of Virginia showed that there was a distinct tendency for the more educated persons to migrate away from the region. Of the 1,636 whites studied, 40 per cent of those who had attended high school, 46 per cent of those reaching the seventh grade, and 55 per cent of those who had gone to college or special training schools had migrated, as compared with 35 per cent who had reached the fourth grade or less, and 34 per cent of those who got no further than the fifth or sixth grade. The same relation between education and migration is shown also by the I,253 Negroes included in the investigation. Of course, failure to obtain a certain grade in school may be due entirely to circumstances having no relation to innate ability, but several studies have shown that those who drop out of school in the lower grades are frequently characterized by a low average I.Q., and it is reasonable to 
infer that a part of this failure is the result of a real inherent deficiency in intelligence.

Several studies on the relative I.Q.'s of urban and rural children show that the latter as a rule make the lower scores. Jones, Conrad, and Blanchard in a study of rural and urban children in northern New England conclude that while "a rural child moving to the city would increase his intelligence-test scores merely as a result of changed environment . . . it is unsafe, however, to infer that the average retardation of rural children is chiefly due to environmental factors." The authors conclude that "the most likely estimate" is that about half the average difference between the scores of urban and rural children "is attributable to factors other than those derived from the social and educational environment."

How far the average differences in the I.Q.'s of city and country children are due to the superior educational advantages of city schools is not easy to determine. In attempting to estimate the character of urban migration, I am inclined to place more emphasis upon the fact that individuals graduating from high schools and colleges have a marked tendency to migrate from the country into cities where they find careers open to their talents. The schools are selective agents in which persons with low native ability are weeded out at various stages of their progress, and those who survive to pass through the upper grades doubtless have a somewhat higher measure of native ability than the general school population. Sorokin and Zimmerman have concluded that there is a "net law of rural-urban social selection" according to which "the cities attract the extreme while the farms attract and hold the mean strata in society." There is evidence, however, that the nature of selective ruralurban migration is subject to much variation in different regions, and at present we do not have a sufficient number 


\section{HUMAN GENETICS AND ITS SOCIAL IMPORT}

of critical studies to enable us to tell what the general influence of urban selection is upon the population of any country. If, on the whole, it exercises a dysgenic effect, as seems not unlikely, it is highly important that we obtain more detailed knowledge of its workings. Should the discouragements which those engaged in agricultural pursuits have had to face in recent years lead the more intelligent to abandon farm life to be replaced by people of lower standards of living and a lower grade of intelligence, the native quality of the population will surely decline. Since those who till the soil are the chief source of population growth, it should be the policy of every nation to see that its rural inhabitants consist of physically and mentally sound and healthy stock.

\section{Suggested Readings}

Lorimer and Osborn ('34), chaps. 2, 4, pp. 59-66, 84-96. Thompson ('35), chaps: 17-20. Sorokin and Zimmerman ('29). Gee ('31 and '33).

\section{Questions}

I. What proportion of the population is foreign born and what proportion consists of native Americans of native parentage in the following cities: New York, Boston, Chicago, Philadelphia, Atlanta, New Orleans?

2. Are the populations of cities more or less homogeneous than those of the surrounding rural areas?

3. Compare the age composition of the following cities with that of the states in which each is situated: New York, Chicago, Detroit, Pittsburgh.

4. Look up the sex composition of a half dozen large cities. What do you infer as to the cityward migration of the two sexes?

5. How is urban selection likely to affect the future of the Jewish people?

6. Why are people in cities less susceptible to certain contagious diseases than the inhabitants of the country?

7. How do city and country compare as to the physical development of their children?

8. From the standpoint of maintaining health what are the advantages and the disadvantages of large cities as compared with rural areas? 
9. In what ways do the industries of a city determine the selective character of urban migration?

I0. How is urban life in the United States tending to influence the relative rates of increase of native-born and foreign-born stocks?

II. Why do chambers of commerce in cities endeavor to promote increase in numbers? Is such increase a benefit to all classes of city dwellers? Are there any classes who are disadvantaged as a result of city growth?

I2. On the whole, do you think that cities attract the most intelligent or the least intelligent of the rural population?

13. What do you think will be the eugenic effect of the present depression in agricultural industry?

14. Can you think of any feasible methods by which the state could encourage people of superior natural endowments to live in the country? 


\section{CHAPTER XXIII}

\section{THE BIOLOGICAL EFFECTS OF MIGRATION}

T THE continuous replacement of one stock by another 1 which has gone on during the evolution of the human species, the migration of peoples has played a very important role. The invasion of other territories may or may not involve war, but it is always productive of a certain amount of racial change. Formerly peoples often migrated en masse. In the raids of the Huns, Tartars, Goths and Vandals, and the exodus of the Children of Israel from Egypt, the men, women, and children with whatever chattels they possessed sallied forth to seek their fortunes in new territories. The causes of these migratory movements are varied. Overpopulation, the pressure of hostile invaders, the failure of the food supply as a result of drought or other causes, and the allurements of the wealth and fertile soil enjoyed by other peoples, are among the more common incentives which have led people to try to possess themselves of other lands. As civilization has advanced, these mass movements of whole peoples have given way to the migrations of individuals or small groups which for the most part involve no breach of peaceful relations.

Peoples sometimes leave their home land on account of political oppression or the desire to secure freedom of religious worship, but the numbers influenced by such considerations are relatively small; the prevailing motives are and have always been economic. Countries which are overpopulated often send forth many emigrants, and sparsely settled countries which offer attractive economic 
opportunities tend to receive many immigrants. The movements of peoples, like the movements of currents of air, are from areas of high pressure to areas of low pressure.

Migrations have been greatly facilitated by the development of cheap and rapid means of transportation, as is well illustrated by immigration into the United States. Agents of steamship companies have permeated Europe to secure possible passengers, and have set forth the great advantages awaiting the worker in this land of plenty. Crossing the ocean is a very different matter now from what it was two centuries ago. Passage by steerage is cheap and lasts but a few days. Many thousands come to the United States when work is plentiful and return to their native country when it is scarce. This is especially true of the immigrants from southern Europe, and to a certain extent of immigrants from Mexico.

Of all countries of the world the United States has received the largest number of immigrants from other continents. Between I820 when the number of incoming aliens was first recorded and I929, it is estimated that about $38,000,000$ entered the United States, the majority of whom, about $30,000,000$, have remained permanently. In addition to the immigrants entering the United States others have gone into Canada, Mexico, Central and South America, so that the total migrating to countries of the Western Hemisphere since 1800 is considered to be about $57,000,000$, most of whom came from Europe. Besides, Europe has sent numerous migrants to Australia, New Zealand, Africa, and many other parts of the world. The total exodus from Europe during the nineteenth century has been estimated at approximately 60,000,000. And this during a period when the population of Europe was undergoing an unprecedentedly rapid growth. The greater part of this vast movement of peoples was an orderly and peaceful migration into countries 


\section{HUMAN GENETICS AND ITS SOCIAL IMPORT}

in which the newcomers were welcomed or at least not actively opposed. For the most part also it sprang from the desire of workers to better their living conditions by settling in comparatively new countries with many undeveloped natural resources.

During the nineteenth century countries of other continents have sent forth emigrants, but the numbers do not compare with those coming from Europe. One of the countries most prolific in emigrants is China. Frequently one to two million Chinese have migrated into Manchuria in a single year, although to a certain extent this may be regarded as an internal migration. But Chinese are found quite plentifully in the Philippines, East Indies, Formosa, Siam, Hawaii, parts of South America, and the United States. Japan which, like China, is greatly overpopulated has sent forth emigrants into Korea, Manchuria, the Philippines, Hawaii, and also into the United States until the number was gradually reduced following the so-called gentlemen's agreement. From India have come immigrants who have gone to South Africa, Malaya, and many islands of the Pacific, but the number, two to three millions, is insignificant as compared with the millions living in the home country.

Although Asia has several times the population of Europe and a vast proportion living under conditions of overcrowding which create a powerful incentive to send her people in to other lands, relatively few Asiatics have migrated beyond her borders. Europe has been in a very different situation. Owing to her highly developed civilization, the better organization and equipment of her fighting forces, and especially her naval power, which gave her undisputed control of the sea, Europe was able to seize upon large areas of the earth to colonize and exploit. The most successful nation in the acquisition and peopling of new territories was Great Britain, whose dominions almost encircle the 
globe. A leading role was once played by Spain, but she has now lost most of her foreign possessions. To a somewhat less degree extra territories have been acquired by the French, Portuguese, Dutch, Germans, Danes, and Belgians. The Europeans have grabbed while the grabbing was good. The result has been that Asiatics are kept out of extensive regions which they might have occupied. They look with envious eyes upon thinly populated Australia and New Zealand whose inhabitants view with apprehension the increase of the teeming millions of their Asiatic neighbors, and take all possible precautions to keep their population white. Asiatics are accordingly excluded from Australia and New Zealand in the fear that, once the gates are opened, the population would eventually be overrun by incomers with low standards of living who would gradually supplant the whites in the struggle for existence. Asiatics likewise find themselves debarred from the United States and Canada, the two outlets most favorable for their expansion. South America as yet offers few attractions, and her countries would probably restrict Asiatic immigration if it threatened to increase unduly in volume. Africa, which is almost completely under European control, offers limited opportunities for immigration from Asia. Many Hindus have migrated into the parts of Africa controlled by the British, but they are now excluded from South Africa, and their entrance into Kenya is discouraged; thus the volume of immigration from India has become reduced. Hindus are not particularly desired in most other British possessions; hence they find relatively few places open to them.

As countries increase in population they show a tendency to restrict immigration. When the United States was a young and sparsely settled country it welcomed the foreigner with open arms. There were vast stretches of fertile land 


\section{HUMAN GENETICS AND ITS SOCIAL IMPORT}

to be tilled, forests to be cut, and mineral wealth to be dug out of the ground. We needed people to develop the sources of wealth which abounded on every hand. We aspired to become a populous, rich, and powerful nation. We were persuaded, rightly or wrongly, that we enjoyed a greater degree of political and religious liberty than the people living in the monarchies of the old world, and we were glad to offer them a haven of refuge from their oppressors. So long as we were convinced that the immigrant was a valuable economic asset we easily persuaded ourselves that it was a very noble and gracious act to encourage his coming. Hence we opened our doors freely to all comers. The famine in Ireland during the forties sent great numbers of Irish to this country, and there was soon a considerable immigration from Germany, the Scandinavian countries, as well as Great Britain. The great mass of European immigration in the first three-fourths of the nineteenth century as well as in the preceding century was of northern European origin and therefore made no marked change in the ethnic composition of the American people. There was much intermarriage between the incomers of different nationalities and between these and the native-born Americans. The immigrant stocks proved to be thoroughly assimilable in every way.

In the latter part of the nineteenth century and during the earlier years of the twentieth the shift of the tide of immigration from northern to southern and eastern Europe brought in numerous Italians, Poles, Austrians, Greeks, Hungarians, Russians, and Bulgarians together with a very much reduced influx of the older immigrant stocks. Unlike the peoples from northern and western Europe the majority of the newer immigrants lodged in cities, many of them never budging after their arrival at New York, Philadelphia, or other ports of entry. The more recent immigrants were 
mostly unskilled laborers who went into the mines and factories or engaged in menial employments in the cities. They afforded a plentiful supply of cheap labor, and their coming was encouraged by employers, who wanted workmen of low standards of living, the kind that would be satisfied with very moderate wages. As compared with the older immigration the southern European stocks were characterized by a high degree of illiteracy. From 1899 to 1909,

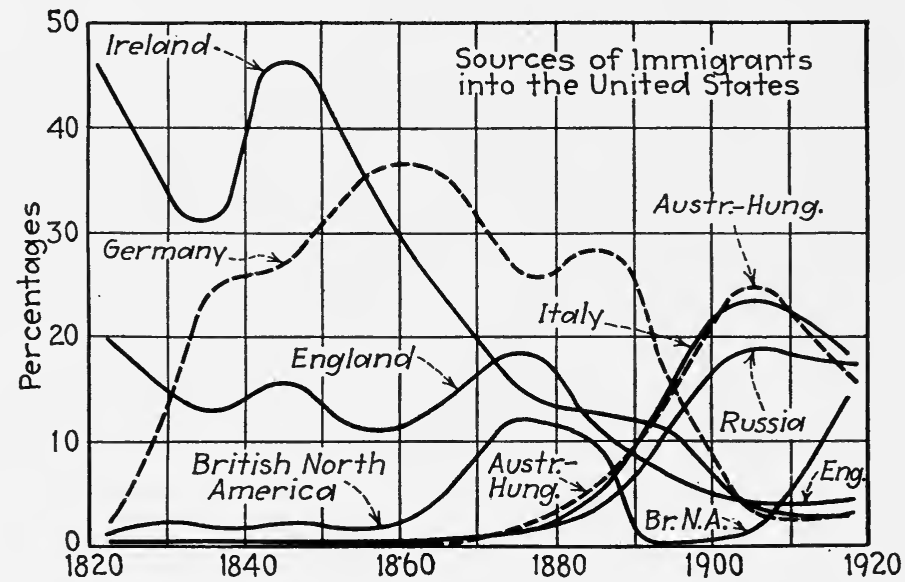

FIG. 82.-Proportions of immigrants entering the United States from 1820 to 1920 . The vertical scale indicates the per cent that each nationality constitutes of the total immigrants. (After Harper. Courtesy of Eugenics.)

54.2 per cent of the immigrants from southern Italy were unable to read or write. The percentage of illiteracy in the newer immigration during this decade was 35.8 as compared with 2.7 in the older immigrant stocks. On the whole, the newer immigrants, although they included many people who formed valuable additions to our population, proved to be rather more difficult to assimilate than the old. During and after the World War many efforts were devoted to the Americanization of these foreign elements in the hope of converting them more rapidly into cooperative American citizens. The tremendous volume of immigration imme- 


\section{HUMAN GENETICS AND ITS SOCIAL IMPORT}

diately preceding the war rendered these efforts difficult of accomplishment, and it came to be perceived that the presence of so many illiterate aliens, whatever may have been their native qualities, created many social, educational, and other problems. The result was that many were convinced that a very large number of such aliens was not an unmixed blessing. American laborers felt the competition of the cheap labor from southern Europe, and they exerted their influence in favor of the restriction of immigration, but their political efforts were opposed by organized groups of employers in the coal and iron industries, meat packing plants, railroads and steamship companies, and other powerful organizations which spent millions in the endeavor to sway public opinion in favor of the unrestricted immigration of aliens.

The volume of immigration was much reduced during the World War, but for a few years after the Armistice was signed it increased rapidly, reaching 802,000 in I92I, and threatening to assume embarrassing proportions if suffered to continue without restriction. Accordingly, Congress was led to pass a law in I92I limiting the number of aliens admitted to 3 per cent of the number in the several nationalities in the United States in I9IO. In 1924 the number was reduced to 164,667 and in 1929 it was further reduced to I53,7I4. During the financial depression there has been an unusual return movement, so that the departures have exceeded the number entering the country.

The reduction of immigration from northern and western Europe was doubtless due in part to the decline of the birth rate in these regions. Another factor was the industrial development of these countries, which created more favorable conditions of life, so that workers would not greatly improve their lot by coming to the United States. Also the supply of free land that formerly attracted so many northern 
Europeans was exhausted and the immigrants would have to become laborers on farms or in mines or compete with southern Europeans in the cities. Among the southern and eastern Europeans there were fewer employments to absorb the growing population. The birth rate continued to be high and there were greater temptations to seek better opportunities in other parts of the world.

The establishment of the quota system limiting the proportion of immigrants who could enter from any one country led to a great reduction of immigration from southern Europe. The aim of the assignment of quotas was to preserve approximately the existing ethnic composition of the American people. This was accomplished in accordance with a general rule without making invidious discriminations against any European nation. The quota system was not applied to nations in the Western Hemisphere, and immigrants from Canada, Mexico, the West Indies, and Central and South America could enter in any desired numbers, subject only to the literacy test and a small fee required of all incoming aliens. The reduced numbers of laborers coming from southern Europe had the effect of stimulating immigration from the countries of the Western Hemisphere, and especially from Mexico. The Mexican invasion threatened to give rise to race problems second only to those created by the presence of the Negro. The great majority of Mexican immigrants consisted of peon laborers mostly of Indian blood. Owing to the demand for cheap labor in the large farms, mines, and various industries in the Southwest, Mexicans poured over the border in ever-increasing numbers. Up to I 900 Mexican immigration had been almost negligible in volume, seldom exceeding five hundred per annum. In 1908 the number rather suddenly increased from $9^{1} 5$ to 5,682 . By 1924 the number reached its maximum, namely 87,648 , and in 1925,1926 , and 1927 the numbers were 
$32,378,42,638$, and 66,766 respectively. In large areas of the Southwest Mexicans performed most of the unskilled labor. The pick and shovel men on the Southern Pacific and Santa $\mathrm{Fe}$ Railroads were said to consist exclusively of Mexicans. Being used to low standards of living the Mexican laborers were content with low wages and poor living quarters, which white laborers would not accept. They proved to be tractable and fairly industrious, and on the whole more profitable to employ than the casual white workers who could be obtained for the wages offered. The result was that they drove white labor out of one industry after another in the Southwest. As their numbers increased, Mexican laborers pushed farther to the North and East, and Mexican quarters grew up in Omaha, St. Louis, Chicago, Pittsburgh, and other large cities. In parts of the South, Mexicans replaced the Negroes in several employments and proved to be one of the causes of their migration into the Northern states.

Many of the employments of Mexicans are seasonal, and they trek with their families from one place to another as opportunities for employment determine. Frequently they are unemployed and become a burden upon charity. Although they possess many attractive qualities, it cannot be said that they contribute much to the cultural life of the communities they enter. Politically they are largely under the sway of bosses who determine how they shall vote. Their record for crime is distinctly bad, and in many communities they create embarrassing social and educational problems. Their birth rate is very high and in many localities the majority of the children in the schools are Mexicans. The studies which have been made on the intelligence of Mexican children indicate that their average I.Q. is low, but to what extent this is due to the disadvantages of their upbringing instead of their heredity is not determined. At least the Mexican peon has shown little evidence of intellectual 
superiority. But granting that his defects are cultural and not genetic it remains true that the average Mexican is singularly slow of assimilation. Even today there are numerous Mexicans in New Mexico, descended from the population of the territory when it was taken over by the United States, who are unable to speak the English language; and even in the state legislature the proceedings have to be published in both English and Spanish for the benefit of constituents who are unable to read the English language.

The race mixture which occurs between Mexican peons and whites is largely on the basis of illegitimate unions between white males and Mexican females. There is a good deal of miscegenation between Mexicans and Negroes and between Mexicans and American Indians. Some Mexicans are of pure Spanish extraction, but these constitute a small minority. More have some admixture of Spanish or other white blood, but the rank and file of Mexican laborers are almost pure Indians.

Many people in the regions in which Mexicans have come to be numerous made efforts to secure the curtailment of Mexican immigration. A bill, the Box Bill, was introduced into Congress with the aim of putting Mexico on the same quota that applies to the nations of Europe. In the discussions of this bill before both the Senate and the House Committees on Immigration the large financial interests who profited by cheap Mexican labor were represented by numerous and able lobbyists who contended that many industries were dependent upon the Mexicans, that white labor could not be secured for the work that had to be done, that the Americans who were forced out of their jobs went into better paid positions, that most Mexicans were only temporarily employed and subsequently returned to their native country, and that the Mexican immigrants were on the whole peaceable citizens and good neighbors who would 
be a valuable acquisition to the country. The chief argument was that the Mexicans were a valuable economic asset and that their exclusion would have a disastrous effect upon many industries.

On the other hand, those opposed to Mexican immigration contended that the Mexicans were the reverse of a financial asset to the working classes and the renters of land inasmuch as they were driven out by Mexican competitors. Mr. McKemy, Commissioner of Labor in Texas, stated that "Mexican labor has supplanted native American labor to such an extent that today fully 75 per cent of the common or unskilled labor in my State is performed by Mexicans. This deplorable situation can be traceable to only one cause and that is the inability of the American to compete with the Mexican in living standards." American renters and small farmers were almost completely eliminated from certain sections of the Southwest by Mexican competition, and the schools were largely filled by Mexican children.

The contention that Mexicans return to their native country proved to be a specious argument. It is true that there is much migration back and forth across the border. But the very rapid increase of the Mexican population in the Southwest, the large numbers of Mexican children in the public schools, and the portentously high birth rate of Mexican families showed only too clearly that we were acquiring a new race problem of no small proportions. Owing to the difficulty of adequately guarding the long border between the two countries, numerous Mexicans have entered illegally. Those who were unable to pass the simple literacy test imposed or those who wished to avoid the payment of the visa fee and head $\operatorname{tax}(\$ 24)$ were often led to adopt the simple expedient of paddling across the Rio Grande or crossing through the brush into New Mexico, Arizona, or California. The Commissioner of Immigration estimated that 
those entering illegally were as many as those coming through the regular channels. The census estimate of the number of Mexicans who were born in Mexico and were living in the United States in I9IO was 221,915; in I 920 it was 486,4 I8; thus showing a gain of $264,5 \circ 3$. The excess of admissions over departures of Mexicans between 1910 and 1920, according to the reports of the Commissioner General of Immigration, was 163,105 . Evidently more than 100,000 must have entered without giving an account of themselves. By $193^{\circ}$ the foreign-born Mexicans amounted to 616,988 and the total Mexican population was $1,422,533$. Since the depression large numbers of Mexicans finding no employment have returned to their native country. In fact, since 1930 more have departed than have come in. But whenever a demand for labor arrives Mexican immigration will again assume large proportions unless steps are taken to keep it within bounds.

I have dwelt upon Mexican immigration because it presents' a typical illustration of the working of a process that has repeated itself many times in the course of history. People of inferior culture and low standards of living are induced to enter a country because their labor is cheap and they contribute to the prosperity of the employing classes. The wealth of a country is increased by. their coming. The classes who are interested in cheap labor are politically powerful, and governments, which are usually concerned only with the more obvious economic bearings of the questions with which they deal, commonly disregard everything else. The effect of such immigration upon the welfare of the laboring classes of a country is as a rule given scant consideration unless these classes have sufficient political power to make their influence felt. Even the Immigration Commission, which was appointed to make a study of all aspects of immigration into the United States and which reported its findings in twenty-eight substantial volumes, 


\section{HUMAN GENETICS AND ITS SOCIAL IMPORT}

expressed the view that immigration is a purely economic problem. Until recently it has been treated as such in practically all the legislation which has been enacted on the subject. The only discriminations made on the basis of quality were to forbid the entrance of the insane, epileptics, imbeciles, criminals, prostitutes, anarchists, or people liable to become a public charge. But up to a few years ago our administration of these regulations was almost unbelievably lax. These measures, so far as they went, doubtless had a eugenic effect. They have gradually become wider in scope and more effectively enforced. Aside from the exclusion of Asiatics the quota regulations represent the first thoroughgoing effort to regulate immigration on the basis of national origin. Incidentally this meant to a certain degree the control of immigration on the basis of racial stock, and whether or not it is eugenic or dysgenic in its influence depends upon the innate characteristics of the various peoples who enter the country.

Properly to evaluate the genetic characteristics of our incoming aliens amid their differences in language, traditions, degrees of education, and the effects of varied environmental influences is no easy task. The first extensive collection of data bearing on this problem was supplied by the Army Mental Tests during the World War. Mental tests were administered to over a million men of all nationalities and the average scores were calculated for each national group. For those little familiar with the English language, a separate test, the Beta test, was designed to obviate linguistic handicaps, the English-speaking recruits being given the so-called Army Alpha Test. The average scores of recruits from different countries were found to present rather wide variations. The records of a number of the leading national groups are given in the table (Table 30). Recruits from the nations of northern and western Europe (English, Scotch, 
Scandinavians, and Germans) tested relatively high. Those from southern Europe tested relatively low. Do these different scores indicate differences in native intelligence? Even those who had most to do in devising the tests and in compiling the results have conceded that the scores are influenced to a considerable extent by environment and training. Most psychologists nowadays do not consider

Table 30.-Percentages of Grades A and B and D, D-, and E in the Army Mental Tests According to National Origin

\begin{tabular}{|c|c|c|}
\hline Country & Grades $A$ and $B$ & Grades D, D-, and E \\
\hline 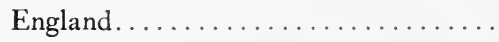 & 19.7 & 8.7 \\
\hline Scotland $\ldots \ldots \ldots \ldots \ldots \ldots$ & 13.0 & 13.6 \\
\hline 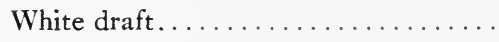 & I2. I & $24 \cdot 1$ \\
\hline Holland................ & 10.7 & 9.2 \\
\hline 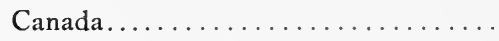 & 10.5 & $19 \cdot 5$ \\
\hline 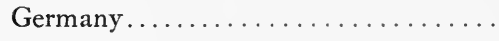 & $8 \cdot 3$ & 15.0 \\
\hline 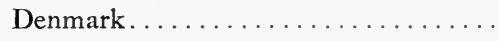 & $5 \cdot 4$ & $13 \cdot 4$ \\
\hline 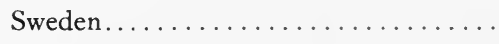 & $4 \cdot 3$ & I9. 4 \\
\hline 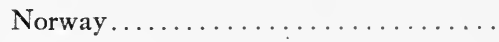 & $4 \cdot I$ & 25.6 \\
\hline Ireland. . . . . . . . . . . . . & $4 \cdot I$ & $39 \cdot 4$ \\
\hline All foreign countries............... & $4 \cdot 0$ & $45 \cdot 6$ \\
\hline 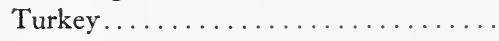 & $3 \cdot 4$ & 42.0 \\
\hline 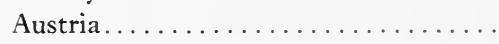 & $3 \cdot 4$ & $37 \cdot 5$ \\
\hline 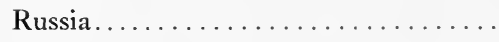 & 2.7 & 60.4 \\
\hline 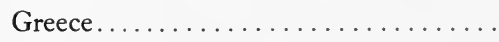 & $2 . I$ & 43.6 \\
\hline Italy $\ldots \ldots \ldots \ldots \ldots \ldots \ldots \ldots$ & 0.8 & $63 \cdot 4$ \\
\hline Belgium $\ldots \ldots \ldots \ldots \ldots \ldots \ldots$ & 0.8 & 24.0 \\
\hline Poland.$\ldots \ldots \ldots \ldots \ldots \ldots \ldots$ & 0.5 & $69 \cdot 9$ \\
\hline
\end{tabular}

such results as giving an adequate measure of differences in genetic factors affecting mental development. Demonstrably, as we have before remarked, training influences in no small degree the ability to make a good score on any mental test yet devised. This is shown by the fact that the scores on the Beta test were found to improve with longer residence in this country. It is indicated also by the wide range of scores on Alpha tests in the native-born recruits from states that differ from each other in the degrees of average educa- 


\section{HUMAN GENETICS AND ITS SOCIAL IMPORT}

tion. Whether or not extrinsic factors account entirely for the differences in scores is not definitely established.

And even if genetic differences occur as are indicated by the test scores, it would not follow that the tests afford an adequate gauge of the average intelligence of the populations in the countries from which the representatives in the army were derived. As we have seen, our recent immigrants from the south of Europe include a large proportion of unskilled and illiterate laborers whose average I.Q. is probably below that of the general population of the country from which they came. The same is shown by the studies of Gamio to be true of Mexico. It is reasonable to conclude that the under man is represented among recent immigrants to a greater extent than among the literate and skilled incomers from northern Europe, and the differences shown in the table, therefore, may result in part from the kind of selection occurring in the various national groups.

However we may interpret the findings of the army mental tests, several investigations have shown that the average scores of children of different national groups in the schools give results which are roughly parallel to those derived from adults in the army. In a study by Hirsch on the intelligence of school children in four small mill towns of Massachusetts the average test scores of different types of tests showed the following gradations: Polish Jews, Swedes, English, Russian Jews, Germans, Americans, Lithuanians, Irish, British Canadians, Russians, Poles, Greeks, Italians, French Canadians, and Portuguese. Over a third of the Portuguese and French Canadians, and almost a third of the Italians and Greeks had an I.Q. of less than 80. Whatever handicaps were caused by foreign languages they did not prevent high scores being made by the Polish Jews, Swedes, Russian Jews, and Germans. Kirkpatrick's studies on the I.Q.'s of children in Massachusetts gave averages on the 
Beta test of $\mathrm{IO}_{4}$ for Americans, IOO for Finnish, 83 for Italians, and 100 for one French Canadian school and 74 for another. Bere found in comparing the Stanford Binet Test scores of Italians, Bohemians, and Hebrew children in New York City that the averages were 85,93 , and 98 respectively. On the Pintner-Paterson Performance Test, however, the highest grades were made by the Italians and the Bohemians, the Hebrews falling considerably behind, whereas they were conspicuously in advance on the verbal tests.

Without going into details concerning the several studies on the relation of intelligence and nationality in schools, we may say that in general higher scores are made by native American children and children of northern European and Jewish ancestry, than are made by children of southern European, Mexican, Indian, and Negro ancestry. To how great an extent these differences are genetic and how far they are environmental in origin we have no clean cut and decisive evidence. Certainly environmental factors play a part. Probably occupational selection also plays a part. That genetic differences are also involved is a probability whose degree will be estimated differently according to one's familiarity with genetic principles, personal experience with the ethnic groups, and various other factors by which judgments in such matters are commonly influenced.

As might be anticipated the percentage of both mental defectives and of intellectually superior individuals varies with the average I.Q.'s as indicated by mental tests. The mental deficiency rates in the army were found to be high for Poles (5.8), Italians (5.5), Russians (7.4), AustroHungarians (6), and less for Canadians (2.4), Scandinavians (I.3), British (0.9), and Swiss (0.9). Even if the mental differences revealed by intelligence tests are not genetic they persist for at least two generations and probably more. They are in any case, therefore, of very great importance in 


\section{HUMAN GENETICS AND ITS SOCIAL IMPORT}

relation to the practical problem of regulating immigration. Granting that a stock with inferior intelligence will ultimately recover greatly from its handicaps, its presence in large numbers may seriously affect the welfare of a nation for a great many years. In crime certain foreign stocks contribute much more than their quota, or proportion to the general population. The quota for the Balkans, according to the studies of Laughlin, was 278 ; for Italy 218 , and for Russia 126, as compared with 82 for native Americans. According to the same investigator the foreign born contribute more than their quotas of insanity, epilepsy, and pauperism. That the native born contribute more than their quota of feeble-mindedness may be due to the fact that more cases of feeble-mindedness than of insanity and epilepsy are detected and not allowed to enter the country. The high ratio of the foreign born among the insane, etc., is probably due largely to their higher age composition, although their relatively low educational and cultural status is probably a contributory factor.

As a rule the crime records of southern Europeans have been high, while those of the foreign born from northern Europe have been below the average of the native-born white Americans. Records for homicide have been especially high for southern Italians, Mexicans, and Negroes. There can be no question that whatever advantages have been derived from the enormous immigration of the pre-war period it has directly or indirectly been productive of a number of drawbacks. How many generations will be required to overcome the disadvantages resulting from the presence of some of our immigrant stocks no one can tell. To the extent that these disadvantages result from hereditary deficiency they will remain indefinitely. There is much fine hereditary material in practically all the immigrant stocks we receive. The question of paramount importance from the 
biological standpoint is how the average hereditary qualities of our immigrants compare with those of our native population. Immigration may prove to be an important factor in determining the genetic quality of a people. Hitherto it has been treated as a purely economic matter, as if material wealth were more important than human beings. But, as was well stated by Dr. Laughlin, "immigration is a long-time investment in family stocks rather than a short-time investment in productive labor."

It has been urged that we are not justified in excluding any people on eugenic grounds unless it is definitely proved that they are of inferior hereditary quality. Granting that the low mental ratings of certain peoples are not definitely proved to rest upon a genetic basis, so long as the question is an open one why should an immigrant-receiving country assume the burden of proof? It might well be contended that the wiser course would be to shift the burden of proof to the other side and refuse to admit all aliens until it was established that their genetic endowments are not inferior to those of our own population. So long as action is called for, it is best to be on the safe side. Whatever its racial effects may have been in the past immigration can be so regulated as to be made an important factor in race improvement. By requiring high standards of admission from every country so that only those of sound physique and demonstrably good mentality and character are allowed to enter, the American people would not only be improved as to their hereditary qualities, but they would gain a population which would soon prove to be a valuable asset in many ways. At least until we begin to suffer from the ills of overpopulation we should adopt an immigration policy which would bring us only those who would raise the general level of our own inhabitants. There is no reason why this cannot be done. It would be simply political stupidity not 


\section{$34^{8}$ HUMAN GENETICS AND ITS SOCIAL IMPORT}

to avail ourselves of the opportunity. But there is always danger that, instead, the control of immigration will be exercised by interests caring nothing about the quality of the future population and bent only on securing the profits to be derived from cheap labor. Eternal vigilance may be required not only to preserve liberty but to prevent the best interests of future generations from being sacrificed on the altar of Mammon.

\section{Suggested Readings}

East ('27), chap. I4. Brigham ('23), ('30). Fairchild ('26). Grant ('34). Lorimer and Osborn ('34), chaps. 6 and 7. Kirkpatrick ('26). Thompson ('35), chap. 24. Taylor ('28-'30).

\section{Questions}

I. What relation is there between the birth rate and emigration in England, Norway, Hungary, Italy, and France?

2. Why does Italy now discourage emigration and why does Canada encourage it?

3. How does Australia control immigration? In what respects has her immigration policy been superior to that of the United States?

4. Compare the immigrants into the United States in I9I 3 with those in 1880 as to numbers, national origin, percentage of illiteracy, relative numbers of skilled and unskilled workers.

5. Does the literacy test make for the improvement of the eugenic quality of our immigrants? Give reasons.

6. In your private opinion are there any stocks in the United States, and if so which ones, that are undesirable additions to our population?

7 Why did Malthus think that emigration affords no permanent relief from overpopulation?

8. What diverse interests have figured in the regulation of immigration into the United States?

9. What influence has Mexican immigration exerted on the migration of southern Negroes into the Northern States?

I0. How has migration been provocative of race friction? Give illustrations from different parts of the globe.

II. Why has the rate of increase of the population of the United States been greater during the first decades of the nineteenth century than it was during the later decades? 


\section{CHAPTER XXIV}

\section{INBREEDING AND CROSSBREEDING}

UMAN beings have followed many customs in regulat1 ing matings on the basis of blood relationship. Frequently marriages are prohibited between near relatives and sometimes between those distantly related. On the other hand, the closest kind of inbreeding,- - that is, the marriage of brother and sister-has sometimes been practiced, as among the rulers of ancient Egypt and the Incas of Peru. The reasons which have led to the diverse and often fantastic marriage customs among the races of mankind concern the student of cultural anthropology rather than the biologist. We may safely conclude that marriages of near kin were not prohibited because of observations on their bad biological effects. Sometimes, to be sure, their biological effects are bad; but it is not likely that uncultured peoples with no knowledge of heredity would have found it out.

The real influence of consanguinity in marriage has been a matter upon which different views have been held even by special students of heredity. But since the wide applicability of Mendel's law has been established, geneticists are now practically agreed as to the interpretation of the results. The effects of inbreeding and crossbreeding in plants and animals are varied. Sometimes close inbreeding leads to a deterioration of the stock and sometimes it does not. Where deterioration occurs it commonly reaches a limit in a few generations after which it may be continued for a long period without further change. Inbreeding has the effect of bringing 


\section{$35^{\circ}$ HUMAN GENETICS AND ITS SOCIAL IMPORT}

out recessive defects which may be present in a stock and thus producing offspring inferior to the parents. If the parents are free from recessive defects, inbreeding produces no bad results.

Among human beings also close inbreeding is sometimes unfortunate and sometimes not, depending upon whether the parties are free from the same types of recessive defects. The marriage of Charles Darwin and his first cousin produced four distinguished sons; and many other cousin marriages have likewise proved to be very fortunate in their results. Many of our states prohibit cousin marriages and Oklahoma even prohibits the marriage of second cousins; but it is doubtful if such laws are biologically defensible. Some cousin marriages should be encouraged and others should be discouraged on biological grounds. Whether or not such marriages should be contracted depends upon the heredity of the parties concerned.

The biological effects of crossing the different races of man is a subject upon which opposed views still prevail. With groups which differ so little as the racial stocks of Europeans, there is no convincing evidence of any bad effects resulting from intermarriage. The people of most European nations are notoriously of mixed racial origin. Despite the many advocates on both sides of this question there seems to be no clear indication that people who are relatively pure racially are either superior or inferior to others of highly mixed composition. Much information has been collected concerning the way in which certain anthropological features characteristic of races are inherited in the hybrids. Some characters such as eye color behave as simple Mendelian units, while others such as stature, size, and skin color are dependent upon multiple factors and appear to be blended in the progeny. But color of eyes and hair, head form, thickness of lips, high cheek bones, shape and size of 
the nose, and many other characteristics employed to distinguish races have, so far as is known, very little significance from the standpoint of biological fitness. They may be significant aesthetically, depending upon the particular taste of the individuals interested, and hence may be affected by sexual selection, but it is difficult to see how, per se, they could have much relation to survival.

The question of chief interest in relation to race crossing is whether, as has often been alleged, the mingling of very different races is productive of inferior progeny. Such crosses should be judged in the light of both their biological and their social effects. From the purely social point of view interracial marriages are apt to be unfortunate because of the very different traditions, customs, and mental attitudes of the two races. It frequently happens that the half-breed is a person of uncertain social status, and he may suffer some degree of demoralization on this account. The status of racial hybrids varies greatly in different regions. In most parts of South America it is no drawback to be a mongrel. In other parts of the world the psychological attitudes of the people make the position of the cross-breed very undesirable and cause much unhappiness in those who venture to marry outside of their race.

In dealing with the purely biological aspects of race crossing we should consider two problems: (I) the possible disharmonies of constitution that might result, and (2) the effects of crossing superior and inferior races, provided, of course, any one race is superior or inferior to any other. In regard to the first of these problems it seems probable, $a$ priori, that, on account of Mendelian segregation in the second and subsequent generations, the mingling of very distinct races would result in many inharmonious combinations. Each race may be assumed to have evolved in the course of time a fairly well adjusted combination of organs. 


\section{HUMAN GENETICS AND ITS SOCIAL IMPORT}

If the members are tall or short, the proportions between the lengths of legs, arms, breadths of shoulders, and sizes of other parts would be much the same in each race. In so far as these traits are inherited as Mendelian units, one might find in the second generation combinations of long legs and short arms, or various other associations of parts which would detract from the general effectiveness of the whole. As Davenport has pointed out, such combinations as large teeth in small jaws are not uncommon, as well as the reverse combination of large jaws and small separated teeth. "Nothing is more striking," says Davenport, "than the regular dental arcades commonly seen in the skulls of inbred native races and the irregular dentitions of many children of the tremendously hybridized Americans." In Davenport's opinion, "A hybrid people are a badly put together people." Perhaps they are apt to be a homely people, for beauty implies a certain proportionate development of parts, and such combinations as large heads and small jaws or the reverse, or a disproportion between the size of the nose and the rest of the countenance are not conducive to facial beauty. According to Dr. Mjoen the crosses between very different racial stocks, such as the Swedes and the Lapps, are characterized by an unusual number of anatomical disharmonies. On the other hand, the distinguished geneticist, Dr. W. E. Castle, believes that there is little evidence of disharmonies resulting from race crossing, at least where the races are not widely dissimilar. In his experiments on crossing large with small breeds of rabbits, Castle finds that the progeny are intermediate in size and retain their intermediate size in subsequent generations. Moreover, they are vigorous, prolific, and show no indications of having incongruous jumbles of parts. One reason for this is probably the fact that size depends upon many Mendelian factors. Also the size of a particular organ is to a large extent determined 
during development by its physiological relationships to the whole organism. The influence of functional balancing and adjustment would doubtless tend, in a measure at least, to overcome any disharmonies resulting from Mendelian segregation. Nevertheless, the fact that Mendelian segregation is expressed somatically at all would seem to make it very probable that it would sometimes lead to unfavorable combinations of characteristics. This is indicated by the results of some wide crosses between plants in which the second generation of hybrids consists practically of only two types closely resembling the original forms that were crossed. Out of the many combinations produced, apparently only those closely resembling the two grandparents are able to survive. In such crosses intermediate types do not persist, and there is a reversion to the parental species. While there seem to be cases of rather incongrous aggregates of parts in many crosses between distinct races of men, we have as yet little definite information concerning the extent to which such crosses are productive of deterioration either physical or mental. One can only say that there is a distinct danger that undesirable effects may follow from the crossing of remote races.

If one race is superior physically or mentally to another, it would doubtless be better for the more highly endowed race to preserve its own inheritance rather than to dilute it by miscegenation. There are many who contend that, barring possible minor differences of temperament or special aptitudes, all races are about on the same level of mental development. Formerly backwardness in cultural achievements was frequently regarded as indicative of deficient mental ability, and, as a result of this conclusion, the native intelligence of primitive peoples was greatly underrated. This fact is brought out clearly in the well-known book of Dr. Boas on The Mind of Primitive Man. Later studies on the intelligence 


\section{HUMAN GENETICS AND ITS SOCIAL IMPORT}

of primitive peoples have been more critical than the rather loose observations and deductions of the older anthropologists. When mental tests came to be applied, it was found that different races made different average scores. Chinese and Japanese children in the United States and Hawaii, when given tests in which language handicaps are minimized, have usually made very good records. The studies of Garth on I, 050 full-blooded American Indians gave an average I.Q. of only 68.6. Also, I,004 Mexicans and I,272 Texas Negroes tested by the same investigator made average scores of 78 and 75 respectively. Jamieson and Sandiford found that Canadian Indians gave a score of 97 on the Pintner NonLanguage Test, $9^{2}$ on the Pintner-Paterson Performance Test and 80 on the National Intelligence Test. According to Garth, the test scores of mixed bloods are higher than those of pure-blood Indians, and several investigators have reported that Negroes make higher scores in proportion as they are light in color. The results of mental tests in Negroes vary widely according to geographical area and the associated differences in educational opportunities. The same is true, as we have pointed out, for the whites. Unquestionably, the low scores of races with inferior cultural status are at least in part due to educational handicaps. Whether, as is maintained by a number of anthropologists and psychologists, all mental differences can be thus accounted for is a question which the mental tests alone do not enable us to solve.

It is a fact of much interest in relation to this problem that, if one may gauge intelligence on the basis of achievement, the mental development of mulattoes is superior to that of the pure blacks. As Reuter has shown, the intellectual leaders among the Negroes show a very decided preponderance of individuals with white blood. College graduates, doctors, lawyers and writers of Negro extraction are mostly mulattoes. 
Of I 43 life members of the National Business League I 4 were black, 60 were dark or medium colored mulattoes, while 7I were light mulattoes or near-whites. Reuter has attempted to explain the striking superiority of the mulattoes in all intellectual achievements partly on the grounds that the mulattoes enjoy a superior culture to the blacks, and partly because in the period of slavery "the choicest females of the black group became the mothers of a race of half-breeds." How far the intellectual superiority of female slaves increased their chances of bearing children to white fathers is quite uncertain. After emancipation race mixture probably occurred most frequently between the inferior members of both races. The process of social selection may have favored the mulattoes more than the pure blacks, but the extent of this influence is a matter of conjecture. According to many other students of the problem the most natural and reasonable explanation of the superior achievements of the mulatto is that they are due to the infusion of white blood.

In general we may say that the proper evaluation of the native abilities of the different races of man presents formidable difficulties, and that our knowledge concerning it is in a most unsatisfactory state. This is especially unfortunate because the subject is an important one. For the proper regulation of immigration and the determination of policies of population control, to say nothing of legislation on interracial marriage, such knowledge would be of much value. But in view of the uncertainties of our knowledge it may be urged that for a race which has demonstrated its capacity for superior intellectual achievements it would be the part of prudence to avoid amalgamation with other races concerning whose genetic worth we do not have adequate information. Ignorance is never a sufficient justification for taking a leap in the dark. And until more scientific knowledge is obtained on this highly controversial subject, for racial 


\section{HUMAN GENETICS AND ITS SOCIAL IMPORT}

stocks which are now in the van, race mixture may be regarded as a dangerous experiment.

The mingling of races goes on speedily enough in any case. Owing to increased facilities for travel, peoples are coming to be mingled together over extensive regions of the earth. In its early stages the human race as it spread over the surface of the globe became differentiated along many different lines. Isolation favored divergence. But now barriers are being broken down. The development of industry has greatly fostered migration, and the amalgamation of races is proceeding at an unprecedented pace. The period of increasing differentiation is being followed by a period in which differences are becoming merged in a common heterogeneous aggregate. Will the process of fusion ultimately make all races one? There are those who welcome such a consummation as affording a final solution to all embarrassing race problems and helping to usher in a reign of peace and universal good will. But how would it affect progress, biological and social?

It should be pointed out that the biological effects of race mixture depend to a large extent upon how the process goes on. Where it results in the free intermarriage of the two races it tends to produce an intermediate though highly heterozygous stock. If, on the other hand, it results from the illegitimate unions of the males of one race and the females of the other, it is mainly at the expense of the race that furnishes the females. In illegitimate unions between white men and Negro women the women are prevented for some time from bearing children to black fathers, while the white men are not prevented from producing children by white mothers. Inasmuch as in the United States most children resulting from crosses between the two races come from the mating of black women and white men, and only very rarely the reverse, race assimilation becomes largely a one-sided process 
in which white germ plasm is substituted for black in the offspring. One may say that white genes are infused into the Negro race, but that fewer black genes enter the white race. The Negroes, therefore, are becoming bleached, whereas the whites are not correspondingly blackened. When the Negroes have a sufficient ingredient of white blood and for this reason are no longer prevented from marrying whites, the black genes may then become slowly disseminated in the white race. But until this occurs race assimilation will be largely at the expense of the Negro. Many light-colored mulattoes "pass" as whites. The extent to which persons with some Negro blood succeed in marrying whites is open to much doubt. Even laws as drastic as that passed in Virginia which forbids marriages between whites and persons having the least trace of Negro blood cannot prevent the slow infiltration of Negro genes into the white race. This process may continue until it leads to complete amalgamation, but if the Negroes are destined eventually to be absorbed by the whites they will be considerably bleached before they are assimilated.

\section{Suggested Readings}

Castle ('26 and '30). Davenport ('II), chap. 5. ('I $3 a$ and b). East and Jones ('I 8). Holmes ('2I), chap. II. Ludovoci ('33). Mjoen ('26). Reuter ('18, '31).

\section{Questions}

I. Why is deterioration through inbreeding very marked in some lines and slight or nil in others?

2. Why does deterioration through inbreeding proceed with relative rapidity in the first few generations and then more slowly until it practically ceases to occur?

3. If the marriage of first cousins produced an albino child, what would be the chance that the next child would be an albino?

4. Since intreeding brings out recessive defects would it be desirable to encourage it in order that the genes for such traits be made manifest and caused to disappear? 


\section{$35^{8}$ HUMAN GENETICS AND ITS SOCIAL IMPORT}

5. In the pedigree of Otto and Ludwig of Bavaria (Fig. 55) what individuals are probably heterozygous for dementia praecox?

6. How do laws regulating racial intermarriage vary in different states?

7. What efforts are being made in Germany to regulate intermarriage between different racial stocks?

8. What do you consider the more important, the social or the biological consequences of racial intermarriage?

9. Why do you think that the proportion of mulattoes to blacks in the United States increased after emancipation and then decreased?

ro. Since miscegenation still continues, even if on a reduced scale, will it eventually lead to the complete fusion of whites and Negroes?

Ir. What legal restrictions, if any, should be imposed on consanguineous marriages? What laws on the subject are there in your own state?

12. What is the prevailing attitude on race mixture in South America, in most English-speaking countries, in most Mohammedan countries?

13. Do you think race mixture will proceed more rapidly as time goes on? 


\section{CHAPTER XXV}

\section{PROPOSED MEASURES FOR RACE BETTERMENT}

WE ARE naturally prone to look upon evolution as $\checkmark$ having reached, at least approximately, its final stage in our present human species. But if we view the matter from the vast perspective of geological time, it will seem highly improbable that man will remain indefinitely in the position he has now attained. If we could look upon our descendants fifty or one hundred million years hence, provided that any of them will be left, we might not recognize them as even remotely our own kin. We may amuse ourselves by speculating as to what sort of creatures they might be, but our vision into the near future is obscure enough; the remote future is enshrouded in complete darkness.

Man's evolution is to a certain extent under his own control. As horses can be bred for the racecourse or for heavy draft animals, so human beings can be caused to develop in this way or that according to the kind of selection exercised in perpetuating the race. The control of human biological evolution is the concern of practical eugenics, as distinguished from theoretical eugenics, which deals with human heredity and the causes by which it is changed. The notion that human beings, like domestic animals, may be improved by selective breeding never attained wide currency until recent years, if indeed it can be said to have done so now. One of the earliest advocates of practical eugenics was the Greek poet, Theognis, who lived in the sixth century в.c. In one of his poems he says:

With kine and horses, Kurnus, we proceed

By reasonable rules, and choose a breed 
For profit and increase, at any price, Of a sound stock without defect or vice. But in the daily matches that we make The price is everything; for money's sake, Men marry; women are in marriage given. The churl or ruffian, that in wealth has thriven, May match his offspring with the proudest race. Thus everything is mixed, noble and base.

If, then, in outward manner, form and mind, You find us a degraded, motley kind,

Wonder no more, my friend; the cause is plain, And to lament the consequence is vain.

Among the Spartans there were several practices aimed at creating a strong, brave, and healthy race. According to Lycurgus only the best men should be permitted to beget children. Ages at marriage were regulated by the theory that children procreated when the parents were in their period of greatest vigor would be exceptionally strong and healthy. Newborn children were submitted to an inspector who, if they were sound and healthy, decreed that they be brought up. If they were weak or puny, they were not allowed to live. Lycurgus taught that children belonged to the state and not to the individual. And parental affection was not suffered to foster children who would probably develop into weak or unhealthy persons.

The Greeks in general recognized the value of good heredity. "The fairest gift that one can give children," says Heraclitus, "is to be born of noble sires." The practice of "exposing" weak and deformed infants was common among the Greeks and also the Romans and was advocated by both Plato and Aristotle. Plato, who elaborated the most thoroughgoing scheme of eugenics ever devised, would place the determination as to who should produce offspring and the number of individuals composing the city state in the hands of the rulers. In the ideal republic ample liberty of begetting off- 
spring was to be granted to the youth who distinguished themselves in war or otherwise, and the custodians to whom the children were committed should see that they received the best nurture, "but the children of the more depraved and such others as are in any way imperfect they will hide in some secret and obscure place." The governors were to determine the number of marriages contracted, and it was recommended that "an ingenious system of lots should be made, that the inferior man may accuse his fortune and not the governors of the manner in which the couples are joined." In short, the whole matter of perpetuating the race, both as to numbers and quality, was to be managed by the governors, even though they found it necessary to resort to a little chicanery to accomplish their worthy ends.

If romantic love and parental affection were given no weight, and human beings were bred like so many cattle, the race could doubtless be greatly improved in quality in a few generations. Plato's scheme of eugenics, like so many other measures advocated in the Republic, was never regarded as feasible of adoption, and it has remained as a philosopher's ideal, commendable in theory, but unsuitable for practical application.

The eugenic proposals of Aristotle were much more modest. He recommended that the husband should be twenty years older than his wife, and that offspring should not be begotten by men in their old age. $\mathrm{He}$ also held that the population should be limited by restricting the period of propagation, or, if this failed, through the employment of abortion. He recognized the importance of superior parentage, and counseled that "nothing imperfect or maimed be allowed to grow up." Aristotle had no systematic plan for racial improvement. His measures were negative and restrictive rather than aimed at continuous race betterment. 


\section{HUMAN GENETICS AND ITS SOCIAL IMPORT}

Beyond being exercised over the fall of the birth rate, the Romans had little notion of eugenics. Neither did this subject receive consideration during many centuries of the Christian era. In 1683 Campanella in his City of the Sun pictured an ideal society in which reproduction is not left to the inclinations of the individual, but is regulated by the state in the interests of posterity, more or less after the fashion of Plato's Republic. After this scarcely any thoughts on eugenics came to expression until well along in the nineteenth century.

The influence primarily responsible for the modern eugenics movement was the establishment of the doctrine of organic evolution following the publication of Darwin's Origin of Species in I859. The great intellectual revolution which resulted has profoundly affected all the sciences dealing with man. Naturally, if man was evolved from some lower form of animal life and is subject to the same biological laws as the rest of the organic world, he must be capable of further evolutionary changes. Darwin's cousin, Francis Galton, who is universally recognized as the founder of modern eugenics and to whom the name eugenics owes its origin, was greatly stimulated by the ideas set forth in the Origin of Species. His early work on Hereditary Genius, in which he brought together many evidences that human beings differ greatly in their inherited endowments of brains, inevitably implied the possibility of raising the mental level of the race by selective breeding. The idea of race betterment formed the mainspring of much of Galton's work in heredity, biometry, psychology, and anthropology. His books on Natural Inheritance, Inquiries into Human Faculty, Noteworthy Families, English Men of Science, Essays on Eugenics, and many articles and addresses show that his life work was mainly devoted to supplying a scientific basis for race improvement. In $190_{4}$ he founded a research fellowship, and in 1905 a scholarship in 
eugenics in the University of London, and at his death his fortune was bequeathed for the establishment of a professorship of eugenics in the same institution. Largely as a result of his influence, the Eugenics Education Society was founded in I908. This society has been active in stimulating eugenic research and in spreading the knowledge of eugenics through

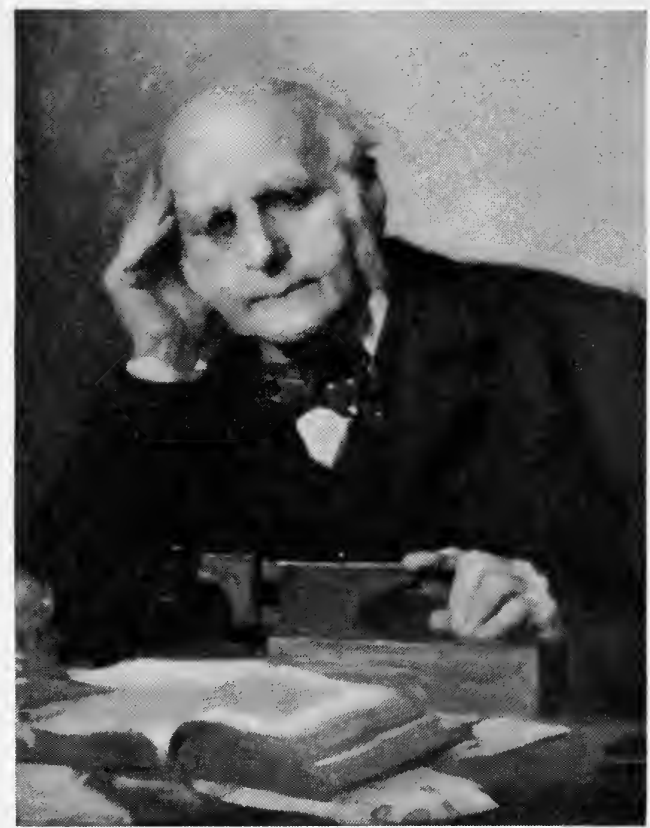

F1G. 83.-Sir Francis Galton. (From Galton, Memoirs of My Life, Methuen and Company.)

its official organ, The Eugenics Review. The Galton Laboratory has been very productive in researches in human heredity and eugenics. The first to occupy the professorship founded by Galton was the noted mathematician, Professor Karl Pearson who has developed many of the mathematical methods and formulas used by workers in biometry. The Galton Laboratory issues several publications, the Memoirs, the Lecture Series, and more recently the Annals of Eugenics, which contains more extended and technical contributions 


\section{HUMAN GENETICS AND ITS SOCIAL IMPORT}

than those appearing in The Eugenics Review. The publications of the Galton Laboratory have attracted widespread attention and have materially advanced the eugenics movement.

In the United States the Eugenics Record Office, located at Cold Spring Harbor, Long Island, N. Y., is more or less

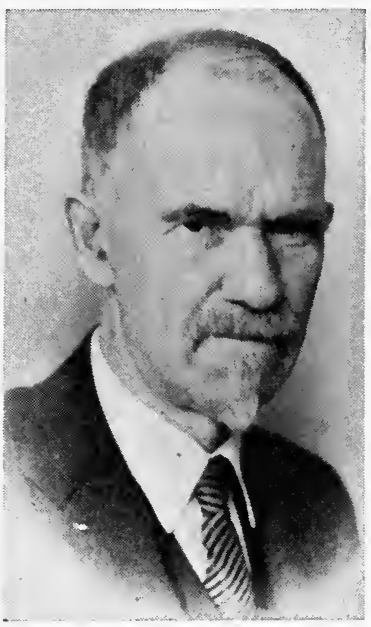

FIG. 84.-C. B. Davenport. (From a photograph loaned by $\mathrm{Mr}$. Trevor Teele.) comparable to the Galton Laboratory. It issues a series of Memoirs and Bulletins on human heredity and eugenics, and its Director, Dr. C. B. Davenport, has been a very active and productive worker in this field. The Eugenics Record Office employs a number of field workers who have made many investigations of family pedigrees especially in New England and adjacent states. Eugenics societies have been formed in many countries, Norway, Sweden, France, Holland, Austria, Japan, Italy, Russia, and especially Germany where there is now an active and widespread interest in eugenic reform.

The number of periodicals devoted wholly or in part to this subject has greatly increased, and discussions of eugenic problems now find their way much more frequently into many other journals both scientific and popular. Courses on eugenics are given in many colleges, and the subject receives attention in many courses on biology, psychology, and the social sciences. Most of the societies concerned with eugenics perform a twofold function of promoting research and disseminating knowledge of the principles of the subject. There is a growing appreciation of the role of heredity in several fields in which this factor was formerly almost completely 
ignored. Students of population problems are now concerned not only with the quantitative aspects of the subject, but with the question of quality as well. This is shown in the various conferences on population held in recent years, and also by the contents of the journals dealing with population problems.

One of the most important population problems is presented by the birth control movement, since this affects not only the.quantity but also the quality of the population. At its inception this movement was motivated largely by the humanitarian desire to relieve parents among the wage earners from the burden of large families. It was only too apparent that the large families in the lower economic classes entailed much hardship on parents and offspring alike. The earlier proponents of birth control, R. D. Owen, Francis Place, Richard Carlile, Charles Bradlaugh, Mrs. Anne Besant, and J. S. Mill, were persuaded that the voluntary limitation of offspring affords the only feasible means by which the wage earners can secure a fair standard of living and give their children the advantages needed for their proper development. Birth control, however, made its most rapid progress in the wealthier and more cultured members of society. It is therefore responsible for a large part of the differential birth rate about which the eugenists complain.

On the other hand, the advocates of birth control contend that it is their aim to spread the knowledge of contraception through the lower strata and thus bring about an equalization of the birth rates, as has, in fact, been done to a large extent in a number of localities. It is useless to try to induce the upper strata to outdo the proletariat in rapidity of childbearing. The only remedy for the dysgenic effects of birth control, it is claimed, is more birth control. Besides affording a protection against the evils of overpopulation and doing 


\section{HUMAN GENETICS AND ITS SOCIAL.IMPOR'T}

away with much misery and hardship resulting from large families supported on small incomes, birth control, it is contended, really affords the most feasible method of eugenic reform. Among eugenists in general there has been a gradual change of attitude toward the subject from one of open hostility, or at least suspicion, to one of qualified approval or even ardent advocacy. Most eugenists realize that, as Mr. Wiggam remarks, "birth control is a two-edged sword." Whether its effects are eugenic or dysgenic depends upon how it is employed.

Eugenic procedures may be grouped into two classes: Those whose aim is to reduce bad heredity come under the head of negative eugenics; those intended to increase good heredity are classed under positive eugenics. This division is based upon judgments of value, and hence it is possible that there might be differences of opinion over what kinds of heredity are good or bad. Similar differences sometimes arise as to the evaluation of conduct as good or bad, but there is in both cases a sufficiently broad basis of agreement for many, if not most, practical purposes. Nearly all sensible people would consider it very desirable for the race to get rid of the genes responsible for hereditary insanity, epilepsy, feeble-mindedness, blindness, deafness, split hands, and other gross or incapacitating defects. Most people would agree also as to the desirability of having more of the kinds of heredity which make for sound physical development and superior intelligence. There is no need to arrive at a general consensus of opinion as to just what traits individuals should or should not possess. In fact, it is highly desirable that a people should contain a high degree of genetic diversity. A good deal of criticism has been directed against eugenists on the assumption that they propose to inaugurate a detailed scheme of arbitrarily regulated matings in order to produce their own favorite brand of human beings. But this kind of 
eugenist exists mainly in the imagination of the critics. Beyond curtailing the procreation of persons with obviously undesirable defects no one, except a few negligible extremists, advocates any arbitrary interference with the propagation of any kinds of hereditary traits.

Misconceptions of eugenics readily become very prevalent because most people who are otherwise well educated are woefully ignorant of the rudiments of biological knowledge, and especially knowledge of genetics. Even cultivated people sometimes ask in all seriousness: "Do you really believe in heredity?" That the important function of perpetuating life should be carried on in almost complete disregard of whether the kind of life that is perpetuated will improve or deteriorate the race is especially unfortunate when people are taking the regulation of the birth supply more and more into their own hands. Among our remote ancestors life was transmitted solely in obedience to an instinctive urge, and natural selection took care of the failures. Now that procreation has come to be largely a matter of voluntary choice and since so much is done to preserve the unfit, it becomes increasingly important that the perpetuation of life should be carried on intelligently and with due regard to the welfare of future generations.

In considering proposals for eugenic reform in the fields of both positive and negative eugenics we must assign a position of prime importance to eugenic education. If any eugenic reform is ever to be achieved in a democratically governed country, eugenic education is an indispensable prerequisite. Measures for eliminating bad germ plasm and schemes for increasing the birth rate among those with superior heredity must at least meet with popular approval before they can become effective, and popular approval will not be forthcoming for any really adequate eugenic system in the present condition of unenlightenment. 


\section{HUMAN GENETICS AND ITS SOCIAL IMPORT}

Many of the methods coming under the head of negative eugenics have to do with the regulation of marriage. From the biological standpoint a large part of our legislation on marriage in the United States is peculiarly unintelligent. Laws regulating consanguineous unions are mostly useless. Many states still permit the marriage of boys of fourteen and girls of twelve years of age. The stocks in which mere children marry are generally of inferior status and are likely to be of poor quality. When the reproductive career is begun at an early age two or three children are apt to be born before most families get started. Both eugenically and socially, child marriages are very undesirable and should be prohibited. Regulations designed to restrict rash and illadvised marriages would probably have a eugenic effect in addition to being desirable on other grounds. Marriages contracted after a few hours' acquaintance are apt to prove disillusioning to the impetuous contracting parties. According to Popenoe and Johnson, "In Los Angeles County alone ... more than a thousand couples each year go to the courthouse and apply for a license to wed, and then do not come back three days later to get it. What happens to these abandoned romances would make an interesting investigation. While the facts often cannot be obtained, indications suggest that a large proportion of them represent freak marriages, fraudulent marriages, drunken marriages, runaway marriages, and others whose consummation could have been of no value to society."

Laws requiring a medical examination before marriage are sometimes described as eugenic, but they are more properly hygienic as their main intent is to check the dissemination of venereal disease. Most states in which such laws have been passed require a medical examination only of the males. These laws may have a considerable value, although they do not always work out in the way that was intended. There 
are a few clergymen who refuse to perform a marriage ceremony unless the applicants can present a certificate of health. Where blindness, obvious mental deficiency, and many gross deformities or defects are transmitted by marriage, generation after generation, to the affliction of numerous descendants, one wonders with what conscience the clergymen, priests, or others empowered to unite individuals in the bonds of wedlock were able to perform the ceremony. A person who solemnizes the marriage of hereditary defectives is really a party to a criminal act, a crime against posterity that may result in untold evils for a long time to come. And yet such procedures go on apparently without eliciting many qualms of conscience on the part of the officiating individuals. Every day marriages take place which would not be tolerated in most tribes of savages.

All of our states have laws forbidding the marriage of idiots, imbeciles, and the insane, but these were passed not so much for eugenic reasons as because the individuals concerned were deemed incapable of making a legal contract. Very little care is exercised in issuing marriage licenses, and if an individual is refused a license in one county he can generally get it in another. As a matter of fact, these laws have little effect in preventing marriages that should not occur. With the feeble-minded the prohibition of marriage is apt to be inadequate as a means of preventing reproduction, as is indicated by the very high proportion of illegitimate children in mentally defective stocks.

It is generally recognized that there are many defective individuals who in the interests of posterity should not be permitted to reproduce their kind. The two methods most commonly employed to prevent the multiplication of such persons are segregation and sterilization. Segregation is usually employed for other than eugenic reasons; the prevention of reproduction during the periods of isolation is only 
an incidental result. Of the eugenic effects of the segregation of criminals we know little; but for social reasons, if for no others, it is doubtless desirable that criminals produce few children. Most of the insane are confined either because they are dangerous when at large or because they can be cared for much better in special institutions for the purpose than in private homes. In the United States there are roughly about 300,000 insane in hospitals, and there is probably an equal or greater number outside of hospitals. Discharges are frequent, and it is estimated that about three out of every four persons discharged as cured are apt to be committed again at some future date. Many of the insane have had children before their commitment, and children are begotten by others after their discharge or during periods of parole. Institutional segregation doubtless interferes with the reproduction of those suffering from mental disease, although it is far from an adequate preventive under present conditions of parole and limited periods of detention.

There are fewer feeble-minded than mentally diseased persons in institutions, but a larger proportion of them are unconfined. On the average the number segregated in the United States is about 60,000. The White House Conference of I930 estimated that approximately 2 per cent of the population is definitely feeble-minded-which means that in the United States about two and one-half millions would fall in this category. If this be true, the statement of E. R. Johnson, the Superintendent of a large institution in New Jersey, that no state provides facilities for the care of more than Io per cent of its mental defectives is well within the facts. Many of the higher grades of mental defectives can be made self-supporting, but the lower levels are entirely a burden. The total cost, direct and indirect, of mental deficiency is enormous. The segregation of all of the feebleminded would entail an expense that most communities 
could not be induced to defray. Yet the cost of their upkeep has somehow to be met. Evidently, so long as the great majority of the feeble-minded remain at large, the problem of preventing their reproduction is not solved. From the very magnitude of the undertaking segregation is failing to supply an adequate remedy.

The alternative proposal that is widely advocated is sterilization. In the male this is a slight operation consisting in severing the vas deferens of each testis and thus preventing the escape of spermatozoa. The operation commonly adopted for sterilizing females involves cutting the oviducts, or Fallopian tubes, thereby preventing the ova from gaining access to the uterus. Neither operation interferes with the sex functions and there are apparently no definite aftereffects upon general health or vigor. The chief and almost the only effect of the operation in both sexes is to render the individual sterile.

Laws authorizing the sterilization of defectives have been passed in twenty-eight states of the Union, and up to January I, I935, 20,063 legal sterilizations have been performed, 8,644 on males and II,4 19 on females. California has the distinction of having performed nearly as many operations $(9,93 \mathrm{I})$ as all the other states combined. In many states the passage of sterilization laws has been followed by practically no operations. Several of the sterilization laws have been declared unconstitutional or otherwise set aside by higher courts. The case of Buck vs. Bell, originally tried in Virginia, was finally carried to the U. S. Supreme Court which upheld the Virginia statute. In presenting the grounds for the decision, Justice O. W. Holmes made the remark that "three generations of imbeciles are enough."

There has been a good deal of opposition to sterilization on several grounds. Some have regarded it as an unwarranted infringement of personal liberty, and one which creates a 


\section{HUMAN GENETICS AND ITS SOCIAL IMPORT}

dangerous precedent. It has been criticized as inflicting "cruel and unusual punishment," and hence contrary to the Constitution. It has been opposed on the ground that we do not know that mental defects and diseases are transmitted by heredity, and hence we should not deprive anyone of the power of reproduction. There has been opposition on religious grounds, especially by adherents of the Roman Catholic Church, and it has been urged that by removing all danger of producing offspring sterilization would lead to an increase of sexual immorality and a greater dissemination of venereal disease. A priori this seems to be a probable conclusion; but like so many plausible deductions it is not borne out when subjected to the test of inductive investigation. The subsequent careers of many sterilized individuals in California have been followed by Dr. Paul Popenoe in the endeavor to ascertain to how great an extent sterilization may have acted as an incentive to irregular sex behavior. Of the sterilized feeble-minded males, "not a single case of the sort has arisen." This may be attributed to the fact that as a rule feeble-minded males are not given to sex offenses. Then among the sterilized insane "only one case was found where a patient tried to use the fact of his sterilization as an argument for promiscuity." On the other hand, the feeble-minded girl is characteristically prone to loose sexual relationships. About three-fourths of the girls in the Sonoma State Home in California were sexually delinquent before they were committed. After they had been sterilized and released on parole, "only one out of every twelve has been a sex offender." The follow-up study which included a canvass of probation and parole officers and social workers familiar with the history of the girls liberated on parole furnished only about a half-dozen cases in which it was alleged that sterilization may have afforded an inducement to sexual irregularity. In two of these instances it seemed clear that 
the offenses would have occurred anyway, but in any case the percentage of sexual delinquency attributable to sterilization is relatively very small, about one in a thousand.

It cannot be asserted that sterilization is of itself an agent of moral reformation. The reason why sterilization has been working so successfully in California is because it is combined with a policy of supervision after release. The sterilized girls are placed so far as possible in responsible families, and if their behavior is not satisfactory they may be sent back to the institution. One cannot, of course, expect ideal behavior in these girls, especially in the light of their previous history. Many of them marry, and a study of 125 such marriages showed that, on the whole, they have turned out as successfully as could reasonably be expected. Marriage acts as a stabilizing influence, and apparently contributes to the welfare of both parties. The fact that it results in no children lightens the economic burdens of the married couple and reduces somewhat the number of defective offspring. When we consider the strong tendency to assortative mating on the basis of levels of intelligence, it will not seem likely that the men who marry these girls would have contributed much to the improvement of our racial heredity. Hence the loss of their genes is not to be deplored.

In California most of the feeble-minded and a considerable proportion of the insane who have been committed to institutions are sterilized before they are released. The great majority of the persons sterilized either welcome the operation or make no objection to having it performed. Social workers, parole officers and others in close touch with the effects of the operation are, in general, strongly in favor of it. Sterilization is coming to be practiced in several foreign countries. The largest number of operations have been carried out in Germany under the recent sterilization law. During the first year of its operation sterilizations were 


\section{HUMAN GENETICS AND ITS SOCIAL IMPORT}

performed upon 56,244 persons adjudged to be hereditarily defective by the High Courts of Eugenics-over twice as many sterilizations in one year as the total number performed in the United States.

In no country do the number of individuals sterilized constitute more than a small fraction of the hereditary defectives who in the interests of the race should not procreate their kind. Even if all defectives were sterilized there would be a continuous supply from the reservoir of carriers. Opponents of sterilization have urged that if all of the feebleminded and insane were prevented from reproduction, it would make little difference with the number that would be produced in any one generation. This statement is commonly based on the assumptions that (I) mental defect is due to a single recessive gene, and (2) that the defect is recessive to the extent that heterozygous individuals appear normal. Both of these assumptions, as we have seen, are open to serious question. But, granting that they are both correct, it is evident that sterilization would cut off at least a certain proportion of our hereditary defectives. If a recessive trait is rare, the sterilization of those possessing it will effect its reduction with extreme slowness. If, however, the trait should occur in only I per cent of the population, the sterilization of all affected individuals would reduce it by 17.4 per cent in the next generation, and this reduction would be permanent. With further reduction the rate of elimination would be slower. The usual calculations of the rate at which recessive traits can be eliminated are based upon the further assumption that matings occur at random, and this assumption is never realized. There is, especially in mental defect, a strong tendency to assortative mating. Besides, for social and economic reasons, people of limited mentality often tend to segregate in certain areas. There, through a series of more or less closely inbred generations, recessive genes may 
accumulate and give rise to a plentiful crop of subnormal individuals, as is illustrated by the Jukes, Hill Folk, and other defective stocks. If all feeble-minded individuals of these stocks could have been sterilized, it would have made a very great difference in the number of such individuals born.

If one could eliminate all bad heredity from the human race, mankind would either become extinct or greatly improved in quality. According to Malthusian principles, if fewer people with bad heredity were born, people with good heredity would automatically respond by an increased birth rate. It is open to serious question, however, whether measures coming under the head of negative eugenics will be sufficient to prevent the race from being perpetuated mainly by people of subnormal quality. Certainly the segregation or sterilization of the small percentage of persons who are defective enough to warrant official commitment cannot be expected to contribute much toward this end. Negative eugenics based upon compulsory restrictions probably cannot be carried out on a very extensive scale. It has been contended that birth control in the borderline and dull-normal groups, who are usually on an economic status that would make small families desirable, would do much more. How much the extension of contraceptive information in these groups is likely to accomplish-and it has possibilities of doing a great deal-is a question involving all the uncertainties of predicting human behavior. But can any or all negative eugenic procedures result in rescuing from extinction the stocks that are now failing to perpetuate their kind? In the opinion of many eugenists the present tendency for success to be correlated with sterility can only be effectively counteracted by providing incentives that will lead to an increased birth rate of the better endowed elements of the population. Whether anything can be done in this direction 


\section{HUMAN GENETICS AND ITS SOCIAL IMPORT}

that is likely to be fraught with any considerable measure of success is a problem of the greatest importance, but it is one to which the general public shows little disposition to give serious consideration.

In an address on The Possible Improvement of the Human Breed under Existing Conditions of Law and Sentiment, Francis Galton grappled with this problem, but the proposals he put forward appear to be quite inadequate to meet the situation, and serve chiefly to emphasize the fact that before much can be accomplished "existing conditions of law and sentiment" will have to be changed. Galton's proposal of "granting diplomas to a select class of young men and women" on the basis of eugenic worth, and his advocacy of "the provision to exceptionally promising young couples of healthy and convenient houses at low rentals" would at best be likely to have only a slight effect upon the birth rate of the more promising members of the community. An experiment in accordance with the latter suggestion has been carried out by Mr. Alfred Dachert in the Jardins-Ungemach, near Strasbourg. Furnished houses at low rental are provided for properly qualified young couples with the aim of increasing their birth rate, and if after a reasonable interval the experiment proves unsuccessful, the sterile couples are required to give place to others. The most recent report on the working of the enterprise (Eugenics Review, October, 1935) indicates that it is having the desired results. The birth rate in the Jardins-Ungemach is I 28 per one thousand married women, as compared with a corresponding rate of 90 for the general population. To judge from their general health and physical development the children are distinctly above the average standard.

Philanthropists of the type of Mr. Dachert are rare. It cannot be expected that his example will be followed by many business executives unless conditions of sentiment 
among them on matters eugenic should undergo a very surprising change. As a rule, employers do not care a fig about the fertility of their employees. Certainly, an individual employer cannot be expected to hand out an additional stipend every time Mrs. Moriarty has another baby. The fact that the additional baby makes its appearance in a family already too large to be comfortably supported on the wages of the father concerns only this particular family, or possibly also the public charities. The Moriartys may be most excellent people whose increase in number would constitute a highly desirable contribution to the race. Assuming this to be true it would be to the advantage of the state that their numbers are not unduly curtailed, and that they enjoy a decent standard of living and receive a kind of education which helps them to become valuable members of the community. Granting that as a matter of social justice this family should secure really adequate support, the question arises as to who should pay for it. The individual employer cannot afford to pay the father more than his labor is worth. Since the state is benefited by the increase of this family, it has been proposed that the state or the community should defray at least a part of the cost of its support, instead of compelling poor Mr. Moriarty to bear the entire burden to the detriment of all of his offspring.

The family allowance system has been advocated as a means of removing in a measure the penalties of parenthood, and of securing a more just distribution of wages. To a certain extent it has been put into operation in several countries of Europe largely as a measure of relief to large families, and as a means of encouraging the increase of population in countries which sustained extensive losses during the World War. In France, where the system has been most fully carried out, family allowances are paid to wage earners in proportion to the number of children in a family, 


\section{HUMAN GENETICS AND ITS SOCIAL IMPORT}

both legitimate and illegitimate. The cost of the allowances is defrayed, not by the individual employer, but by a group of employers in the same industry or in the same region, so that the burden is widely distributed and does not tempt employers to discriminate against hiring men with large families. In 1932 a law was passed making it obligatory for all employers to contribute to some compensation fund. The number of such funds increased from 0 in 1920 to 230 in 1930 , when about $350,000,000$ francs were distributed to the families of wage earners. The stipends have scarcely been large enough to make any demonstrable increase in the birth rate, although they have often been a distinct relief to many struggling families. At first the labor unions looked upon the system with hostility or suspicion, but in general, they have now come to regard it with favor. The tendency of family allowances would be to reduce the basic wage for the single worker and to raise the income of workers with families. The total cost of labor need be no greater than under the usual system. Its chief effect is to secure a better distribution of income in relation to needs.

There would seem to be no inherent difficulty in extending the family allowance system until it came near meeting the additional cost incurred by the increased number of children in the family. If this is done it would doubtless have a considerable effect in increasing the birth rate. Baptist and Congregationalist missionaries are given a sufficient allowance for each child, so that a large family costs little more than a small one, a fact which may account for the considerably larger size of such families as compared with those of the regular ministers in these denominations. A few colleges, Roberts College in Istamboul, the American University at Beirut, and the London School of Economics afford family allowances for their faculty members. In the last institution they amount to thirty pounds annually from the sixth to the 
thirteenth year, and sixty pounds during the later period of schooling.

That it is possible to increase the birth supply by affording parents some relief from the financial difficulties incurred by having children is indicated by the success achieved through the legislation recently enacted in Germany. The new regime has made strenuous efforts to counteract the portentous decline of the birth rate in that country, not only through patriotic propaganda, but by a number of regulations for granting financial aid to parents. In I933 the German birth rate reached its lowest point of 14.7 per thousand inhabitants. The encouragements afforded to young couples by a system of loans to certain classes of workers, together with other measures, has apparently increased not only the number of marriages, but also the number of births. Eligible couples may receive a loan up to a thousand marks, to be repaid at the rate of $I$ per cent a month. The loans are made only when the wife has been employed at least nine months during the two preceding years, one object of the loan being the replacement of women workers by men. In 1933 there were $63 \mathrm{I}, \mathrm{I} 52$ marriages in Germany, or 9.7 per thousand inhabitants, whereas in I932 there were only 509,595 marriages, or 7.9 per thousand inhabitants. In 1934 the marriages increased to $73 \mathrm{I}, 43 \mathrm{I}$, or II.2 per thousand, and 37.4 per cent of the married couples received loans. The birth rate in 1934 rose suddenly from 14.7 to 18.0 per thousand, resulting in 47,825 more births than occurred in the first quarter of the previous year.

Burgdörfer states that "sixty per cent of the increase in births comes from marriages which were concluded with the help of loans. But these external measures are not the sole reason for the change." 'The decrease of unemployment and the changing attitude of the people are considered to be supplementary causes. A large part of the increase of births 


\section{HUMAN GENETICS AND ITS SOCIAL IMPORT}

occurred in urban families. There has been a marked decrease of abortions and illegitimate children. One reason for this is that the parents of an illegitimate child find in the loan system a means of taking care of the situation, and the temptation to resort to abortion is reduced. According to Mr. D. V. Glass (Eugenics Review, October, I935), there were between August, 1933, and March, I934, "part cancellations of loans in respect to the birth of 43 , IOI children who must have been conceived before marriage." A considerable proportion of these children, to judge from previous happenings, would have been prevented by abortion were the parents not able to receive a loan by marrying and to have a part of it remitted by having a child.

Whether the increased number of births resulting from the system of loans will improve the native qualities of the German people may well be doubted. The motive back of the enterprise is to secure greater military power through increase in numbers. In this Germany has succeeded, at least for the time being, in that her birth rate has shown a conspicuous rise, while that of most other European countries has continued to fall. From the eugenic standpoint the German experiment is of interest in showing that it is possible to raise the birth rate by relieving parents of a part of the financial burdens of childbearing. If the system promotes the fertility of certain classes of the working population, it should be possible to increase any desired class in much the same way.

It has been maintained that a family allowance system based merely upon the number of children without regard to the quality of their parentage would have a dysgenic influence, since the more undesirable elements of the community would be the most apt to respond to the financial inducements to fertility. On the other hand, it has been claimed that raising the standard of living among the poorer people 
of the community would result in lowering their birth rate. The lowest strata it is assumed do not artificially reduce their birth rate, and hence their fertility would not be increased by adding to their income. That indiscriminate family allowances would have a eugenic influence seems very doubtful. In order to insure the eugenic effect of such a system there would have to be some means by which family allowances were graduated according to the quality of the recipients. Several such schemes have been proposed. In his book Is America Safe for Democracy? Dr. William McDougall has recommended that members of certain "selected classes" be given to per cent of their income for every child under twenty years of age. "If such increase of income," says Dr. McDougall, "proportional to the earnings and to the number of children could be secured to each family of the selected classes, the eugenic effect would, I submit, be very great, far surpassing in this direction the effects of any other eugenic measure that has been proposed."

The system, according to Dr. McDougall, could readily be inaugurated with classes of state and municipal employees and then extended to schools and colleges and to such other occupational groups, membership in which is, on the whole, an indication of eugenic worth. The Eugenics Society of London has sponsored the family allowance system and has issued through its Council a statement in which it is declared that for both eugenic and social reasons "the economic motive for childlessness should by all possible means be diminished in all classes doing skilled work. The most potent means of effecting this end is a scientifically designed system of family allowances. The aim of such a system should be to equalize the standard of living between parents and nonparents doing equivalent work, within all grades affected, in such a way that the amounts recovered per child by each class of earner shall be proportional to the earnings." 


\section{HUMAN GENETICS AND ITS SOCIAL IMPORT}

Although the family allowance system is coming to be approved by an increasing number of publicists and economists, it is still very much of a controverted topic. It is opposed to the spirit of individualism, which is a very prevalent sentiment especially in the United States. The consideration given to certain favored classes would not be likely to meet the approval of the persons who were not benefited and who might be compelled indirectly to contribute to the support of those who were. The invidious distinctions involved in the system would, therefore, tend to make it unpopular in any democratically governed country, and would give a fine opportunity for demagogues to make an effective appeal to the interests of the less favored classes. A people with sufficient biological enlightenment might be willing to adopt a family allowance system that would have a real eugenic effect, but at present such a proposal would have a small chance of success. There would probably be less opposition to discriminating allowances paid by groups of employers than to a system managed by the government. If there is no social injustice in workers being paid more in some industries than in others, there should be none in the payment of unequal allowances for children. It is commonly assumed that the basic wage in any employment should be sufficient to support a man, his wife, and three children on a fair standard of comfort. If one recognizes the principle that wages should be determined, at least in part, by the needs of the worker's family, it would seem reasonable from the point of view of the worker and the state that wages should be adjusted to the size of the family, provided of course that the family is really a valuable one and not a social nuisance. The proponents of family allowances point out that, according to the theory of the basic wage mentioned above, many employees are being paid for families which they do not have. As a matter of fact, it is 
not feasible to pay all workers a wage sufficient to support a family of five on a fair subsistence level. Professor Paul Douglas estimates that if this were done in the United States, it would require 82 per cent of the total income of the country and thus leave an insufficient amount to cover other necessary expenditures. Other countries are in a similar economic condition, and the general result is that the children of a large proportion of wage earners' families suffer from inadequate support.

If incomes were so distributed that families with children received more than single workers, the general welfare of the population would doubtless be enhanced. One of the most important problems is the determination of the eugenic worth of those who receive the allowances. The attempt to gauge the genetic qualities of people, even provided that it could be done with the required degree of accuracy, would be difficult, not to say unpopular, in practice. If allowances were made on the basis of a certain percentage of the earned income, the general effect, it is alleged, would be to increase the birth rate of the persons who have achieved success and thus to rescue from extinction the abler classes of the population. In such a method there would be many cases in which eugenic breeding would not be promoted and in which encouragement would be given to undesirable stocks and withheld from many very desirable ones. But the general effect would probably be a considerable improvement over the present working of the differential birth rate. Several systems of family allowances have been devised some of which are eugenic in aim while others are planned solely to alleviate the financial burdens of large families regardless of quality. A family allowance scheme could readily be worked out that would have a strong influence for race betterment. The chief difficulty would be in persuading people to adopt it. 


\section{HUMAN GENETICS AND ITS SOCIAL IMPORT}

Just now people are not particularly interested in improving the hereditary qualities of the human breed. For the most part they know little about heredity, nor do they have much appreciation of the importance of hereditary differences among men. Until a few years ago the idea that the race might be improved by selective breeding scarcely entered the head of one person out of a million. To the great majority the idea is still foreign. And among the relatively few who have some notion of eugenics there prevails an extraordinary amount of misinformation and misunderstanding. The first step toward an effective eugenic reform is therefore education. And there is also need of further research devoted especially to settling questions of crucial importance in order that there may no longer be differences of opinion among qualified students of the subject. When knowledge of the fundamentals of eugenics is widely diffused, it may be expected to bear fruit in many ways. Negative eugenic measures, which are now: usually carried out in an ineffectual and half-hearted manner, will be much more thoroughly applied. People with hereditary defects will be more careful about passing their infirmities on to their descendants. Immigration will be more effectively regulated according to the eugenic worth of the entering aliens. There will be more discriminating choice in marriage and hence fewer persons with inferior endowments will be able to find mates. The influence that doctors, clergymen, and teachers exert in the interests of race betterment, which is now lamentably small as compared with what it might become, will be much more effective. There will be a greater sense of obligation on the part of those possessing superior hereditary qualities to see that their family strains are not suffered to die out. And people will be more favorably disposed toward measures that will encourage the increase of superior stocks. All these and other desirable changes might conceivably follow from the spread 
of eugenic education. But, of course, these changes may not occur. Instead, the trend of events may justify Bertrand Russell's prediction that for the next few hundred years "each generation will become stupider than its predecessors." Then, as we become more stupid we shall be less and less likely to remedy our racial ills. The situation offers a distinct challenge to our capacity for properly guiding the course of our biological development. We may fail in the task. But it is only through eugenic education that we can hope to succeed.

\section{Suggested Readings}

Darwin, L. ('25, '26). Douglas ('26). Fisher ('32). Galton ('o9). Hodson ('34). Holmes ('33), chaps. 6, 7 and Appendix F. Gosney and Popenoe ('29). McDougall ('2I), Appendix III, ('33). Popenoe and Johnson ('33), chaps. 7-9, 16-20. Wiggam ('24), chaps. I8, 19, 21, ('27), chap. 7.

\section{Questions}

I. List the hereditary defects which in your opinion should constitute a legal bar to marriage.

2. Do you think that any kinds of criminals (and if so what kinds) should be sterilized on either eugenic or other grounds?

3. In general what kinds of persons, if any, should be sterilized?

4. Is it justifiable to sterilize any classes of people on other than biological grounds?

5. If individuals outside of institutions should be sterilized how would you determine who should undergo the operation?

6. If a recessive trait is present in one-sixteenth of the population, what proportion of the population would be heterozygous for this trait? If all persons having the trait were prevented from reproduction, to what extent would it be reduced in the two following generations?

7. On the whole do you think that venereal diseases have a eugenic or a dysgenic effect? Give reasons.

8. List in one column the forces which are acting eugenically and in another those which are acting dysgenically.

9. Compare this list with a similar one made for primitive society.

ro. Should voluntary sterilization be regulated by law, or left to the individual and the medical profession? 


\section{HUMAN GENETICS AND ITS SOCIAL IMPORT}

II. What legal objections have been urged against sterilization?

12. Make a list of feasible measures for promoting race betterment.

13. Discuss the probable eugenic effects of indiscriminate family endowment.

14. List the arguments pro and con in regard to the eugenic influence of birth control. 


\section{Bibliography}

Obviously only a small part of the literature on the subjects treated in this volume can be included in a brief bibliography. A much more extensive collection consisting of over 10,000 titles may be found in A Bibliography of Eugenics by S. J. Holmes published by the University of California Press in 1924. After this date the literature on eugenics and related subjects has been published at intervals in the Bibliographia Eugenica appearing in Eugenical News. Many references on population problems are given in the works of Thompson ('35) and Lorimer and Osborn ('34), and also in the issues of Population Literature compiled by the Population Association of America. Literature lists on genetics are published in several recent books on the subject included in the following list, i.e., Babcock and Clausen, ('27), Castle ('30), Fasten ('35), Morgan ('19; '28), and Sinnott and Dunn ('32). Current literature on genetics is listed in Biological Abstracts.

AgAR, W. E. I93I. A Lamarckian experiment involving a hundred generations with negative results, Four. Exp. Biol., 8: 95-108.

Altenberg, E. I928. How we inherit, Holt, N.Y.

Armstrong, C. W. 1932. Practical family endowment, Eugenics Rev., 24: 107-II 4.

BABCock, E. B., and Clausen, R. E. I927. Genetics in relation to agriculture, McGraw-Hill, New York, 675 pp.

Baber, R. A., and Ross, E. A. 1924. Changes in the size of the American family in one generation, Univ. Wis. Studies, $99 \mathrm{pp}$.

BAKER, C. E. 1933. Rural-urban migration and the national welfare, Am. Assn. Geog., 23: 59-126.

BAKER, J. R. I926. Sex in man and animals, Routledge, London, I7I pp.

BARNEs, H. E. I 926. The repression of crime, Doran, N.Y. 382 pp. 


\section{HUMAN GENETICS AND ITS SOCIAL IMPORT}

Barnes, I. 1929. The inheritance of pigmentation in the American Negro, Human Biol., I: $32 \mathrm{I}-38 \mathrm{I}$.

Barr, M. W. 1904. Mental defectives, Blakiston, Philadelphia, 368 pp.

Baur, E., Fischer, E., and Lenz, F. I931. Human heredity, Macmillan New York.

BEck, P. G. 1934. Recent trends in the rural population of Ohio, Wooster Ohio, 4I pp.

Beeton, M., and Pearson, K. Igoo. Data for the problem of evolution in man. II. A first study of the inheritance of longevity and the selective death rate in man, Proc. Roy. Soc., 65:290-305, 1900. Also Four. Inst. Actuaries, London, 35: I I 2-I 29, I 899-1901.

- I90I. On the inheritance of the duration of life, and on the intensity of natural selection in man, Biometrika, I: 50-89.

BeLL, A. G. I9I8. The duration of life and the conditions associated with longevity, Washington, D.C., 59 pp.

Bernhardi, F. von. I9I4. Germany and the next war, Longmans, Green, New York, $288 \mathrm{pp}$.

Blacker, C. P. 1934. The chances of morbid inheritance, Lewis, London, $449 \mathrm{pp}$.

- 1934. Voluntary sterilization. Oxford Univ. Press, London, I 45 pp. Bolin, J. S., and Holmes, S. J. 1927. Marriage selection and scholarship, Four. Hered., 18:253-255.

Bonar, J. 1924. Malthus and his work, Macmillan, New York, $43^{8}$ pp.

- I931. Theories of population from Raleigh to Arthur Young, Greenburg, New York, 253 pp.

Bossard, J. H. S. I932. Residential propinquity in marriage selection, Am. Four. Soc., 38: 219-224.

Bowen, E. 1931. An hypothesis of population growth, Columbia Univ. Press, New York, 238 pp.

Brigham, C. C. 1923. A study of American intelligence, Princeton Univ. Press, $210 \mathrm{pp}$.

- 1930. Intelligence tests of immigrant groups, Psychol. Rev., 37: $156-165$.

Britten R. H. I934. Mortality rates by occupational classes in the United States, Publ. Health Reps., 49 (38), I IOI-I I I I.

Burgdörfer, F. I934. Sterben die weissen Völker? Callwey, Berlin, 90 pp. BURKs, B. S. 1928a. The relative influence of nature and nurture upon mental development, 27th Yearbook Nat. Soc. Study of Education, Part I, 219-316. 


\section{BIBLIOGRAPHY}

1928b. A summary of the literature on the determiners of the intelligent quotient and the educational quotient, op. cit., Part II, $248-353$.

Burt, C. 1925. The young delinquent, London Univ. Press, 643 pp.

Bushee, F. A. I933. Principles of sociology, Holt, New York, 577 pp.

Carpenter, N. I927. Immigrants and their children, Govt. Print. Off.,

Washington, D.C., 43 I pp.

Carr-Saunders, A. M. 1932. The population problem, Clarendon Press,

Oxford, 5 I6 pp.

-. 1926. Eugenics, Holt, New York.

- 1927. Differential fertility, Proc. World Pop. Conf., London, I $30-143$.

- I935. Eugenics in the light of population trends, Eugenics Rev., 27: I I -20 .

Castle, W. E. I926. The biological and social consequences of race crossing, Am. Four. Phys. Anthrop., 2: 145-156, 1926. Four Hered., I 5: 366-369, 1924. See op. cit. I7: 182-183, 1926.

-. I930. Genetics and eugenics, 4th ed., Harvard Univ. Press.

-. I930. Race mixture and physical disharmonies, Science, 7I: $603-606$.

Cattell, J. McK. I9I5-I917. Families of American men of science, Pop. Sci. Mon., 86: 504-515, I9I 5; Sci. Mon., 4: 248-262; 5: 368-377, 1917.

Chapman, J. C., and Wiggins, D. M. I925. Relation of family size to intelligence of offspring and socio-economic status of family, Ped. Seminary, 32: 414-421.

Charles, E. I 934. The twilight of parenthood, Watts, London, 266 pp.

- 1932. The practice of birth control. An analysis of the birth control experiences of 900 women, Williams and Norgate, London, I9o pp.

Clark, L. P., and Stowell, W. L. I9I3. A study of mortality in four thousand feebleminded and idiots, N.Y. Med. Four., 97:376-378.

Clarkson, R. D. 1927. Morison Lectures on mental deficiency, Edinburgh Med. Four., 34: 19-35, 6I-75, 200-215.

Conklin, E. G. I930. Heredity and environment in the development of men, 6th ed., Princeton Univ. Press.

- 1921. The direction of human evolution, Scribner, New York, $247 \mathrm{pp}$.

Conrad, H. S., and Jones, H. E. I932. A field study of the differential birth rate, Four. Am. Statist. Assn., 27: $153^{-1} 59$. 


\section{HUMAN GENETICS AND ITS SOCIAL IMPORT}

Constable, F. C. 1905. Poverty and hereditary genius, Fifield, London, I 49 pp.

Cooper, J. M. 1923. Birth control, Nat. Catholic Welfare Council, Washington, $95 \mathrm{pp}$.

Соок, O. F. I928. Quenching life on the farm, Four. Hered., I9: 440-452. Cotron, H. H. 1921. The defective, delinquent, and insane, Princeton Univ. Press, $201 \mathrm{pp}$.

Cowdry, E. V., and Embree, E. R. 1930. Human biology and racial welfare, Hoeber, New York. Vol. 2.

Cox, C. M. 1926. Genetic studies of genius. Early mental traits of 300 geniuses, Stanford Univ. Press.

Cox, H. 1922. The problem of population, Cape, London.

$\mathrm{C}_{\mathrm{REW}}, \mathrm{F}$. A. E. 1925. Animal genetics, Edinburgh, $420 \mathrm{pp}$.

- 1927a. Organic inheritance in man. London and Edinburgh.

- 1927b. The genetics of sexuality in animals. Cambridge Univ. Press.

- 1930. The inheritance of educability, Proc. 6th. Internat. Congress of Genetics, I: 121-1 34, 1932. See also Eugenics Rev., 22: 55-59.

Dachert, A. 1932. An experiment in eugenics, Eugenics Rev., 24: 207-214.

DAHLBERG, G. 1926. Twin births and twins from a hereditary point of view, Tidens, Stockholm, $296 \mathrm{pp}$.

- 1930. Inbreeding in man, Genetics, I4: 42 I-454.

Darwin, C. R. I 896. The origin of species, 6th ed., New York, 503 pp.

- I 868. Variation in animals and plants under domestication, 2 vols., New York.

- . 1871. The descent of man and selection in relation to sex, London.

Darwin, L. 1925. Family allowances, Eugenics Rev., 16: 276-278.

- 1926. The need for eugenic reform, Appleton, New York, 529 pp.

- 1929. What is eugenics? Am. Eugenics Soc., New York, 88 pp.

Daven port, C. B. Igi I. Heredity in relation to eugenics, Holt, New York, $298 \mathrm{pp}$.

- I9I3a. State laws limiting marriage selection, Bull. Eug. Rec. Off., 9: $66 \mathrm{pp}$.

- $1913 b$. Heredity of skin color in Negro-white crosses, Publ. Carnegie Inst., No. I88, Washington.

- 1927. Heredity of human eye color, Bibliographia Gen., 3:443-463.

- 1930. Sex linkage in man, Genetics, I 5: 40I-444.

Davenport, C. B., and Muncey, E. B. 1916. Huntington's chorea in relation to heredity and eugenics, Am. Four. Insanity, 73: 195-222, 1916. Also in Bull. Eug. Rec. Off., 17: 1916. 
Danenport, C. B., and Steggerda, M. 1929. Race crossing in Famaica, Publ. Carnegie Inst., No. 395, Washington, 5 I 6 pp.

Davis, J. J. 1925. Selective immigration, St. Paul, 227 pp.

Dayton, N. A. I93I-1932. Mortality in mental deficiency over a fourteen year period in Massachusetts. Analysis of 8,800 cases. Proc. $55^{\text {th }}$ Ann. Assn Study of Feeble-mindedness, 36: 127-212.

- 1935. Influence of size of family upon the characteristics of the mentally deficient, Am. Four. Psychiat., 91 : 799-832.

- 1930. Abnormal labor as an etiological factor in mental deficiency and other associated conditions, New England Four. Med., 203: 398-413.

Detlefson, J. A. The inheritance of acquired characters, Physiol. Rev., 5: 243-278, 1925 .

Doll, E. A., Phelps, W. M., and Melcher, R. T. 1932. Mental deficiency and head injuries, Macmillan, New York, $289 \mathrm{pp}$.

Douglas, P. H. 1925. Wages and the family, Chicago.

- I 262. "Family allowance systems and the future population." In Dublin, Population Problems, Chap. 18: 273-286, Boston.

Dublin, L. I. I9I7. Causes of death by occupation, Bull. 207: U.S. Bur. Labor Statist., $88 \mathrm{pp}$.

. 1925. The excesses of birth control, Metropolitan Life Ins. Co., N.Y., to pp.

Dublin, L. I. and LotkA, A. J. I925. On the true rate of natural increase, Four. Am. Statist. Assn., 20: 305-339.

- 1930. The true rate of natural increase of the population of the United States, Metron, 8: 107-I I9.

Dugdale, R. 1902. The Fukes, 7 th ed., Putnam, N.Y.

Duncan, H. G. I929. Race and population problems, Longmans, N.Y., $424 \mathrm{pp}$.

Dunlap, K. I920. Personal beauty and race betterment, Mosby, St. Louis, I 920, 95 pp. See also Psychol. Rev., May, I 918.

Dunn, L. C. 1932. Heredity and variation, Univ. Soc., N.Y.

Durham, F. M. and Woods, H. M. 1932. Alcohol and inheritance, H. M.

Stationery Off., London, 1932, 63 pp.

EAst, E. M. 1923. Mankind at the crossroads, Scribner's, N.Y., 360 pp.

- 1927. Heredity and human affairs, Scribner's, N.Y., 325 pp.

East, E. M., and Jones, D. F. I918. Inbreeding and outbreeding, Lippincott, Phila., 285 pp.

Edin, K. A. 1929. The birth rate changes, Eugenics Rev., 20: 258-266, 1929. See also Proc. World Pop. Conf., Geneva, 205-207, 1927. 


\section{HUMAN GENETICS AND ITS SOCIAL IMPORT}

Elderton, E. M. I909. The relative strength of nature and nurture, Eug Lab. Lect. Series, 3 .

Ellis, H. 1926. A study of British genius, Houghton Mifflin, Boston. Ellis, R. S. I928. The psychology of individual differences, New York. Estaвrook, A. H. I916. The Fukes in 1915, Publ. No. 240, Carnegie Inst. Washington, $85 \mathrm{pp}$.

- I923. "The Tribe of Ishmael." In Eugenics, genetics and the family, Williams and Wilkins, Baltimore, 398-404.

Estabrook, A. H., and Davenport, C. B. I9i2. The Nam family, Mem. Eug. Rec. Off., 2: 85 pp.

Evans, H. M., and Swezy, O. 1929. The chromosomes in man, sex and somatic, Mem. Univ. Calif., 9: $65 \mathrm{pp}$.

FaIrchild, H. P. 1930. Immigration, Macmillan, N.Y., 520 pp.

- I926. The melting pot mistake, Little, Brown, Boston, 260 pp.

- I927. Optimum population, Proc. World Pop. Conf., Geneva, $72-73$.

Fasten, N. 1935. Principles of genetics and eugenics, Ginn, Boston, 407 pp Field, J. A. I93I. Essays on population, Chicago Univ. Press.

Fisher, R. A. 1924. The elimination of mental defect, Eugenics Rev., I6: II 4 -II6.

- 1927. The elimination of mental defect, Four. Hered., I 8: 529-531.

- I930. The genetical theory of natural selection, London.

- 1932a. The social selection of human fertility, Oxford Univ. Press, $32 \mathrm{pp}$.

- I 9326 . Family allowances in the contemporary economic situation, Eugenics Rev., 24: 87-95.

Fraser, L. M. 1934. On the concept of an optimum in population theory, Population, I: No. 2, 37-42.

Freeman, F. N. 1928. The influence of the environment on the intelligence, school achievement, and conduct of foster children. 27th. Ann. Yearbook Nat. Soc. Study of Education, I: I03-21 8.

- I935. A study of twins as a method of investigating naturenurture, Four. Am. Statist. Assn., 30: 269-273.

Freeman, F. S. I934. Individual differences: the nature and causes of variations in intelligence and special abilities, Holt, N.Y. 355 pp.

Galton, F. I869. Hereditary genius, Macmillan, London. - I874. English men of science. Their nature and nurture, Macmillan, London.

-. 1883. Inquiries into human faculty, Macmillan, London.

-. I889. Natural inheritance, Macmillan, London. 
- 1909. Essays in eugenics, Eugenics Education Soc., London.

Galton, F., and Schuster, E. igo6. Noteworthy families, Murray, London.

Gamio, M. 1930. Mexican immigration into the United States, Chicago Univ. Press, $262 \mathrm{pp}$.

GARTH, T. R. I93I. Race psychology, a study of racial mental differences, McGraw-Hill, New York.

Gates, R. R. I929. Heredity in man, Macmillan, New York.

Gee, W. 193r. Qualitative selection in cityward migration, Am. Four. Soc., $37: 254-265$.

- 1933. A quantitative study of rural depopulation in a single township, Am. Four. Soc., 39: 210-221.

Gee, W. and Corson, J. J. I929. Rural depopulation in certain tidewater and piedmont areas of Virginia, Monogr. Univ. Va. Inst. for Research in Soc. Sci., 3 .

GinI, C. I926. Decline in the birth-rate and the "fecundability" of woman, Eugenics Rev., I7: 258-274.

Goddard, H. H. 1912. The Kallikak family, Macmillan, N.Y., I2I pp.

-. I9I 4. Feeble-mindedness, Macmillan, N.Y., 599 pp.

- 1920. Human efficiency and levels of intelligence, Princeton Univ. Press, I 28 pp.

Godwin, Wm. I793. An enquiry concerning political justice and its influence on general virtue and happiness, 2 vols., Robinson, London.

-. 1820. Of population, Longman, Hurst, Rees, Orme, and Brown, London, $626 \mathrm{pp}$.

Gordon, A. 1930. Inheritance of epilepsy, Med. Four. and Record, I31: 624-626.

Gosney, E. S., and Popenoe, P. 1929. Sterilization for human betterment, Macmillan, N.Y., 202 pp.

Gover, M. 1927. Mortality among the Negroes in the United States, Publ. Health Bull. No. 74, Washington.

- 1929. Increase of the Negro population in the United States. Human Biol., I: 263-273.

Grant, M. 1934. The conquest of a continent, Scribner's, New York.

Green, C. V. I929. Birth and death rates of the feeble-minded, Bull. Eug. Rec. Off., 26.

Gun, W. T. J. I928. Studies in hereditary ability, London.

GuYer, M. F. 1927. Being well-born, Bobbs-Merrill, Indianapolis.

Guyer, M. F. and Smith, E. A. 1920. Studies on cytolysins, II. Some prenatal effects of lens antibodies, Four. Exp. Zool., 31: 171-223. 


\section{HUMAN GENETICS AND ITS SOCIAL IMPORT}

- 1924. Further studies on inheritance of eye defects in rabbits, op. cit., 38: 449-475.

Hacker, E. 1929. Criminality and immigration, Four. Crim. Law and Criminology, 20: 429-443.

Haggerty, M. E. and NAsh, H. B. 1924. Mental capacity of children and parental occupation, Four. Ed. Psych., I5: 559-572.

Haldane, J. B. S. 1932. The inequality of man and other essays, Chatto and Windus, London. 295 pp.

Hankins, F. I931. The racial basis of.civilization, Knopf, New York, $375 \mathrm{pp}$.

Harper, R. M. 1928. Religion and family size, Four. Hered., I9: 169-173. Hart, H. 1925. Familial differential fecundity, Four. Am. Statist. Assn., 20: $25-30$.

- 1922. Differential fertility in Iowa, Univ. Iowa Studies in Child Welfare, 2: 39 pp.

Haynes, F. E. I930. Criminology, McGraw-Hill, N.Y.

Healy, W. 1928. Mental factors in crime, Ment. Hyg., 12: 761-767.

Heron, D. 1906. On the relation of fertility in man to social status, Drapers

Co. Res. Mems. Studies in National Deterioration, I, London, 22 pp.

Herrman, C. I930. Infant mortality and the survival of the fittest, Sci. Mon., 22: 25-29.

Herskovits, M. J. 1928. The American Negro, a study in race crossing, Knopf, N.Y., 92 pp.

- I930. Anthropometry of the American Negro, Columbia Univ. Contr. to Anthropology, New York, 2.

Himes, N. I932. Birth control in history and clinical perspective, Proc. Am. Acad. Polit. Soc. Sci., 160: 49-65.

Hirsch, N. D. M. I926. A study of natio-racial mental differences, Genet. Psych. Monogr. I, nos. 3 and 4.

- 1930. Twins: Heredity and environment, Harvard Univ. Press.

Hodson, C. B. S. 1934. Human sterilization to-day, Watts, London, 56 pp. Hogben, L. I931. Genetic principles in medicine and social science, Williams and Norgate, London, 230 pp.

- 1933. Nature and nurture, Williams and Norgate, London, I 4 pp. Hollingworth, L. S. 1926. Gifted children: Their nature and nurture, Macmillan, N.Y., 374 pp.

Holmes, S J. I921. The trend of the race: A study of present tendencies in the biological development of civilized mankind, Harcourt, Brace, N.Y., 396 pp. 
- I923. Studies in evolution and eugenics, Harcourt, Brace, N.Y., 26I pp.

- I 1924. The size of college families, four. Hered., I5: 407-415.

-. I926. Op. cit., I 7: 235-239.

- 1926. The sex ratio in infant mortality as an index of a selective death rate, Univ. Calif. Publ. Zool., 29: 267-303.

- I930a. Nature versus nurture in the development of the mind, Sci. Mon., 31: 245-252.

- I930b. Natural selection in man, Eugenics Rev., 22: 7-16.

- I930c. The biological trend of the Negro, Univ. Calif. Chronicle, 32: $3^{8-70 .}$

- I93I. Differential mortality in the American Negro, Human Biol., 3: 71-106, 203-244.

- I $1932 a$. Will birth control lead to extinction? Sci. Mon., 34: 247$25 \mathrm{I}$.

- 1932b. The changing effects of race competition, Science, 75: $201-208$.

-. I933. The eugenic predicament, Harcourt, Brace, N.Y., 232 pp.

Holmes, S. J., and PARKer, S. L. I930. The stabilized natural increase of the Negro, Four. Am. Statist. Assn., 26: I 59-17I.

Holzinger, K. J. I 935. The statistical evaluation of nature and nurture, Four. Am. Statist. Assn., 30: 249-256.

Hоoтon, E. A. 1932. Preliminary remarks on the anthropology of the American criminal, Proc. Am. Philos. Soc., 7 I : 349-355.

Hoover, G. I932. The quantitative optimum of population, Ann. Am. Acad. Polit. Soc. Sci., I62: I-8.

Humm, D. G. 1932. Mental diseases in siblings, Univ. Southern Calif. thesis, Los Angeles.

Hunt, H. R. 1930. Some biological aspects of war, Galton Publ. Co., N.Y., II $8 \mathrm{pp}$.

Huntington, E. I935. Tomorrow's children, Wiley N.Y., I39 pp.

Huntington, E., and Whitney, L. F. 1927. The Builders of America, Morrow, N.Y., $368 \mathrm{pp}$

Hurst, C. C. 1932. The mechanism of creative evolution, Cambridge Univ. Press.

Huxley, J. S., and Carr-Saunders, A. M. 1924. Absence of prenatal effects of lens-antibodies in rabbits, Brit. Four. Exp. Biol., I : $215^{-248}$. Ibsen, H. L., and Bushnell, L. D. I93I. Eye defects in rabbits, Four. Exp. Zool., 58: 401-422. 


\section{HUMAN GENETICS AND ITS SOCIAL IMPORT}

Jennings, H. S. 1930. The biological basis of human nature, Norton, N.Y. -1935. Genetics, Norton, N.Y.

Johnson, R. H. 1914. Marriage selection, four. Hered., 5: I02-I I0.

- 1923. "Mate selection." In Eugenics, genetics and the family, Williams and Wilkins, Baltimore, 416-425.

Jones, D. Caradog. 1932a. Mental deficiency in Merseysids, Eugenics Rev., 24: 97-105.

- 1932b. Differential class fertility, Eugenics Rev., 24: 175-190.

\section{London.}

Jones, C. Caradog, and Carr-Saunders, A. M. I927. Relation between intelligence and social status among orphan children, Brit. Four. Psych., 17: 343-364.

Jones, H. E. I929. Homogamy in mental test abilities, Am. Four. Soc., $35: 369-382$.

Jones, H. E., Conrad H. S., and Blanchard, M. B. I932. Environmental handicap in mental test performance, Univ. Calif. Publ. in Psychol., 5: 63-99.

Jordan, D. S. I907. The human harvest, Beacon Press, N.Y.

- 1915. War and the breed, Beacon Press, N.Y.

Jordan, D. S. and Jordan, H. E. 1914. War's aftermath, Houghton Mifflin, Boston.

Kammerer, P. I924. The inheritance of acquired characters, Boni and Liveright, N.Y., 4I 4 pp.

Key, W. E. 1920. Heredity and social fitness, Carnegie Inst. Publ. 296, $102 \mathrm{pp}$.

KirKPATrick, C. I926. Intelligence and immigration, Williams and Wilkins, Baltimore.

Kiser, C. V. I933. Trends in the fertility of social classes from 1900 to I910, Human Biol., 5; 256-273.

Knibis, G. H. 1928. The shadow of the world's future, Benn, London, I 31 pp. Kuczynski, R. R. 1928. The balance of births and deaths. I. Western and Northern Europe, Macmillan, N.Y.

- 1930. The balance of births and deaths. II. Eastern and Southern Europe, Brookings Inst., Washington, 1930.

- 1932. Fertility and reproduction, Falcon Press, N.Y., 94 pp.

Landman, J. H. 1932. Human sterilization, Macmillan, N.Y. $43^{1}$ pp.

Lange, J. 1930. Crime as destiny, Boni, N.Y.

Laughlin, H. H. 1926. Eugenical sterilization, Am. Eugenics Soc., New Haven. 
Lauterbach, C. E. 1925. Studies in twin resemblance, Genetics, IO: 525568.

LaWrence, E. M. 1931. An investigation into the relation between intelligence and inheritance, Brit. Jour. Psych. Monogr. Suppl. no. I6, London.

LEAHY, A. M. 1935. A study of adopted children as a method of investigating nature-nurture, Four. Am. Statist. Assn., 30: 28 I-287.

LEGRAS, A. M. Psychose en criminaliteit bij tweelingen, Utrecht, 1932.

Lennes, N. J. I927. Whither democracy? Harpers, N.Y., 370 pp.

Lentz, T. I927. Relation of I.Q. to size of family, Four. Ed. Psych., I8: 486-496.

Lenz, F. 1927. Ueber die biologischen Grundlagen der Erziehung, Lehmann, Munich.

Lidbetter, E. J. I934. Heredity and the social problem group, Vol. I, Arnold, London.

Lindsey, A. W. I932. A textbook of genetics, Macmillan, N.Y., 354 pp.

Lombroso, C. I91 I. Crime. Its causes and remedies, Little, Brown, Boston, $47 \mathrm{I} \mathrm{pp.}$

Lorimer, F. I933. Factors influencing ratios of children to native-white women in the United States, I900 and 1930, 2uart. Bull. Milbank Mem. Fund, i I no. 3, suppl.

Lorimer, F. and Osborn, F. I934. Dynamics of population, N.Y., 46I pp. Lotk A, A. J. I 928. Studies in American marriage, Proc. Nat. Acad. Sci., I4: 99-108.

LudovicI, A. M. 1933. Eugenics and consanguineous marriages, Eugenics Rev., 25: 147-155.

-. I934. The choice of a mate, Lane, London, I934, 5 Io pp.

Lundborg, H. 193I. Die Rassenmischung beim Menschen, Bibliog. Genetica, 8: 1-226. (Full bibliography.)

McDougall, W. I921. Is America safe for democracy? Scribner's, N.Y., $218 \mathrm{pp}$.

- I927. An experiment for testing the hypothesis of Lamarck, Brit. Four. Psyckol. 17: 267-304.

- 1930. Second report on a Lamarckian experiment, l.c. 20: 201-218.

- 1933. Family allowances as a eugenic measure. Character and Personality, 2: 99-II6.

MacPherson, J. I929. The futility of sterilizing mental defectives,

Nineteenth Century, 106: 805-8 2.

Mallet, B. I931. The social problem group, Eugenics Rev., 23: 203-206.

Malthus, T. R. - An essay on population, 2 vols., Dutton, N.Y. 


\section{HUMAN GENETICS AND ITS SOCIAL IMPORT}

Malzberg, B. I 932. Life tables for patients with mental diseases, Four. Am. Statist. Assn. Suppl., 27: 160-174. 1932. Psychiat. 2uart., 6: 226-24I. Merriman, W. E. 1933. Psychoses in identical twins, Psychiat. 2uart., 7: $37-49$.

Miller, H. C. 1931. Further study of the Hill Folk, Eugenical News, I6: IO9-IIO.

M Joen, J. A. 1926. Biological consequences of race-crossing, Four. Hered., I7: 175-185.

Moore, E. I933. A bibliography of differential fertility, Edinburgh, 97 pp.

-. 1934. Heredity, mainly human, Chapman and Hall, London, $343 \mathrm{pp}$.

Morgan, T. H. I914. Heredity and sex, Columbia Univ. Press, N.Y., $282 \mathrm{pp}$.

- I9I9. The physical basis of heredity, Lippincott, Phila., 305 pp.

- I 1928. The theory of the gene, Yale Univ. Press, New Haven, 343 pp. Muller, H. J. 1925. Mental traits and heredity, Four. Hered., 16: 433-448. - 1929. The method of evolution, Sci. Mon., 29:48 I-505.

Murchison, C. 1926. Criminal intelligence, Clark Univ. Press, Worcester.

Myreson, A. I925. The inheritance of mental diseases, Williams and Wilkins, Baltimore.

Newman, H. H. I93I. Differences between conjoined twins, Four. Hered., 22: $201-215$.

-1932. Evolution, genetics and eugenics, ed. 3, Univ. Chicago Press.

- I 932. Mental and physical traits of identical twins reared apart, Four. Hered., 20: nos. 2, 3 and 4, 1929; 23: 3-18.

- I I933. The effects of hereditary and environmental differences upon human personality as revealed by studies of twins, Am. Nat., 67: 193-205.

Newsholme, A. 1923. The elements of vital statistics, rev. ed., Allen and Unwin, London. 623 pp.

Nicolai, G. F. I9I 8. The biology of war, Century Co., New York, 553 pp.

Notestein, F. W. I93I. Differential age at marriage according to social class, Am. Four. Soc., 37:22-48.

- I931. The decrease in size of families from 1890 to 1910, 2uart. Bull. Milbank Mem. Fund, 9: 181-188.

- 1933. The differential rate of increase among the social classes of the American population, Social Forces, I2: 17-33.

Notestein, F. W., and Sallume, X. 1932. The fertility of specific occupational groups in an urban population, 2uart. Bull. Milbank Mem. Fund, 10: 120-130. 
Ogburn, W. F., and Tibitts, C. I932. Birth rates and social classes, Social Forces, 8: 1-IO.

Osвorn, D. I9I6. Inheritance of baldness, Four. Hered., 7: 347-355.

Osborn, F. 1933. Characteristics and differential fertility of American population groups, Social Forces, I 2: 8-16.

Pearl, R. 1924. Studies in human biology, Williams and Wilkins, Baltimore, $653 \mathrm{pp}$.

- - 1925. The biology of population growth, Knopf, N.Y., 260 pp.

- 1927. Differential fertility, 2uart. Rev. Biol., 2: 102-1 18.

- 1930. Introduction to medical biometry and statistics, Saunders, Philadelphia.

- 1932. Contraception and fertility in 2,000 women, Human Biol., 4: 363-407.

- I934. Contraception and fertility in 4,945 married women, l.c. 6 : 355-401.

Pearl, R. and Pearl, R. DeW. 1934. The ancestry of the long-lived, Johns Hopkins Press, Baltimore, I68 pp.

Pearson, K. 1905. National life from the standpoint of science, 2d. ed., Eugenics Lab. Lect. Series, II, I905, 106 pp.

- IgIo. Nature and nurture, the problem of the future, Eugenics Lab. Lect. Series 5, 3I pp.

- I9I2. The academic aspect of the science of national eugenics, Eugenics Lab. Lect. Series, 7, 27 pp.

- I912. Darwinism, medical progress and eugenics, Eugenics Lab. Lect. Series, 9, 29 pp.

- 1927. The right of the unborn child, Eugenics Lab. Lect. Series, I4, $26 \mathrm{pp}$.

Pease, M. S. I934. Professor McDougall's Lamarckian experiment, Eugenics Rev., 25, 253-254.

Penrose, E. F. I934. Population theories and their application with special reference to Fapan, Stanford Univ., 347 pp.

Penrose, L. S. I 933. Mental defect, Sedgwick and Jackson, London.

- 1933. A study of the inheritance of intelligence. The analysis of 100 families containing subcultural mental defectives, Brit. Four. Psych., 24: I-I 9 .

Perkins, H. F. I929. The children of feeble-minded and insane parents, 3d Ann. Rep., Eugenics Survey of Vermont, 1929.

Pitt-Rivers, G. M. 1927. The clash of culture and the contact of races, Routledge, London, $3{ }^{12}$ pp. 
Ploetz, A. 1909. Lebensdauer der Eltern und Kindersterblichkeit, Arch. Rass.-und Ges.-Biol., 6: 33-43.

Popenoe, P. I929. The child's heredity, Williams and Wilkins, Baltimore $316 \mathrm{pp}$.

- 1930. The fecundity of the feeble-minded, Arch. Rass.-und Ges.Biol., 24: 29I-298.

Popenoe, P., and Johnson, R. S. 1933. Applied eugenics, 2d ed., Macmillan, New York.

Porteus, S. D. I930. Race and social differences in performance tests, Genet. Psych. Monogr. Worcester, 8, no. 2, 93-208.

Porteus, S. D., and $\mathrm{B}_{\mathrm{ABcock}}$, M. E. 1926. Temperament and race, Badger, Boston, $364 \mathrm{pp}$.

Rathbone, E. 1927. The disinherited family, 3d. ed., Allen and Unwin, London.

- 1927. The ethics and economics of family endowment, Epworth Press, London, i 8 pp.

Reuter, E. B. I918. The mulatto in the United States, Badger, Boston.

-. 1923. Population problems, Lippincott., Phila., $33^{8} \mathrm{pp}$.

- I927. The American race problem, Crowell, N.Y., 448 pp.

- I93I. Race mixture, McGraw-Hill, N.Y., 23I pp.

Rice, T. B. I 929. Race hygiene, Macmillan, N.Y., 376 pp.

Robisns, L. I927. "The optimum theory of population." In London Essays on Economics, etc., 124-134, Routledge, London.

Roberts, E. H. I927. Population problems of the Pacific, Routledge, London, 4II pp.

Rollins, W. A. I929. Fertility of college graduates, four. Hered., 20: 425-427.

- 1930. The effect of immigration on the birth rate of the natives, Four. Hered., 21: 387-402.

Root, W. T. No date. Psychological and educational survey of 1,916 prisoners in the Western Penitentiary of Pennsylvania, Western Penitentiary, Pa., 246 pp.

Roannoff, A. J., Handy, L. M., and Plesset, I. R. 1935. The etiology of manic-depressive syndromes with special reference to their occurrence in twins, Am. Four. Psychiat., 91: 725-762. schizophrenic psychoses with special reference to their occurrence in twins Am Four. Psychia:, 91: 247-286.

- - $\longrightarrow$, and Rosanoff, I. A. 1934. Criminality and delinquency in twins, four. Crim. Law and Criminol., 24: 923-934. 
Ross, E. A. I927. Standing room only, Century Co., N.Y., 368 pp.

Ryan, J. A. I927. Human sterilization, Washington, Nat. Catholic Welfare Conf.

SAller, K. I932. Erblichkeitslehre und Eugenik, Springer, Berlin, 307 pp.

Sallume, X. and Notestein, F. W. I932. Trends in size of families completed prior to I9IO in various social classes, Am. Four. Soc., 38 : $398-408$.

Sanger, M. 1927. Proc. World Pop. Conf. London, Arnold, 383 pp.

- I928. Motherhood in bondage, Brentano, N.Y., 445 pp.

Schiller, B. I932. A quantitative analysis of marriage selection in a small group, Four. Soc. Psych., 3: 297-318.

Schiller, F. C. S. 1932. Social decay and eugenical reform, Constable, London, I64 pp.

Schwesinger, G. C. I933. Heredity and environment, Macmillan, N. Y.

Sharp, L. I 934. Introduction to cytology, 3d ed., McGraw-Hill, New York.

Shull, A. F. I93. Heredity, McGraw-Hill, New York.

Sinnotr, E. W., and Dunn, L. C. I932. Principles of genetics, McGraw-Hill, New York.

Sмith, M. R. I900. Status of college and non college women, Publ. Am.

Statist. Assn., 7: I-26.

SNow, E. C. IgII. On the intensity of natural selection in man, Drapers' Co.

Memoirs, Studies in national deterioration, London, 7:43 pp.

SNYDER, L. W. I927. Blood grouping in relation to clinical and legal medicine,

Williams and Wilkins, Baltimore.

Sorokin, P. I927. Social mobility, Harper, N.Y.

Sorokin, P., and Zimmerman, C. C. I929. Principles of rural-urban sociology, Holt, N.Y., xiv $+65^{2} \mathrm{pp}$.

Spearman, C. E. I927. The abilities of man; their nature and measurement, Macmillan, N.Y.

Stockard, C. R. I93I. The physical basis of personality, Norton, N.Y.

StOdDaRd, L. I920. The rising tide of color, Scribner's, N.Y., 320 pp.

Sydenstricker, E. I 933. "The vitality of the American people." In Recent social trends in the United States, I, Chap. 12: 602-660, McGraw-Hill, N.Y.

Sydenstricker, E., and Notestein, F. W. i930. Differential fertility according to social class, Four. Am. Statist. Assn., 25:9-32.

Taylor, P. I930. Mexican labor in the United States, Univ. Calif. Publ. in Economics, 6: nos. I-5, I928-I929, 7, no. I.

Terman, L. M. I925. Genetic studies of genius. Vol. I, Mental and physical traits of a thousand gifted children, Stanford Univ. Press. 


\section{HUMAN GENETICS AND ITS SOCIAL IMPORT}

Thompson, W. S. I917. Race suicide in the United States, Sci. Mon., 5; $22-35,154-165,258-269$.

- I 925 . Size of families'from which college students come, four. Am. Statist. Assn., 20: 48 I-495.

- I929a. Danger spots in world population, Knopf, N.Y., 343 pp.

- I $929 b$. Natural selection in the process of population growth, Human Biol., I: 503-513.

- I93I. Ratio of women to children in the United States, 1920, Census Monogr., I I : Washington.

\section{- I935. Population problems, 2d ed., McGraw-Hill, N.Y., 500 pp.}

Thompson, W. S., and Whelpton, P. K. 1933. Population trends in the United States, McGraw-Hill, N.Y. 4I 5 pp.

Tredgold, F. A. I930. Mental deficiency, Wood, N.Y.

Ungern-Sternberg, R. von. I93I. The causes of the decline in birth-rate within the European sphere of civilization, Eugenics Research Assn. Monogr., 4: 202 pp.

Verschuer, O. von. 1932. Ergebnisse der Zwillingsforschung, Verhand. $d$.

Ges.f. phys. Anthrop., 6: I-65.

Vibart, H. H. R. I926. Family allowance in practice, King, London.

Wagner-Manslau, W. I93I. Heredity as an explanation of the declining birth rate, Eugenics Research Assn. Monogr., 6: 48 pp.

-. 1934. Eugenics Rev., 26: 193-198.

- I935. The inheritance of fertility, Ann. Eugenics, 6: 225-25 I.

Walter, H. E. I930. Genetics, Macmillan, N.Y.

Weismann, A. I891, 1892. Essays on Heredity, Clarendon Press, Oxford,

Vol. I. 1891, 47I pp.; Vol. 2. I892, 226 pp.

- I 1893. The germ plasm, Scott, London, $477 \mathrm{pp.}$

Wells, H. G., Huxley, J. S., and Wells, G. P. I931. The science of life, Doubleday, Doran, N.Y.

Whelpton, P. K. I929. Differentials in true natural increase, four. Am. Statist. Assn., 24: 233-249.

- I930, I933. Population; trends in differentials of true increase and age composition, Am. Four. Soc., 35:870-880, I930; 37:855-86 I, 1932. Whetten, N. L. I933. Education and size of family, Four. Hered., 24: $275^{-278 .}$

Whipple, G. C. 1923. Vital statistics, 2d ed., Wiley, N.Y.

White, F. W. 1928. Natural and social selection, Eugenics Rev., 20: 98-104.

Whitney, L. F. 1934. The case for sterilization, Stokes, N.Y., 309 pp. 
Wiggam, A. E. 1924. The fruit of the family tree, Bobbs-Merrill, Indianapolis.

- 1927. The next age of man, Bobbs-Merrill, Indianapolis.

- 1928. Exploring your mind, Bobbs-Merrill, Indianapolis.

Willcox, W. F. I 929-I 931. International migrations, 2 vols., Nat. Bureau Econ. Research, N.Y.

- 1933. Introduction to the vital statistics of the United States, I 900 to I930, Bureau of the Census, Washington, I $37 \mathrm{pp}$.

Willoughby, R. R. I933. Somatic homogamy in man, Human Biol., 5: 690-705.

Wilson, E. B. I928. The cell in development and heredity, $3 \mathrm{~d}$ ed., Macmillan, N.Y.

Wingfield, A. H. I928. Twins and orphans, Dent, London and Toronto, I $27 \mathrm{pp}$.

Winship, A. E. 1900. Fukes-Edwards, Myers, Harrisburg, 88 pp.

Wolfe, A. B. I928-1929. The population problem since the World War, Four. Polit. Econ., 36-37: 529-559, 662-685, 87-1 20, I 928, I 929.

- 1934. The criterion of optimum population, Am. Four. Soc., 39: 585-599.

Woods, F. A. 1928. Successful men have larger families, four. Hered., I9: $27 \mathrm{I}-280$.

Wright, H. I923. Population, Harcourt, Brace, N.Y., I80 pp.

Yerkes, R. M. I921. Psychological examining in the United States Army, Mem. Nat. Acad. Sci., 15: Washington.

Young, T. E. I905. On centenarians, London. 



\section{Index}

A

Abadie, J., inheritance of epilepsy, I 46

Abnormal abdomen in Drosophila, 62

Abortion, 268, 36r

Acquired characters, inheritance of, $19 f$.

Achondroplastic dwarfs, Ioo

Adkinson, J., Io6

Adler, H. M., 174, I75

Agar, W. E., 387

Agriculture, 320, 32I

Albinism, inheritance of, 98-100

Alcohol, inherited effects of, $80-82,85$

Alcoholism, I37, 1 56, 184-186, 263

Alexander, 204

Altenberg, E., 39, 387

Amaurotic idiocy, I29, I 5 I , 257, 265

Amentia, 127

Amitosis, 6, 17

Andalusian fowl, 59, 60, 64

Anthrax, immunity to, 105, 106

Aristotle, views on eugenics, 360, 36I

Armstrong, C. W., 387

Army mental tests, I 24, I6I , 342, 343

Ascaris, 8, 9

Ashby, H. T., on infant mortality, 27I

Asia, emigration from, 332, 333

Assortative mating, 193-195, 373, 374

Asthma, inherited diathesis to, 63,64 , Ió6, 108

Atavism, 172

Australia, birth rate, 199

Average deviation, $9 \mathrm{I}$

Ayres, 155

\section{B}

Babcock, E. B., 39, 88, 96, 387

Babcock, M. E., 400
Baber, R. A., 220, 387

Bacon, F., 290

Bagg, H., 84

Baker, C. E., 387

Baker, J. R., $3^{87}$

Baldness, heredity, 99

Barnes, H. E., on prison system, I I I, $3^{87}$

Barnes, I., 388

Barr, M. W., 259, 382

Barries, K., 224

Bateson, W., 59, 66

Batrachoseps, synapsis, 69

Baur, E., I Iо, I 47, 388

Beck, P. G., 388

Beeton, M., 255, 388

Bell, A. G., inherited deafness, I04, 256,388

Belling, J., I 2

Bere, M., 345

Berlin, birth rate, 208, 21 3, 229, 324

Bernhardi, F. von, 282, 283, 288, 388

Bertillon, J., 208

Besant, A., 206, 207, $3^{6} 5$

Beta test, 342,343

Bidder's organ, 49

Binet, A., 173

mental tests, $167,175,345$

Biometry, $87 f f$.

Birth control, 206, 365, 366

Birth injuries, I 26, I 27, I 43, I 56, 268

Birth rate, 196 ff , 246, 249, 287, 297$300,3^{14}-3^{17}, 3^{21-325}, 337-340$, $365,366,375,37^{8-381}$

Blacker, C. P., I Iо, 388

Blanchard, M. B., 327, 396

Blanton, S., I 55

Blending inheritance, 54,55

Blindness, inheritance, I09, I46, 204 


\section{HUMAN GENETICS AND I'TS SOCIAL IMPOR'T}

Bluhm, A., hereditary effect of alcohol, $8 \mathrm{I}$

Boas, F., 353

Bolin, J. S., 388

Bonar, J., 388

Bossard, J. H. S., $3^{88}$

Bowen, E., 388

Bowman, J. T., 146

Box bill, 339

Bradford, intelligence and family size, 225

Bradlaugh, C., 206, 207, 365

Bridges, C. B., 69, 73

Brigham, C. C., 348,388

Britten, R. H., 388

Bronner, A. F., 178

Brooks, W. K., 90, $9^{1}$

Buck vs. Bell, test of sterilization statute, $37 \mathrm{I}$

Buckle, T. H., I 2

Bulgaria, age for marriage in, 201

Burbank, L., 86

Burgdörfer, F., 21 7, 301, 302, 31 5, 324, 379,388

Burks, B. S., I60, I61, I69, 388

Burt, C., I 77, 389

Bushee, F. A., 389

Bushnell, L. D., 83, 395

Butt, N. I., 230

Buttercup, variability, 89

\section{C}

California, sterilization in, 372, 373

California, University of, family size of students, 209, 219

marriage of graduates, 218

Campanella, T., 362

Cancer, inheritance, 106-108

Carlile, R., 207, 365

Carpenter, N., 389

Carr-Saunders, A. M., 83, 163, 164, 310, $389,395,396$

Castle, W. E., 352, 357, 387, $3^{89}$

Cataract, inheritance, I02, 103

Catholics, birth rate, $2 \mathrm{II}-2 \mathrm{I} 3$

Cattell, J. McK., I9I, 260, 389
Cell theory, 2-4

Cells, 3-8, I I, I 4, I 5

Centenarians, 242

Centrosome, $5^{-7}$

Chapman, J. C., 225, 389

Charles, E., 236, $3^{89}$

Chiasmas, 71

China, $33^{2}$

Chromatin, 6, 9

Chromomeres, II, I 2, I6

Chromosomes, 6-18, 35-40, 42-47, $66-75,77,78,90$, I I 5

Cities, growth of, 3 I9 ff. mortality in, 248-25 I

Clark, L. P., 259, 389

Clarke, C. K., and Mc Arthur, J. W., Huntington's chorea, I 4 I

Clarkson, R. D., $3^{89}$

Clausen, R. E., 39, 88, 96, 387

Cockayne, E. A., IO4

Coefficient of variability, 92, 93

Coefficients of correlation (see Correlation)

College graduates, birth rates, $217^{-220}$, 231

marriage rates, 191, 217, 218, 231

Color blindness, I02-104, 108

Colvin Hollow, 326

Complementary factors, $55^{-58}$

Condorcet, A. N., 290

Conklin, E. G., 39, 56, 389

Conrad, H. S., 230, 327, 389, 396

Conrad, K., I 45

Consanguinity, $349 f f$.

Constable, F. C., 390

Cook, O. F., 390

Cooper, J. M., 390

Correlation, 94-97, 106, 116, 117,153 , 154,256

Correns, C., 29, 59

Corson, J. J., 393

Cotton, H. H., I 37,390

Cousins, marriage of, $35^{\circ}$

Cowdry, E. V., 389

Cox, C. M., I 57, I 58, $3^{89}$

Cox, H., 288, 389

Crepidula, sex determination, 47,48 
Cretins, II 4 , II 5

Crew, F. A. E., 26, 48, I10, 390

Crime, I 7 I ff., I $83^{-1} 86$

Cross breeding, $349 f f$.

Crossing over, 68-7I

Crum, F. S., 266

Cumulative factors, $5^{8}$

Cytology, 2 ff.

Cytoplasm, $4^{-8}$

\section{D}

Dachert, A., 376, 390

Dahlberg, G., I 21, I 24, 390

Danielson, F. H., 326

Darwin, C. R., 20, 21, 23, 28, 57, 72, I $53,188,189,195,253,255,272$, 291, 293, 350, 362, 390

Darwin, L., I 53, 195, 385, 390

Darwinism, 276, 277

Datura, 72

Davenport, C. B., 55, 110, 139, 187, $326,35^{2}, 357,364,390,39^{1}$

Davis, J. J., 39I

Davis, R. A., I6I

Dawson, S., 225

Dayton, N. A., I 27, 235, 259, 39I

Deafness, I03, 104, 108, 156

Death rates, 199-201, $23^{8} \not f$., 298-300, $3^{1} 4,3^{1} 5,3^{21-}-324$

Delinquency, $177^{-1} 79$

Dementia praecox, I $3^{8-1} 4$ O, I 42 , I 43

Detlefson, J. A., 27, 391

De Vries, H., 27, 89

Diabetes, inheritance of, 63, 105, 257

Diehl, K., 124

Doll, E. A., 147, 391

Dominance, 30, 3I, 58, 59, 6I-64, 99IOI

Domm, L. V., $4^{8}$

Donkin, H. B., I 73

Dorn, H. F., 250

Douglas P., 383, 385, 391

Drosophila, 8, I2, I3 $25,45,46,61$, $62,66-75,78,85,109$

Dublin, L. I., 21 7, 272, 303, 31 8, 39 r

Dumont, A., 210
Duncan, H. G., 318, 39I

Dunlap, K., I89, I95, 39 I

Dunn, L. C., 39, 49, 64, 387, 391, 40I

Durham, F. W., 82, 39I

Dwarfs, I00, II 5

E

East, E. M., 21 4, 295, 318, 348, 357, 39I

Edin, K., 391

Elderton, E. M., I 16, 392

Ellis, H., I70, I89, 392

Ellis, R. S., 392

Embree, E. R., 389

Emigration, 305, 330-332

England and Wales, age at marriage, 201 birth rate in, 199, 207, 208 occupational mortality in, 261

Epilepsy, in criminals, 178 inheritance, IIO, $130,143^{-1} 4^{6}$

Estabrook, A. H., I 83, I 87, 392

Eugenics, I49, 359ff.

Eugenics Record Office, $36_{4}$

Eugenics Society, 363, 381

Europe, birth rate, 200

emigration from, 305, 331-337

marriages in, 202

population growth in, $297,298,300-$ $3 \circ 3$

Evans, H. M., 9, $39^{2}$

Eye color, 52, 98, 99, 113,120

Eye defects, 52, 82, 83, 102, 103

F

Fairchild, H. P., 308, 348, $39^{2}$

Family allowances, $377 . f$.

Farr, Wm., 248

Farr's law, 248

Fasten, N., 387, $39^{2}$

Fecundity, definition, 197

Feeble-minded, birth rate, $23^{1-236}$ death rate, 259, 260, 269

Feeble-mindedness, 64, I $26 f f$., 346, 370-373

Fernald, W. S., 173

Fertility, $196 f f ., 323,324,326,381,388$ 


\section{HUMAN GENETICS AND ITS SOCIAL IMPOR'T}

Fertilization, I4, I 5, I7

Fischer, E., I 10, I 47, 388

Fisher, R. A., 214, 385, $39^{2}$

Focal infections, role in insanity, 137

Foster children, $160,161,163,164$

Four o'clocks, inheritance of color, 59

Fragility of bone, inheritance, 108

France, birth rate, $198,205,212-214$

population growth, 300-302

Franklin, B., 290

Fraser, L. M., 392

Freeman, F. N., 160, 170, 392

Freeman, F. S., 170, $39^{2}$

Frequency curves, 88-90

Fruit fly, 12, I3, 39, 66-75, 80, 85

(See also Drosophila)

Fundulus, I I, I3, I 8

\section{G}

Galton, F., 87, 94, 98, I13, I17, I52, 170, 193, 198, 260, 325, 362-364, $376,385,392,393$

Galton Laboratory, $87,363,364$

Gametogenesis, I 5

Gamio, M., 344, 393

Garth, T. R., 354, 393

Gates, R. R., I10, I 47, 393

Gee, W., 326, 328, 393

Genes, 38, 39, 52, 54, 55, 57, 58, 60-63, 79, I00, IO8-I10, I I 3-I I 5, I 33 , I 34 , 357

Gene mutations, 78, 79

Genetics, I, 2

Genius, inheritance, $15^{2} f f$.

Germ plasm, continuity, 22, 23

Germany, age at marriage in, $20 \mathrm{I}$

birth rate for, 199, 21 3, 216, 379

sterilization in, 373

Gesell, A., I67, I68

Gini, C., 203, 393

Glass, D. V., 380

Glaucoma, heredity, 102, 103

Glioma of retina, 107

Goddard, H. H., I32, I33, I47, I84, I87, 393

Godwin, W., 290, 291, 393
Gonorrhea, 204, 205, 268

Gordon, A., I 43, I44, 393

Goring, C., 117,172

Gosney, E. S., 385, 393

Gould, H. N., 47

Gover, M., 393

Grant, M., 348, 393

Grassl, J., 217

Graunt, J., 239

Greeks, ideas on eugenics, 359-36I

Green, C. V., 232, 393

Grew, N., 2

Gun, W. T. J., 393

Guyer, M. F., 27, 82, 83, 85, I8r, 393

H

Hacker, E., 394

Haeckel, E., 3

Haemophilia, 5 I, 257

Haggerty, M. E., 394

Hair color, 99, I 20

Haldane, J. B. S., 180, 394

Hall of Fame, I 59

Hamburg, birth rate, 229, 325 venereal diseases in, 205

Handy, L. M., I42, I80, 400

Hankins, F. H., 394

Hansen, G., 325

Hanson, F. B., 8I

Hare lip, 108

Harper, R. M., 335, 394

Hart, H., 394

Harvard, birth rate of graduates, 217 , 231

losses in war, 280

Haupt, A. W., 32

Hawaii, population of, $3^{12}$

Haynes, F. E., I 81, 394

Healy, W., I 77, I78, 394

Henry, T. R., 326

Heraclitus, 360

Heredity, I, 2, 4, I9, 63, i I $2 f$.

Hering, E., 22

Hermaphroditism, 47-49

Heron, D., 216, 394

Herrman, C., 394 
Herskovits, M. J., 394

Heys, F., 8I

Hill Folk, 186, 326, 375

Himes, N., 207, 394

Hindus, emigration, 333

Hodson, C. B. S., 385

Hoffmann, H., I38, I39

Hogben, L., 394

Holling worth, L. S., 394

Holmes, O. W., $37 \mathrm{I}$

Holmes, S. J., I47, 170, 195, 214, 236, $272,288,357,385,387,388$, 394,395

Hooke, R., 2

Hookworms, relation to intelligence, 156

Hooton, E. A., 172, 173, 395

Hoover, G., 395

Hormones, 49, $5^{\circ}$

Howard, G. E., I 90

Hume, D., 290

Humm, D. G., I36, I44, 395

Hunt, H. R., 280, 395

Huntington, E., I8I, 19I, 21 I , 21 8, 23I, 236,395

Huntington's chorea, $38,139^{-1} 42$, I $5^{1}$, 257

Hurst, C. C., 395

Huxley, J. S., 39, 50, 83, 85, 395

Huxley, T. H., 5

Ibsen, H. L., 83, 395

Ichthyosis, 105

Idiots, I 27, I 28, I 32, I69, 259, 369

Illegitimacy, $183-186,356,369$

Imbeciles, I27, I28, I30-132, I69, 259,369

Immigrants, marriages of, 194

Immigration, 312-317, 323, 331-337, $339-348,355,384$

Immigration Commission, 316,341

Inbreeding, $349 . f$.

Incas, 349

India, emigration from, 332,333
Infant mortality, $244^{-246}, 250,25 \mathrm{I}$, $261-272$

Infanticide, $310,360,361$

Insane, 369, 370

Insanity, inheritance, 63, I09, I I0, I3I, I36-I $43,250,251$

Intelligence, I 14, I23, I 24, $149 \mathrm{ff}$.

Intelligence quotients, $156,158-167$, $225,233,235,326-328,338$

Isolation, 356

Italy, birth rate, 2I I

\section{J}

Jacob, breeding of cattle, 86

Jaederholm, G. A., I 28

Jamieson, E., 354

Janssens, F. A., 69

Japan, population growth, 297, 307

Japanese, birth rate for, 199,200

emigration of, 332

Jenkins, R. L., 233

Jennings, H. S., 39, 85, 124, 169, 227, 396

Jews, birth rate, 212

Johannsen, W., 95

Johnson, E. R., 370

Johnson, R. H., 195, 368, 385, 396, 400

Jones, D. Caradog, $163,164,233,236$, 396

Jones, D. F., 357, 391

Jones, H. E., 230, 327, 389, 396

Jordan, D. S., 278, 279, 288, 396

Jordan, H. E., 278, 288, 396

Juke family, 183-185, 187, 193, 231 . 375

\section{K}

Kallikak family, 132, 184-187, 193, 231

Kammerer, P., 26, 27, 396

Karyokinesis, 6-8

Keeler, C. E., 122

Keller, X., 234

Keratosis, 105

Key, W. E., 396

Kirkpatrick, C., 344, $34^{8}$. 


\section{IO HUMAN GENETICS AND ITS SOCIAL IMPORT}

Kiser, C. V., 224, 396

Knibbs, G., 297, 318, 396

Knowlton, C., 206, 207

Koch, H. L., I 23

Kuczynski, R. R., 300, 318, $39^{6}$

Kurz, K., 234

\section{$\mathbf{L}$}

Lamarck, J. B., I9, 20

Lamarckism, I9-28, 79, 254

Landman, J. H., 396

Lange, J., I 42, I 79, I 8 I, 396

Lauterbach, C. E., 124, 396

Lawrence, E. M., I61, I63, 170, 397

Leahy, A. M., 164, 170, 397

Leber's atrophy, ${ }_{4} 6$

Legras, A. M., I 80, I 81, 397

Lennes, N. J., 397

Lentz, T., 397

Lenz, F., $110,147,205,216,217,222$, $224,279,388,397$

Lenz, T., 224

Lepidoptera, 44, 47

Lethal factors, $39,5^{8}$

Levasseur, E., 205

Lewis, P. A., 105

Lidbetter, E. J., I 87, 397

Life tables, 240, 24I

Lincoln, A., I 70

Lindsey, A. W., 49, 74, 96, I10, 397

Linkage, $66 f f$.

Little, C. C., 84

Lobster claw, inheritance, IOI, 102

Lombroso, C., I 72-1 74, I 78, 397

London, birth rate for, 216,229

London School of Economics, family allowances, 378

Longevity, 94, II 3, 242, 247, 256, 265, 266

Lorimer, F., 170, 214, 27I, 318, 324, $328,348,387,397$

Lotka, A. J., 303, 391, 397

Ludovici, A. M., 357, 397

Ludwig of Bavaria, 140

Lutz, F. E., 25

Luxenberger, H., I35, I 42

Lycurgus, 360

\section{M}

Macaulay, T. B., I 57

McClung, C. E., 42

McDougall, W., 26, 27, 381, 397

McDowell, E. C., $8 \mathrm{r}$

McKemy, Mexican immigration, 340

Macklin, M., 146

MacPherson, J., 397

Mallet, B., 397

Malpighi, M., 2

Malthus, T. R., 206, 290-294, 318, 398

Malthusianism, 375

Malzberg, B., 397

Manic-depressive insanity, ${ }_{13} 8, I_{4} 2$

Maoris, 286

Marriage, age for, 191 $f f$., 198, 201 rate of, r91 ff., 198-203

Marvin, D. M., I93

Marx, C., 294

Maturation of sex cells, $1_{4}-16$

Mean, 88-9I, 93

Melanesians, 285, 286

Melcher, R. T., $39 \mathrm{I}$

Mendel, G. J., 29, 30, 72, 76

Mendel's law, $29 f f$., 52 ff., 76, 77, 79, $96,98,100,129$, I 30, 349-353

Mental deficiency, I09, I I4, I $26 f f$., I 5 I, 193, 259

Mental Deficiency Committee, I 28, I32, 233

Merriman, W. E., 398

Metropolitan Life Insurance Company, $24 \mathrm{I}$

Mexicans, 317

Mexico, immigration, 31 5, 337-34I

Microcephaly, 127, 128

Migraine, 109

Migration, $330 f f \cdot, 356$

Mill, J. S., I I 2, 365

Miller, H. C., $39^{8}$

Mirabilis, color inheritance, 59

Mirror imaging, I 21-123

Miscegenation, 339, 35 $\mathrm{I}-357$

Mitosis, 6-8, I I, I4

Mjoen, J. A., 352, 357, $39^{8}$

Mode, 88-90 
Mongolian idiocy, 147

Moore, E., 398

Morgan, T. H., 39, 46, 62, 66-69, 73, $74,387,398$

Mormons, birth rate, $21 \mathrm{I}, 230$

Morons, I 27, I 28, I 32, 259, 269

Mortality, $238 \mathrm{ff}$. selective action of, $253 f f$.

Mortality tables, 240, $24 \mathrm{I}$

Mott, F. W., I 37

Mulattoes, 54, 55, 64, 100, 354, 355, 357

Muller, H. J., 84, I 24, 398

Multiple factors, 54, 55, 58, 100, I33I35, I 68, 350

Muncey, E. B., I 39,390

Murchison, C., $174^{-1} 76,398$

Muscular atrophy, inheritance, $I_{4} 6$

Mutations, 78-8I

Mutilations, alleged inherited effects, $24^{-26}$

Myoclonus epilepsy, recessive inheritance, $I_{43}$

Myreson, A., I47, 398

N

Nam family, I 86

Napoleon, 306

Nash, H. B., 394

Natural selection, $253 f$.

Negative eugenics, $366 f f$., 384

Negroes, I, 54, 55, 99, 100, 268, 286, 3II, 3I 2, 3I4, 3I $5,325,326,345$, $346,354,356,357$

Nelson, L., 230

Neo-Malthusianism, 245, 307, 31 I

Nervous diseases, I 46 , I 47

Nettleship, E., I०3

Newman, H. H., I7, 27, 74, 121, 124, I 67, 398

Newsholme, A., 251, 398

New Zealand, birth rate, 199

Nicolai, G. F., 288, $39^{8}$

Nordics, I, I96, 3I3

Norway, birth rate, 2 I I

Notestein, F. W., 192, 222, 223, 398, 401
Nucleus, 5-14

Nurture, I I3 ff., I $49 f f$.

$\mathrm{O}$

Occupation and fertility, 22I-224, 227

and intelligence, $161-165,169$

and mortality, 260-263, 272

Ogburn, W. F., 224, 236, 399

Olkon, D. M., epilepsy in twins, 145

Oogenesis, I4, I 5

Optimum of population, 308-3 10

Orphans, intelligence of, 163,164

Osborn, D., 399

Osborn, F., I70, 214, 271, 318, 324, $328,348,387,397,399$

Otosclerosis, $\mathrm{IO}_{4}$

Otto of Bavaria, inherited insanity, I 40

Owen, R. D., 207, 365

Owens, A. A., 177

\section{$\mathbf{P}$}

Painter, T. S., I3

Pangenesis, 2I, 28

Panmixia, $25^{8}$

Paris, birth rate, 208, 21 3, 229, 324 growth of, 319, 320

Parker, S. L., 324, 395

Parr, Th., alleged longevity, 242

Pascal, B., I 57

Pasteur, L., I05

Pauperism, I83, I 86

Pavlov, I. P., 26

Payne, F., 25

Pea comb in poultry, 59-6I, 64

Pearl, R., 81, 296, 399

Pearl, R. De W., 399

Pearson, K., 87, 91, 94, I I6, II 7, I28, I 53, 193, 207, 214, 254, 255, 266, $270-272,276,277,278,363,399$

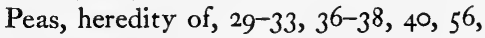
$57,72,77,95$

Pease, M. S., 399

Penrose, E. F., 399

Penrose, L. S., I 34, I 35, 399 


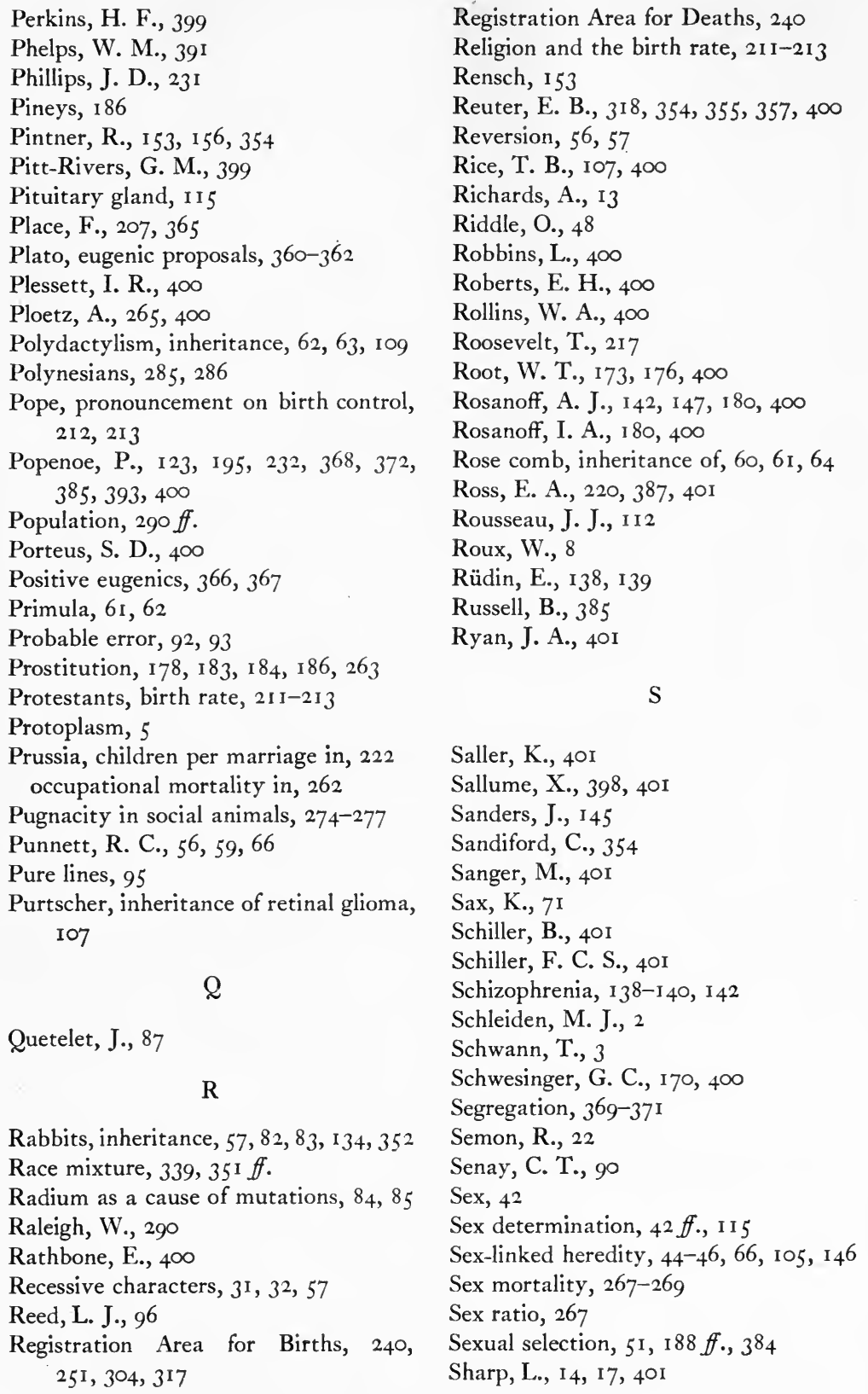


Shaw, G. B., 22

Sherman, M., 326

Shull, A. F., I7, 30, 49, 6I, 64, 40 I

Siamese twins, $122-124,170$

Sinnott, E. W., 39, 49, 64, 40I

Sjögren, T., I30, I31, I 52

Skew curves, 89

Skin color, 54, 55, 100

Skin diseases, inheritance, IO4, IO5

Slavs, 302, $3^{1} 3$

Slye, M., I08

Smillie, W. G., ${ }_{5} 6$

Smith, E. A., 393

Smith, J. C., I 35

Smith, M. R., 218, 40I

Snow, E. C., 266, $40 \mathrm{I}$

Snyder, L. W., 40I

Social problem group, $\mathrm{I} 86$

Sorokin, P., 327, 328, 4 OI

Southey, R., 292

Spartan eugenics, 360

Spearman, C. E., I70, 401

Spencer, H., 3, 202, 278

Spermatogenesis, I 4, I 5

Spermatozoa, 90

Split hands, I00-102

Stabilized rates of increase, 299-304, 3I4, 3I 5

Standard deviation, $9^{\mathrm{I}-93}$

Standardized rates, 238, 239

Stature, 92, 100, II 7

Steggerda, M., 39I

Steinach, E., 49

Sterility, 204

Sterilization, 369, 37I-375

Stevenson, T. H. C., 221, 26I

Still births, 204, 268

Stockard, C. R., 80-82, 401

Stoddard, L., $40 \mathrm{I}$

Stohler, R., I I 8

Stowell, W. L., 259, 389

Strohmayer, W., I 40

Swarthmore college, birth rate of graduates, 217

Sweden, age at marriage, $20 \mathrm{I}$ birth rate, 2 I I

Swezy, O., 9, 392
Sydenstricker, E., 222, 401

Synapsis, I6, I 8, 35, 36, 68

Syndactylism, inheritance, $\mathrm{IOI}-\mathrm{IO} 3$

Syphilis, 103, I26, I36, I37, I 43, 204, 205,263

\section{T}

Tasmanians, 285

Taylor, P., 348, 40I

Taylor, W. R., 16

Telegony, 86

Terman, L. M., I58, I62, I70, 401

Theognis, 359

Thompson, J. A., 27

Thompson, W. S., 214, 244, 245, 25I, $297,317,3$ I $8,328,348,387,402$

Thurstone, L. L., 233

Thyroid gland and mental deficiency, $64, I_{4}, I_{1} 5, I_{2} 6$

Tibbitts, C., 236

Tortoise shell cats, inheritance, 65

Townsend, on population, 290

Translocations, I0, 67, 7 I

Tredgold, F. A., I 26, 402

Tribe of Ishmael, I 86, 232

Tschermak, E. von, 29

Tuberculosis, 48, I05, I 20, I2I, 257

Twins, I 1 7-I 24, I 35 , I 36, I42, I43, I64-I68, I79-I $8 \mathrm{I}$

\section{$\mathrm{U}$}

Ungern-Sternberg, R. von, 203, 402

Unit characters, 52

United States, birth rate, 198-200, 230

marriages in, 202

mortality, 240, 24I, 243, 244 ff., 264

population growth, 297, 298, 303, $3 \circ 4$

Urban areas (see Cities)

Urban migration, 321 ff.

V

Variation, measurement of, $87 f f$.

Variations, classes of, $76 f f$.

production of, $79 . t$. 


\section{I4 HUMAN GENETICS AND ITS SOCIAL IMPORT}

Vassar College, marriage rate of Wiggam, A. E., 366, 385, 402, 403

graduates, 218

Venereal diseases, 263

(See also Gonorrhea; Syphilis)

Vermont, eugenics survey, 232

Verschuer, O. von, I 2O, 402

Vibart, H. H. R., 402

Vienna, birth rate, 208; population, 319

Virchow, R., 3

Virginia statute on sterilization, $37 \mathrm{I}$

\section{W}

Wagner-Manslau, W., 203, 402

Wallace, A. R., 293

Walnut comb, inheritance, 6o, 6I, 64

Walter, H. E., 96, 402

War, biological effects, $274 f f$.

Warthin, A. S., inheritance of cancer, 107

Wassermann tests, I26, I30, I43

Wedgwood, J., I 53

Wiggins, D. M., 225, 389

Willcocks, R. W., I 54

Willcox, W. F., 243, 248, 293, 403

Willoughby, R. R., 403

Wilson, E. B., I7, 43, 403

Wilson, P., II9

Wingfield, A. H., I 24, I65, 403

Winkler, W. F., 234

Winship, A. E., 187, 403

Witschi, E., 49

Wolcott, R., 60

Wolfe, A. B., 308, 403

Woodbury, R. M., 270

Woods, F. A., 23I, $4 \circ 3$

Woods, H. M., 39I

Wright, H., 318, 403

Wright, S., 105

Weismann, A., I4, 22, 23, 27, 258, 402

Wells, G. P., 39, 49, 85

Wells, H. G., 39, 49, 85

Westermarck, H., I90

Whelpton, P. K., 247, 25I, 297, 3I7, 3I $8,324,402$

Whetham, W. C. D., 216

Whetten, N. L., 210,402

Whipple, G. C., 402

White, F. W., 402

Whitney, E. A., I 28, I8I

Whitney, L. F., I27, 19I, 21 I, 231, 234, $236,395,402$

Wiehl, 243

Wieman, H. L., 6, 7, I5

\section{$\mathrm{X}$}

$X$-chromosomes, 13, 42 ff., 66, 69, 73

$X$-rays as a cause of mutations, 78,79 , 84,85

\section{Y}

$Y$-chromosomes, 42, 43, 47, 51, 69, 73

Yale graduates, birth rate, 217,231

Yerkes, R. M., 403

Young, T. E., 262, 403

\section{Z}

Zeleny, C., 90

Zeleny, L. D., I 74, I75

Zero family, I 86

Zimmerman, C. C., 327, 328, 4 OI 


$$
\text { - }
$$





995 
